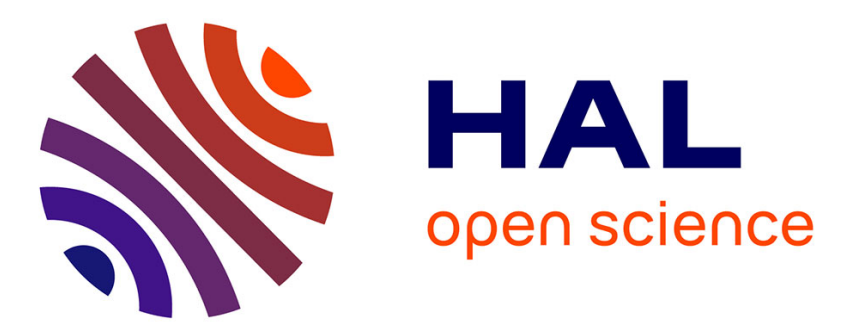

\title{
Alternative Technologies That Facilitate Access to Discrete Metal Complexes
}

Audrey Beillard, Xavier Bantreil, Thomas-Xavier Métro, Jean Martinez, Frédéric Lamaty

\section{- To cite this version:}

Audrey Beillard, Xavier Bantreil, Thomas-Xavier Métro, Jean Martinez, Frédéric Lamaty. Alternative Technologies That Facilitate Access to Discrete Metal Complexes. Chemical Reviews, 2019, 119 (12), pp.7529-7609. 10.1021/acs.chemrev.8b00479 . hal-02325837

\section{HAL Id: hal-02325837 \\ https://hal.science/hal-02325837}

Submitted on 16 Mar 2021

HAL is a multi-disciplinary open access archive for the deposit and dissemination of scientific research documents, whether they are published or not. The documents may come from teaching and research institutions in France or abroad, or from public or private research centers.
L'archive ouverte pluridisciplinaire HAL, est destinée au dépôt et à la diffusion de documents scientifiques de niveau recherche, publiés ou non, émanant des établissements d'enseignement et de recherche français ou étrangers, des laboratoires publics ou privés. 


\title{
Alternative Technologies That Facilitate Access to Discrete Metal Complexes
}

\author{
Audrey Beillard, Xavier Bantreil,*(i) Thomas-Xavier Métro,*(i) Jean Martinez, ${ }^{(0)}$ and Frédéric Lamaty*(i) \\ Institut des Biomolécules Max Mousseron (IBMM), UMR 5247, CNRS, Université de Montpellier, ENSCM, Campus Triolet, Place \\ Eugène Bataillon, 34095 Montpellier cedex 5, France
}

ABSTRACT: Organometallic complexes: these two words jump to the mind of the chemist and are directly associated with their utility in catalysis or as a pharmaceutical. Nevertheless, to be able to use them, it is necessary to synthesize them, and it is not always a small matter. Typically, synthesis is via solution chemistry, using a roundbottom flask and a magnetic or mechanical stirrer. This review takes stock of alternative technologies currently available in laboratories that facilitate the synthesis of such complexes. We highlight five such technologies: mechanochemistry, also known as solvent-free chemistry, uses a mortar and pestle or a ball mill; microwave activation can drastically reduce reaction times; ultrasonic activation promotes chemical reactions because of cavitation phenomena; photochemistry, which uses light radiation to initiate reactions; and continuous flow chemistry, which is increasingly used to simplify scaleup. While facilitating the synthesis of organometallic compounds, these enabling technologies also allow access to compounds that cannot be obtained in any other way. This shows how the paradigm is changing and evolving toward new technologies,

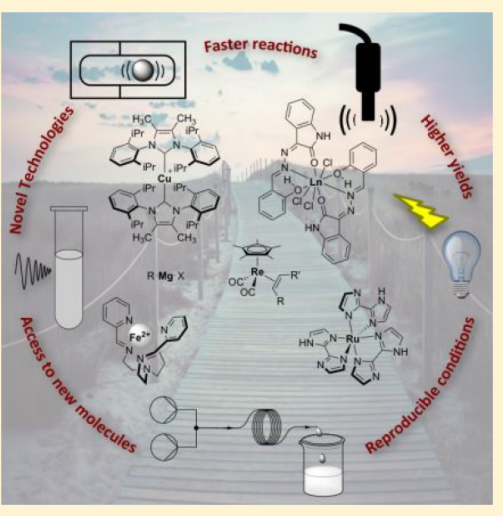
without necessarily abandoning the round-bottom flask. A bright future is ahead of the organometallic chemist, thanks to these novel technologies.

\section{CONTENTS}

1. Introduction

2. Technologies

2.1. Grinding and Milling

2.2. Microwave Irradiation

2.3. Ultrasound Activation

2.4. Photochemistry

2.5. Continuous Flow

3. Contribution of Each Technology Compared to Classical Syntheses

4. Lithium and Magnesium

4.1. Mechanochemical Access to Grignard Reagents

4.2. Microwave Irradiation for the Synthesis of Magnesium Complexes

4.3. Ultrasonic Procedures

4.4. Continuous Flow Syntheses

5. Groups 2, 3, 4, and 5

5.1. Mechanochemical Procedures for Complexes of Groups 2, 3, and 4

5.2. Microwave Irradiation for the Synthesis of Vanadium Complexes

5.3. Synthesis of Vanadium Complexes Using Ultrasound

6. Group 6

6.1. Mechanosynthesis of Molybdenum and Chromium Complexes

6.2. Microwave Irradiation for the Synthesis of Group 6 Metal Complexes
7530

7530

7530

7531

7531

7532

7532

7533

7534

7534

7534

7535

7535

7537

7537

7537

7538

7538

7538

7539
6.3. Photochemistry for Synthesis of Group 6 Metal Complexes

6.4. Continuous Flow Chemistry for Synthesis of Chromium Complexes

7. Group 7

7.1. Mechanosynthesis of Rhenium Complexes

7.2. Microwave Irradiation for the Synthesis of Group 7 Metal Complexes

7.3. Photochemical Synthesis of Manganese and Rhenium Complexes

8. Group 8

7543

7545

7545

7545

7546

7549

7550

8.1. Synthesis of Iron and Ruthenium Complexes Using Ball Milling

7550

$\begin{array}{ll}\text { 8.2. Microwave Procedures } & 7552 \\ \text { 8.3. Ultrasonic Irradiation for Iron Complexes } & 7562\end{array}$

8.4. Syntheses of Iron and Ruthenium Complexes Using Photochemistry

8.5. Continuous Flow Conditions to Prepare Iron and Ruthenium Complexes

7563

9. Group 9

9.1. Synthesis of Cobalt and Rhodium Complexes by Mechanochemistry

9.2. Procedures Using Microwave Irradiation

7566

7566

7566

7569

9.3. Photochemical Synthesis of Iridium Complexes

7573

10. Group 10

7574

10.1. Mechanochemical Approach 
10.2. Microwave Irradiation

10.3. Ultrasounds for the Synthesis of a Nickel Complex

10.4. Continuous Flow Synthesis of Nickel Complexes

11. Group 11

11.1. Mechanosynthesis and Coinage Metals

11.2. Microwave Synthesis of Group 11 Metal Complexes

11.3. Ultrasounds for the Synthesis of Copper and Silver Complexes

11.4. Continuous Flow for the Synthesis of NHC Copper Complexes

12. Group 12

12.1. Mechanosynthesis of Group 12 Metal Complexes

12.2. Microwave Irradiation for the Synthesis of Group 12 Metal Complexes

12.3. Ultrasonic Activation and Mercury

13. Mechanochemical Access to Aluminum and Indium Complexes

14. Group 14

14.1. Mechanosynthesized Lead Complexes

14.2. Microwave Syntheses of Tin and Germanium Compounds

14.3. Ultrasonic Bath for the Synthesis of Lead Complexes

15. Group 15: Syntheses by Ball Milling

16. Lanthanide

16.1. Mechanochemical Approach

16.2. Microwave Syntheses

17. Conclusion

Author Information

Corresponding Authors

ORCID

Notes

Biographies

Acknowledgments

List of Abbreviations

References

\section{INTRODUCTION}

Over the past decade, remarkable developments of new synthetic procedures for the production of discrete metal complexes have been described. Microwave-assisted, ${ }^{1}$ electrochemical, ${ }^{2}$ photochemical, ${ }^{3}$ mechanochemical, ${ }^{4}$ and continuous flow procedures ${ }^{5}$ have increased yields, while reducing reaction times and environmental impact. In addition, many procedures are highly selective and lead to the desired product with minimal byproducts.

The impressive number of discrete metal complex applications can explain such an increase in the number of innovative strategies to produce them. Indeed, metal complexes are frequently used for their catalytic properties such as in cross-coupling reactions, olefin metathesis, or even oxidation and reduction reactions. Various metal complexes also exhibit biological properties such as antifungal, antibacterial, antimalarial, or anticancer activities. ${ }^{6-17}$ Due to their luminescent properties, they are also widely used as taskspecific materials.

The number of their synthetic procedures has also increased using classical solution synthesis, but many of these procedures have numerous disadvantages such as long reaction times and, in many cases, low to moderate yields. In addition, these procedures may have a negative environmental impact. Consequently, these last years, new strategies were deployed to synthesize discrete metal complexes more efficiently.

This review focuses on the use of enabling technologies for the syntheses of discrete metal complexes. The synthesis of coordination complexes, which would include coordination polymers, metal-organic frameworks, etc., will not be considered herein. Thus, syntheses of discrete metal complexes utilizing mechanical forces, microwave irradiation, ultrasound activation, photochemistry, and continuous flow will be summarized and discussed below. The syntheses of metal complexes using established electrochemical procedures are deliberately excluded in this review, which focuses on newer technologies. Following an introductory part describing the various technologies available for activation, the review is organized according to the periodic table, by metallic element group.

\section{TECHNOLOGIES}

All of these technologies have been known and used for some time. The first use of a mortar and pestle in chemistry was dated to the fourth century $\mathrm{BC}$ and described the grinding of cinnabar and acetic acid. Photochemistry is used by plants for photosynthesis. Ultrasonic waves are produced by different animals such as bats to detect their prey, while the most wellknown use of microwaves is cooking. Unfortunately, the application of these technologies to organic synthesis was neglected until the 1980s but then was considerably developed. In this introductory part, each new technology will be discussed in detail.

\subsection{Grinding and Milling}

Mechanochemistry refers to reactions "induced by the input of mechanical energy". 4,18 There are different modes of mechanical activation, and this review will focus on activation by grinding and milling. Mechanical energy is usually produced by shock or friction between two different surfaces. For a long time, this approach was neglected, and solution-based syntheses were developed as default. However, recently, considerable efforts were made to develop new procedures, and this technique has emerged due to the numerous advantages it provides. Generally, reactions are performed in the solid state by grinding. Using a mortar and pestle is the oldest technique to induce reactions by mechanical forces. The historical mechanochemical reaction realized by Faraday was performed using the readily available mortar and pestle. For reactions requiring a long grinding time, the affordable mortar and pestle can be replaced by a ball mill, which also provides more reproducible results. Various apparatuses, mostly vibratory and planetary ball mills, were developed to perform chemical syntheses by mechanical means. This equipment is classically used to reduce the particle size of reagents and to homogenize the reaction mixture. Trivially, reagents and one or several balls are added into a jar which is closed and subjected to grinding for a specific time at a preset frequency. Sizes and materials of jars and balls can be changed, among Teflon, PMMA (poly(methyl methacrylate)), stainless steel, zirconium oxide, agate, and tungsten carbide, depending on the application. An important feature of using ball milling is the possibility of performing reactions under solvent-free or solvent-less conditions. ${ }^{19}$ The combination of a highly concentrated 
reaction mixture with excellent mass transfer dramatically increases the reaction rate compared to classical solution chemistry. In addition, ball milling as a solvent-free methodology gives access to compounds that cannot be obtained in solution, even in drastic conditions. ${ }^{20}$ Nevertheless, users should be aware that contamination resulting from abrasion of the grinding jar/balls ( $<30$ to $40000 \mathrm{ppm}$ ) can be found in the products, but this can be reduced by a judicious choice of the milling material. $^{21,22}$ This contamination from wear could be a problem depending on the applications of the synthesized products (e.g., pharmaceutical products).

For scale-up, other equipment has been developed. ${ }^{23,24}$ Eccentric ball mills, also called eccentric vibratory ball mills, ${ }^{25}$ are composed of a vibrating unit attached to a grinding chamber displaced from its center of gravity. This arrangement features a 5-300 L grinding vessel, which can lead to the formation of kilograms of products.

Another approach to scale-up is the reactive extrusion process. Typically, reactants are introduced in a barrel containing one (or two) screws that mix and carry the material from one end to the other, where products are recovered or can be recycled into the feeding port of the barrel. The main advantage of this technique is the possibility to work in a continuous manner. ${ }^{26}$ Typically, one (or two) screws are used to mix reagents and carry the material to the end of the process, where products are recovered or can be reinjected into the cavity. This method can be used to make grams or kilograms of products. Both pieces of these scale-up equipment can be heated at elevated temperatures.

\subsection{Microwave Irradiation}

The use of microwaves in chemical synthesis has been known since the 1980s, with the first syntheses performed in domestic microwave ovens. ${ }^{27}$ Since then, many developments have been made. ${ }^{28-31}$ Some of these ovens were modified to improve their use in chemistry; however, because temperature and pressure were not controlled, they were often dangerous (potential explosion at high temperature and pressure), and reproducibility was difficult. Nowadays, dedicated instruments have been developed and are used in chemistry laboratories with adequate safety, making microwave activation a common tool used in synthesis. One should emphasize that currently, because of safety reasons, domestic ovens should not be used to perform any chemistry. Nevertheless, it is important to note that some of the pioneering work in organometallics synthesis reported in this review was carried out in such ovens.

Microwave technology makes use of an electromagnetic wave generated by a magnetron at a frequency which ranges between 0.3 and $300 \mathrm{GHz}$, very often factory set to $2.45 \mathrm{GHz}$ to avoid interference with other instruments using microwaves. Under microwave irradiation, polarized molecules in a reaction mixture will constantly try to realign their dipole moment with the oscillating electric field, thus creating molecular motion leading to intermolecular friction and collisions and ultimately generating heat (dipolar polarization) in the core of the irradiated mixture. The second important heating mechanism, known as ionic conduction, involves charged compounds (usually ions). When submitted to an oscillating electric field, charged compounds will adopt oscillating trajectories in the solution and create many intermolecular collisions of which the energy dissipates as heat. The reaction mixture can reach very high temperatures, reaching a maximum set between 250 and $300{ }^{\circ} \mathrm{C}$ for commercial instruments. According to the
Arrhenius equation, this efficient increase of temperature will translate to faster reactions. ${ }^{32}$ Very often instruments dedicated to synthesis make use of sealed tubes that allow pressure buildup in the course of the reaction. ${ }^{33}$ Generally, one important parameter is the choice of the solvent and more specifically its capacity to absorb microwaves and generate heat. Nevertheless, in this review dedicated to organometallic chemistry and in all the cases described, the presence of a metal or a metallic salt ensures the absorbance of the microwaves, thus enabling the transformation to occur. ${ }^{34}$ Moreover, this absorption and concomitant temperature increase on very localized areas of the mixture may efficiently promote a reaction (hot spot theory).

\subsection{Ultrasound Activation}

The sound spectrum is divided into four intervals: infrasound with frequencies ranging from 0 to $16 \mathrm{~Hz}$, audible sound between $16 \mathrm{~Hz}$ and $16 \mathrm{kHz}$, ultrasound $16 \mathrm{kHz}$ to $200 \mathrm{kHz}$, and finally hypersound with frequencies higher than $100 \mathrm{GHz}$. This part will describe the excitation of molecules by ultrasound. Ultrasounds are elastic waves having the properties of sound waves, namely, the propagation of mechanical disturbances in an elastic media. The main characteristic of these waves is their frequency, which depends on the propagation speed of the sound and the wavelength associated with this wave. Ultrasound waves in a liquid produce a pressure variation responsible for the creation, growth, oscillation, and finally the implosion of a gas bubble. This phenomenon is known as cavitation. ${ }^{35}$ The implosion of gas bubbles is accompanied by mechanical and chemical effects that will be transformed into energy. ${ }^{36,37}$ Sonochemistry is based on the acoustic energy and the cavitation phenomenon produced by ultrasound. ${ }^{38}$ It should also be noted that upon ultrasonic activation radicals may be formed in the reaction mixture. A transducer is used to produce an ultrasound, which relies on the properties of piezoelectric materials to convert electric energy into acoustic energy.

From a practical point of view, ${ }^{39,40}$ the first and most frequently used equipment for sonochemistry is the ultrasonic bath. These baths allow working with frequencies ranging from 20 to $60 \mathrm{kHz}$ and low sound power under indirect irradiation. A vibratory source is fixed at the bottom of the tank to irradiate the liquid (often water) which transfers energy to the reaction. Ultrasound is inhomogeneously distributed into the reactor, and its intensity is highly dependent on the position of the reaction mixture inside the tank. Since low reproducibility was obtained for reactions, these baths are nowadays mostly used to dissolve compounds, clean surfaces, break emulsions, or degas liquids. "Cup-horn" reactors permit a direct and homogeneous irradiation, which is 50 times more powerful than classical ultrasonic baths. Ultrasonic probes were also developed for a better transfer of energy to the sample. The device is composed of a generator that converts the electric current into high frequency electrical energy, which is then converted into mechanical energy using an ultrasonic converter, an amplificatory probe to increase the amplitude of the wave, and finally an ultrasonic probe to transfer the energy to the sample. With ultrasonic probes, the irradiation is direct, and the acoustic power is more than a hundred times more powerful than ultrasonic baths. In addition, this equipment is resistant to hot spots, erosion, and mechanical stress. To perform highly energetic reactions, high frequency reactors were developed, with a direct irradiation from the 
bottom to the top of the reactor using piezoelectric ceramics attached to the bottom of double-jacketed reactors. Nevertheless, these reactors quickly deteriorate due to the powerful temperature elevation resulting from the ultrasound.

Different parameters can affect the cavitation phenomenon. The frequency of ultrasound determines the lifespan of the bubbles: a higher frequency makes the bubbles grow and collapse faster. Temperature and hydrostatic pressure are also important. Increasing pressure or temperature will lead to poorer cavitation, but the energy or the diffusion is increased, respectively. Although low temperatures promote better cavitation, experiments demonstrate that better yields are achieved with elevated temperatures. The medium is an important parameter. Solvents with a high vapor pressure (most organic solvents) decrease the intensity of the cavitation, but the presence of dissolved gas improves the nucleation step of the bubbles. ${ }^{41}$

Even though enhancing reactions with ultrasonic activation is very promising, the reproducibility of the reactions, because of the difficulty in controlling different physicochemical parameters, is a huge limitation of sonochemistry. Equipment and experimental conditions have to be precisely reported to allow the replication of the experiments. In addition, the scaleup of sonochemical reactions to date remains difficult.

\subsection{Photochemistry}

Interactions between light and matter have long been known and are used in nature, e.g., photosynthesis, the phenomenon of eyesight, and tanning. ${ }^{42}$ Upon light exposure, the energy of the photon absorbed by a molecule or material will allow an electron to transfer from its fundamental state to an excited state of higher energy. ${ }^{43-45}$ The activated molecule can directly react or transfer its energy to another reactant. In the latter case, the molecule is called a photosensitizer. In principle, photochemistry can be performed in the gas phase, solid state, or solution, although photochemical reactions in solution are the most developed. ${ }^{46}$

The most consistent and sustainable source of light is sunlight. Photochemistry performed with this source would be the ideal situation. Unfortunately, most reagents absorb in the UV (ultraviolet) region (between 200 and $300 \mathrm{~nm}$ ), and the Earth's atmosphere mostly filters ultraviolet rays. Therefore, mercury-vapor lamps, namely, gas discharge lamps that use an electric arc through vaporized mercury to produce light, were developed to work in a range between 250 and $600 \mathrm{~nm} .^{47}$ Lamps with a low pressure (5-10 atm) were first developed, but then medium- or high-pressure lamps (5-100 atm) were used to improve the efficiency. Unfortunately, mercury lamps are highly toxic in the case of breakage and have a short lifespan. An efficient, albeit still expensive, alternative is the UV LED lamp (ultraviolet light-emitting diodes) which allows one to work at high power and also possess a longer lifetime. Other lamps can be used to work at a specific wavelength such as sodium lamps, which irradiate at $600 \mathrm{~nm}$. Interestingly, additional solid or liquid filters may be used to restrict the irradiation wavelengths. With regard to the reactors, they have to be transparent to the desired working wavelengths. Most reactors used for photochemistry are made of quartz, which absorbs only at $254 \mathrm{~nm}$. Pyrex reactors absorb under $275 \mathrm{~nm}$, while standard window glass absorbs at wavelengths lower than $350 \mathrm{~nm}$.

When performing photochemistry, the first parameter to consider is the irradiation wavelength to determine which reactor and light source to use. Recording the UV spectra of all photoactive species may help to find the maximum of absorption of each molecule and determine the best compromise. Of note, the absorption energy is highly dependent on the choice of the irradiation wavelength. The choice of solvent is also important as solvent can absorb the light and react under specific conditions. Chlorinated solvents are often avoided due to the possibility of $\mathrm{C}-\mathrm{Cl}$ bond cleavage, leading to chlorination reactions. Numerous solvents also inhibit photochemical reactions because of high UV absorption, such as cyclohexane at $215 \mathrm{~nm}$ or hydrocarbon solvents around $330 \mathrm{~nm}$. The purity of the solvent should also be considered as impurities can absorb and lead to low conversions. Interestingly, the choice of solvent may lead to different conversions or different final products. To conclude, three characteristics have to be considered for the choice of solvent. It should be transparent at the selected irradiation wavelength, free of impurities, and it should dissolve reactants and reagents.

In addition to the chemical yield, the quantum yield is also defined in photochemistry to evaluate the efficiency of a photochemical transformation. This yield corresponds to the number of events (photochemical-induced transformations) divided by the number of absorbed photons. Typically, the quantum yield should be equal to 1 when one molecule absorbs one photon. Since not all photons are absorbed productively, it is usually lower than 1 . However, for chain reactions, quantum yields can be higher than 10000 . Nevertheless, in the case of low quantum yield, the chemical yield is not correlated and can be excellent.

\subsection{Continuous Flow}

Continuous flow chemistry is one of the major advances in the last few decades in terms of new technology for synthesis. ${ }^{5,48}$ Although chemists are still attached to conventional batch chemistry using round-bottom flasks and stirrers to homogenize reactant solutions, the transition to continuous flow chemistry is taking place. The benefits of working in a continuous flow are numerous. In fact, compared with conventional chemistry, working in microfluidic mode enables one to improve heat and mass transfers and consequently the kinetics of a reaction. The possibility of performing reactions under pressure also allows one to work in superheated solvent (above the boiling point), similar to sealed microwave reactors. This represents one of the many reasons why microwave reactions are generally translatable to continuous flow. ${ }^{49}$ In addition, because each part of the tubing only contains small amounts of reagents in contact with each other, the risks associated with possible reaction exothermicity are limited. This similarly applies to the use of explosive reagents. ${ }^{50}$ In terms of reactivity, it is also possible to control the stoichiometry of reagents locally because only a few molecules are mixed at any one time and thus influence the selectivity of a reaction. ${ }^{51}$ In addition, the use or in situ preparation of highly reactive species is very convenient in continuous flow. However, one of the main limitations of continuous flow chemistry is the use or the formation of solids that lead to reactor clogging.

Different suppliers offer turnkey equipment including pumping systems, via HPLC or peristaltic pumps, variable and interchangeable reaction modules with integrated heating, such as reactor coils or microfluidic chips, and the possibility of adding columns that may contain purification resins or 
Table 1. Chart Comparing Some Enabling Technologies and Classical Solution Chemistry for the Synthesis of Organometallic Complexes

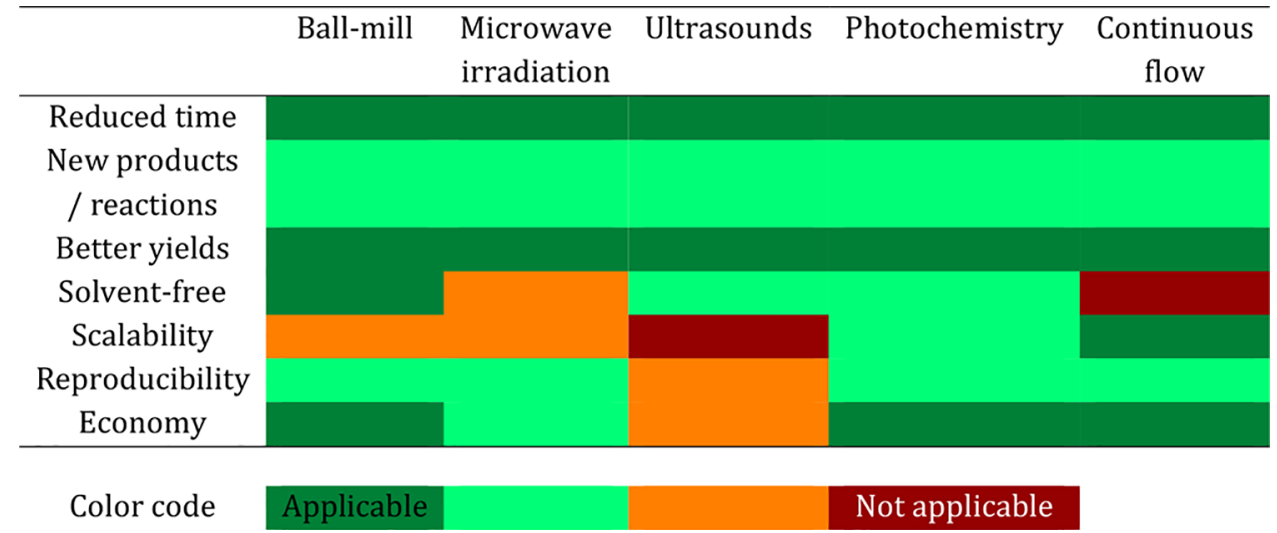

heterogeneous reagents. The assembly can be improved by combining it with an automated reagent sampler and a fraction collector, the whole being controlled by a computer. It is also possible to perform low-temperature reactions using the appropriate add-on module. To further improve the potential of such equipment, real-time and in situ analysis of the reaction mixture through IR (infrared) and UV spectroscopy, as well as gas chromatography, can be used to monitor the reaction. With this in mind, the Poliakoff group recently developed a selfoptimizing algorithm for the reaction of aniline with dimethyl carbonate in supercritical $\mathrm{CO}_{2}{ }^{52}$

Flow chemistry is very adaptable. Recently, the development of photoredox catalysis resulted in suppliers developing photochemical reactors with different wavelengths depending on the targeted application. ${ }^{53}$ Similarly, the use of gases is not a limitation in continuous flow due to the development of tubein-tube reactors, which feature an inner gas permeable membrane. ${ }^{54}$

If the turnkey equipment is relatively expensive or not necessarily adapted to certain types of chemistry, it is possible to build homemade continuous flow systems from spare parts. ${ }^{55}$ This approach also has the advantage of modularity compared to commercially available equipment. It is also possible to monitor a continuous flow reaction while remaining in a distant office, thus conducting continuous flow experiments via remote control and cloud chemistry. ${ }^{56}$

\section{CONTRIBUTION OF EACH TECHNOLOGY COMPARED TO CLASSICAL SYNTHESES}

A summary of the benefits provided by the use of the enabling technologies selected in this review over solution chemistry for the preparation of discrete metal complexes is presented in Table 1. Even though they share many benefits, some particular advantages can be highlighted.

First, one general advantage is the reduction of reaction time. Many reports compare microwave activation or mechanochemistry to classical syntheses, and in most cases, the use of these techniques allows one to reduce the reaction time from several hours or days to a few minutes. The use of ultrasound or continuous flow techniques gives similar enhancements, but only a few reports show a direct comparison of these techniques with conventional conditions, especially for the synthesis of organometallic complexes. In the case of photochemistry, the comparison is less appropriate since few photochemical reactions would proceed in conventional conditions.

This last point is of high interest. Indeed, the enabling technologies described above make it possible to carry out reactions that would not be successful by conventional methods. This is particularly the case for photochemistry which is often used in organic chemistry to achieve transformations that could not occur without this activation. This aspect is also currently under development for the preparation of organometallics. More generally, the use of microwave activation in closed-vessel mode allows the reaction of poorly reactive substrates which would normally remain inactive under refluxing conditions. In mechanochemistry, the number of examples dealing with the formation of new molecules, which cannot be synthesized in solution, or with a change in selectivity compared to solution is growing. ${ }^{20}$ In the case of ultrasound waves, activation modes may differ, but the phenomenon of cavitation or the generation of transient species such as radicals would promote difficult reactivities. To date, for continuous flow, it is difficult to evaluate the feasibility of new reactions or access to new organometallic complexes since reports dealing with the synthesis of discrete metal complexes are scarce. However, since this technology is increasingly being developed, and more often than not combined with other activation techniques, it will most probably demonstrate its effectiveness in this synthetic area in the near future.

The most important benefit of mechanochemistry using ball milling compared to other conditions is the possibility of working under solvent-free or solvent-reduced conditions. Initial developments of mechanochemistry consisted of grinding mixtures of solids to synthesize alloys or inorganic compounds. Subsequently, ball mills were used to grind solidliquid or even liquid-liquid reaction mixtures. Due to the absence of solvent, reactants are present in high concentration and have excellent mass transfer due to milling. Reaction times are decreased, and products are generally obtained in higher yield and purity. In terms of waste reduction, mechanochemistry has proven its efficiency. Green metrics have demonstrated the reduced environmental impact of synthesis performed in a ball mill compared to classical conditions. This is because of solvent-free conditions, increased yields, reduced reaction times, and simplified purification.

The major advantage of microwave irradiation, as provided by specially adapted equipment, is to reach and maintain high 
Scheme 1. Mechanochemical Generation of Grignard Reagents

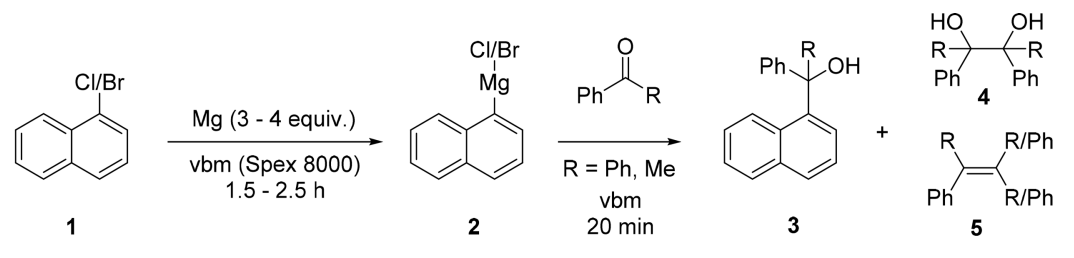

temperature in a reaction vessel, most commonly in a sealed tube, and hence accelerate the reaction. Furthermore, most salts and organometallic species present in a reaction mixture are excellent microwave absorbers, which contribute to the efficiency of the process. For the preparation of organometallic complexes, the reaction mixture is often ground in a mortar and pestle before irradiation. Authors have also described solvent-free syntheses using an additive (such as aluminum) to enhance microwave absorption. ${ }^{57}$ In a similar way, but with different types of activation, sonochemistry, by generating intense local effects, may promote impossible or slow transformations.

While providing a way of promoting specific transformations, photochemistry has the advantage of being inexpensive and producing little waste. The ultimate aim is to use the sun as the light source. However, since the reproducibility of such reactions would depend on the weather, most processes are performed using light bulbs or LEDs (lightemitting diodes) with a controlled power source.

Using these techniques, yield and selectivity can be increased, with the resulting formation of fewer byproducts. Increased yield and selectivity also imply a simplified workup of the reaction mixture, with sometimes a simple filtration or extraction to give the pure product, thus avoiding purification using chromatographic techniques. This is very advantageous, particularly when it comes to scaling-up reactions.

Furthermore, combinations of these technologies are rapidly developing, especially in the case of flow chemistry where the equipment setup can include modules providing alternative activations, such as microwaves, ultrasound, or photochemistry.

\section{LITHIUM AND MAGNESIUM}

In contrast to elements of other groups, organometallic species involving lithium and magnesium are generally unstable and highly reactive. They thus have to be reacted rapidly with an electrophilic species. New procedures have been developed in the literature to form them in situ using enabling technologies such as milling, microwave irradiation, ultrasound, and continuous flow. These innovations will be described briefly below.

The Grignard reaction is one of the most common synthetic pathways in organic chemistry. ${ }^{58}$ Reaction reproducibility can be affected by different factors: metal purity, degree of association in solution, or Schlenk equilibrium. Generally, the reaction needs to be activated. ${ }^{59}$ On the other hand, lithiation can facilitate reactions that are not possible using Grignard reagents; however, lithiated species are extremely sensitive to air and can be harmful. A range of methods of preparation and reactions of these species have been developed (chemically, mechanically, thermally, or sonochemically) and will be discussed below.

\subsection{Mechanochemical Access to Grignard Reagents}

In 2001, Harrowfield et al. investigated the activation of magnesium in a ball mill to generate Grignard reagents from naphthalene halides (bromide and chloride). ${ }^{60}$ The reaction of magnesium (50 mesh) in excess (up to 4 equiv) with 1-chloroand 1-bromo-naphthalene $\mathbf{1}$ was performed in a Spex 8000 vibratory ball mill (Scheme 1). The reactor was filled and closed inside a glovebox to ensure an inert atmosphere during the reaction. After 1.5 to $2.5 \mathrm{~h}$ of reaction, it was possible to recover the corresponding Grignard reagent 2 , which could be stored inside the glovebox without decomposition for up to 10 weeks. Subsequent reaction with acetophenone or benzophenone provided the expected tertiary alcohols 3-5. Excess of magnesium in the milling jar, however, promoted the formation of side products resulting from a McMurry reaction. 4.2. Microwave Irradiation for the Synthesis of Magnesium Complexes

The Hulshof group described the synthesis of highly reactive Grignard reagents from magnesium turnings and a halide substrate under microwave irradiation. The species generated in situ were trapped with $\mathrm{CO}_{2}, \mathrm{CS}_{2}$, or benzaldehyde to form corresponding products $\mathbf{6 - 8}$ in $30-88 \%$ yield (Scheme 2$){ }^{61}$

Scheme 2. Microwave-Assisted Synthesis of Grignard Reagents

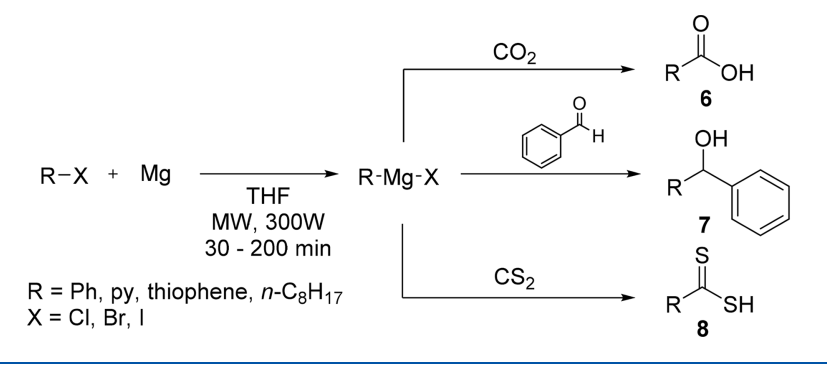

In comparison with conventional methods, reaction times and yields were similar, but microwave-induced discharge on the magnesium surface led to a considerably shorter initiation time and eliminated the use of initiators such as iodide or dibromoethane, which are toxic. In addition, the method limited the formation of byproducts. Inspired by this work, the Kappe group demonstrated that the reaction was highly dependent on the electromagnetic field of the microwave irradiation and not on the temperature. ${ }^{34}$ Microwave irradiation using low-field density conditions led to an apparent acceleration of the insertion of $\mathrm{Mg}$ metal into the carbon-halide bond and reduced the initiation period. Conversly, using high-field density conditions resulted in the creation of carbonaceous material by solvent decomposition, which covered the $\mathrm{Mg}$ metal and prevented access to the organohalogen reagent, thus stopping the formation of the organomagnesium complex. 


\subsection{Ultrasonic Procedures}

Cravotto et al. described the activation of a magnesium surface under ultrasonic irradiation. ${ }^{59}$ The procedure was found to be safe, efficient, and reproducible. A $300 \mathrm{kHz}$ cup horn was found to be the most efficient ultrasonic device. All Grignard reagents 10 were prepared in quantitative yield after less than 1 $\mathrm{h}$ of reaction and then reacted with a sterically hindered ketone, benzophenone, to furnish corresponding tertiary alcohols 11 in $84-94 \%$ yields (Scheme 3). This ultrasonic

Scheme 3. Ultrasound Synthesis of Grignard Reagent

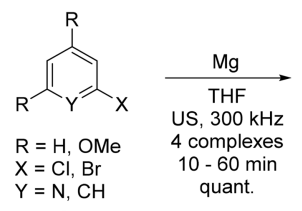

9

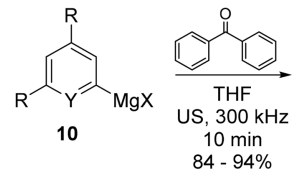

$84-94 \%$

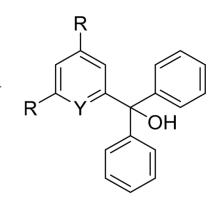

11 procedure was applied successfully to moderately reactive aryl halides such as 2-chloropyridine and 3,5-dimethoxy-bromobenzene. In comparison to the MW procedure, the US procedure enabled working under milder and safer conditions and avoided the risk of arcing.

\subsection{Continuous Flow Syntheses}

Using continuous flow chemistry, Grignard reagents could be generated in situ and reacted with electrophile to prepare various kinds of products: $\mathrm{N}, \mathrm{N}$-diethyl-4-(3-fluorophenylpiperidin-4-ylidenemethyl)benzamide $\mathbf{1 3}$ as a $\delta$-opioid receptor agonist, $^{62}$ 3-hydroxymethylindoles $15{ }^{63}$ 2,4,5-trifluorobenzoic acid $17,{ }^{64}$ diaryl-substituted methyl alcohol $19,{ }^{65}$ and propargylic alcohols and analogues $\mathbf{2 0 - 2 2 ^ { 6 6 }}$ (Scheme 4). In contrast to microwave or ultrasound syntheses, Grignard species were generated by the reaction of a halide compound with $\mathrm{RMgCl}$, which had to be less stable than the desired Grignard reagent. The formation of the Grignard reagent could be monitored in-line by $\mathrm{IR}^{65}$ In addition, a continuous flow

Scheme 4. In Situ Generation of Grignard Reagents for the Formation of Organic Compounds in Continuous Flow

Synthesis of a $\delta$-opioid receptor agonist ${ }^{62}$
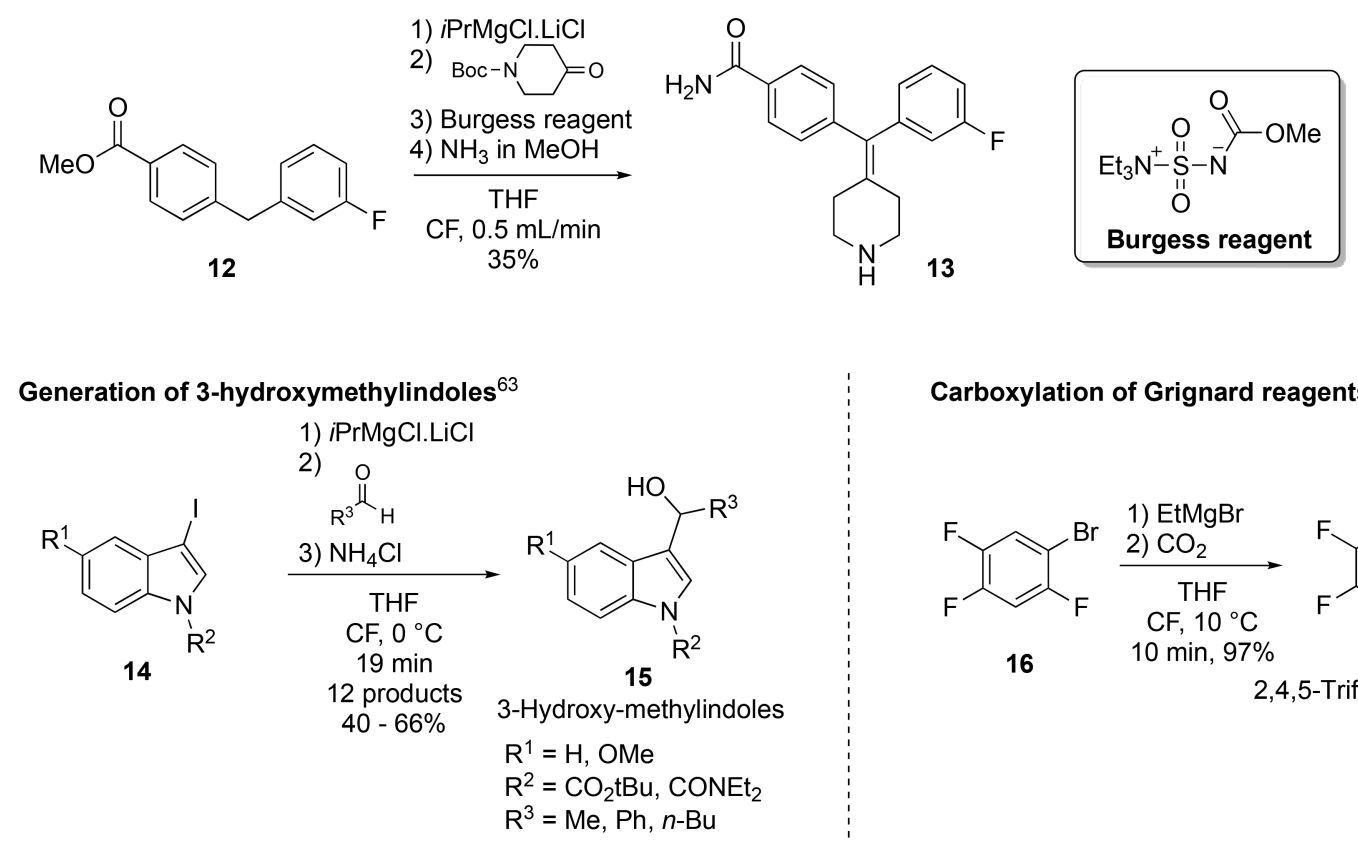

Carboxylation of Grignard reagents ${ }^{64}$

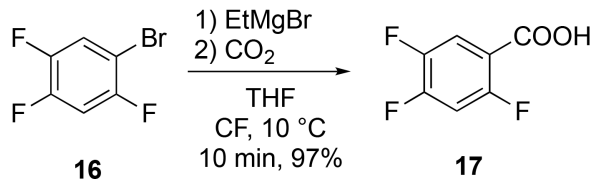

2,4,5-Trifluorobenzoic acid

Continuous preparation of diarylmethyl alcohols ${ }^{65}$

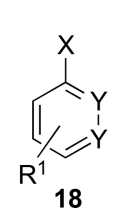

$\mathrm{X}=\mathrm{Br}$, I

$\mathrm{Y}=\mathrm{CH}, \mathrm{N}$

$\mathrm{R}^{1}=\mathrm{CH}_{3}, \mathrm{CF}_{3}, \mathrm{Cl}$

$\mathrm{CN}, \mathrm{OMe}, \mathrm{CO}_{2} \mathrm{Et}$

\section{1) $\mathrm{PrMgCl} . \mathrm{LiCl}$}

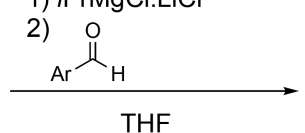

THF

$\mathrm{CF}, 0.1-0.2 \mathrm{~mL} / \mathrm{min}$ $45 \mathrm{~min}-2 \mathrm{~h} 10$

15 products Diaryl substituted

$65-95 \%$

methyl alcohol

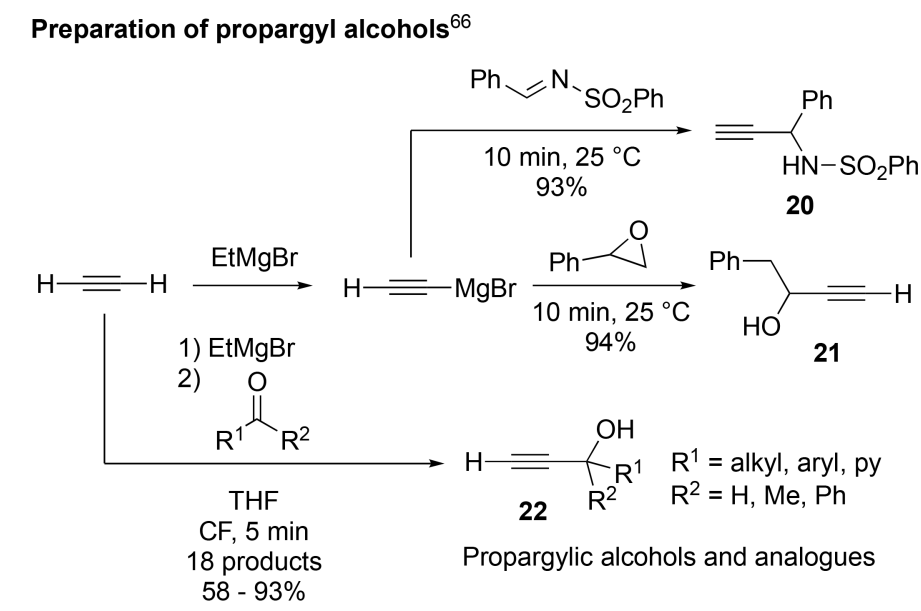


method was developed for the synthesis of Grignard reagent using a fluidized bed reactor. ${ }^{67}$ In the latter case, the formation of the Grignard reagents and their reaction with $\mathrm{CO}_{2}$ could be followed by in-line measurements of NMR spectra.

Continuous processes of lithiation and Grignard reaction were developed and compared in 2016 by the Kopach group (Scheme 5). ${ }^{68}$ Different reactors and procedures were

Scheme 5. Development of Continuous Processes for Grignard and Lithiation for the Synthesis of Benzyl Alcohol

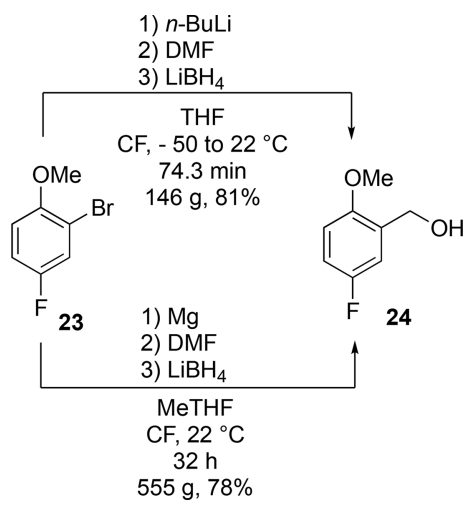

developed to produce the desired benzyl alcohol, using DMF ( $N, N$-dimethylformamide) as the carbonyl source and $\mathrm{LiBH}_{4}$ to reduce the intermediate aldehydes. Similar yields and product purities were obtained with either the lithiation or the Grignard method. Advantages of the lithiation approach include reduced generation of solid (this avoids clogging the system), as well as allowing for a rapid reaction and the ability to use commercially available $n$-BuLi. The lithium halogen exchange, however, generated undesirable 1-bromobutane and butane side products. In contrast, the Grignard method was found to be more atom-economical and safer due to using less hazardous magnesium as opposed to pyrophoric $n$-BuLi. This approach used mild temperatures $\left(20-40{ }^{\circ} \mathrm{C}\right)$, while the lithiation process required working at low temperatures $(-50$ ${ }^{\circ} \mathrm{C}$ ) to avoid side reactions. More recently, the use of less pyrophoric hexyl lithium was reported in a similar halogen/ lithium exchange reaction to perform in-line C-tert-butoxycarbonylation reactions. ${ }^{69}$

Different lithiation reactions were also developed in continuous flow $^{70}$ for the synthesis of various organic compounds (Scheme 6): aryl boronic pinacol ester $31,{ }^{71} \alpha$ keto ester $30,^{72} \alpha$-trifluoromethylamides $33,{ }^{73}$ functionalized aryl fluorides $25,^{74}$ substituted aryl ketone $27,{ }^{75}$ and $\alpha$ haloalcohols $35 .{ }^{76}$ Complexes were generated in situ by reaction of the halide derivate with $n$ - and $s$-BuLi or MeLi and were then reacted with the electrophilic species. Syntheses were highly efficient, yielding the corresponding products in up to $99 \%$ yield in only a few minutes. The aryl boronic pinacol ester 31 was scaled up to $26 \mathrm{~g}$ and was recovered in $99 \%$ yield after $4 \mathrm{~h}$ of total reaction. ${ }^{71}$ Interestingly, thanks to continuous flow and with a similar strategy, different substituted aryl ketones $\mathbf{2 7}$ were synthesized in a protecting-group-free procedure. $^{75}$

\section{Scheme 6. Lithiation Reactions Developed Using the Continuous Flow Process}

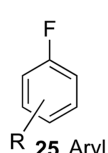

$\mathrm{R}=\mathrm{NO}_{2}, \mathrm{CN}, \mathrm{CO}_{2} \mathrm{tBu}$, $\mathrm{CO}_{2} \mathrm{Et}, \mathrm{OMe}, \mathrm{Me}, \mathrm{SMe}$

$\mathrm{R} 25$ Aryl fluoride

1) $n$-BuLi or PhLi 2) NFSI or $N$-fluorosultam hexane/THF $\mathrm{NFSI}=\left(\mathrm{PhSO}_{2}\right)_{2} \mathrm{NF}$ $45-85 \% \quad \mathrm{~N}$-fluorosultam $=\left(\mathrm{C}_{9} \mathrm{H}_{10} \mathrm{SO}_{2}\right) \mathrm{NF}$ $\mathrm{X}=\mathrm{Br}, 1$
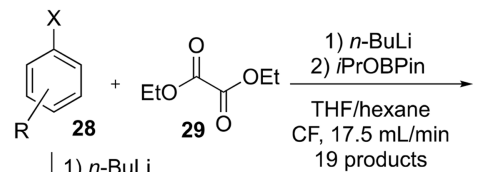

1) $n$-BuLi

2) iPrOBPin THF/hexane

$\mathrm{CF}, 4 \mathrm{~h}$

$26 \mathrm{~g}, 99 \%$

, $\mathrm{X}=\mathrm{Br}$

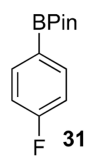

Aryl boronic pinacol ester

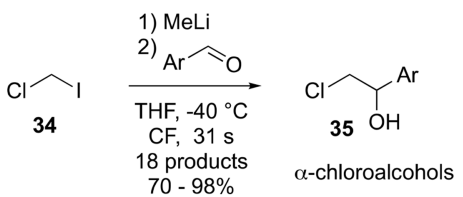

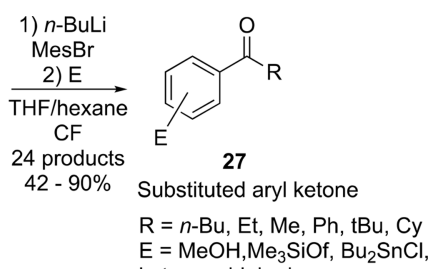

$\mathrm{E}=\mathrm{MeOH}, \mathrm{Me}_{3} \mathrm{SiOf}, \mathrm{Bu}_{2} \mathrm{SnCl}$ ketone, aldehyde

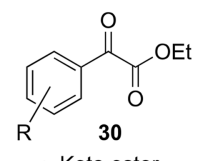

$\alpha$-Keto ester $\mathrm{R}=\mathrm{OMe}, \mathrm{CN}, \mathrm{NO}_{2}, \mathrm{CO}_{2} \mathrm{Et}, \mathrm{CH}_{3}, \mathrm{Br}$, I

$63-95 \%$
$X=B r$

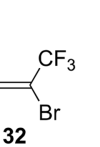

$$
\text { 1) } \mathrm{s}-\mathrm{BuLi}
$$$$
\text { 2) } \mathrm{E}
$$

$\overrightarrow{\mathrm{Et}_{2} \mathrm{O} / \text { hexane }}$

$\mathrm{CF}, 250^{\circ} \mathrm{C}$

$15-20 \mathrm{~min} \quad \mathrm{E}=$ isocyanate

8 products $\mathrm{Nu}=\mathrm{MeOH}$ and malonate ester

$51-80 \%$

$\alpha$-Trifluoromethylamide 


\section{GROUPS 2, 3, 4, AND 5}

\subsection{Mechanochemical Procedures for Complexes of} Groups 2, 3, and 4

Numerous syntheses of metal complexes containing Schiff bases have been described in the literature because of their good catalytic properties in a wide number of reactions including polymerization, reduction, oxidation, and $\mathrm{C}-\mathrm{C}$ coupling reactions. ${ }^{77}$ Interestingly, chiral Schiff-base-containing complexes were more selective in various reactions such as oxidation, hydroxylation, aldol condensation, and epoxidation than complexes featuring other ligands. In addition, Schiff base metal complexes exhibited numerous biological activities such as antifungal, antibacterial, antimalarial, antiproliferative, antiinflammatory, antiviral, and antipyretic properties. ${ }^{78}$ A large number of mechanosyntheses of metal complexes containing Schiff base ligands will be described in the following sections, beginning with zirconium, hafnium, and scandium Schiff base complexes.

Makhaev et al. reported the only mechanosynthesis of group 4 metal complexes containing a Schiff base. These complexes were used to catalyze ethylene polymerization. ${ }^{79}$ Salen complexes 37 with a general formula $\left[\mathbf{M C l}_{2} \mathbf{L}_{2}{ }_{2}\right](\mathrm{M}=\mathrm{Zr}$, Hf and $\mathbf{L}^{1}=N$-(3,5-di-tert-butylsalicylidene)-2,3,5,6-tetrafluoroanilinate anion) were formed starting from a mixture of $N$ (3,5-di-tert-butylsalicylidene)-2,3,5,6-tetrafluoroaniline 36 $\left(\mathrm{HL}^{1}\right), \mathrm{NaH}$, and $\mathrm{MCl}_{4}$, first ground in a mortar and pestle and then transferred to an eccentric vibratory ball mill. The reaction mixture was subjected to grinding for $2 \mathrm{~h}$ at $12 \mathrm{~Hz}$ and then heated at $150{ }^{\circ} \mathrm{C}$ under vacuum to isolate both complexes in a $75-87 \%$ yield (Scheme 7 ). It was found that the reaction

Scheme 7. Mechanochemical Synthesis of $\mathrm{Zr}$ and $\mathrm{Hf}$ Complexes of $\mathrm{N}$-(3,5-Di-tert-butylsalicylidene)-2,3,5,6tetrafluoroanilinate Ligand $\mathrm{L}^{1}$
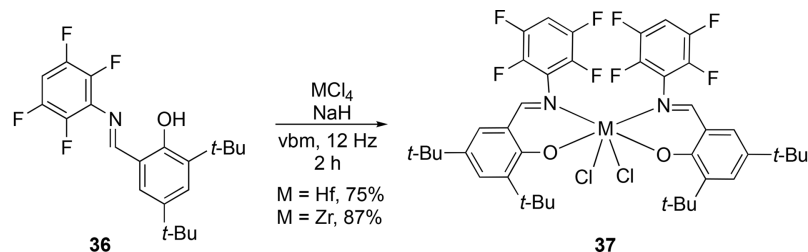

$$
37
$$

was very sensitive to purity and ratio of reagents. Traces of solvent in phenoxyimine led to complete inhibition of the reaction. Even though no explanation was given for this result, the purest complexes were formed by the activation of $\mathrm{LH}-$ $\mathrm{NaH}-\mathrm{MCl}_{4}$ in a 1:1 metal:ligand ratio rather than the 1:2 required by the stoichiometry of the final product. Interestingly, if complexes were synthesized during the milling process, high catalytic properties were observed only after heating the reaction mixture at $150{ }^{\circ} \mathrm{C}$ under vacuum.

During the same year, the mechanosynthesis of tris(allyl)scandium species 39 using a disperser mill or a planetary ball mill was reported (Scheme 8). ${ }^{80} \mathrm{~A}$ disperser mill is composed of a reactor containing a mixing arm and balls, usually made of metal. The rotation at high speed of the mixing arm creates vigorous movements of the balls, leading to a highly efficient mixing of the material placed inside the reactor. Notably, scandium complex 39 in Scheme 8 was unprecedented and never isolated from solution conditions.

In 2016, Boyde et al. performed the mechanosynthesis of Cp (cyclopentadienyl) complexes of titanium 41, zirconium, and
Scheme 8. Mechanosynthesis of Tris(allyl)scandium Species $39^{a}$

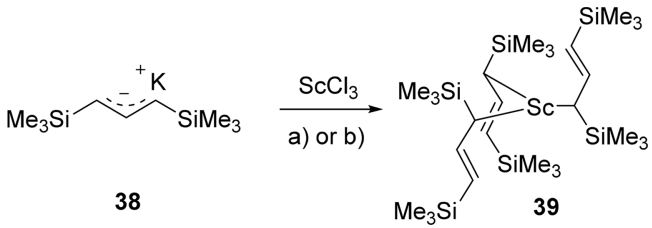

${ }^{a}$ Conditions: (a) disperser milling, $15 \mathrm{~min}, 25 \%$ (around $65 \mathrm{mg}$ scale), (b) pbm, $600 \mathrm{rpm}, 10 \mathrm{~min}, 48 \%$ (410 mg scale).

hafnium using halide metathesis (Scheme 9). Interestingly, starting from $\left[\mathrm{MCp}_{2} \mathrm{Cl}_{2}\right](\mathrm{M}=\mathrm{Zr}$ and $\mathrm{Hf})$, the synthesis led to

Scheme 9. Mechanosynthesis of $\left[\mathrm{TiCp}(\mathrm{Ot}-\mathrm{Bu})_{3}\right]$

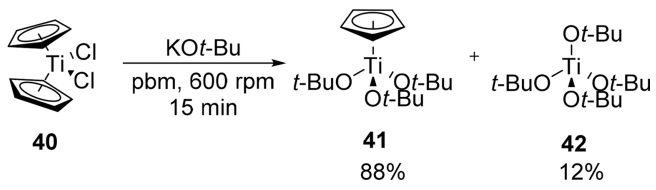

$\left[\mathbf{M C p}_{2}(\mathbf{O} t-\mathbf{B u})_{2}\right]$ complexes; however, starting from the titanium complex 40, the reaction was selective in forming compound $\mathbf{4 1}$ as the major product. ${ }^{81}$ Remarkably, when the synthesis was carried out in hexane, the selectivity of the reaction was similar, but in a polar media such as THF, the synthesis was selective. Using ball milling, reactions could be completed in minutes, whereas hours were required for reactions performed in solution.

Makhaev et al. demonstrated the mechanosynthesis of vanadium(III) $\beta$-diketonates starting from vanadium chloride and sodium $\beta$-diketonates or potassium tetramethylheptanedionate 43 (Scheme 10). ${ }^{82}$ Five products were obtained in $43-$ $85 \%$ yield after 1 to $3 \mathrm{~h}$ of mechanochemical treatment. Interestingly, products could be recovered after sublimation or extraction.

Scheme 10. Mechanosynthesis of Vanadium(III) $\beta$ Diketonates

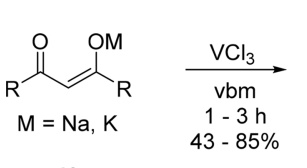

43

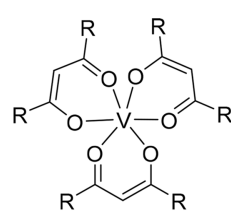

\subsection{Microwave Irradiation for the Synthesis of Vanadium Complexes}

To date there is just a single report of vanadium complex mechanosynthesis. In contrast, synthesis via microwave irradiation is more common. Coordination chemistry and reactivity of vanadium have been studied because of the physiological relevance of the metal and also for its activity in various industrial processes. A number of heterocyclic derivatives containing nitrogen and sulfur atoms such as benzothiazole (one of the most important) serve as a unique and versatile scaffold for experimental drug design. 1,10Phenanthroline (phen, 46) has been extensively used as a ligand due to its high chemical stability, its redox properties, and its good coordination capacity. Alias et al. decided to mix 
Scheme 11. Synthesis of Mixed Benzothiazole/Phenanthroline Vanadium Complexes

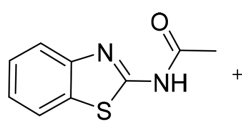

45

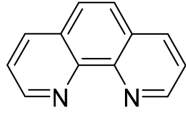

46
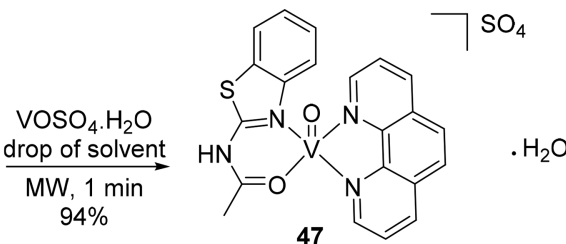

these two ligands in the same complex (Scheme 11). Vanadium(IV) sulfate hydrate, 2-acetamido-1,3-benzothiazole 45, and 1,10-phenanthroline were first mixed in a grinder in a $1: 1: 1$ ratio, and then the reaction mixture was irradiated in the microwave oven using a few drops of solvent. ${ }^{83}$ In 1 min, the corresponding complex 47 was recovered in $94 \%$ yield after a simple filtration using ethanol.

Similarly, heating a solution of $\mathrm{VOSO}_{4} \cdot 5 \mathrm{H}_{2} \mathrm{O}$ with different tetradentate Schiff base ligands 48 in DMF using refluxing conditions or microwave irradiation led to the synthesis of unsymmetrical salen-like vanadium complexes 49 (Scheme 12). ${ }^{84}$ At reflux, 2-3 h was necessary to enable chelation, yet microwave irradiation required only $1 \mathrm{~min}$ to achieve better yields ( $80-84 \%$ in $\mathrm{MW}, 68-70 \%$ in solution).

Scheme 12. Microwave Synthesis of Schiff Base Vanadium Complexes
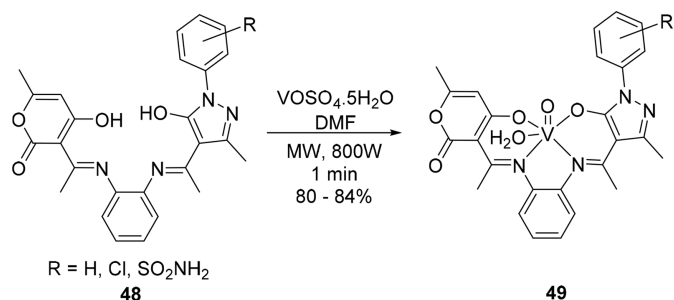

48

\subsection{Synthesis of Vanadium Complexes Using Ultrasound}

Salen-type vanadium complexes $\mathbf{5 2}$ were also synthesized by ultrasound irradiation. Merzougui et al. described the combination of ultrasound irradiation and solvent-free conditions for the synthesis of the tetradentate oxovanadium complex 52. In contrast to classical Schiff base metal syntheses, condensation of diaminoethane 51 and 2-hydroxy-1-naphthaldehyde $\mathbf{5 0}$ was not realized before reaction with the metal source: in this procedure all the reagents, including the metal source, were mixed in a mortar before exposure to ultrasound (Scheme 13). ${ }^{85}$ Ultrasonic activation yielded complex 52 in only $40 \mathrm{~min}$ and in $95 \%$ yield. In contrast, reaction in solution required $3 \mathrm{~h}$ at reflux for a yield of $78 \%$.

\section{GROUP 6}

6.1. Mechanosynthesis of Molybdenum and Chromium Complexes

Molybdenum and chromium complexes exhibit similar properties in reactions such as epoxidation of alkenes. ${ }^{86}$ Due to the rapid hydrolysis of imine ligands in an aqueous solution, the preparation of chromium complexes with ligands containing imine groups remains difficult in aqueous solvent systems. In organic solvents, the insolubility of ligands or metal sources often lowers the reaction efficiency. To avoid this limitation, the Makhaev group proposed the use of mechanical activation for reacting chromium(III) chloride and sodium 4-imino-2pentanonate 53 (NaACIM) in 1994 (Scheme 14). ${ }^{87}$ The

Scheme 14. Synthesis of Chromium Acetylacetonate Derivatives

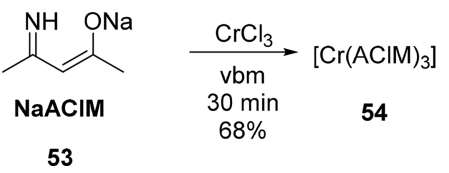

reaction was performed using a vibratory ball mill under nitrogen, and no byproduct was observed. To the best of our knowledge, this report is the only procedure developed for the synthesis of chromium complexes using mechanochemical activation.

Different complexes based on molybdenum possessing the general formula $\left[\mathrm{MoO}_{2}(\mathrm{MSAP})(\mathrm{ROH})\right] \mathbf{5 5}\left(\mathrm{H}_{2} \mathbf{M S A P}=\mathrm{N}-3-\right.$ methoxysalicylidene-2-amino-3-hydroxypyridine, $\mathrm{R}=\mathrm{Me}$, Et, $\mathrm{Pr}$ ) were ground in a mortar and pestle for a few minutes (5$35 \mathrm{~min})$ to afford $\left[\mathrm{MoO}_{\mathbf{2}}(\mathbf{M S A P})\right] \mathbf{5 7}$ with loss of $\mathrm{ROH}$ (Scheme 15, reaction A). ${ }^{88}$ Mortar and pestle were used instead of ball mills to allow the removal of alcoholic vapors from the system. Heating complexes 55 at $200{ }^{\circ} \mathrm{C}$ afforded the polymeric $\left[\mathrm{MoO}_{2}(\mathrm{MSAP})\right]_{n} 56$ which could also be transformed into the monomeric complexes 57 by grinding in a mortar. The same results were reported using the $\mathrm{N}$ salicylidene-2-amino-3-hydroxypyridine Schiff ligand. ${ }^{89}$ In the presence of an $\mathrm{N}$-donating ligand, both these monomeric and polymeric complexes could be transformed into $\left[\mathrm{MoO}_{2}(\mathrm{MSAP})\left(\mathrm{NR}^{\prime}\right)\right] 58$ or $\left[\left(\mathrm{MoO}_{2}(\mathrm{MSAP})\right)_{2}\left(4,4^{\prime}\right.\right.$-bipy $\left.)\right]$ 59 (bipy = bipyridine) complexes using ball milling (Retsch MM200) (Scheme 15, reaction B). Notably, liquid-assisted grinding (LAG) procedures using ACN (acetonitrile) or DCM

Scheme 13. One-Pot Synthesis of Salen Vanadium Complex 52 by Ultrasonic Irradiation

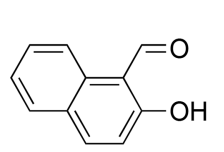

50

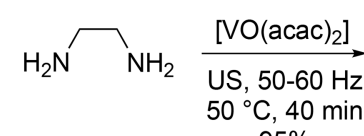

51

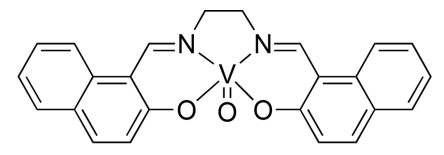

52 
Scheme 15. Mechanochemical Transformation of $\left[\mathrm{MoO}_{2}(\mathrm{MSAP})(\mathrm{ROH})\right]$

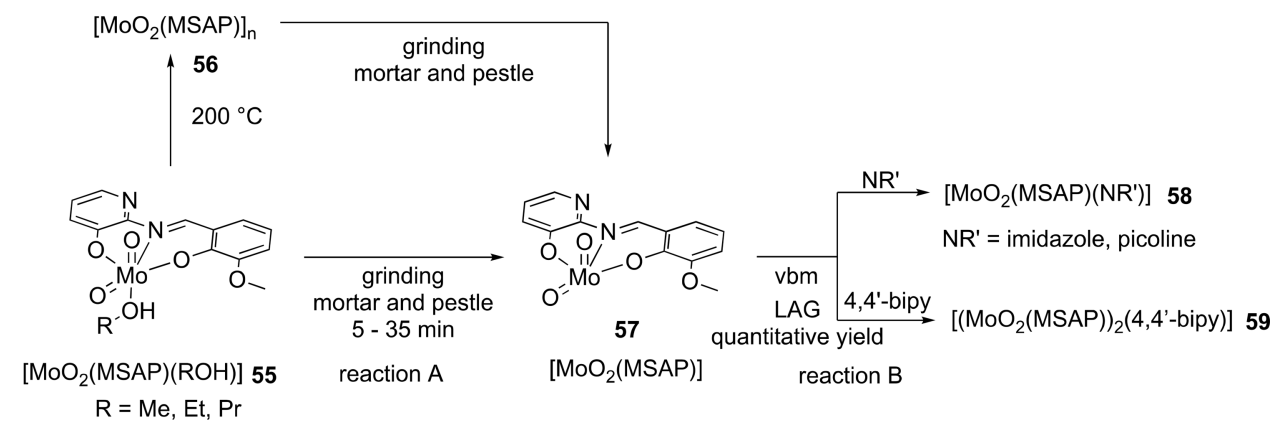

(dichloromethane) allowed isolation of the product in better yields. Using ball milling with an O-donating ligand did not lead to the desired complexes due to the sensitivity of the Moalcohol bond under mechanical treatment.

Nine different molybdenum(IV) O,N,O-Schiff base complexes 61 were mechanosynthesized using a Retsch MM200 vibratory ball mill operated at $25 \mathrm{~Hz}$ (Scheme 16). ${ }^{90}$ Schiff

Scheme 16. Mechanosynthesis of Molybdenum Complexes Using a Liquid Additive

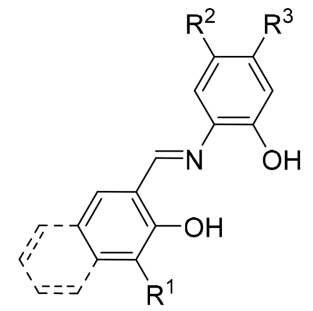

$R^{1}, R^{2}, R^{3}=H, M e, O M e$

60

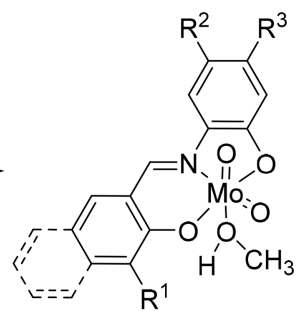

61 base ligands 60 and $\left[\mathrm{MoO}_{2}(\mathrm{acac})_{2}\right]$ (acac = acetylacetonate) were ground in equimolar quantities with a few microliters of $\mathrm{MeOH}$ as a liquid additive. The authors pointed out that these liquid additives played an important role in the stability of the intermediates that led to the formation of the complexes. Catalytic properties of all molybdenum complexes were examined for the epoxidation of cyclooctene, cyclohexene, and limonene. The best results were obtained using the complex coordinated with the Schiff base derived from 2hydroxybenzaldehyde and 2-aminophenol.

\subsection{Microwave Irradiation for the Synthesis of Group 6 Metal Complexes}

As an example of a new procedure using microwave irradiation, the first microwave synthesis of $\left[\mathrm{Cr}(\mathrm{DPM})_{3}\right](\mathrm{DPM}=2,2,6,6$ tetramethyl-3,5-heptadionato) was described in $1990 .^{91} \mathrm{~A}$ solution of $\mathrm{CrCl}_{3} \cdot 3 \mathrm{H}_{2} \mathrm{O}$, urea, and dipivalolylmethane in a mixture of $\mathrm{EtOH}$ and water was heated in a Teflon container under microwave irradiation $(500-600 \mathrm{~W})$ for $40 \mathrm{~s}$ to obtain the complex in $71 \%$ yield. In conventional conditions, the synthesis required $24 \mathrm{~h}$. Consequently, this procedure was definitely an improvement and a milestone for future applications of microwaves in organometallic synthesis.

A series of zerovalent organometallic carbonyl compounds with the general formula $\left[\mathrm{ML}(\mathrm{CO})_{4}\right](\mathrm{M}=\mathrm{Cr}, \mathrm{Mo}$, or W and $\mathrm{L}=1$,2-ethylenediamine 51, dppm 62, dppe 63, and 2,2'-bipy 64) were synthesized under microwave irradiation by Green and co-workers. ${ }^{92}$ Compared to the previously published syntheses performed in solution, reaction times were reduced by a factor of 5 to over 300, and yields were also increased (Scheme 17). Interestingly, reaction times were found to be

\section{Scheme 17. Synthesis of Zerovalent Organometallic} Carbonyl Compounds

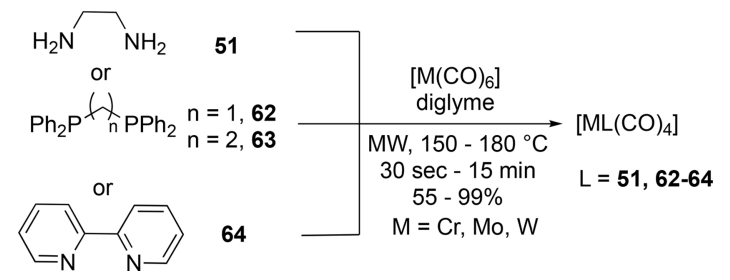

dependent on the metal source without any ligand influence: molybdenum complexes were synthesized in $30 \mathrm{~s}$, chromium complexes in $5 \mathrm{~min}$, and tungsten complexes in $15 \mathrm{~min}$.

To demonstrate the potential of microwave irradiation, 23 organometallic compounds of group 6 were synthesized by the Oscroft group in 2004 (Table 2). ${ }^{93}$ Hexacarbonyl complexes and in particular molybdenum complexes reacted with a range of mono-, bi-, and tridentate $\mathrm{P}$ - and $\mathrm{N}$-coordinating ligands in a modified conventional microwave oven (Table 2, entries 1-10 and 16-23). Interestingly, the selectivity of the reaction between $\mathrm{PPh}_{3}$ and $\left[\mathrm{Mo}(\mathrm{CO})_{6}\right]$ was dependent on the quantity of $\mathrm{PPh}_{3}$ (Table 2, entries 1 and 2). An inert atmosphere was not necessary for the reaction. Better yields and lower reaction times were obtained under microwave irradiation instead of classical refluxing conditions. A further advantage of the microwave synthesis approach was that no sublimation of the parent carbonyl was detected, thereby avoiding a potentially hazardous situation. This is often the case in conventional systems where sublimation can lead to a blockage of the condenser.

More recently, octahedral group 6 carbonyl complexes bearing phosphine or pyridyl ligands were synthesized by coupling borohydride catalyst and microwave heating using a laboratory microwave oven (Scheme 18). ${ }^{94}$ The combination of alcohol solvents and borohydride salts was described to be ideal when combined with microwave heating. Both the hydroxyl group of the alcohol and the borohydride salt strongly absorbed microwaves resulting in a rapid temperature increase. This procedure gave better selectivity than when using solely microwave heating or longer conventional reflux conditions, and the pure cis-[ $\left.\mathbf{M o}\left(\mathbf{P P h}_{3}\right)_{2}(\mathbf{C O})_{4}\right]$ complex was isolated in $73 \%$ yield. Of note, pyridyl complexes were obtained in lower yield than phosphine complexes. 
Table 2. Microwave-Assisted Synthesis of Different Organometallic Complexes of Group 6

\begin{tabular}{|c|c|c|c|c|c|}
\hline entry & substrate & ligand (equiv) & product & time $(\min )^{a}$ & yield (\%) \\
\hline 1 & \multirow{10}{*}[\mathrm{Mo}(\mathrm{CO})_{6}]{} & $\mathrm{PPh}_{3}(1.20)$ & {$\left[\mathrm{Mo}(\mathrm{CO})_{5}\left(\mathrm{PPh}_{3}\right)\right]$} & 40 & 85 \\
\hline 2 & & $\mathrm{PPh}_{3}(2.40)$ & trans- $\left[\mathrm{Mo}(\mathrm{CO})_{4}\left(\mathbf{P P h}_{3}\right)_{2}\right]$ & 80 & 70 \\
\hline 3 & & dppm $62(1.20)$ & $\left.\left[\mathrm{Mo}(\mathrm{CO})_{4}(62)\right]\right]$ & 20 & 87 \\
\hline 4 & & dppe $63(1.20)$ & {$\left[\mathrm{Mo}(\mathrm{CO})_{4}(63)\right.$} & 20 & 96 \\
\hline 5 & & piperidine (excess) & {$\left[\mathrm{Mo}(\mathrm{CO})_{4}\left(\mathrm{NHC}_{5} \mathrm{H}_{10}\right)_{2}\right]$} & 40 & 83 \\
\hline 6 & & 2,2'-bipy 64 (1.20) & $\left.\left[\mathrm{Mo}(\mathrm{CO})_{4}(64)\right]\right]$ & 20 & 94 \\
\hline 7 & & 1,10-phen $46(2.20)$ & $\left.\left[\mathrm{Mo}(\mathrm{CO})_{4}(46)\right]\right]$ & 15 & 91 \\
\hline 8 & & pyridine (excess) & $c i s-\left[\mathrm{Mo}(\mathrm{CO})_{4}(\mathrm{py})_{2}\right]$ & 20 & 50 \\
\hline 9 & & dicyclopentadiene (2.00) & {$\left[\mathrm{MoCp}(\mathrm{CO})_{3}\right]_{2}$} & 60 & 94 \\
\hline 10 & & acetic acid (excess) & {$\left[\mathrm{Mo}_{2}(\mathrm{OAc})_{4}\right]$} & 45 & 48 \\
\hline 11 & {$\left[\mathrm{Mo}(\mathrm{CO})_{4}(64)\right]$} & 2,2'-bipy $(2.15)$ & $c i s-\left[\mathrm{Mo}(\mathrm{CO})_{2}(64)_{2}\right]$ & 90 & 53 \\
\hline 12 & \multirow[t]{2}{*}[\mathrm{Mo}(\mathrm{CO})_{4}(62)]{} & dppm $62(1.20)$ & $c i s-\left[\mathrm{Mo}(\mathrm{CO})_{2}(62)_{2}\right]$ & 40 & 63 \\
\hline 13 & & dppe $63(1.20)$ & $c i s-\left[\mathrm{Mo}(\mathrm{CO})_{2}(62)(63)\right]$ & 40 & 52 \\
\hline 14 & \multirow{7}{*}{$\begin{array}{l}{\left[\mathrm{Mo}(\mathrm{CO})_{4}(63)\right]} \\
{\left[\mathrm{MoCp}(\mathrm{CO})_{3}\right]_{2}} \\
{\left[\mathrm{~W}(\mathrm{CO})_{6}\right]}\end{array}$} & dppe $63(1.20)$ & $c i s-\left[\mathrm{Mo}(\mathrm{CO})_{2}(63)_{2}\right]$ & 40 & 45 \\
\hline 15 & & diphenylacetylene (3.00) & {$\left[\mathrm{Mo}_{2} \mathrm{Cp}_{2}(\mathrm{CO})_{4}\left(\mu-\mathrm{PhC}_{2} \mathrm{Ph}\right)\right]$} & 120 & 39 \\
\hline 16 & & $\mathrm{PPh}_{3}(2.40)$ & {$\left[\mathrm{W}(\mathrm{CO})_{4}\left(\mathrm{PPh}_{3}\right)_{2}\right]$} & 20 & 96 \\
\hline 17 & & dppm $62(0.90)$ & {$\left[\mathrm{W}(\mathrm{CO})_{4}(62)\right]$} & 20 & 68 \\
\hline 18 & & piperidine (excess) & $c i s-\left[\mathrm{W}(\mathrm{CO})_{4}(\text { piperidine })_{2}\right]$ & 120 & 35 \\
\hline 19 & & 2,2'-bipy 64 (1.20) & {$\left[\mathrm{W}(\mathrm{CO})_{4}(64)\right]$} & 80 & 39 \\
\hline 20 & & 1,10-phen $46(2.00)$ & {$\left[\mathrm{W}(\mathrm{CO})_{4}(46)\right]$} & 60 & 85 \\
\hline 21 & \multirow[t]{3}{*}{$\mathrm{Cr}(\mathrm{CO})_{6}$} & piperidine (excess) & {$\left[\mathrm{Cr}(\mathrm{CO})_{5}(\right.$ piperidine $\left.)\right]$} & 40 & 14 \\
\hline 22 & & $\mathrm{Et}_{4} \mathrm{NCl} \cdot \mathrm{H}_{2} \mathrm{O}(1.10)$ & {$\left[\mathrm{Cr}(\mathrm{CO})_{5} \mathrm{Cl}\right]\left[\mathrm{NEt}_{4}\right]$} & 30 & 74 \\
\hline 23 & & anisole (excess) & {$\left[\mathrm{Cr}\left(\eta^{6}-\mathrm{C}_{6} \mathrm{H}_{5} \mathrm{OMe}\right)(\mathrm{CO})_{3}\right]$} & 240 & 45 \\
\hline
\end{tabular}

${ }^{a}$ Microwave syntheses were carried out in a flask equipped with a water condenser and placed in a modified PROLINE program $1250750 \mathrm{~W}$ microwave oven. $\mathrm{dppm}=$ bis $($ diphenylphosphino $)$ methane. dppe $=$ bis (diphenylphosphino $)$ ethane.

Scheme 18. Microwave-Assisted Synthesis of Group 6 Complexes Using $\mathrm{NaBH}_{4}$ as Catalyst

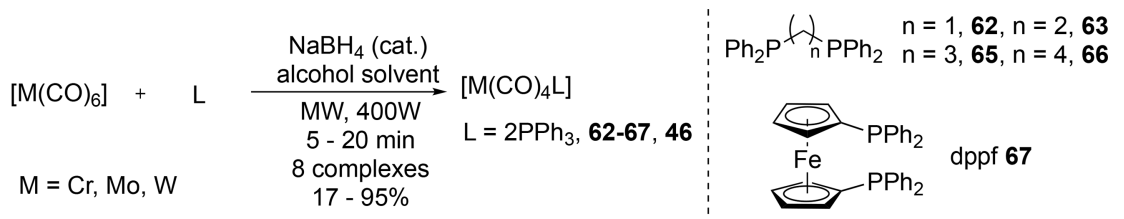

Scheme 19. Synthesis of Mixed Ligand Chromium Complexes

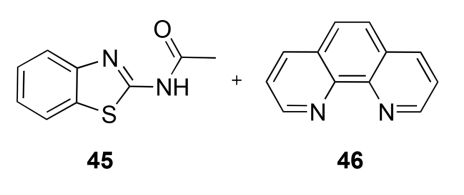

In 2015, and as they did for the synthesis of vanadium complexes (see part 5.2, Scheme 11), Alias et al. described the microwave synthesis of the mixed phenanthroline and 2acetamide benzothiazole chromium complex 68 that was produced after $80 \mathrm{~s}$ of heating and isolated in $82 \%$ yield (Scheme 19). ${ }^{83}$

Between 2010 and 2016, the Jain group described the microwave synthesis of different chromium complexes with Schiff base ligands which were further used for their antimicrobial properties. $\left[\mathrm{Cr}(74)\left(\mathrm{H}_{2} \mathrm{O}\right)_{3}\right] \mathrm{Cl}^{95}[\mathrm{Cr}(75) \mathrm{Cl}]$. $\mathbf{2 H}_{\mathbf{2}} \mathbf{O},{ }^{95}\left[\mathrm{Cr}(\mathbf{7 8})_{2}\left(\mathbf{H}_{\mathbf{2}} \mathrm{O}\right)_{2}\right] \cdot \mathbf{C l},^{96}\left[\mathbf{C r}(\mathbf{8 0})_{2}\left(\mathbf{H}_{\mathbf{2}} \mathbf{O}\right)_{2}\right] \mathbf{C l},{ }^{96}[\mathbf{C r}-$ $\left.(84)_{2}\left(\mathrm{H}_{2} \mathrm{O}\right)_{2}\right] \mathrm{Cl} \cdot 2 \mathrm{H}_{2} \mathrm{O},{ }^{97}\left[\mathrm{Cr}(83)\left(\mathrm{H}_{2} \mathrm{O}\right)_{3}\right] \mathbf{C l}_{2},{ }^{97}[\mathrm{Cr}(88)-$ $\left.\left(\mathbf{H}_{2} \mathbf{O}\right) \mathbf{C l}\right],{ }^{98}\left[\mathrm{Cr}(\mathbf{8 1})_{2}\left(\mathbf{H}_{2} \mathbf{O}\right)_{2}\right] \cdot \mathrm{Cl}^{98}\left[\mathrm{Cr}(\mathbf{8 2})_{2}\left(\mathbf{H}_{2} \mathbf{O}\right)_{2}\right] \cdot \mathbf{C l},{ }^{99}$ $\left[\mathrm{Cr}(\mathbf{8 5})_{2}\left(\mathrm{H}_{2} \mathrm{O}\right)_{2}\right] \mathrm{Cl}^{100}$ and $\left[\mathrm{Cr}(\mathbf{8 6})_{2}\left(\mathbf{H}_{\mathbf{2}} \mathrm{O}\right)_{2}\right] \mathbf{C l}^{100}$ were synthesized as follows. Chromium salt and ligand were ground together and then transferred into a reactor with $3-5 \mathrm{~mL}$ of

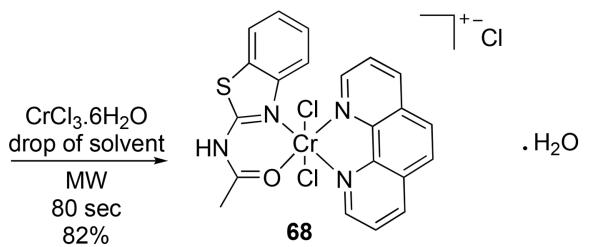

dry EtOH to be heated under microwave irradiation (Table 3, entries 1-11; see Figure 1 for ligand structure). All products were recovered in higher yields and lower reaction times than when using conventional reflux conditions; reaction times did not exceed $14 \mathrm{~min}$, while yields ranged from 57 to $87 \%$.

The Fahmi group has also described the synthesis of Schiff base chromium complexes via microwave irradiation starting from $\mathrm{CrCl}_{3}$ and ligand $\mathbf{7 2}$ or 73 (Table 3). ${ }^{101}$ Changing the metal:ligand ratio led to the selective formation of different complexes. With a 1:1 ratio, $\left[\mathrm{CrCl}(\mathrm{L})\left(\mathrm{H}_{2} \mathrm{O}\right)_{2}\right]$ complexes were formed (Table 3, entries 12 and 14), while with a 1:2 ratio, $\left[\mathrm{CrCl}(\mathrm{L})_{2}\left(\mathrm{H}_{2} \mathrm{O}\right)\right]$ complexes were obtained (Table 3, entries 13 and 15). In all cases, since concentration was increased in the procedure utilizing microwave irradiation, this led to a difficult comparison with the classical solution procedure. Ligand and metal complexes have been screened 
Table 3. Synthesis of Schiff Base Chromium Complexes ${ }^{a}$

\begin{tabular}{|c|c|c|c|c|c|c|}
\hline \multirow[b]{2}{*}{ entry } & \multirow[b]{2}{*}{ chromium complex } & \multicolumn{2}{|c|}{$\begin{array}{l}\text { microwave } \\
\text { synthesis }\end{array}$} & \multicolumn{2}{|c|}{$\begin{array}{l}\text { conventional } \\
\text { heating }\end{array}$} & \multirow[b]{2}{*}{ ref } \\
\hline & & $\begin{array}{l}\text { time } \\
(\mathrm{min})\end{array}$ & $\begin{array}{l}\text { yield } \\
(\%)\end{array}$ & $\begin{array}{l}\text { time } \\
(\mathrm{h})\end{array}$ & $\begin{array}{l}\text { yield } \\
(\%)\end{array}$ & \\
\hline 1 & {$\left[\mathrm{Cr}(74)\left(\mathrm{H}_{2} \mathrm{O}\right)_{3}\right] \mathrm{Cl}$} & 8 & 80 & 8 & 60 & 95 \\
\hline 2 & {$[\mathrm{Cr}(75) \mathrm{Cl}] \cdot 2 \mathrm{H}_{2} \mathrm{O}$} & 8 & 78 & 8 & 60 & 95 \\
\hline 3 & {$\left[\mathrm{Cr}(78)_{2}\left(\mathrm{H}_{2} \mathrm{O}\right)_{2}\right] \cdot \mathrm{Cl}$} & 9 & 79 & 8 & 61 & 96 \\
\hline 4 & {$\left[\mathrm{Cr}(80)_{2}\left(\mathrm{H}_{2} \mathrm{O}\right)_{2}\right] \mathrm{Cl}$} & 10 & 78 & 8 & 59 & 96 \\
\hline 5 & 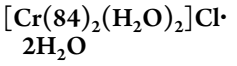 & 9 & 78 & 8 & 60 & 97 \\
\hline 6 & {$\left[\mathrm{Cr}(83)\left(\mathrm{H}_{2} \mathrm{O}\right)_{3}\right] \mathrm{Cl}_{2}$} & 8 & 76 & 8 & 57 & 97 \\
\hline 7 & {$\left[\mathrm{Cr}(88)\left(\mathrm{H}_{2} \mathrm{O}\right) \mathrm{Cl}\right]$} & 9 & 79 & 10 & 62 & 98 \\
\hline 8 & {$\left[\mathrm{Cr}(81)_{2}\left(\mathrm{H}_{2} \mathrm{O}\right)_{2}\right] \cdot \mathrm{Cl}$} & 8 & 80 & 8 & 58 & 99 \\
\hline 9 & {$\left[\mathrm{Cr}(82)_{2}\left(\mathrm{H}_{2} \mathrm{O}\right)_{2}\right] \cdot \mathrm{Cl}$} & 9 & 75 & 9 & 61 & 99 \\
\hline 10 & {$\left[\mathrm{Cr}(85)_{2}\left(\mathrm{H}_{2} \mathrm{O}\right)_{2}\right] \mathrm{Cl}$} & 9 & 77 & 9 & 61 & 100 \\
\hline 11 & {$\left[\mathrm{Cr}(86)_{2}\left(\mathrm{H}_{2} \mathrm{O}\right)_{2}\right] \mathrm{Cl}$} & 8 & 79 & 9 & 65 & 100 \\
\hline 12 & {$\left[\mathrm{CrCl}(72)\left(\mathrm{H}_{2} \mathrm{O}\right)_{2}\right]$} & 10 & 83 & 15 & 73 & 101 \\
\hline 13 & {$\left[\mathrm{CrCl}(72)_{2}\left(\mathrm{H}_{2} \mathrm{O}\right)\right]$} & 10 & 87 & 14 & 87 & 101 \\
\hline 14 & {$\left[\mathrm{CrCl}(73)\left(\mathrm{H}_{2} \mathrm{O}\right)_{2}\right]$} & 12 & 80 & 16 & 70 & 101 \\
\hline 15 & {$\left[\mathrm{CrCl}(73)_{2}\left(\mathrm{H}_{2} \mathrm{O}\right)\right]$} & 14 & 84 & 14 & 77 & 101 \\
\hline
\end{tabular}

${ }^{a}$ See Figure 1 for the structure of ligands.

in vitro for antimicrobial activity and in vivo for antifertility activity on male albino rats. Of note, the complexes are more potent than the parent ligands.

Similarly, a series of Salen-type chromium complexes were synthesized using microwave irradiation starting from tetradentate schiff base ligands 107 (Scheme 20). ${ }^{102}$ Complexes 108 were obtained rapidly in good yields and exhibited antimicrobial activity.

Interestingly, Whittaker and Mingos described different reactions under microwave irradiation, in a modified domestic oven, using metal powder. ${ }^{103}$ Thus, chromium powder was transformed into a sandwich organometallic species 110 in reasonable yield (52\%) when treated with $\mathrm{AlCl}_{3}$, toluene, and $\mathrm{HCl}$ gas (Scheme 21, pathway A). The same complex as well as 109, a similar one derived from benzene, could be synthesized using a mixture of aluminum powder, $\mathrm{AlCl}_{3}$, and $\mathrm{CrCl}_{3}$ (Scheme 21, pathway B). Reactions were not chosen to display any significant increase in reaction rate and/or yields but rather to demonstrate the possibility of using metal powder under microwave irradiations. Despite this, reaction times were considerably reduced from hours to only a few minutes using microwave irradiation instead of classical solution conditions. Although utilization of zerovalent metal powders is known to be highly reactive under microwave irradiations, no peculiar incident and/or process deviation were reported by the authors.

The conventional synthesis of $\left(\eta^{6}\right.$-arene $)$ tricarbonylchromium complexes was described as being limited by the high temperature and long reaction time required. To overcome this hurdle, many useful complexation reagents were developed for the synthesis of arene chromium complexes such as $\left[\mathrm{Cr}(\mathrm{CO})_{3}(\mathrm{~L})_{3}\right] 111\left(\mathrm{~L}=\right.$ py, $\mathrm{NH}_{3}, \eta^{6}$-naphthalene, and ACN which were considered as labile ligands). These procedures required a step involving derivatization of chromium hexacarbonyl into 111. This derivatization was shown to be unnecessary when using microwave irradiation for the reaction of $\left[\mathrm{Cr}(\mathrm{CO})_{6}\right]$ and various arenes. The procedure produced the complexes in $1 \mathrm{~h}$ and in $48-79 \%$ yields while being economical in atoms and steps (Scheme 22). ${ }^{104}$ Interestingly, this strategy was applied to the synthesis of
112, a tricarbonylchromium complex of a steroid-like compound.

Peroxo complexes and in particular oxodiperoxo molybdenum complexes bearing bidentate ligands are widely used in the epoxidation of olefins. The oxodiperoxo complex [MoO$\left(\mathrm{O}_{2}\right)_{2}$ (tbbpy)] 114 (tbbpy $=4,4^{\prime}$-di-tert-butyl-2,2' -bipyridine) was isolated from the reaction of $\left[\mathrm{MoO}_{2} \mathrm{Cl}_{2}(\mathbf{t b b p y})\right] 113$ in water under microwave irradiation at $120{ }^{\circ} \mathrm{C}$ for $4 \mathrm{~h}$ (Scheme 23). ${ }^{105}$ Of note, it was the first report describing the isolation of this type of complex in the absence of a highly oxidant species such as $\mathrm{H}_{2} \mathrm{O}_{2}$ or peroxide. Oxidation was only due to atmospheric oxygen. When the same reaction was carried out under classical reflux conditions, no formation of the oxodiperoxo complex was observed.

Due to their potential relevance as model systems for molybdenum enzymes, numerous papers reported the synthesis of metallacyclic oxomolybdenum complexes. As for vanadium oxide described earlier (see section 6.2), a novel synthesis of unsymmetrical Schiff base complexes with oxocation of molybdenum was realized using classical stirring and microwave technology. In solution, $8-10 \mathrm{~h}$ of stirring was generally necessary to afford the desired complexes in 59-61\% yield. When performing the same reaction under microwave irradiation, reaction time was decreased to $1 \mathrm{~min}$, and complexes 115 were recovered in $77-79 \%$ yield (Scheme 24). ${ }^{84}$

cis- $\left.\left[\mathbf{M o}(\mathbf{C O})_{4} \text { (piperidine }\right)_{2}\right] \quad 117^{106}$ and cis-[Mo$(\mathrm{CO})_{4}($ pzpy $\left.)\right] \mathbf{1 1 8}^{107}$ (pzpy $=$ ethyl[3-(2-pyridyl)-1pyrazolyl $]$ acetate $)$ were synthesized by reacting $\left[\mathrm{Mo}(\mathrm{CO})_{6}\right]$ under microwave irradiation with piperidine and pzpy 116, respectively (Scheme 25, reactions $\mathrm{A}, \mathrm{B}$, and $\mathrm{C}$ ). Complexes were recovered in shorter reaction times and in higher yields than when obtained by synthesis in solution. Interestingly, due to the lability of the amine ligand, simple ligand substitution reactions in 117 could be carried out using various nucleophiles such as triphenylphosphine. Notably, the synthesis of 118 under microwave irradiation enabled shortening of the synthetic scheme by one step. In solution, 118 had to be synthesized by ligand substitution of 117 .

Optimization of organometallic complex production via microwave irradiation is generally limited by the necessity to carry out ex situ analysis of the reaction mixture, leading to laborious and time-consuming step-by-step optimization. To solve this problem, Barnard and Leadbeater developed equipment for real-time monitoring of organometallic reactions under microwave irradiation using in situ Raman spectroscopy. ${ }^{108}$ This setup was utilized to efficiently monitor the ligand substitution reactions of $\left[\mathrm{Mo}(\mathrm{CO})_{6}\right]$. Thus, the formation of mono- $\left[\mathrm{Mo}(\mathrm{CO})_{5} \mathrm{~L}\right]$, bi- $\left[\mathrm{Mo}(\mathrm{CO})_{4} \mathrm{~L}_{2}\right]$, and tri$\left[\mathrm{Mo}(\mathrm{CO})_{3} \mathrm{~L}_{3}\right]$ species could be detected and analyzed by in situ Raman spectroscopy during the course of the reactions (ligand $=$ Py, octylamine, and $\mathrm{PPh}_{3}$ ).

Molybdenum complex 118 (Scheme 25) and cis-[W$(\mathrm{CO})_{4}$ (pzpy)] 119 (Scheme 26) were synthesized under microwave irradiation from ligand 116 with $\left[\mathrm{Mo}(\mathrm{CO})_{6}\right]$ and $\left[\mathrm{W}(\mathrm{CO})_{6}\right]$, respectively. Tungsten complex 119 was obtained after $15 \mathrm{~min}$ irradiation, with longer reaction times required compared to that of molybdenum complex 118 due to the lower reactivity of $\left[\mathbf{W}(\mathbf{C O})_{6}\right]$ compared to $\left[\mathbf{M o}(\mathbf{C O})_{6}\right](15$ min instead of $30 \mathrm{~s}) .^{107}$

Incorporation of an organometallic functional group into a biomolecule to modify its properties has been developed recently. For instance, tungsten-containing pyrimidines such as 

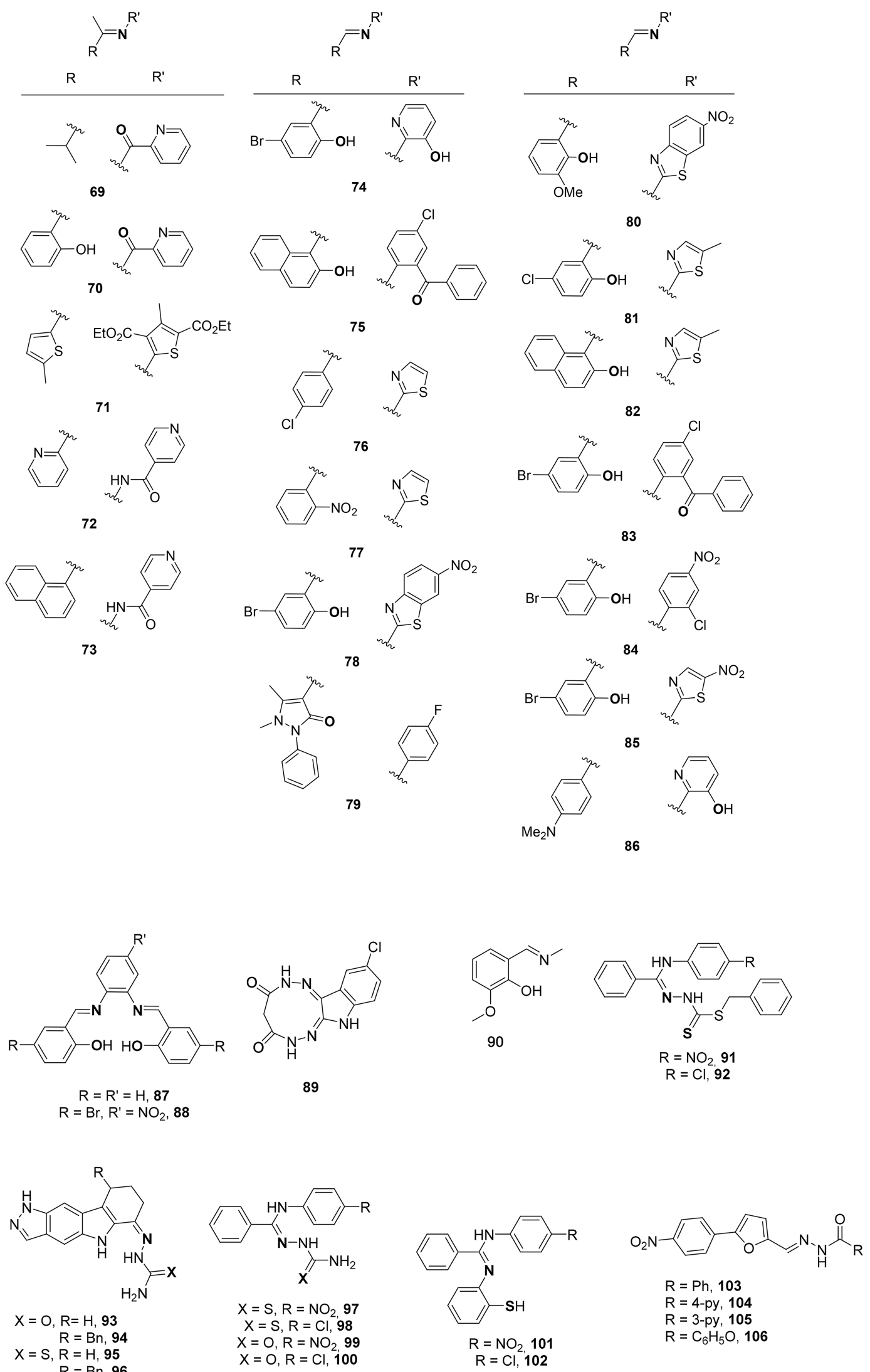

Figure 1. Schiff base ligand used for the synthesis of metal complexes under microwave irradiation.

121-123 have been interesting for their biological properties; compounds were identified as ethologically active compounds in the ponerine ant Megaponera fetens. Their syntheses were described in solution by reaction of alkynyl alkoxy carbene 
Scheme 20. Synthesis of Salen-Type Chromium Complexes Using Microwave Irradiation

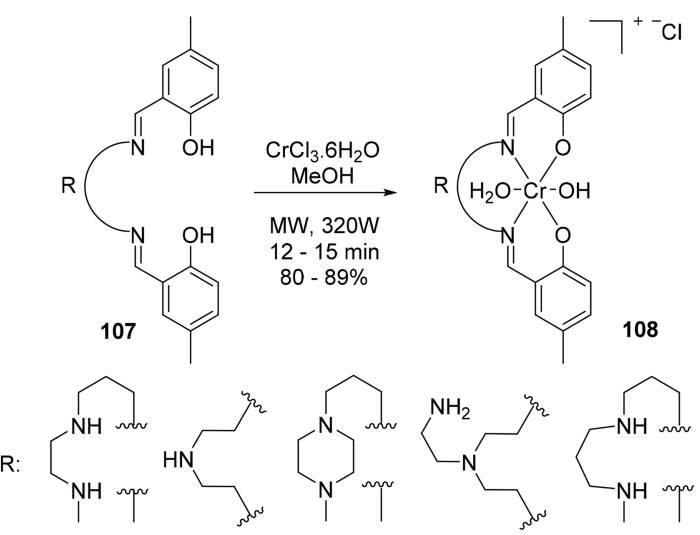

Scheme 21. Sandwich Chromium Species Formation under Microwave Irradiation

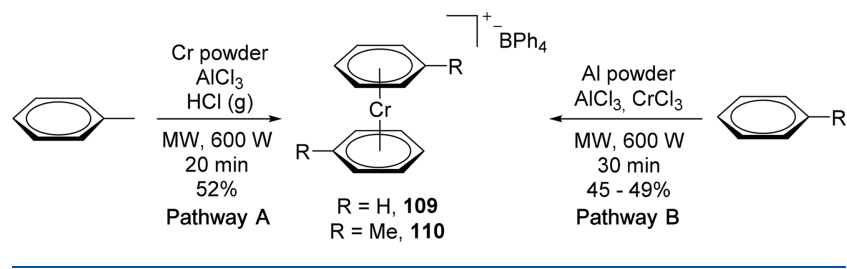

metal complexes with mono- and dimethyl ureas, furnishing the heterocycles in high yields but in long reaction times. By using microwave irradiation, reaction time was considerably reduced from hours (at room temperature) to a few minutes (at $60{ }^{\circ} \mathrm{C}$ ) (Scheme 27). ${ }^{109}$ Despite this, yields were in the same range as conventional solution-based synthesis. Change of the acetylene moiety was studied: a trimethylsilylsubstituted alkynyl carbene complex was used, but low yields were obtained due to the easy desilylation of the starting material.

\subsection{Photochemistry for Synthesis of Group 6 Metal Complexes}

Photochemical ligand substitution of group 6 metal complexes is a well-known reaction. Mechanism investigations demonstrated that labile ligands were easily removed at the beginning of the reaction, in particular CO ligands that were released from the reaction as gas. An exogenous ligand could further react with the intermediate photolyzed species. In this part, different substitutions will be described. Two different photochemical methods for the synthesis of arene-chromium $\left[\mathrm{Cr}(\right.$ arene $\left.)(\mathbf{C O})_{3}\right] 111$ were developed in 1989. The first involved irradiation with a mercury lamp of a $\left[\mathrm{Cr}(\mathrm{CO})_{6}\right]$ solution and arene in THF (Scheme 28, reaction A), while the second proceeded by the same irradiation of a suspension of
Scheme 23. Synthesis of Oxodiperoxo Molybdenum Complex 114 Using Air as an Oxidant

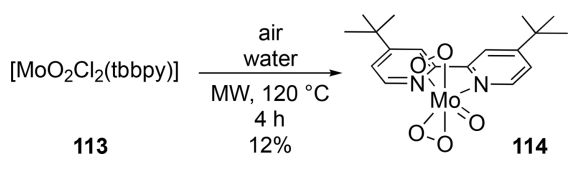

Scheme 24. Schiff Base Complexes of Molybdenum Oxide Synthesized under Microwave Irradiation

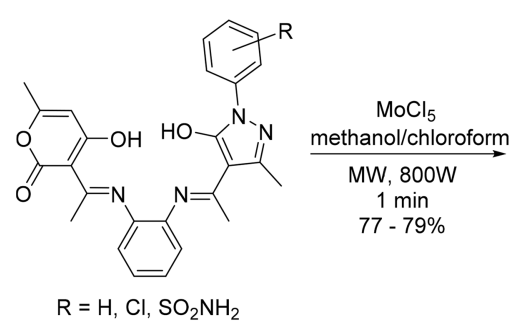

48

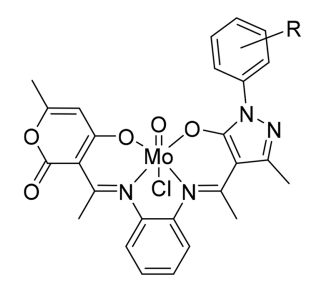

115
Scheme 25. Microwave Transformation of $\left[\mathrm{Mo}(\mathrm{CO})_{6}\right]$ under Microwave Irradiation

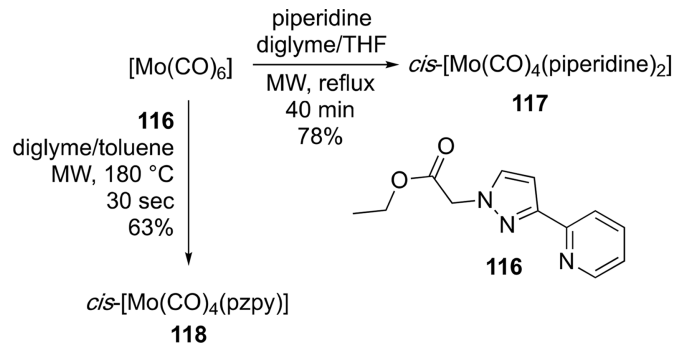

Scheme 26. $\left[\mathrm{W}(\mathrm{CO})_{4}(\mathrm{pzpy})\right]$ Formation under Microwave Irradiation

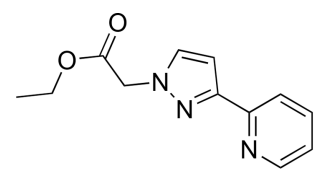

116

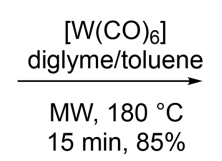

$15 \min , 85 \%$

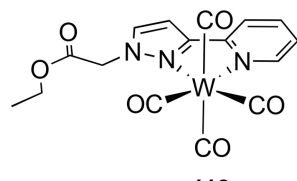

119
$\left[\mathrm{Cr}(\mathrm{CO})_{6}\right]$ in THF before adding the arene in the reaction mixture (Scheme 28, reaction B). ${ }^{110}$ Different arenes were used (e.g., benzene, halobenzene, anisole, $N, N$-dimethylaniline, and methylbenzoate, etc.), and interestingly, thermal sensitive arene-containing substrates resulted in the corresponding products with satisfying yields. Notably, yields were lower than those obtained classically via thermal solvent-based methods; yet the milder reaction conditions under photochemical conditions gave an additional advantage to this protocol. Significantly, this procedure was effectively applied to

Scheme 22. Microwave Synthesis of Arene Chromium Complexes

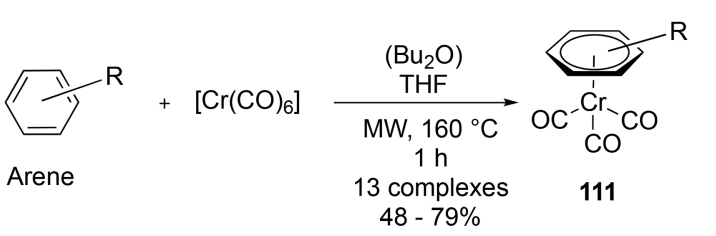

Arene $=$ benzene, toluene, anisole, mesitylene, biphenyl, fluorene...

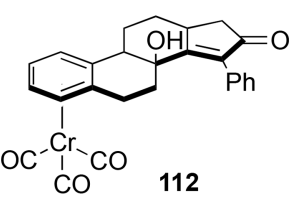

Tricarbonylchromium complex of a steroid-like compounds 
Scheme 27. Microwave-Assisted Synthesis of Tungsten Uracil-Analogue Fisher Carbene Complexes

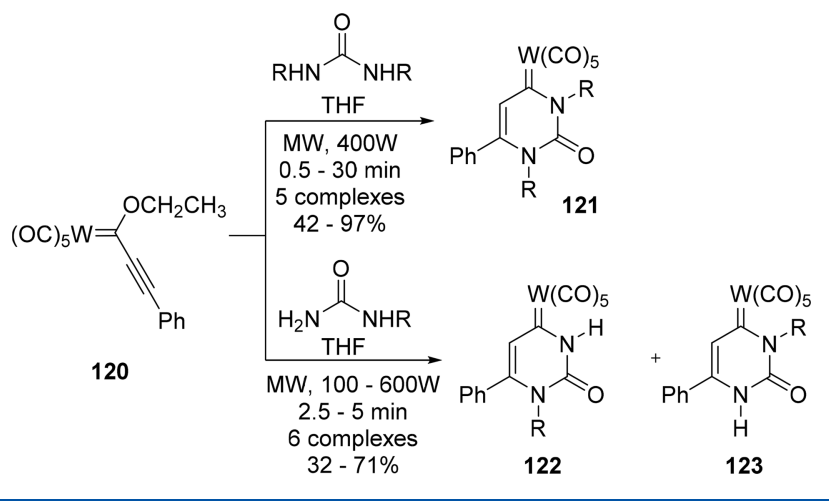

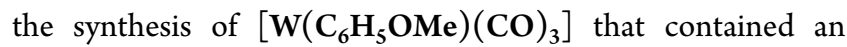
electron-rich arene and which could be recovered in $24 \%$ yield (Scheme 29, reaction A). Moreover, the irradiation of $[\mathrm{Cr}$ (benzene $\left.)(\mathrm{CO})_{3}\right]$ in the presence of an excess of piperidine led to the formation of the $\left[\mathrm{Cr}(\right.$ piperidine $\left.)(\mathrm{CO})_{3}\right]$ complex. $^{111}$

Preparation of hexaalkylborazole chromium complexes could not be achieved by thermal activation. The reaction was consistently unsuccessful despite considerable efforts to make it succeed. Contrastingly, photolysis under reduced pressure of $\left[\mathrm{Cr}(\mathrm{CO})_{6}\right]$ in the presence of hexaalkylborazole ligands led to the formation of the desired complexes in excellent yields (Scheme 28, reaction C). ${ }^{112}$ Moreover, half-sandwich-type complexes with the general formula $\left[\mathrm{CrL}(\mathrm{CO})_{3}\right](\mathrm{L}=$ $\left(\mathrm{C}_{2} \mathrm{H}_{5}\right)_{3} \mathrm{~B}_{3} \mathrm{~N}_{3}\left(\mathrm{CH}_{3}\right)_{3}, \quad\left(\mathrm{CH}_{3}\right)_{3} \mathrm{~B}_{3} \mathrm{~N}_{3}\left(\mathrm{C}_{2} \mathrm{H}_{5}\right)_{3}, \mathrm{C}_{6} \mathrm{H}_{6}, \mathrm{C}_{5} \mathrm{H}_{5} \mathrm{~N}$, $\mathrm{C}_{6} \mathrm{H}_{5} \mathrm{CN}$, and mesitylene) could also be synthesized by photochemical reaction of $\left[\mathrm{Cr}(\mathrm{CO})_{6}\right]$ in the presence of the ligand under reduced pressure. Complexes were recovered in $80-90 \%$ yields after only $1 \mathrm{~h}$ of irradiation using a mercury vapor lamp $(125 \mathrm{~W})$. Interestingly, reactions were performed without the use of a traditional bulk solvent. They were replaced by the ligands that were liquid at room temperature and used in excess. Moreover, reactions performed under atmospheric pressure failed, and this failure could be explained by the nonfavorable equilibrium of nucleophilic substitution under these conditions. In order to shift the equilibrium of the reaction toward the formation of the complex, CO ligands had to be removed under reduced pressure. Starting from the same chromium source, irradiation with a mercury vapor lamp of a solution of $\left[\mathrm{Cr}(\mathrm{CO})_{6}\right]$ in acetonitrile led to the photochemical substitution of two carbonyls by the acetonitrile ligand in 20
Scheme 29. Photochemical Transformation of $\left[\mathrm{W}(\mathrm{CO})_{6}\right]$

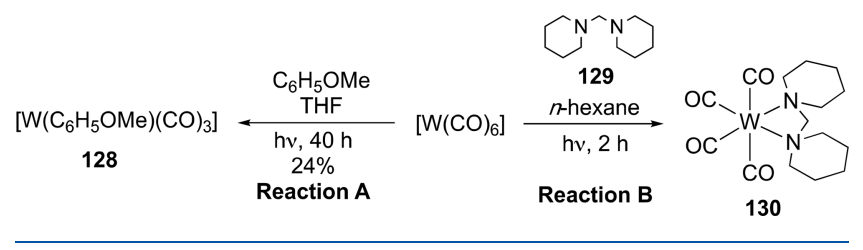

min, leading to the formation of cis- $\left[\mathrm{Cr}(\mathrm{CO})_{4}(\mathrm{ACN})_{2}\right] \mathbf{1 2 4}$ (Scheme 28 , reaction $\mathrm{D}) .{ }^{113}$ While shorter reaction times led to incomplete conversion and formation of the monosubstituted complex, the $f a c$ - $\left[\mathrm{Cr}(\mathbf{C O})_{3}(\mathbf{A C N})_{3}\right]$ complex started to be detected after more than $30 \mathrm{~min}$ irradiation. In comparison, the reaction under thermal conditions led to the formation of the complex after $24 \mathrm{~h}$. The product was, however, contaminated by both the mono- and the trisubstituted derivatives. Complex 124 (cis- $\left[\mathrm{Cr}(\mathrm{CO})_{4}(\mathrm{ACN})_{2}\right]$ ) could then be transformed to cis- $\left[\mathrm{Cr}(\mathrm{CO})_{4}(\mathrm{dppm})\right] \mathbf{1 2 6}$ in $65 \%$ and to trans- $\left[\mathbf{C r}(\mathbf{C O})_{4}\left(\mathbf{P P h}_{3}\right)_{2}\right] \quad \mathbf{1 2 5}$ in $79 \%$ yield after conventional heating with dppm 62 and $\mathrm{PPh}_{3}$, respectively.

Photolysis of $\left[\mathrm{Cr}(\mathrm{CO})_{6}\right]$ could also be performed under pressure of propene $(1370 \mathrm{psi})$, obtaining $\left[\mathrm{Cr}(\mathbf{C O})_{5}\left(\boldsymbol{\eta}^{2}\right.\right.$ $\left.\left.\mathrm{C}_{3} \mathrm{H}_{6}\right)\right] 127$ in $60 \%$ yield. Under pressure, propene was transformed into a liquid that acted as both a reagent and a solvent (Scheme 28, reaction E). ${ }^{14}$ Under $\mathrm{N}_{2}$ or $\mathrm{H}_{2}$ atmosphere, photolysis of this last complex led to the formation of $\left[\mathrm{Cr}(\mathrm{CO})_{5} \mathrm{X}_{2}\right](\mathrm{X}=\mathrm{N}$ or $\mathrm{H})$ or cis-[Cr$\left.(\mathrm{CO})_{4}\left(\boldsymbol{\eta}^{2}-\mathrm{C}_{3} \mathrm{H}_{6}\right) \mathrm{X}_{2}\right]$ by losing either $\mathrm{C}_{3} \mathrm{H}_{6}$ or $\mathrm{CO}$.

Similarly to chromium complexes, photochemical ligand substitution was described for tungsten complexes. As piperidinium chloride and dipiperidylmethane 129 were observed after reaction of $c i s$ - $\left[\mathbf{W}(\mathbf{C O})_{4}(\text { pip })_{2}\right]$ with piperidine in DCM, Kociecka et al. decided to investigate the direct photochemical reaction of 129 with $\left[\mathrm{W}(\mathrm{CO})_{6}\right]$ in hexanes (Scheme 29, reaction B). ${ }^{115}$ Interestingly, the diamine was found to be a good chelating ligand, and the resulting complex 130 was more stable than $\left[\mathbf{W}(\mathbf{C O})_{4}(\text { pip })_{2}\right]$. The new bidentate amine tungsten complex 130 was the first example of a metal being coordinated by a diamine ligand with nitrogen atoms separated by such a short distance.

Nucleophilic photochemical substitutions of $\left[\mathbf{M}(\mathbf{C O})_{6}\right]$ were also studied by using buckminsterfullerene $\left(\mathrm{C}_{60}\right)$. Indeed, sunlight-inducted photoreactions of $\left[\mathrm{M}(\mathrm{CO})_{6}\right](\mathrm{M}=\mathrm{Mo}$ and W) with $\mathrm{C}_{60}$ in hexane led to the formation of $\left[\mathbf{M}(\mathbf{C O})_{5}\left(\boldsymbol{\eta}^{2}-\right.\right.$ $\left.\left.\mathrm{C}_{60}\right)\right] 131$ in $2 \mathrm{~h}$. The complexes were recovered in $80-90 \%$ yield (Scheme 30). ${ }^{116}$

\section{Scheme 28. Photochemical Reaction of $\left[\mathrm{Cr}(\mathrm{CO})_{6}\right]$ Leading to the Formation of Different Chromium Complexes}

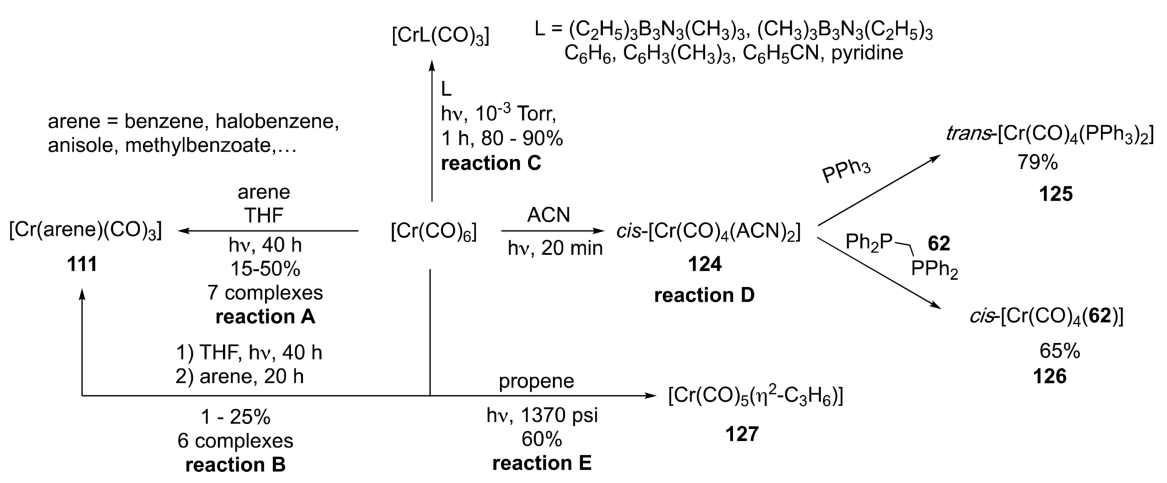


Scheme 30. Photochemical Synthesis of $\left[M(C O)_{5}\left(\eta^{2}-C_{60}\right)\right]$

\begin{tabular}{|c|c|c|}
\hline \multirow{3}{*}[\mathrm{M}(\mathrm{CO})_{6}]{} & $\mathrm{C}_{60}$ & \\
\hline & hexane & \\
\hline & $\begin{array}{l}\text { hv, } 2 \text { h } \\
80-90 \%\end{array}$ & 131 \\
\hline & $M=W, M o$ & \\
\hline
\end{tabular}

The photoreaction of $\left[\mathrm{Mo}(\mathbf{C O})_{6}\right]$ with $\mathrm{P}(n-\mathrm{Bu})_{3}$ was studied in 1980 by Post et al. and led to the formation of complexes bearing various substitution configurations: [Mo$\left.(\mathrm{CO})_{5}\left(\mathrm{P}(n-\mathrm{Bu})_{3}\right)\right] \quad 132$, cis- and trans $-\left[\mathrm{Mo}(\mathrm{CO})_{4}(\mathrm{P}(n-\right.$ $\left.\left.\mathrm{Bu})_{3}\right)_{2}\right] 133$, and cis- and trans- $\left[\mathrm{Mo}(\mathrm{CO})_{3}\left(\mathrm{P}(n-\mathrm{Bu})_{3}\right)_{3}\right] 134$ (Scheme 31, reaction A). ${ }^{117}$ The reaction could be performed using a sun lamp or a mercury lamp, with reaction time reduced to only 1 or $4 \mathrm{~h}$, respectively. Interestingly, the study included the separation of the product mixture by chromatography and led to pure complexes.

In 1987, Manuta et al. described the formation of complex $135\left(\left[\mathrm{Mo}(\mathrm{CO})_{4}\left(2,2^{\prime}\right.\right.\right.$-bipy $\left.\left.)\right]\right)$ by photolysis of a solution containing $\left[\mathrm{Mo}(\mathrm{CO})_{6}\right]$ and 2,2'-bipyridine using a mercury lamp $(200 \mathrm{~W}){ }^{118}$ In only $30 \mathrm{~min}, 135$ was recovered in $80 \%$ yield (Scheme 31, reaction B). Further substitution could be achieved by photochemical irradiation of a solution of 135 containing the coordinating $\mathrm{PPh}_{3}$ ligand. These conditions led to the formation of $\left[\mathrm{Mo}(\mathrm{CO})_{3}\left(2,2^{\prime}\right.\right.$-bipy $\left.)\left(\mathrm{PPh}_{3}\right)\right] 136$ after only $5 \mathrm{~min}$ of reaction (Scheme 31 , reaction $\mathrm{C}$ ). In the same conditions as described previously, photolysis of $[\mathbf{M o}(\mathbf{C p})$ $\left.(\mathrm{CO})_{3}\right]$ in the presence of liquid propene led to the formation of $\left[\mathrm{Mo}(\mathrm{Cp})(\mathrm{CO})_{2}\left(\eta^{2}-\mathrm{C}_{3} \mathrm{H}_{6}\right)\right] .^{114}\left[\mathrm{Mo}\left(\mathrm{Cp}^{*}\right)(\mathrm{CO})_{2}\left(\eta^{2}-\right.\right.$ $\left.\left.\mathrm{C}_{3} \mathrm{H}_{6}\right)\right]\left(\mathrm{Cp}^{*}=\right.$ pentamethylcyclopentadienyl $)$ was synthesized by thermal activation and was more stable than the $C p$ derivative.

Recently, Sener et al. described the photosynthesis of $\left[\mathrm{M}(\mathrm{CO})_{5}(\mathrm{~L})\right] 138$ from $\left[\mathrm{M}(\mathrm{CO})_{6}\right]$ and imine ligands $137(\mathrm{~L}$ = 3-[4-ethyl(phenyl)imino] $[1 H$-indol-2-one $]$ or 3-[4-butyl(phenyl)imino][1H-indol-2-one $]$ and $\mathrm{M}=\mathrm{Mo}, \mathrm{Cr}$, and $\mathrm{W}$ ) (Scheme 32). ${ }^{119}$ Similarly, 138 was formed starting from $\left[\mathrm{Mn}(\mathrm{CO})_{3} \mathrm{Cp}\right]$ and imine ligands upon decoordination of the $\mathrm{Cp}$ ligand.

6.4. Continuous Flow Chemistry for Synthesis of Chromium Complexes

In conventional conditions, syntheses of arene-chromium complexes are reported at high temperature using the arene as the solvent and reacting for 1-4 days. In addition, the procedure was complicated by the sublimation of $\left[\mathrm{Cr}(\mathrm{CO})_{6}\right]$
Scheme 32. Photosynthesis of $\left[\mathrm{M}(\mathrm{CO})_{5}(\mathrm{~L})\right]$ Complexes

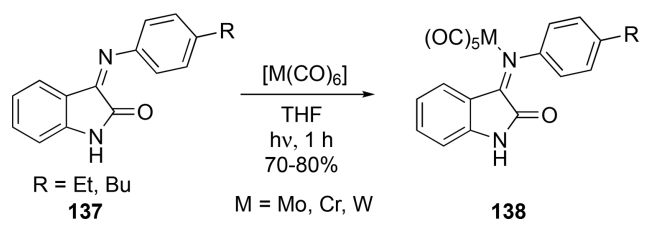

and the competitive decomposition of the arene chromium product due to long reaction times. These complexes could be synthesized more easily by an indirect route starting from naphthalene chromium tricarbonyl complex or with chromium complexes bearing three labile ligands. A continuous flow process was used in 2012 by Lee et al. to develop an efficient synthesis of the $\left[\mathrm{Cr}(\right.$ arene $\left.)(\mathbf{C O})_{3}\right]$ complex which avoided all usual synthetic problems. For example, $\left[\mathrm{Cr}\left(\mathrm{C}_{6} \mathbf{H}_{5} \mathbf{C H}_{3}\right)(\mathrm{CO})_{3}\right]$ 139 was synthesized starting directly from $\left[\mathrm{Cr}(\mathrm{CO})_{6}\right]$ and toluene in only $10 \mathrm{~min}$ (Scheme 33). ${ }^{120}$ This complex could be

Scheme 33. First Use of the Continuous Flow Process for the Synthesis of the Arene Chromium Tricarbonyl Complex

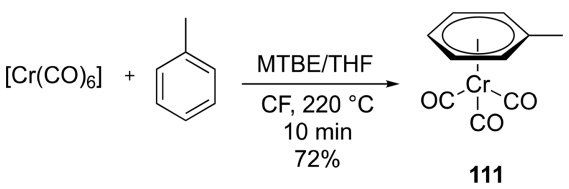

made on the gram scale and was recovered in $72 \%$ yield. Three other complexes were made with quantitative conversion by applying this procedure (cumene, chlorobenzene, and acetophenone). Unsurprisingly, the use of anisole or bromoacetophenone as substrates did not lead to the desired complexes, using neither this procedure nor the classical solution conditions. To the best of our knowledge, this is, today, the first and only synthesis of chromium complexes using a continuous flow process.

\section{GROUP 7}

\subsection{Mechanosynthesis of Rhenium Complexes}

Rhenium complexes have been thoroughly investigated for their fluorescence and applications in cell imaging. ${ }^{121}$ Interestingly, these rhenium species exhibited little or no intrinsic toxicity, while the ligand itself could be highly toxic. In 2014, the group of Frišcic developed the mechanosynthesis of families of complexes with the general formulas [Re-

Scheme 31. Photochemical Reaction of $\left[\mathrm{Mo}(\mathrm{CO})_{6}\right]$

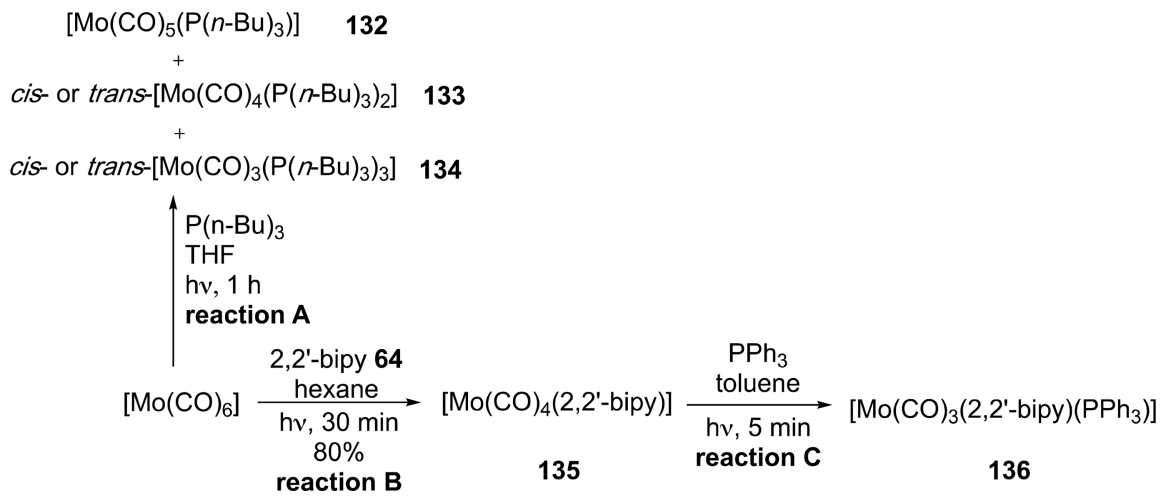


$\left.\mathrm{X}_{2}(\mathrm{CO})_{2} \mathrm{Cp}\right]$ and $\left[\operatorname{ReX}_{2}(\mathrm{CO})_{2} \mathrm{Cp}^{*}\right]^{122}$ Conventional syntheses involved complex starting materials, multistep reactions, photolysis, high pressures, and/or aggressive reagents such as halogens $\left(\mathrm{Br}_{2}\right.$, pyridinium perbromide) or acids (trifluoroacetic acid and $\mathrm{AlCl}_{3}$ ). To circumvent these issues, the authors described a simple and quick mechanosynthesis starting from metal halides, halogen salt, and oxone $\left(2 \mathrm{KHSO}_{5} \cdot \mathrm{KHSO}_{4}\right.$. $\mathrm{K}_{2} \mathrm{SO}_{4}$ ) in the absence of solvent. Two different isomers could be formed during the reaction: the diagonal (diag-) and the lateral (lat-) isomers 141 and 142 (Table 4). Using different

Table 4. Mechanosynthesis of Rhenium Complexes

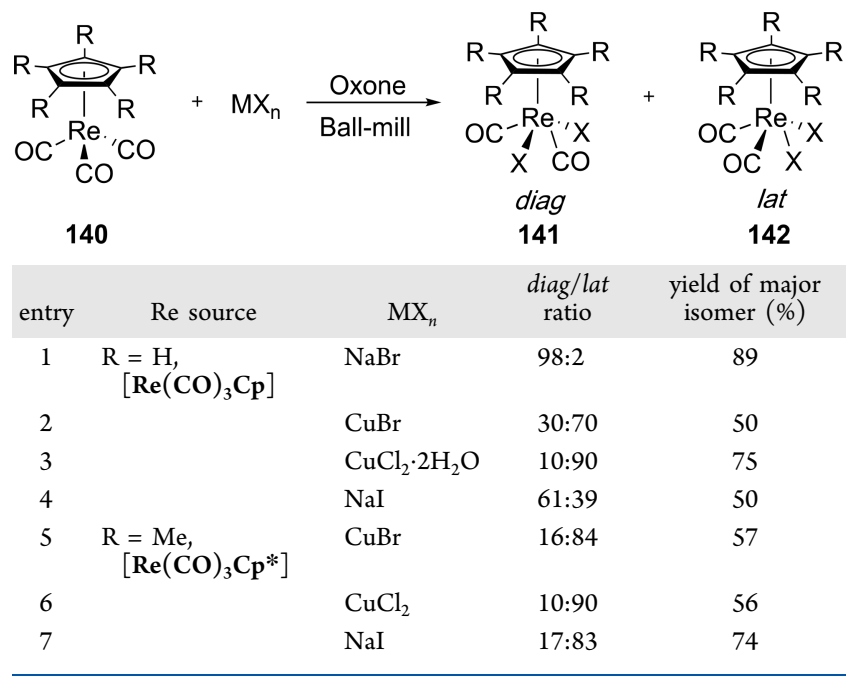

sources of bromide, an interesting change in selectivity was observed. Thus, milling $\mathrm{NaBr}$ with $\left[\operatorname{Re}(\mathbf{C O})_{3} \mathbf{C p}\right]$ for only 30 min at $30 \mathrm{~Hz}$ gave diag isomer 141 with a 98:2 selectivity (Table 4, entry 1). Contrastingly, milling the same rhenium complex with $\mathrm{CuBr}$ or $\mathrm{CuCl}_{2} \cdot 2 \mathrm{H}_{2} \mathrm{O}$ predominantly gave lat isomer 142 in 70:30 and 90:10 ratio, respectively (Table 4, entries 2 and 3). Interestingly, using $\mathrm{LiBr}$ as a source of bromide resulted in almost no selectivity. Yet, adding a few drops of water during milling gave only the diag isomer 141 . Similar syntheses were described with $\left[\operatorname{Re}(\mathbf{C O})_{3} \mathbf{C p}^{*}\right]$ as a substrate, giving corresponding complexes with good selectivity, yields higher than $50 \%$, and in only $30 \mathrm{~min}$ (Table 4, entries 5-7).

The same group also demonstrated the power of mechanochemistry for the synthesis of different rhenium complexes in the context of developing model radiopharmaceuticals containing the $f a c-\operatorname{Re}(\mathbf{C O})_{3}$ species. ${ }^{123}$ Five minutes of milling was necessary to obtain pentacarbonylrhenium halides $(\mathrm{Cl}, \mathrm{Br}, \mathrm{I})$ in $85-96 \%$ yield starting from $\left[\mathrm{Re}_{2}(\mathrm{CO})_{10}\right]$ (Scheme 34). Notably, pentacarbonylmanganese halide complexes 144 were synthesized using the same procedure in $28-88 \%$ yield. Furthermore, the use of mechanochemistry eliminated the need for elementary halogens, toxic solvents, or photolytic activation usually used for the synthesis of these compounds. Besides, complex 144 was transformed into a tribromide complex $\left[\operatorname{ReBr}_{3}(\mathrm{CO})_{3}\right] \cdot[\mathrm{TEA}]_{2} \quad 145$ by milling with an excess of tetraethylammonium bromide (TEAB) in a tungsten carbide milling jar placed in a vibratory ball mill operated at $30 \mathrm{~Hz}$. Ligand substitution using 1,10-phenanthroline was also possible in a ball mill. Finally, the efficiency of this approach was demonstrated in multicomponent reactions,
Scheme 34. Mechanosynthesis of Rhenium Complexes

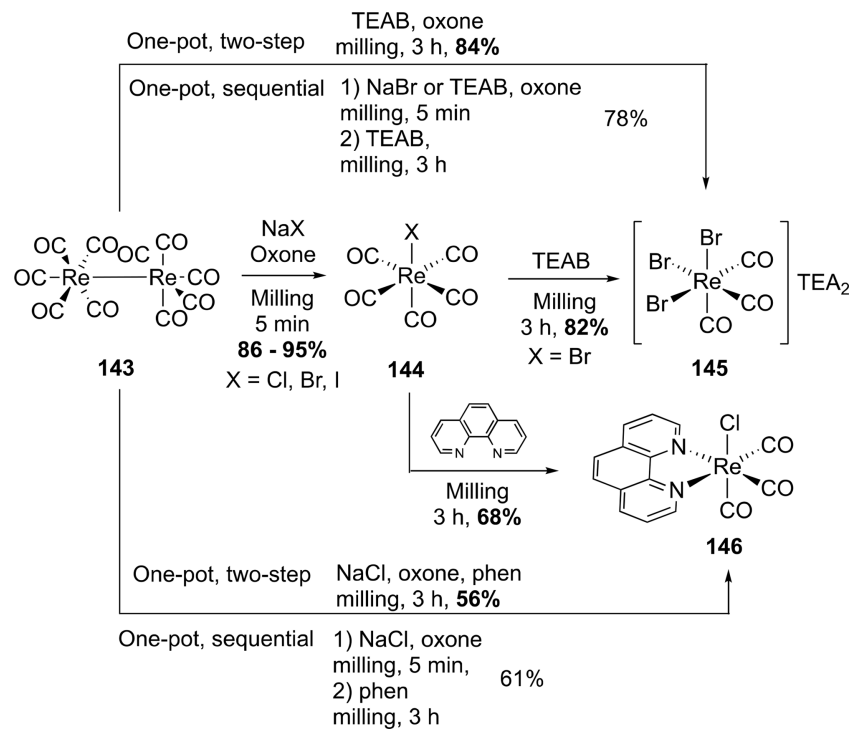

which could be performed in one pot, allowing the assembly of the complex or solution-sensitive organometallic species.

Similarly, rhenium complex 150 featuring $N, N, N^{\prime}, N^{\prime}$ tetramethylethylenediamine 149 (TMEDA) and a fluorine atom as ligands was mechanosynthesized in excellent yield (Scheme 35). Its formation could be performed sequentially, starting from $\operatorname{Re}_{2}(\mathrm{CO})_{10} 143$, through oxidative addition in the presence of sodium iodide and oxone, ligand exchange with a fluoride, and coordination of TMEDA. Alternatively, highly efficient one-pot procedures have been developed. Importantly, treatment of the reaction to form $\mathbf{1 5 0}$ had to be performed in a glovebox to avoid degradation of the moisturesensitive $\left[\operatorname{ReF}(\mathrm{CO})_{5}\right]$ intermediate 147. Indeed, in the presence of water, the pentacarbonyl rhenium fluoride complex was hydrolyzed into $\left[\operatorname{Re}(\mathrm{CO})_{3}\right]_{4}[\mathbf{O H}]_{4} \mathbf{1 4 8}$ (Scheme 35).

The superoxide anion has been associated with neurological disorders such as Parkinson's and Alzheimer's diseases. Among metal complexes capable of catalyzing dismutation of the superoxide anion, manganese complexes are effective and currently used for developing SOD (superoxide dismutase) mimics. Other biological properties of manganese complexes, such as the selective binding to quadruplex over duplex DNA (desoxyribonucleic acid), were also reported. ${ }^{6}$ Despite this, there is only one report of $\mathrm{Mn}$ complex mechanosynthesis in the literature. Milling manganese powder with ortho-quinone 151 and pyridine under atmospheric air led to the formation of the previously unknown mononuclear manganese complex $\left[\mathrm{Mn}(\mathrm{SQ})_{2}(\mathrm{Py})_{2}\right] 152$ (SQ = semiquinonate) using solid-state metal oxidation and direct coordination of the ligand formed in situ to the metal (Scheme 36). ${ }^{124}$ Similar conditions were used for the synthesis of numerous zinc complexes (see section 12.1).

\subsection{Microwave Irradiation for the Synthesis of Group 7 Metal Complexes}

In an early paper dealing with microwave syntheses, $\mathrm{KReO}_{4}$ and $\mathrm{PPh}_{3}$ were heated in ethanol/water $(10: 1)$ in a modified microwave oven $(250-350 \mathrm{~W}) .^{125}$ A round-bottom flask was placed in the microwave cavity with the water-cooled reflux condenser outside. This setup allows the solutions to heat quickly and to reflux safely without a buildup of pressure and the release of volatile solvents into the atmosphere (Figure 2). 
Scheme 35. Mechanosynthesis of the Fluoride-Containing Rhenium Complex

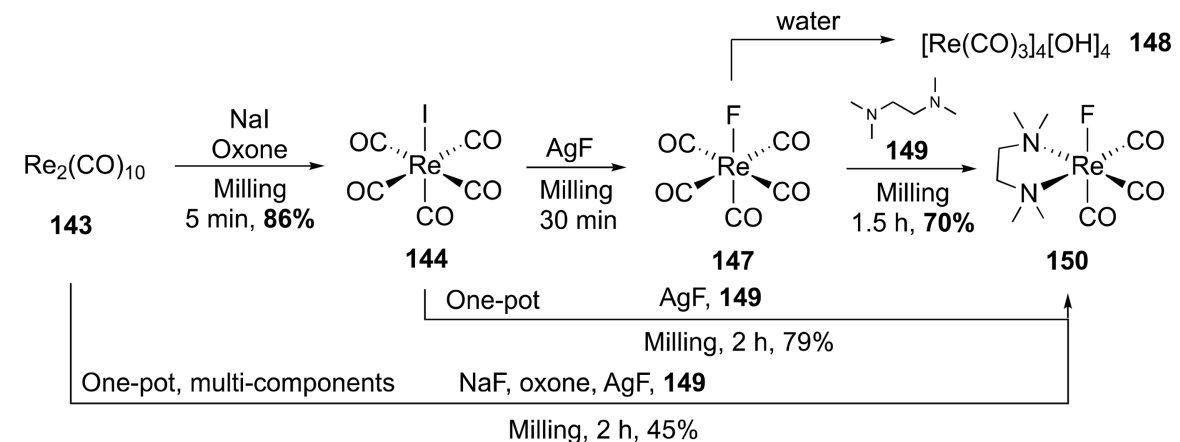

Scheme 36. Use of Manganese Powder for the Synthesis of the $\left[\mathrm{Mn}(\mathrm{SQ})_{2}(\mathrm{Py})_{2}\right]$ Complex
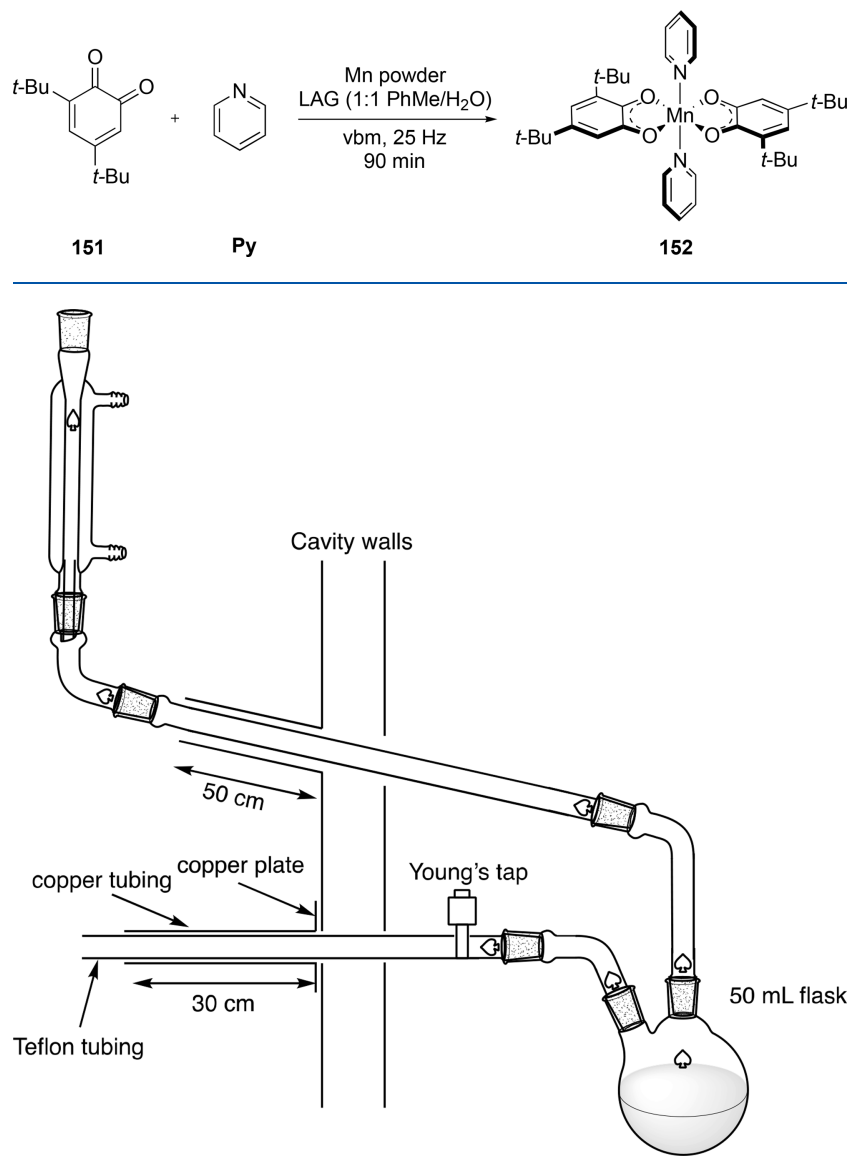

Figure 2. Modified microwave heating system.

The atmosphere in the flask could be controlled by a Teflon tube connected to an inert gas bottle. Using this homemade equipment, $\left[\operatorname{ReOCl}_{3}\left(\mathbf{P P h}_{3}\right)_{2}\right]$ was synthesized in $30 \mathrm{~min}$ of heating and isolated in $94 \%$ yield. Notably, in the literature $5 \mathrm{~h}$ was necessary to obtain full conversion. Even if the reaction time was considerably reduced via microwave irradiation, the temperature was difficult to control and could be $100{ }^{\circ} \mathrm{C}$ higher than in conventional reflux conditions.

In 2015, Reed et al. described the microwave synthesis of six dirhenium paddlewheel complexes 154 (Scheme 37). ${ }^{126}$ The reactions were performed under inert atmosphere and were heated in a Discover $S$ microwave reactor with an Explorer System $(2455 \mathrm{MHz})$. $\left[\mathbf{R e}_{2} \mathbf{C l}_{8}\right]\left[\mathbf{B u}_{4} \mathbf{N}\right]_{2} \mathbf{1 5 3}$ with a mixture of
Scheme 37. Synthesis of Dirhenium Paddlewheel Complexes

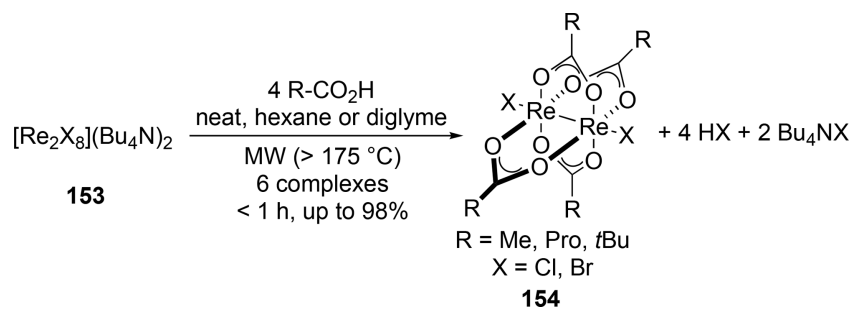

acid and anhydride was heated in neat conditions or with hexane or diglyme in a reaction vessel. In all cases, microwave synthesis allowed full conversion in significantly shorter time $(<1 \mathrm{~h})$ compared to the traditional reflux conditions ( $1 \mathrm{~h}$ to 4 days) and with higher yields. However, using butyric and pivalic acid resulted in increased reaction times and lowered yields. However, the method was still more efficient than a traditional reflux.

Starting from $\mathrm{MO}_{4}{ }^{-}\left(\mathrm{M}=\mathrm{Re},{ }^{99 \mathrm{~m}} \mathrm{Tc}\right.$, and $\left.{ }^{186 / 188} \mathrm{Re}\right)$, a rapid multistep microwave synthesis was developed for the preparation of molecularly targeted Tc-and Re-based imaging and therapy agents $\mathbf{1 5 5}$ (Scheme 38). ${ }^{127}$ Four steps developed

Scheme 38. Microwave Synthesis of Tc- and Re-Based Imaging and Therapy Agents

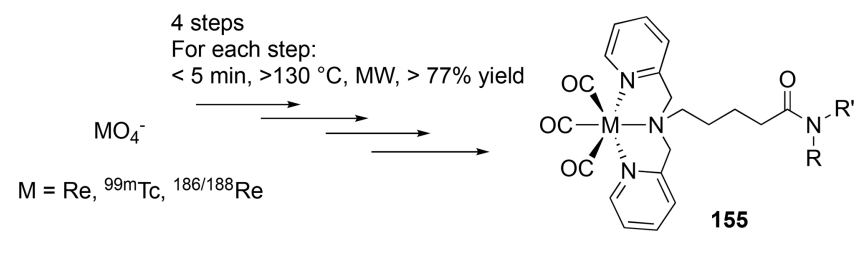

in a microwave oven using a Biotage Initiator Sixty instrument were necessary to form the desired molecules in only a few minutes and in high yield. First, $\mathrm{MO}_{4}{ }^{-}$was transformed into $\left[\mathrm{M}(\mathrm{CO})_{3}\left(\mathrm{H}_{2} \mathrm{O}\right)_{3}\right]^{+}$. The carbonyl precursor was then engaged with the pincer ligand that contained a terminal carboxyl group to form the coordinated complexes. The acid function was then transformed into an amide group in two steps to obtain the final complex 155. In order to create a useful multistep labeling procedure, rapid purification and workup were developed to minimize loss due to decay and to maximize the specific activity of the product. The entire synthetic procedure was completed in less than $30 \mathrm{~min}$, while $2 \mathrm{~h}$ was necessary using conventional heating.

A solution of $\left[\operatorname{Re}(\mathrm{CO})_{5} \mathrm{Br}\right] 144$ with 1 equiv of trispyrazolyl ligand 156 in toluene was irradiated for several cycles 
of 10 min using a CEM microwave system at $300 \mathrm{~W}$ to provide rhenium complexes 157 and 158 (Scheme 39). ${ }^{128}$ Reactions

\section{Scheme 39. Synthesis of Pyrazolylrhenium Complexes}
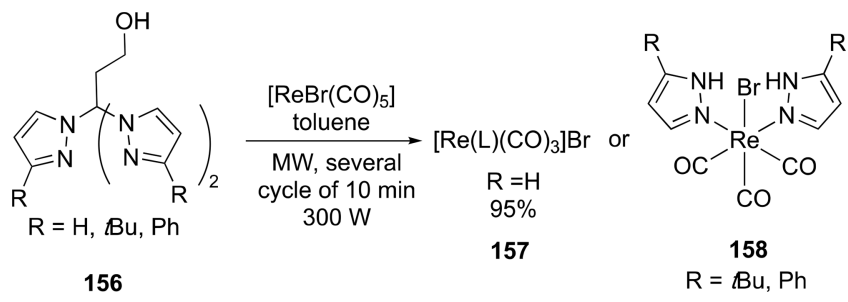

156

$$
\mathrm{R}=\mathrm{tBu}, \mathrm{Ph}
$$

were stopped when no change in the IR spectrum of the solution was observed. Different structures were obtained from the ligand screening. Indeed, the desired complex 157 featuring one tridentate ligand was obtained in $95 \%$ yield with $\mathrm{R}=\mathrm{H}$, but the unexpected $\left[\operatorname{ReBr}\left(\mathrm{Hpz}^{\mathrm{R}}\right)_{2}(\mathrm{CO})_{3}\right] 158$ $(\mathrm{pz}=$ pyrazole $)$ was identified with other substituents $(\mathrm{R}=t$ $\mathrm{Bu}$ or $\mathrm{Ph})$. A similar dehydroxy compound [Re$\left.\left((\mathrm{pz})_{3} \mathrm{CCH}_{2} \mathrm{CH}_{3}\right)(\mathrm{CO})_{3}\right] \mathrm{Br}$ was also isolated in $80 \%$ yield using the same strategy.

With the same goal of developing new radiopharmaceutical compounds, the group of Valliant developed a microwave synthesis of mono-, dialkyl, or aryl technetium and rhenium metallocarboranes (carboranes are polyhedral boron clusters). Rhenium source and carborane clusters were heated in ethanol using a Biotage Initiator Sixty Instrument at $200{ }^{\circ} \mathrm{C}$ for $15 \mathrm{~min}$ to provide the corresponding complexes 159 and 160 in good yields (Scheme 40). ${ }^{129}$ This microwave approach also

Scheme 40. Microwave Synthesis of Rhenium Metallocarboranes

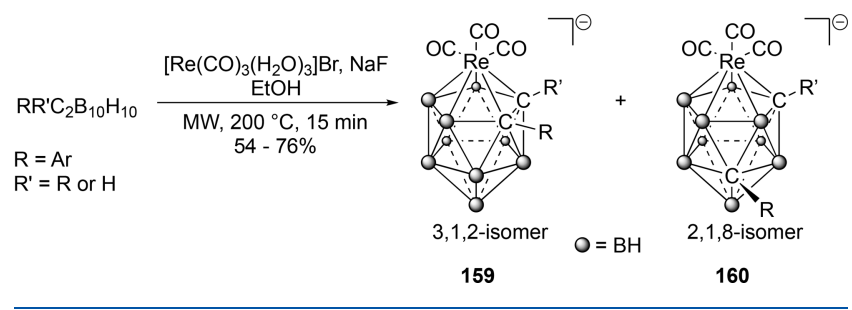

permitted the transformation of the 1,2 to 1,7 carborane cage isomer. Subsequently, this procedure was applied to six rhenium complexes and a technetium-99m analogue $(\mathrm{R}=$ $\left.\mathrm{PhOH}, \mathrm{R}^{\prime}=\mathrm{H}\right) .^{130}$ The technetium complex was isolated after $5 \mathrm{~min}$ of heating and reverse-phase purification, in a high radiochemical yield (84\%) and with a high effective specific activity. The affinities of these rhenium and technetium complexes for $\mathrm{ER} \alpha(\mathrm{ER}=$ estrogen receptor) and $\mathrm{ER} \beta$ were also determined (ER is an attractive target for molecular imaging due to its upregulation in various cancers).

In order to obtain active antibacterial molecules, the Jain group reported the synthesis of $\mathrm{Mn}$ (II) complexes with amidecontaining ligands, with the assumption that these compounds could be highly active since $\mathrm{Mn}$ (II) is a surrogate of $\mathrm{Mg}$ (II) and amide ligands are prevalent in biologically active molecules (Scheme 41). ${ }^{131}$ Both microwave and conventional heating procedures were described. Using microwave irradiation enabled the reduction of the reaction time from $2-3 \mathrm{~h}$ to only a few minutes and the increase of isolated yields. Complexes 162 were tested as antibacterials against E. coli
Scheme 41. Synthesis of Antibacterial Mn(II) Complexes
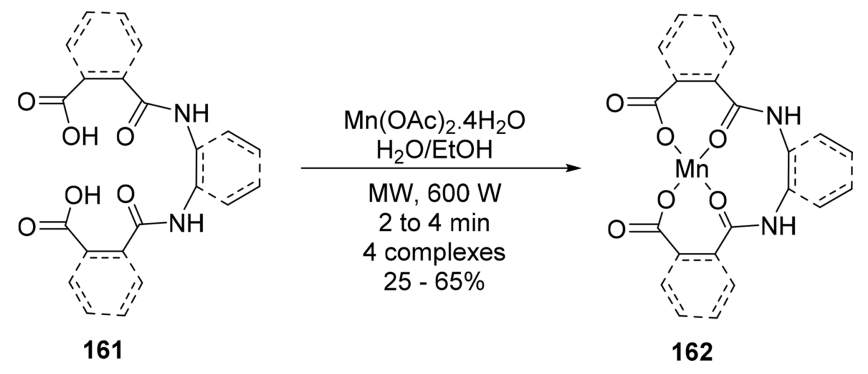

162

(Escherichia coli) and Staphylococcus aureus which gave promising activities.

$\mathrm{Xu}$ et al. adopted a similar approach for the formation of a manganese(II) complex featuring $\beta$-diketone ligand 163 via microwave irradiation (Scheme 42). ${ }^{132}$ Interestingly, complex 164 was synthesized by reacting two solid reactants, which were ground together prior to irradiation in the absence of a solvent. X-ray structure determination was realized, and 164 was tested for in vitro antibacterial activities against Grampositive (Bacillus subtilis, Staphylococcus aureus, Streptococcus faecalis) and Gram-negative strains (Pseudomonas aeruginosa, E. coli, and Enterobacter cloacae).

Several Schiff-base-containing $\mathrm{Mn}$ (II) complexes were synthesized using microwave irradiation (Table 5, see Figure 1 for the structure of ligands). $\left[\mathbf{M n}(79)_{2}\right]$ was obtained in $88 \%$ yield after $25 \mathrm{~s}$ of irradiation of $\mathrm{Mn}(\mathrm{OAc})_{2}$ and ligand 79 in an EtOH solution (Table 5, entry 1). ${ }^{133}$ To form complex $[\mathrm{Mn}(7 \mathbf{1})] \cdot \mathrm{H}_{2} \mathrm{O}$, the tridentate ligand $7 \mathbf{1}$ and $\mathrm{Mn}(\mathrm{OAc})_{2}$ were mixed together in a 1:1 ratio in a ball mill, and the reaction mixture was then irradiated in a microwave oven in EtOH for only 6 min (Table 5 , entry 2). ${ }^{134}$ Moreover, complex $\left[\mathrm{Mn}(\mathbf{8 7})_{2}\right]$ was isolated after $1 \mathrm{~min}$ of irradiation and in $83 \%$ yield (Table 5 , entry 3 ). ${ }^{135}$ In each case, microwave synthesis outperformed classical reflux conditions in terms of reaction times and yields. Similarly, complex $\left[\mathrm{Mn}_{7}(\mathrm{OH})_{6}(90)_{6}\right]\left[\mathrm{ClO}_{4}\right]_{2}$, which exhibits blue fluorescence emission at $458 \mathrm{~nm}$, could be obtained via microwave irradiation in $27 \%$ yield while heating an aqueous solution of $\mathrm{MnClO}_{4}$, 2-hydroxy-3-methoxybenzaldehyde, methylamine, and triethylamine at $80{ }^{\circ} \mathrm{C}$ (Table 5, entry 4). ${ }^{136}$ In comparison, the synthesis in an autoclave required $120 \mathrm{~h}$ of heating at $80{ }^{\circ} \mathrm{C}$ and cooling to room temperature over a period of $5 \mathrm{~h}\left(10{ }^{\circ} \mathrm{C} / \mathrm{h}\right)$ to afford the complex in $22 \%$ yield.

Microwave synthesis of various $\left[\mathbf{M n}(\boldsymbol{\eta}\right.$-arene $\left.)(\mathbf{C O})_{3}\right]\left[\mathbf{P F}_{6}\right]$ complexes 166 using a domestic microwave oven was described in 1997 (Scheme 43). ${ }^{57}$ In contrast with the classical conditions, aluminum powder was added to increase microwave absorption. This method enabled reduction of reaction times from several hours to only $3 \mathrm{~min}$. The yields were, however, significantly lower. Additionally, sterically hindered arenes reacted poorly and were partially dealkylated during the process. Reaction with fluorobenzene led to phenol complex formation due to the extreme sensitivity of the desired fluoride complex to hydrolysis.

The hexanuclear $\left[\mathrm{Mn}_{6} \mathrm{O}_{2}(\mathbf{s a o})_{6}\left(\mathrm{O}_{2} \mathrm{CH}\right)_{2}\left(\mathrm{CH}_{3} \mathrm{OH}\right)_{4}\right]$. $2 \mathrm{MeOH} 167$, which was found to be a single molecule magnet, was synthesized in $80 \%$ yield by microwave irradiation of a solution of $\left[\mathrm{Mn}\left(\mathrm{ClO}_{4}\right)_{2}\right] \cdot \mathbf{4} \mathbf{H}_{2} \mathrm{O}$, saoH $\mathbf{H}_{2}$ (salicylaldoxime), and $\mathrm{NaOMe}$ in $\mathrm{MeOH}$ for $5 \mathrm{~min}$ at $110^{\circ} \mathrm{C}$ (Figure 3). ${ }^{137}$ The complex could also be synthesized under ambient conditions, but the crystalline product did not appear immediately. In this 
Scheme 42. Microwave-Assisted Synthesis of a Mn(II)- $\beta$-Diketone Complex

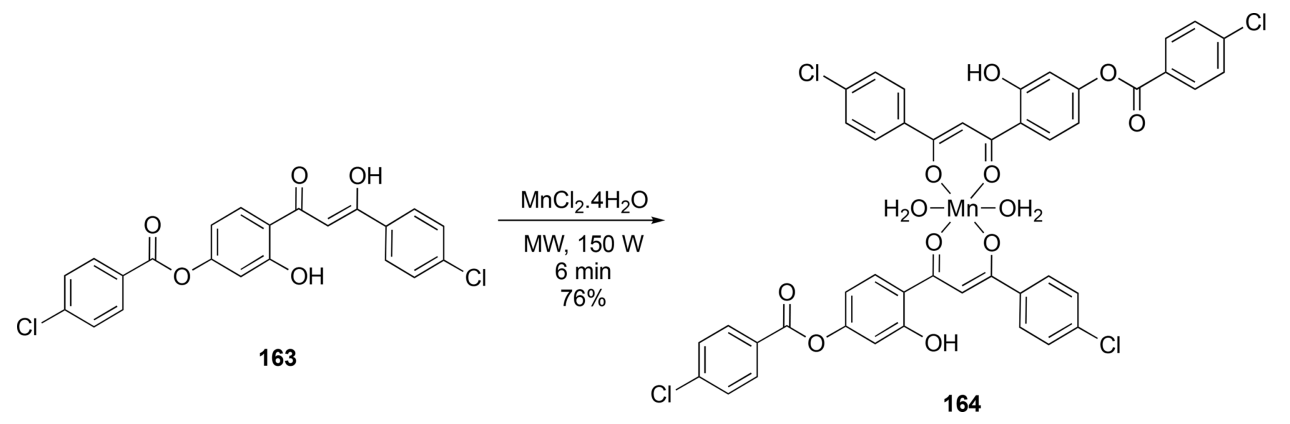

Table 5. Microwave Synthesis of Schiff Base-Mn(II) Complexes ${ }^{a}$

\begin{tabular}{|c|c|c|c|c|c|c|}
\hline \multirow[b]{2}{*}{ entry } & \multirow[b]{2}{*}{ manganese complex } & \multicolumn{2}{|c|}{ microwave synthesis } & \multicolumn{2}{|c|}{ conventional heating } & \multirow[b]{2}{*}{ ref } \\
\hline & & time (min) & yield (\%) & time (h) & yield $(\%)$ & \\
\hline 1 & {$\left[\mathrm{Mn}(79)_{2}\right]$} & 0.5 & 88 & 5.5 & 78 & 133 \\
\hline 2 & {$[\mathrm{Mn}(71)] \cdot \mathrm{H}_{2} \mathrm{O}$} & 6 & 82 & 10 & 64 & 134 \\
\hline 3 & {$\left[\mathrm{Mn}(87)_{2}\right]$} & 1 & 83 & 5 & 74 & 135 \\
\hline 4 & {$\left[\mathrm{Mn}_{7}(\mathrm{OH})_{6}(90)_{6}\right]\left[\mathrm{ClO}_{4}\right]_{2}$} & 55 & 27 & 125 & 22 & 136 \\
\hline
\end{tabular}

Scheme 43. Microwave Synthesis of Arene-Manganese Complexes

\begin{tabular}{|c|c|c|c|}
\hline & & $\begin{array}{c}\text { Al powder } \\
\mathrm{AlCl}_{3} \\
\text { 1,2,4-Trichlorobenzene }\end{array}$ & \\
\hline & $\begin{array}{c}+\operatorname{MnBr}(\mathrm{CO})_{5} \\
165\end{array}$ & $\begin{array}{c}M W, 3 \text { min } \\
36-56 \%\end{array}$ & $\begin{array}{c}{\left[\mathrm{Mn}(\eta \text {-arene })(\mathrm{CO})_{3}\right][\mathrm{PF}} \\
5 \text { complexes } \\
166\end{array}$ \\
\hline
\end{tabular}

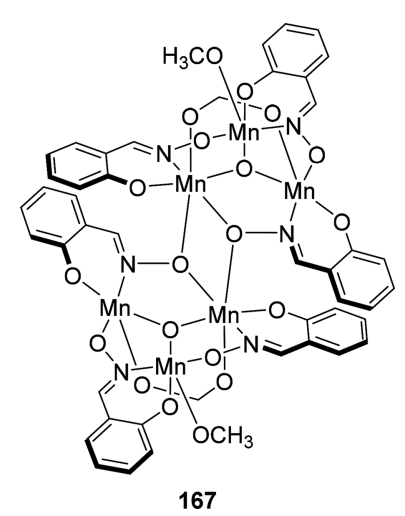

Figure 3. Structure of a single-molecule magnet synthesized by microwave irradiation.

case, the maximum yield (30\%) was achieved only after $60 \mathrm{~min}$ of reaction and 5 days of crystallization. Hence, microwave irradiation facilitated a highly efficient alternative.

7.3. Photochemical Synthesis of Manganese and Rhenium Complexes

Photochemistry was also used in manganese chemistry to promote ligand exchanges. $\left[\mathrm{Mn}\left(\mathrm{IEt}_{2} \mathbf{M e}_{2}\right)(\mathrm{CO})_{2} \mathrm{Cp}^{\prime}\right] \mathbf{1 7 0}$ ( $\mathrm{IEt}_{2} \mathrm{Me}_{2}=1,3$-diethyl-4,5-dimethylimidazol-2-ylidene, $\mathrm{Cp}^{\prime}=$ methylcyclopentadienyl) was isolated in $37 \%$ yield upon irradiation $(\lambda>285 \mathrm{~nm})$ of $\left[\mathrm{Mn}(\mathrm{CO})_{3} \mathrm{Cp}^{\prime}\right] 168$ in the presence of the free $\mathrm{N}$-heterocyclic carbene $169\left(\mathrm{IEt}_{2} \mathrm{Me}_{2}\right)$ in hexane at room temperature. ${ }^{138}$ Photolysis of this complex resulted only in one CO loss to afford $\left[\mathrm{Mn}\left(\mathrm{IEt}_{2} \mathbf{M e}_{2}\right)(\mathrm{CO})\right.$ $\left.\mathrm{Cp}^{\prime}\right]$ which was stabilized by a strong interaction with the
NHC ligand. In contrast, photolysis of $\left[\mathbf{M n}\left(\mathbf{P P h}_{3}\right)(\mathbf{C O})_{2} \mathbf{C p}^{\prime}\right]$ provided a mixture of $\left[\mathbf{M n}\left(\mathbf{P P h}_{3}\right)(\mathbf{C O}) \mathbf{C p}^{\prime}\right]$ and $[\mathbf{M n}$ $\left.(\mathrm{CO})_{2} \mathrm{Cp}^{\prime}\right]$. The same group has generalized this photochemical method to afford several $\left[\mathrm{Mn}(\mathrm{NHC})(\mathrm{CO})_{2} \mathrm{Cp}^{\prime}\right]$ complexes $\mathbf{1 7 0}$ in $37-51 \%$ yields (Scheme 44$).{ }^{139}$ If reactions

Scheme 44. Photochemistry of NHC-Mn Complexes
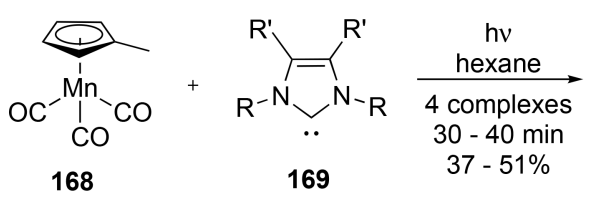

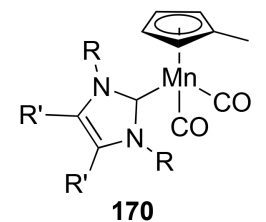

$\mathrm{R}=\mathrm{Alk}(\mathrm{Et}, \mathrm{iPr}), \mathrm{R}^{\prime}=\mathrm{Me}$

$\mathrm{R}=\operatorname{Ar}$ (Mes, Dipp), $\mathrm{R}^{\prime}=\mathrm{H}$

were complete in only $30 \mathrm{~min}$ with an alkyl substituent on the NHC, 10 more minutes is necessary with less reactive aryl NHC ligands. As described above, UV photolysis of these complexes with fast infrared detection allowed the detection of the monocarbonyl intermediates $\left[\mathbf{M n}(\mathrm{NHC})(\mathbf{C O}) \mathbf{C p}^{\prime}\right]$.

In 1990, the Sutton group described the UV photolysis of $\left[\operatorname{Re}(\mathrm{CO})_{3} \mathrm{Cp}^{*}\right] 140$ or $\left[\operatorname{Re}\left(\mathrm{N}_{2}\right)(\mathrm{CO})_{2} \mathrm{Cp}^{*}\right] 171$ in the presence of unsaturated organic ligands (alkenes, allenes, dienes, Scheme 45). ${ }^{140}$ Using this procedure, different $\left[\operatorname{Re}\left(\boldsymbol{\eta}^{2}\right.\right.$ alkene $\left.)(\mathrm{CO})_{2} \mathrm{Cp}^{*}\right],\left[\operatorname{Re}\left(\boldsymbol{\eta}^{2}\right.\right.$-allene $\left.)(\mathrm{CO})_{2} \mathrm{Cp}^{*}\right]$, and $\left[\operatorname{Re}\left(\boldsymbol{\eta}^{2}\right.\right.$ diene $\left.)(\mathrm{CO})_{2} \mathrm{Cp}^{*}\right]$ were synthesized within a very short time. Analogous $\left[\operatorname{Re}(\mathrm{L})\left(\boldsymbol{\eta}^{2}\right.\right.$-alkene $\left.)(\mathrm{CO}) \mathrm{Cp}^{*}\right]\left(\mathrm{L}=\mathrm{PMe}_{3}, \mathrm{H}\right)$ could also be photosynthesized starting from $[\operatorname{Re}(\mathrm{L})$ -

Scheme 45. Photochemical Synthesis of Re-Alkene Complexes

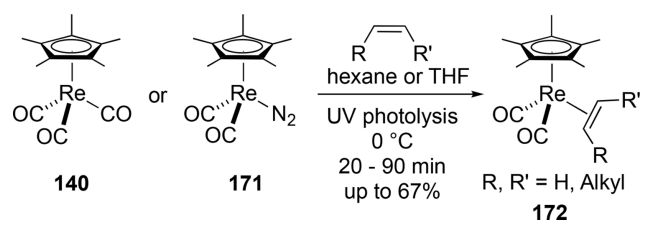


$\left.(\mathrm{CO})_{2} \mathrm{Cp}^{*}\right]$. When photolysis of rhenium alkene complexes 172 was prolonged, intramolecular $\mathrm{C}-\mathrm{H}$ activation occurred. This result established that rhenium-based organometallic compounds were capable of performing the carbon-hydrogen activation reaction.

\section{GROUP 8}

Iron complexes and in particular cyclopentadienyl iron complexes are highly active species that catalyze numerous reactions. ${ }^{141}$ They also play an important role in biology (activity against both estrogen-dependent and estrogenindependent breast cancer cells). ${ }^{6,142}$ Similarly, ruthenium complexes catalyze a wide number of reactions, the first of them being the well-known alkene metathesis reaction. ${ }^{143-145}$ Ruthenium complexes were also studied for their fluorescence and potential cell imaging applications, ${ }^{121}$ as well as anticancer agents to offer solutions to the limitations caused by platinum complexes (low efficacy against widespread tumors, intrinsic and acquired resistance of neoplasms, and severe side effects accompanying the therapy). Recently, the anticancer potential of osmium complexes was also explored. ${ }^{6,142}$ Due to the rising potential of these complexes, several research groups have reported the use of innovative techniques for their production and their subsequent transformation.

\subsection{Synthesis of Iron and Ruthenium Complexes Using} Ball Milling

Ferrocene complexes were mechanosynthesized for the first time in 1999 by the group of Makhaev. ${ }^{146}$ All experiments were carried out using a homemade eccentric vibratory ball mill. Potassium cyclopentadienide (KCp) and $\mathrm{FeCl}_{2}$ in a $3.4: 1$ ratio were subjected to mechanical milling for $15 \mathrm{~min}$ at a frequency of $12 \mathrm{~Hz}$ and an amplitude of $11 \mathrm{~mm}$, furnishing the corresponding complex 173 in $85 \%$ yield after sublimation (Scheme 46). Decreasing the amplitude from $11 \mathrm{~mm}$ to $3 \mathrm{~mm}$

\section{Scheme 46. Mechanosynthesis of Ferrocene}

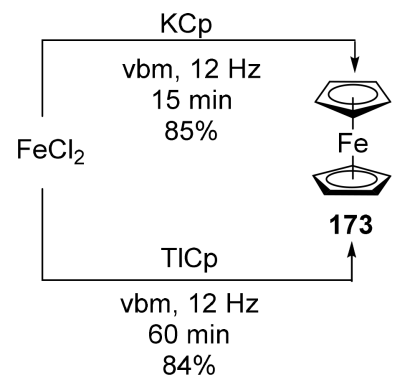

led to a very low conversion, with only traces of ferrocene 173 detected after $15 \mathrm{~min}$ of milling. It was also observed that stoichiometric amounts of KCp led to lower yields, which was attributed to the high oxygen and moisture sensitivity of KCp. To circumvent this, $\mathrm{TlCp}$ was used in a $\mathrm{TlCp} / \mathrm{FeCl}_{2}$ 1.8:1 ratio to form 173 in $84 \%$ yield after $60 \mathrm{~min}$ of grinding.
Interestingly, the solid-state reaction of ferrocene with $\mathrm{TlCl}_{2}$ was found to be reversible. Using the same method, $1,1^{\prime}$ dimethylferrocene was synthesized in $90 \%$ yield in $30 \mathrm{~min}$ of grinding starting from $\mathrm{NaMeCp}$ and $\mathrm{FeCl}_{2}$ in 2.1:1 ratio.

In 2002, the Braga group used the carboxylic-acid-containing ferrocene $\mathbf{1 7 4}$ to produce hydrogen-bonded organic-organometallic complexes via mechanosynthesis. ${ }^{147}$ Manual grinding of 174 and 1,4-diazabicyclo[2.2.2] octane or 1,4-phenylenediamine in a $1: 1$ ratio in an agate mortar and pestle led to the quantitative formation of the corresponding complexes (Scheme 47). The method was generalized to different solid bases (piperazine, trans-1,4-cyclohexanediamine, and guanidinium carbonate $\left.\left[\left(\mathrm{NH}_{2}\right)_{2} \mathrm{C}\right]_{2}\left[\mathrm{CO}_{3}\right]\right)$. The corresponding complexes were generated quantitatively as pure compounds, as verified by powder X-ray diffraction (PXRD) and comparison with data obtained by single-crystal X-ray diffraction. $^{148}$

In a similar way, hybrid organic-organometallic materials 177 and 178 could be formed via a solid-solid reaction of 1,1'-dipyridyl-ferrocene 176 with various organic diacids (fumaric, succinic, tridecanedioic, terephthalic, trimesic, and thiophene-2,5-dicarboxylic acids). ${ }^{149}$ Different structures ([Fe$\left.\left(\eta^{5}-\mathrm{C}_{5} \mathrm{H}_{4}-\mathrm{C}_{5} \mathrm{H}_{4} \mathrm{~N}\right)\left(\eta^{5}-\mathrm{C}_{5} \mathrm{H}_{4}-\mathrm{C}_{5} \mathrm{H}_{4} \mathrm{NH}\right)\right][\mathrm{A}-\mathrm{AH}]$ for 177 or $\left[\mathrm{Fe}\left(\eta^{5}-\mathrm{C}_{5} \mathrm{H}_{4}-\mathrm{C}_{5} \mathrm{H}_{4} \mathrm{NH}\right)_{2}\right][\mathrm{A}-\mathrm{A}]$ for 178$)$ could be obtained after mechanochemical treatment depending on the acid used (Scheme 48). Interestingly, mechanosynthesis of a maleic acid-iron complex yielded a different product from the one obtained under classical solution conditions.

Scheme 48. Synthesis of Hydrid Organic-Organometallic Materials

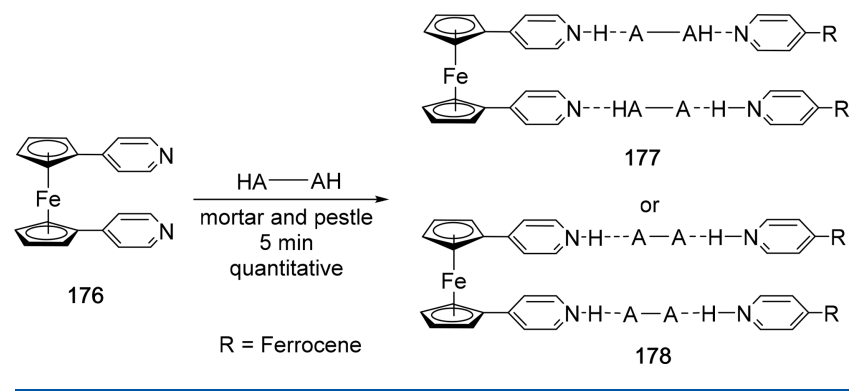

When linear alkyl diacids such as adipic, suberic, azelaic, and sebacic acids were utilized, new supramolecular macrocyclic adducts were synthesized (Figure 4). ${ }^{150}$ Thus, $\left[\mathbf{F e}\left(\boldsymbol{\eta}^{5}-\mathbf{C}_{5} \mathbf{H}_{4}\right.\right.$ $\left.\left.\mathrm{C}_{5} \mathrm{H}_{4} \mathbf{N}\right)_{2}\right] \cdot\left[\mathrm{HOOC}\left(\mathrm{CH}_{2}\right)_{n} \mathbf{C O O H}\right] 179(n=4,6,7,8)$ were obtained quantitatively by kneading powdered samples of the ferrocenyl complex and acid in a 1:1 ratio with a few drops of $\mathrm{MeOH}$. Surprisingly, when pimelic acid was used $(n=5)$, an isomeric open-chain structure was observed.

The Braga group also reported the mechanosynthesis of disubstituted pyridine/pyrimidine ferrocenyl complexes $\mathbf{1 8 2}$ using Suzuki-Miyaura coupling conditions (Scheme 49). ${ }^{151}$ The procedure required three grinding steps performed with a

\section{Scheme 47. Synthesis of a Hydrogen-Bonded Organic-Organoiron Complex}

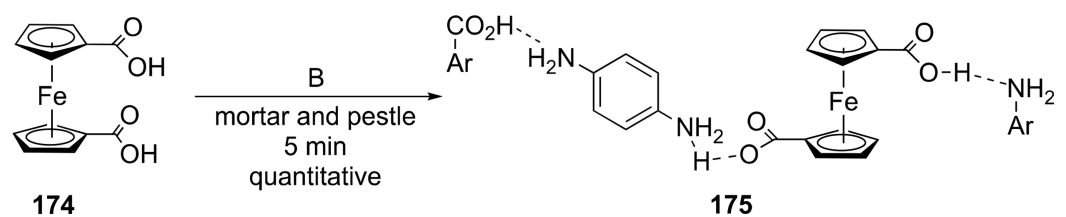




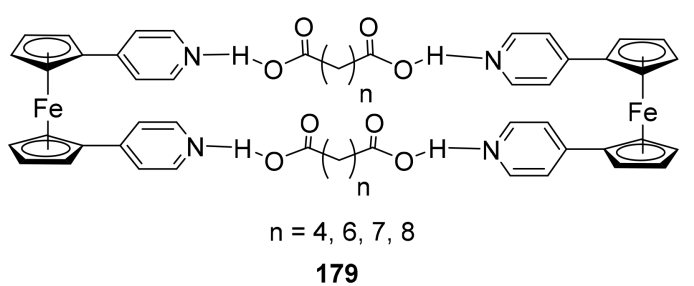

Figure 4. Supramolecular structure of macrocyclic adducts.

Scheme 49. Mechanosynthesis of Disubstituted Ferrocenyl Complexes

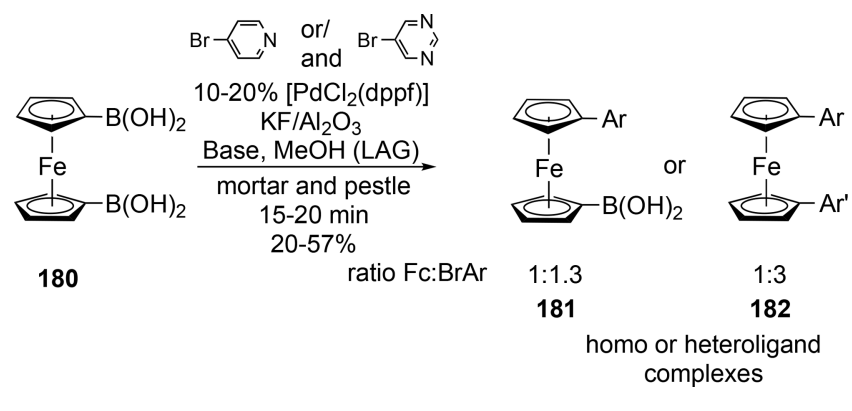

mortar and pestle: (i) grinding $\left[\mathrm{Fe}\left(\boldsymbol{\eta}^{5}-\mathrm{C}_{5} \mathrm{H}_{4} \mathrm{~B}(\mathrm{OH})_{2}\right)_{2}\right] \mathbf{1 8 0}$ and $\left[\mathbf{P d C l}_{2}(\mathbf{d p p f})\right]\left(\mathrm{dppf}=1,1^{\prime}\right.$-bis(diphenylphosphino)ferrocene, 67) in a $\mathrm{KF} / \mathrm{Al}_{2} \mathrm{O}_{3}$ mixture until complete homogenization, (ii) addition of the halogenated pyridine and grinding until homogenization, and (iii) addition of the base (KOH or KF) and further grinding for 15-20 min. For the last step, $\mathrm{MeOH}$ could be added to improve the efficiency of the synthesis. Since boronic acids are thermally stable and unreactive to both oxygen and water at ambient temperature and pressure, reactions could be carried out in air at room temperature. For this solvent-free Suzuki-Miyaura crosscoupling, the choice of $\left[\mathrm{PdCl}_{2}(\mathrm{dppf})\right]$ as the catalytic species was crucial since the use of $\left[\mathrm{Pd}\left(\mathrm{PPh}_{3}\right)_{4}\right]$ did not provide the desired complex. Interestingly, the reaction selectivity could be controlled in favor of the mono- or dicoupled products, 181 and 182, respectively, by changing the reagent stoichiometry.

The formation of imines has also been used to functionalize ferrocene derivatives. Unfortunately, some decomposition of ferrocenyl-imines is observed when they are produced by heating a solution of ferrocenyl-aldehyde and an aromatic amine. To avoid this, a simple, convenient, and milder approach was developed by grinding reactants at room temperature in a pyrex tube with a glass rod. ${ }^{152}$ Thus, mixing ferrocenyl-aldehyde 183 and aromatic amine in a 1:1 ratio provided the corresponding imine complex 184 in excellent yields and very short reaction times (Scheme 50). In the case of less reactive starting materials, gentle heating of the reaction mixture in a water bath $\left(50{ }^{\circ} \mathrm{C}\right)$ was required to obtain a good conversion. The procedure was also applied to $1,1^{\prime}$ ferrocenedicarboxaldehyde, ${ }^{153}$ dianiline, and diaminoalkanes $^{154}$ to afford the corresponding diimine compounds (186 and 185). The latter ferrocenyl complexes were used as ligands for the synthesis of rhodium complexes in solution.

Another modification of ferrocenylaldehyde was the formation of ferrocenyl-acrylonitriles $\mathbf{1 8 7}$ by mixing the aldehyde with 4-substituted phenylacetonitriles (Figure 5).
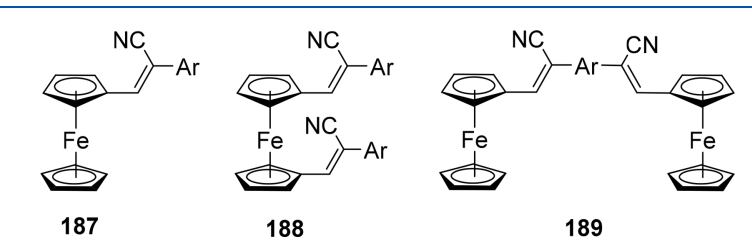

Figure 5. Acrylonitrile complexes obtained by mechanochemistry.

The corresponding products were obtained in excellent yields (89-99\%), except if the phenylacetonitrile beared a methoxy at the para position of the phenyl group. ${ }^{153}$ Synthesis of ferrocenyl-diacrylonitrile $\mathbf{1 8 8}$ and bis(ferrocenyl)-diacrylonitrile 189 could also be performed by mechanochemical treatment using a catalytic amount of piperidine during the grinding (Figure 5). ${ }^{155}$ However, this methodology also showed limitations as soon as phenylacetonitriles featuring an electron-donating group in the para position were used.

In 2013, Nieto et al. reported a facile one-pot route to prepare unexpected highly electron-poor 1-cyanocarbonylferrocene $191 .^{156}$ This procedure represented the first example describing the solid-state formation of a $-\mathrm{C}(\mathrm{O}) \mathrm{CN}$ bond without using an acyl chloride reactant. Simple manual grinding of cyanomethylferrocene 190 and silica gel in an agate mortar, in air, and at room temperature provided the oxidized complex in 53\% yield in less than $1 \mathrm{~h}$ (Scheme 51).

\section{Scheme 50. Highly Efficient Mechanosynthesis of Ferrocenyl-imines}

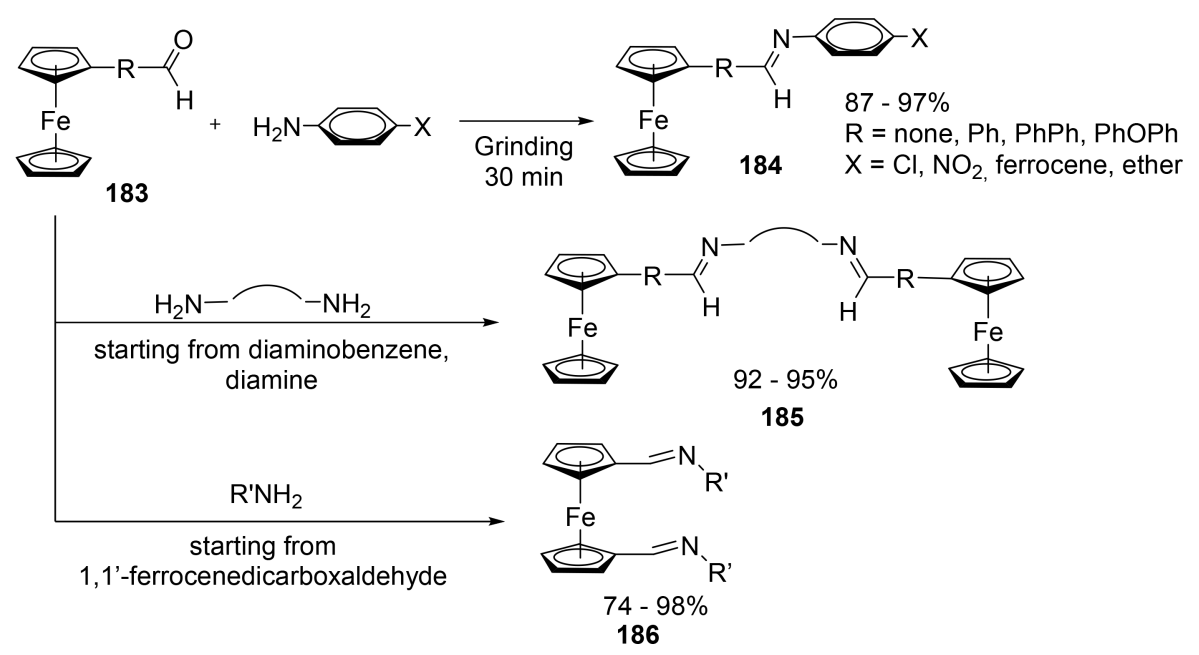


Scheme 51. Unexpected Mechanosynthesis of 1Cyanocarbonylferrocene

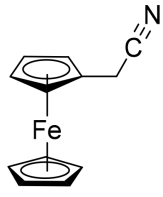

190

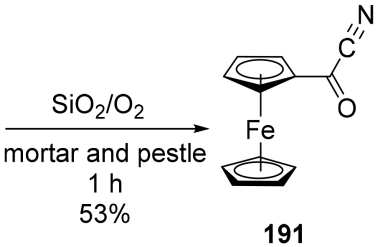

191
The slightly acidic surface of silica due to the presence of silanol groups was postulated to be responsible for the success of the reaction. The authors proposed a mechanism that was initiated by silica gel surface-mediated one-electron oxidation of the iron atom.

In 1993, Borisov et al. described the mechanosynthesis of iron(III) bisdicarbollyl complex 194 (Scheme 52). ${ }^{157}$ The reaction was performed in an eccentric vibratory ball mill. The steel reactor was charged with 15 steel balls $(12 \mathrm{~mm}$ in diameter), $\mathrm{FeCl}_{3}$, and $\mathrm{Tl}_{2} \mathrm{C}_{2} \mathrm{~B}_{9} \mathrm{H}_{11}$ 192. After $30 \mathrm{~min}$ of agitation at a frequency of $12 \mathrm{~Hz}$ and an $11 \mathrm{~mm}$ amplitude, the reaction mixture was recovered and treated with an excess of tetramethylammonium hydrogenosulfate to obtain 194 after filtration and recrystallization.

Hemin (195) is an organo-iron complex, which has also been synthesized by mechanochemistry. It has been used as a model to understand hemoglobin chemistry and as a starting reagent in the synthesis of pharmaceutical products for the treatment of anemia. Hemin has two potential sites that can react with a base, the two acidic groups, and the $\mathrm{Fe}^{\mathrm{III}}$ cation (Figure 6). They are important for the synthesis of complexes with pharmaceutical properties because of the modification of hydrogen bonding of this acidic residue with other $\mathrm{OH}$ groups. In 2002, Paneque et al. described different mechanochemical reactions of hemin with various fluorides. ${ }^{158}$ While $\mathrm{LiF}$ and $\mathrm{NaF}$ were found to be inert when milled with 195, KF, CsF, $\mathrm{NH}_{4} \mathrm{~F}$, and $\mathrm{AgF}$ (MF) reacted at both sites, leading to halide exchange on iron, as proved by the detection of MCl by XRD and the neutralization of the acidic groups to form $\mathrm{MHF}_{2}$. It was demonstrated that reactions first took place at the iron site and then at the peripheral acidic groups.

In 2015, Giri et al. described a subcomponent synthesis of three iron supramolecular complexes 198, 200, and 202 under solvent-free conditions (Scheme 53). ${ }^{159}$ Under selected ballmilling conditions, these three iron(II) complexes could be synthesized starting from a source of iron, 2-pyridinecarboxaldehyde 196, and three different amines (Scheme 53). Interestingly, a one-pot procedure was developed involving mixing of the iron source, aldehyde 196, and the three amines 197, 199, and 201, yielding the three complexes simultaneously. Notably, under mechanochemical treatment, complex 202 could be quantitatively converted into compound 198, which in turn could be converted into 200 as a consequence of the relative thermodynamic stability of these complexes.

Although the synthesis of many iron complexes was described by using mechanochemistry, to date only one

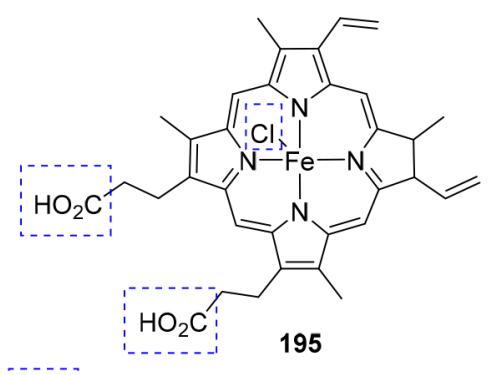

potential sites that can react with a base

Figure 6. Hemin structure.

publication reported the mechanosynthesis of a ruthenium complex. Thus, after manual grinding in a mortar and pestle of $\left[\mathbf{R u}\left(\mathrm{H}_{2} \text { biim }\right)_{3}\right]\left[\mathrm{PF}_{6}\right]_{2} \cdot 3 \mathrm{H}_{2} \mathrm{O} 203\left(\mathrm{H}_{2}\right.$ biim $=2,2^{\prime}$-biimidazole $)$ and $\mathrm{CH}_{3} \mathrm{CO}_{2} \mathrm{NH}_{4}$ with a few drops of $\mathrm{H}_{2} \mathrm{O}_{2}$ for $25 \mathrm{~min}$, complex 204 was recovered in $98 \%$ yield (Scheme 54). ${ }^{160}$ The reaction could also be performed at room temperature in solution, but the complex was isolated with a low yield of $30 \%$.

\subsection{Microwave Procedures}

The first synthesis of organo-iron complexes using microwave irradiation was reported by Dabirmanesh and Roberts in 1993. $\left[\mathrm{Fe}\left(\boldsymbol{\eta}-\mathrm{C}_{5} \mathrm{H}_{5}\right)(\eta\right.$-arene $\left.)\right] \mathrm{PF}_{6} 205$ and $\left[\mathrm{Fe}(\boldsymbol{\eta} \text {-arene })_{2}\right] \mathrm{PF}_{6} 206$ were synthesized from various arenes using a simple solid $\mathrm{CO}_{2}$ cooled apparatus in a conventional domestic microwave oven (Scheme 55). ${ }^{161}$ Syntheses of monoarene complexes 205 from ferrocene 173 (Scheme 55, reaction A) were facilitated by using microwave irradiation. Reaction times were decreased from hours to only a few minutes, and yields were improved. On the contrary, for the synthesis of complexes 206 bearing two arenes (Scheme 55, reaction B), yields were generally much lower than those obtained by conventional synthesis. Considerable rearrangements of the original arene by interand intramolecular migration of the methyl groups were observed. These rearrangements occurred prior to complexation and led to the formation of byproducts. In a detailed study, 1,2,4-trichlorobenzene (TCB) was identified as the solvent of choice, and the efficiency of metals as zinc or copper to replace the aluminum commonly used for the reaction was examined. The reaction scope was then extended to several arene compounds including sterically hindered ones. ${ }^{57,162} \mathrm{~A}$ mixture of ferrocene, arene, $\mathrm{AlCl}_{3}$, and $\mathrm{Al}$ powder in $\mathrm{TCB}$ was irradiated in an unmodified domestic microwave oven for 6 min to form corresponding heteroleptic complex 205, $\mathrm{HPF}_{6}$ being added during the workup procedure to afford the desired counteranion. Adding $\mathrm{W}(\mathrm{CO})_{4}\left(\mathrm{PPh}_{3}\right)_{2}$ to the reaction mixture provided $\left[\left[\mathrm{Fe}\left(\eta-\mathrm{C}_{6} \mathrm{H}_{5}\right)(\mathrm{Cp})\right]_{2} \mathrm{P}(\mathrm{Ph})\right]\left(\mathrm{PF}_{6}\right)_{2}$ and $\mathrm{POPh}_{3}$ produced $\left[\left[\mathrm{Fe}\left(\eta-\mathrm{C}_{6} \mathrm{H}_{5}\right)(\mathrm{Cp})\right] \mathrm{P}(\mathrm{O})(\mathrm{Ph})_{2}\right] \mathrm{PF}_{6}$. Interestingly, microwave-assisted decomplexation was attempted using graphite flakes or graphite powder and gave excellent results in a very short time (2 $\mathrm{min})$.

In 2006, Roberts reported the advantages of microwave heating applied to the production and modification of organoiron complexes, this time by performing $S_{N} A r$ reactions with

Scheme 52. Synthesis of the Iron(III) Bisdicarbollyl Complex

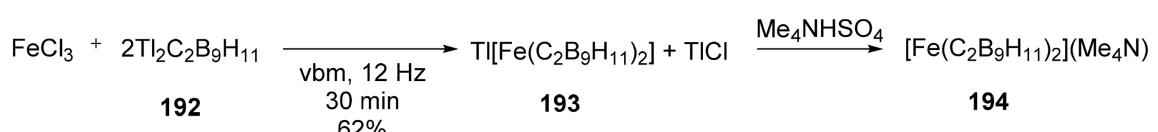


Scheme 53. Mechanosynthesis of Metallosupramolecular Iron(II) Complexes

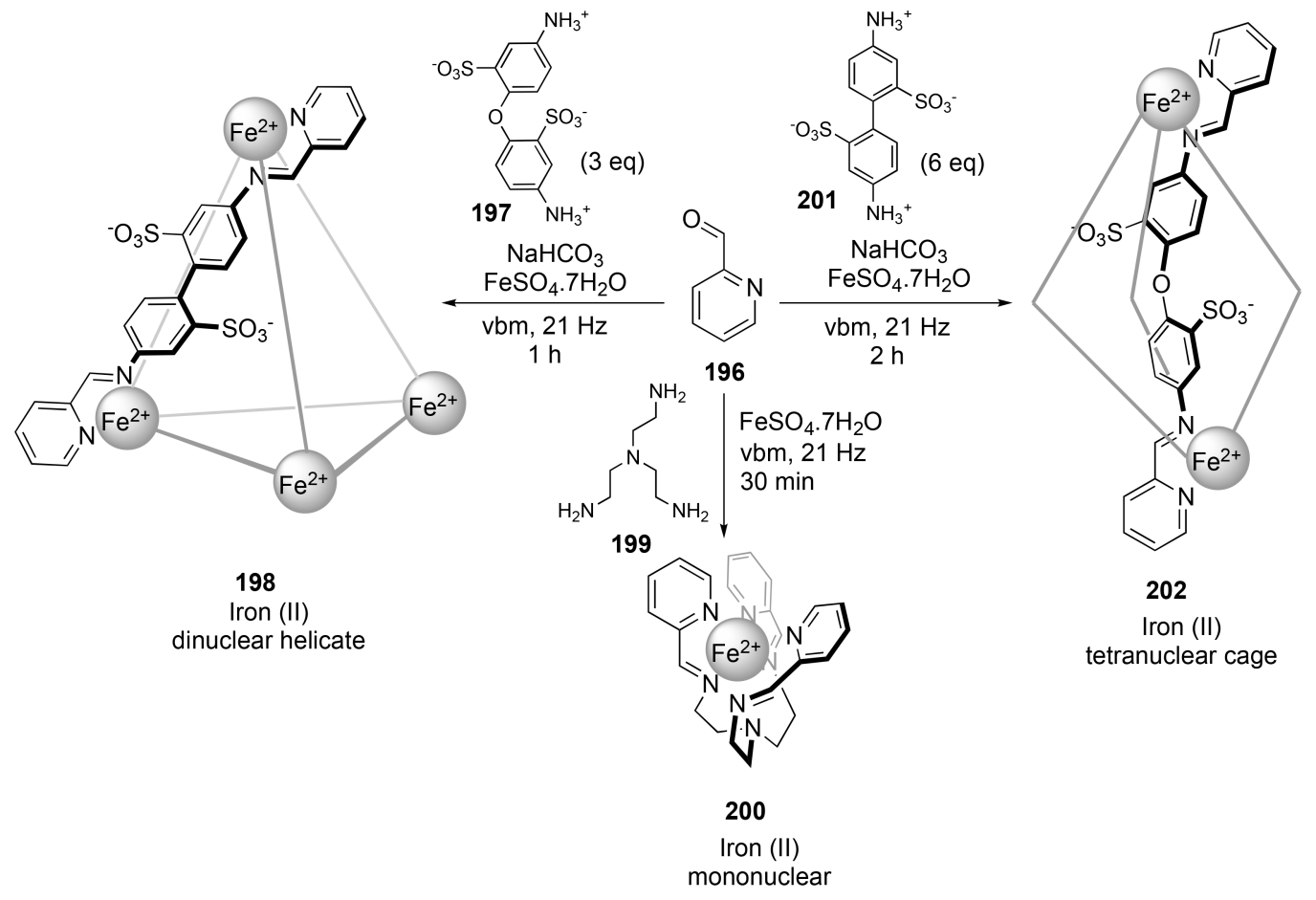

Scheme 54. Synthesis of the $\left[\mathrm{Ru}(\mathrm{Hbiim})_{3}\right]$ Complex

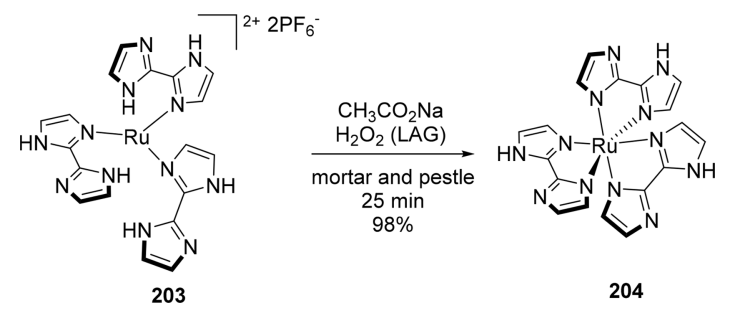

Scheme 55. Microwave Synthesis of Arene-Iron Complexes
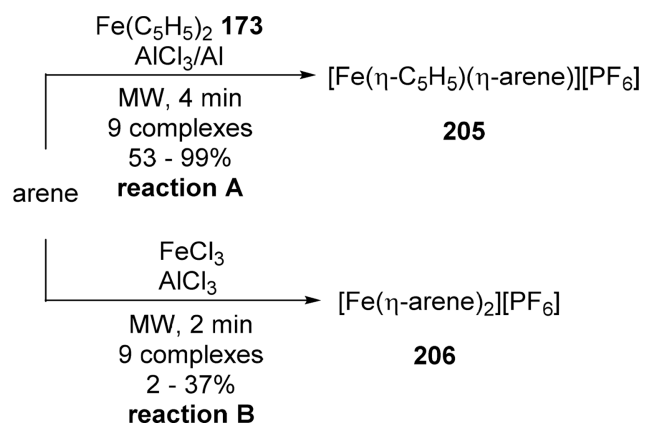

$\mathrm{N}$-containing nucleophiles. For instance, such $\mathrm{S}_{\mathrm{N}} \mathrm{Ar}$ reactions on complexes $205\left([\mathrm{Fe}(\mathrm{Cp})(\boldsymbol{\eta} \text {-arene })]^{+}\right)$performed by conventional heating can take place over several hours or, in some cases, over days. Using microwave irradiation accelerates the rate of these reactions leading to $S_{N} A r$ displacements in only 5 min (Scheme 56, reaction A). ${ }^{163}$ The technique was used to achieve a one-pot synthesis of an $\mathrm{N}$-arylated amino acid 210 (Scheme 55, reaction B). Afterward, decoordination of iron could be performed using $\mathrm{KOtBu}$ under microwave irradiation for $3 \mathrm{~min}$ to liberate unprotected amino acid $\mathbf{2 1 0}$.

In the same year, Roberts reported the use of microwave heating conditions to introduce oxygen substituents into iron
Scheme 56. Synthesis of Amino-Substituted ( $\eta^{6}$-Arene) $\left(\eta^{5}-\right.$ cyclopentadienyl)iron(II) Complexes

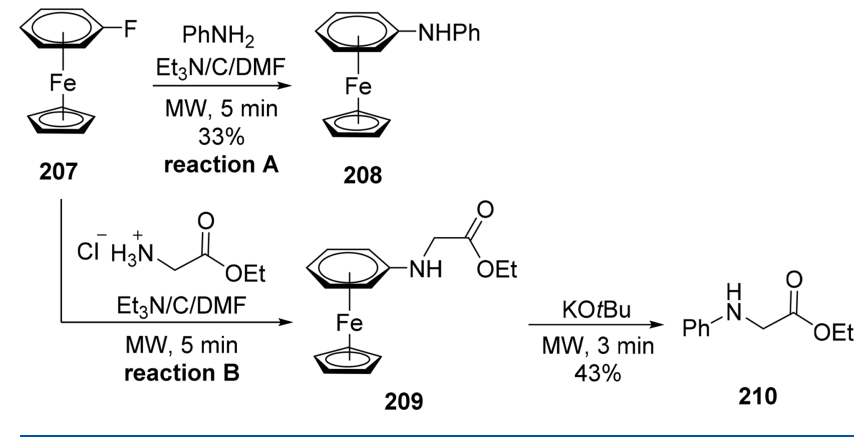

complex 205. ${ }^{164}$ Ligand exchange reactions (Scheme 55) performed on ferrocene with phenolic reactants using microwave irradiation resulted in the isolation of $[\mathrm{Fe}(\mathrm{Cp})$ $(\mathrm{ArOH})]$ complexes in very low yields, probably because of the strong complexation or reaction with $\mathrm{AlCl}_{3}$ that suppressed its Lewis acidity. Better results were obtained with phenyl-tbutylether and $O$-trimethylsilylphenol, resulting in the corresponding $[\mathrm{Fe}(\mathrm{Cp})(\mathbf{P h O H})]$ complex in $45 \%$ and $44 \%$ yield, respectively. When trimethylsilylated cathecol was used, the complex was obtained in $9 \%$ yield and was the first example in the literature of an ArFeCp complex containing two $\mathrm{OH}$ groups. Among the protecting groups tested, $t \mathrm{Bu}$ and $\mathrm{Me}_{3} \mathrm{Si}$ were the best ones because their steric hindrance probably inhibited complexation with $\mathrm{AlCl}_{3}$. Moreover, their deprotection proceeded concomitantly with the complexation reaction. Synthesis of a $[\mathrm{Fe}(\mathrm{Cp})(\mathrm{ArOR})]$ complex could also be performed under microwave irradiation by $S_{N} A r$ displacement of the halogen substituent of $[\mathbf{F e}(\boldsymbol{\eta}$ chlorobenzene $(\mathbf{C p})] \mathbf{P F}_{6}$ by phenol in the presence of $\mathrm{Et}_{3} \mathrm{~N}$. In this case, the reaction time was reduced to only 5 $\mathrm{min}$, while $12-15 \mathrm{~h}$ was required under classical conditions. 
In 2009, different organo-iron complexes were synthesized using a CEM Discover LabMate single-mode microwave instrument in minutes rather than days. ${ }^{165}$ First, ferrocene could be formed starting from a solution of dicyclopentadiene and $\mathrm{KO} t \mathrm{Bu}$ in diglyme irradiated at $200 \mathrm{~W}$ for $10 \mathrm{~min}$ that was subsequently added to a solution of $\mathrm{FeCl}_{2} \cdot 4 \mathrm{H}_{2} \mathrm{O}$ in DMSO ( $86 \%$ yield). Further modification of ferrocene into acetylferrocene could then be achieved using acetic anhydride and concentrated phosphoric acid as a catalyst placed under microwave conditions for $5 \mathrm{~min}$ ( $75 \%$ yield). The same authors showed that the challenging formation of the expensive $\left[\mathrm{Fe}(\mathrm{CO})_{2}\left(\mathrm{Cp}^{*}\right)\right]_{2}$ could be performed efficiently via microwave irradiation from $\left[\mathrm{Fe}(\mathrm{CO})_{5}\right]$ and pentamethylcyclopentadiene in DMF (200 W, 10 min, 88\% yield). $\left[\operatorname{FeCp}(\mathbf{C O})_{2} \mathrm{I}\right]$ 212 could also be synthesized from $\left[\mathrm{FeCp}(\mathrm{CO})_{2}\right]_{2} 211$ in $90 \%$ yield in only $10 \mathrm{~min}(150 \mathrm{~W})$ and transformed into $\left[\mathbf{F e C p}(\mathbf{C O})\left(\mathbf{P P h}_{3}\right) \mathbf{I}\right] 213$ using $\mathrm{PPh}_{3}$ (Scheme 57). A one-pot microwave synthesis starting from 211 was even more efficient.

Scheme 57. Microwave Synthesis of $\left[\operatorname{FeCp}(\mathrm{CO})\left(\mathrm{PPh}_{3}\right) \mathrm{I}\right]$

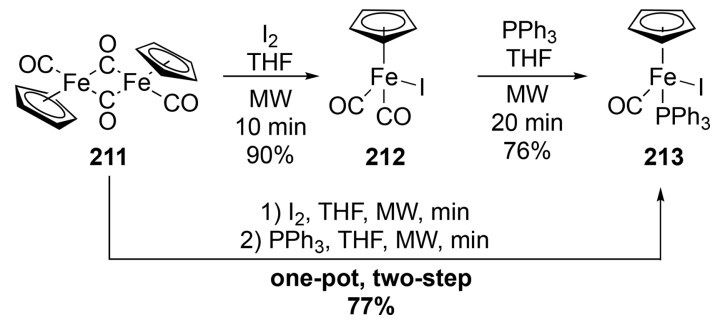

Additionally, $\left[\mathrm{FeCp}(\mathrm{CO})\left(\mathrm{PPh}_{3}\right)(\mathrm{COMe})\right] 215$ was synthesized in $86 \%$ yield starting from a solution of $[\mathrm{FeCp}$ $\left.(\mathrm{CO})_{2}(\mathrm{Me})\right] 214$ and triphenylphosphine in acetonitrile that was placed under microwave heating for $60 \mathrm{~min}$ (Scheme 58). ${ }^{165}$ Finally, four different phosphine complexes were

\section{Scheme 58. Photochemical Transformation of Iron} Complexes with Phosphine Ligand

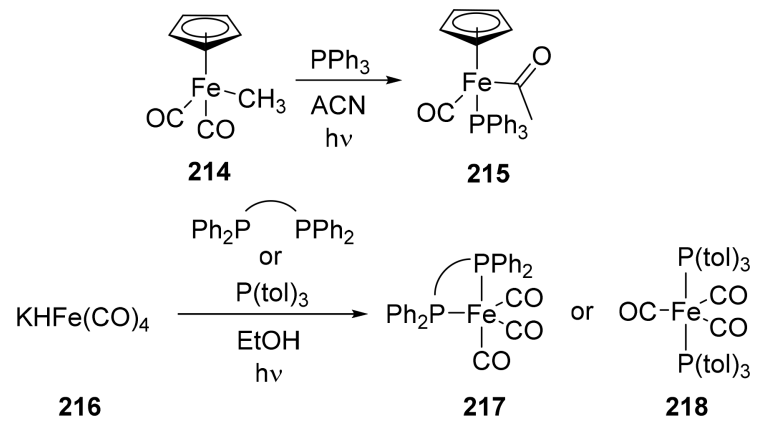

prepared via microwave irradiation of $\mathrm{KHFe}(\mathrm{CO})_{4}$ with the appropriate phosphine in an $\mathrm{EtOH}$ solution for $5 \mathrm{~min}$ at 150 W. Complexes 217 and 218 were recovered in $40-67 \%$ yield.

Two series of piano-stool iron(II) complexes bearing monodentate, bidentate diphosphine, or bidentate mixed P$\mathrm{N}$ ligands were prepared upon reaction with $\left[\mathrm{FeCp}(\mathrm{CO})_{2} \mathrm{I}\right]$ 219 or $[\mathrm{FeCp}($ naphthalene $)]\left[\mathrm{PF}_{6}\right] 223$ under microwave irradiation. ${ }^{166}$ If cationic species 221 were obtained by using different monophosphines (Scheme 59, reaction A), the neutral complex $\left[\mathbf{F e C p}\left(\mathbf{P P h}_{3}\right) \mathbf{I C O}\right]$ was obtained with triphenylphosphine in $90 \%$ yield. Lower yields were obtained for $\mathbf{2 2 1}$ due to the difficulty in isolating the pure product. The
Scheme 59. Microwaves Synthesis of $\left[\mathrm{FeCpL}_{3}\right][\mathrm{X}]$

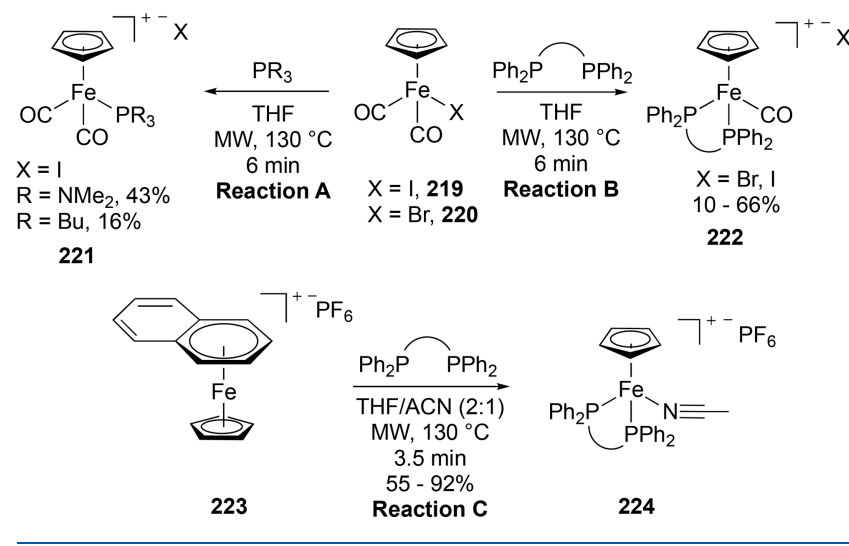

synthesis method was also applied to a diphosphine ligand producing complexes $\mathbf{2 2 2}$ with slightly better yields (Scheme 59 , reaction $\mathrm{B})$. Since the low lability of $\mathrm{CO}$ can be problematic for catalytic applications, $[\mathbf{F e C p}($ napth $)]\left[\mathbf{P F}_{6}\right]$ 223 was used as an alternative reactant to introduce a more labile acetonitrile ligand. After reaction with various diphosphines in the presence of acetonitrile under microwave irradiation, the corresponding complexes 224 were isolated in 55-92\% yield (Scheme 59, reaction C).

In 1994, Puciova et al. reported their difficulties in repeating the previously described syntheses of ferrocenyl-substituted heterocycles $\mathbf{2 2 7 - 2 3 3}$ by using conventional heating. With the purpose of improving these procedures, they performed these syntheses by using microwave irradiation (Scheme 60). ${ }^{167}$ Thus, ferrocenylthiophene $\mathbf{2 2 7}$ was prepared starting from 3chloro-3-ferrocenylacrylaldehyde $\mathbf{2 2 5}$ and thioglycolic acid in $\mathrm{Et}_{3} \mathrm{~N} / \mathrm{DMF}$. The reaction mixture was irradiated for $2 \mathrm{~min}$ to afford corresponding complex 227 in $87 \%$ yield. A similar procedure was applied successfully for the synthesis of ferrocenylfuran 229 and two different 1,1'-bis(2-thienyl)ferrocenes 228. Besides, heating 3-chloro-3-ferrocenylacrylaldehyde 225 under microwave irradiation with hydrazine in an $\mathrm{EtOH} / \mathrm{AcOH}$ (1:1) mixture produced the corresponding pyrazole 230. Despite this, when hydroxylamine, phenylhydrazine, and 2,4-dinitrophenylhydrazine were used instead of hydrazine, the reaction led only to product 232, which resulted from an acyclic condensation, as opposed to what was observed under classical conditions.

In the same year, Villemin et al. described microwaveassisted Claisen-Schmidt condensations of acetylferrocene 234 and ferrocene carboxaldehyde 236 with various electrophiles in neat conditions using $\mathrm{KOH}$ as a base and an ammonium salt as the catalyst (Aliquat 336). When benzaldehyde, furaldehyde, or 2-thienylcarboxaldehyde were used as the electrophiles, the reaction mixture did not need to be irradiated to obtain satisfactory results. However, with piperonal and paramethoxybenzaldehyde, irradiation at $140 \mathrm{~W}$ was necessary to obtain the corresponding complexes 235 in short time and good yields (Scheme 61, reaction A). ${ }^{168}$ When using ketones as the electrophiles (Scheme 61, reaction B), three out of the four ferrocene complexes were produced by using microwave irradiation due to the low reaction rate when the reaction was performed at room temperature.

In 2008, a similar microwave-assisted Claisen-Schmidt condensation was utilized to form 1,5-dioxo-3-ferrocenyl[5]ferrocenophane compounds 239 , which contain a bridging $\mathrm{C}_{5}$ chain between the two cyclopentadienyl rings of the same 
Scheme 60. Microwave Synthesis of Ferrocenyl-Substituted Heterocycles
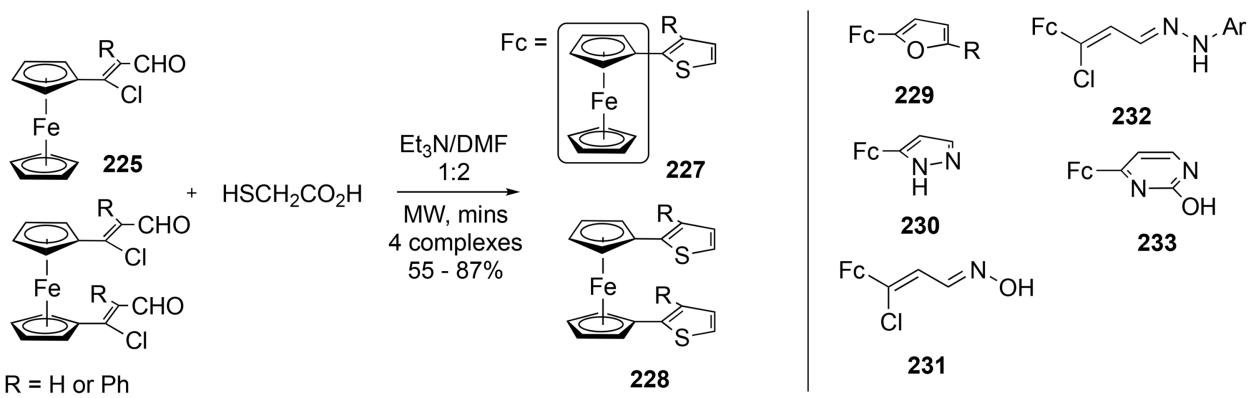

226

Scheme 61. Claisen-Schmidt Microwave Reactions

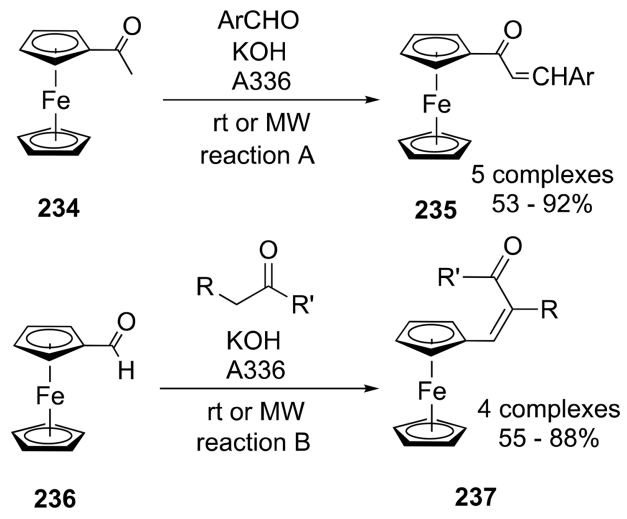

Scheme 62. Microwave Synthesis of 1,5-Dioxo-3-substituted [5]Ferrocenophanes

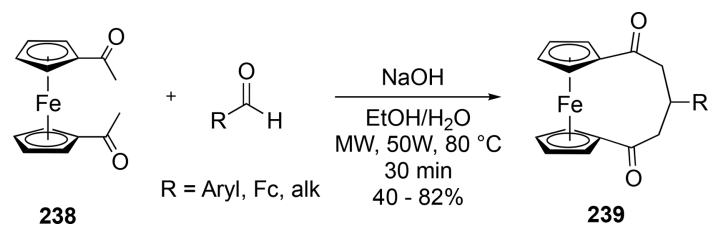

ferrocene unit and are compounds of interest for their electronic properties (Scheme 62). ${ }^{169}$ Complexes 239 were isolated in a short time and in moderate to good yields after submitting a solution of 1,10-diacetylferrocene 238 to microwave irradiation in the presence of an aldehyde and sodium hydroxide.

In 2017, Kulikov et al. described a microwave-assisted synthesis of ferrocenoyl hydrazones 241 starting from ferrocene carbohydrazide $\mathbf{2 4 0}$ and aldehydes in EtOH (Scheme 63). ${ }^{170}$ After a few seconds of heating, compounds 241 were recovered in very high yields (93-100\% yield) after a simple filtration of the resulting precipitate. Conventional heating led to higher reaction times and lower yields, presumably due to partial decomposition of either $\mathbf{2 4 0}$ or 241 under prolonged heating. This partial decomposition also led the authors to perform complicated chromatographic purification to isolate the desired products in satisfying purities.

Microwave irradiation also proved to be highly beneficial for the rearrangement of Dewar benzene-ferrocene complexes 242. While they did not rearrange upon heating to their melting point or to $150{ }^{\circ} \mathrm{C}$ in DMSO, heating at $180{ }^{\circ} \mathrm{C}$ resulted in their decomposition. Remarkably, their rearrangement toward the corresponding 1-benzene-ferrocene com- plexes 243 could be achieved when solubilized in THF and heated under microwave irradiation at $180{ }^{\circ} \mathrm{C}$ for $6 \mathrm{~h}$ (Scheme 64). ${ }^{171}$

Scheme 63. Synthesis of Ferrocenoyl Hydrazones under Microwave Irradiation

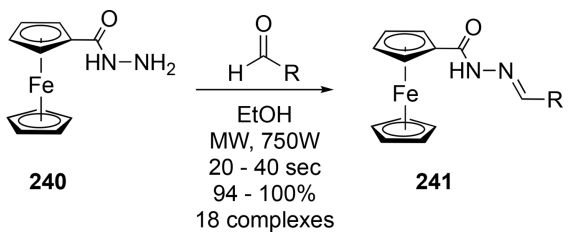

Scheme 64. Synthesis of Benzene-Ferrocene Complexes

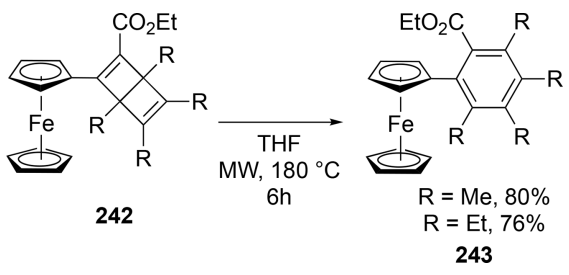

In contrast to synthesis by mechanochemistry, around 30 publications reported the synthesis of ruthenium complexes by using microwave activation. Different polypyridine-ruthenium complexes, which possess interesting photochemical and redox properties, were synthesized using microwaves (Scheme 65, reaction A) ${ }^{172}$ Starting from a stoichiometric ratio of the pyridine ligands and $\left[\mathrm{RuCl}_{3}\right] \cdot\left[\mathrm{H}_{2} \mathbf{O}\right]_{3}$ along with $\mathrm{Et}_{3} \mathrm{~N}$ in $\mathrm{MeOH}$, complexes were formed after irradiation in a domestic microwave oven at $650 \mathrm{~W}$ in a Teflon autoclave for 2 runs of $20 \mathrm{~s}$ (the autoclave was allowed to cool for $25 \mathrm{~min}$ before the second run). Addition of $\mathrm{KPF}_{6}$ in water led to the precipitation of the desired complexes 244 that could be isolated in good yields after filtration and washings.

The same general approach was published in 1994 for the synthesis of polypyridine-ruthenium complexes 247 via reflux under microwave irradiation in an open system (Scheme 65, reaction $\mathrm{B}) .{ }^{173}$ Starting from 10 different polypyridine ligands, products were recovered in good yields (57-95\%). Similarly, a series of new octahedral ruthenium(II)-polypyridine complexes were prepared via microwave irradiation to elucidate the effect of ligand structure on the redox potential of the complexes. Different ruthenium complexes of substituted bipyridine, benzimidazole, pyridine, quinoline, and phenanthroline were synthesized in $65-95 \%$ yields. Their $E_{\mathrm{L}}(\mathrm{L})$ parameter (derived from the oxidation potentials) was 
Scheme 65. Synthesis of Polypyridine-Ruthenium Complexes via Reflux under Microwave Irradiation

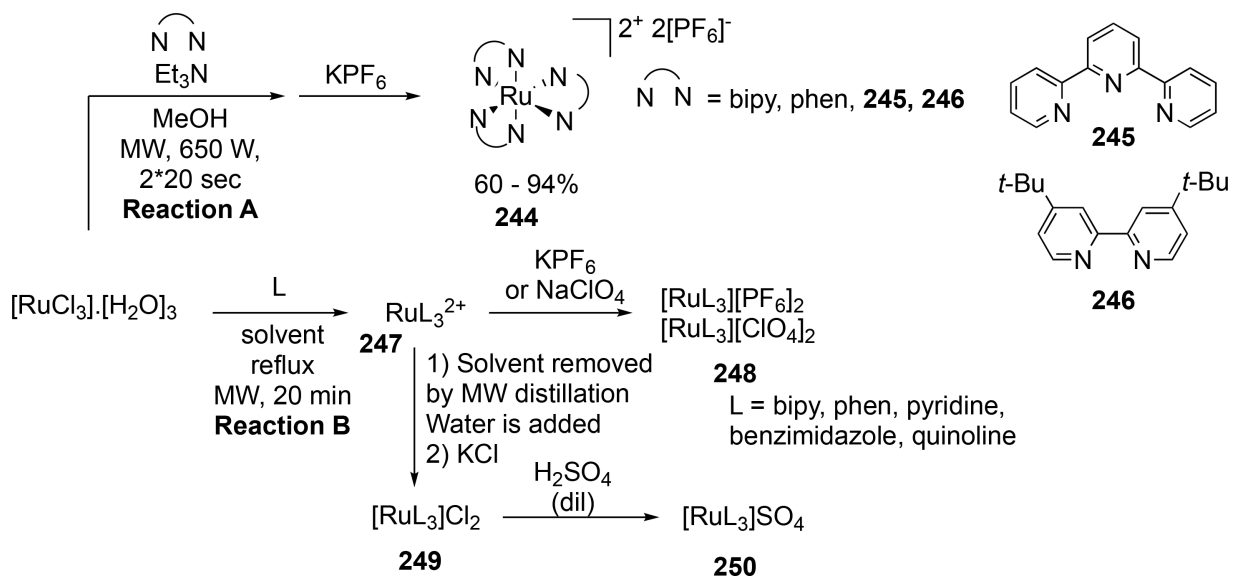

Scheme 66. Syntheses of Ru(II) Complexes by Microwave Heating
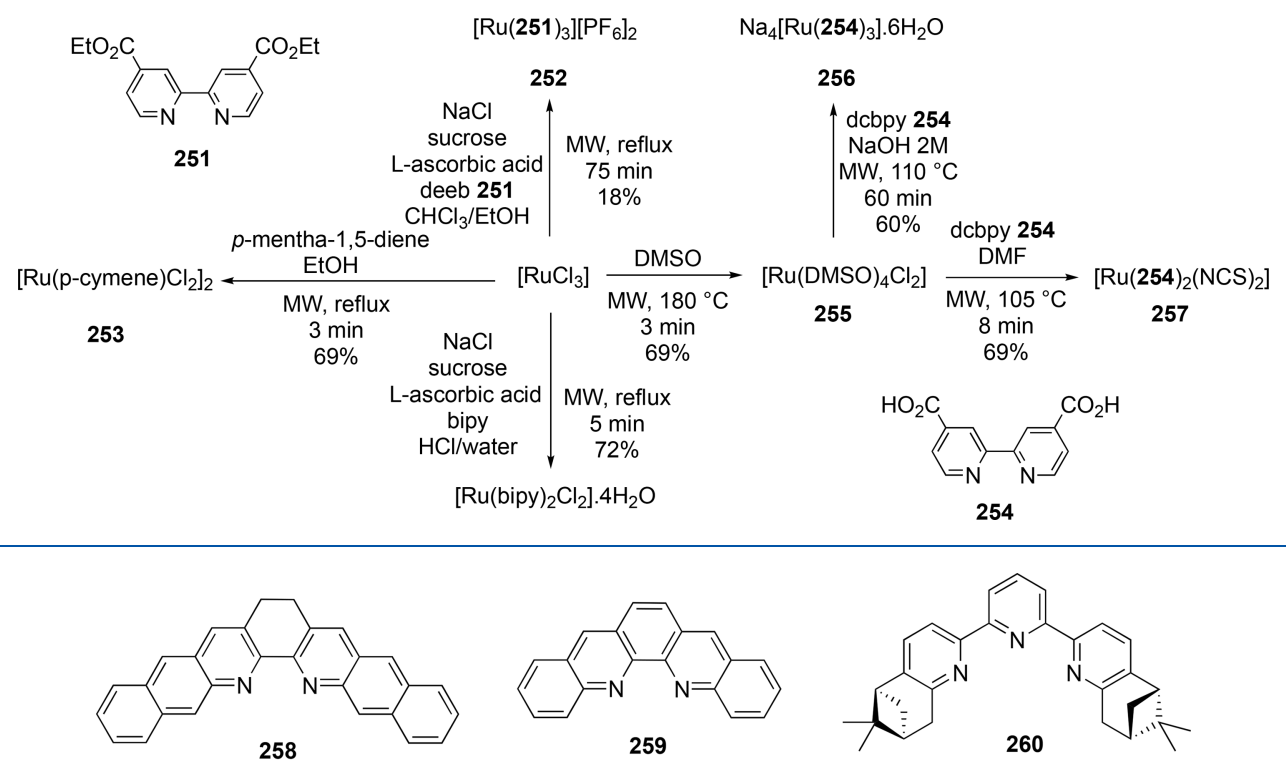

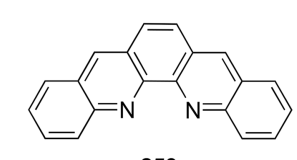

259

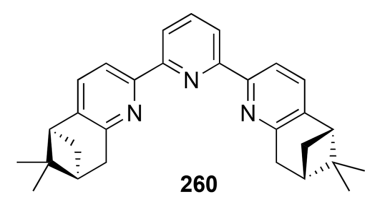

chiral terpyridyl-type ligand

Figure 7. Highly encumbered bipy ligands unreactive under conventional heating conditions.

correlated with the $\pi$-donor $/ \pi$-acceptor properties of the ligands. ${ }^{174}$ A comparable methodology was used for the synthesis of tris(4,7-diphenyl-1,10-phenanthroline)ruthenium(II) hexafluorophosphate from a dichloro( $p$ cymene)ruthenium(II) dimer, resulting in a complex with very promising electroluminescence behavior when operated in a solid-state light-emitting electrochemical cell. ${ }^{175}$

Recently, another publication reported a microwave-assisted synthesis of polypyridyl-ruthenium complexes. Starting from $\left[\mathbf{R u}(\mathrm{DMSO})_{4} \mathrm{Cl}_{2}\right]$, complexes $\left[\mathrm{Ru}(\mathrm{L})_{3}\right]\left[\mathrm{PF}_{6}\right]_{2}$ (L = bipy or phen) were obtained in $93-94 \%$ yields after 15 min of heating under microwave irradiation at $160{ }^{\circ} \mathrm{C} .{ }^{176}$ Starting from $\left[\mathrm{Ru}(\mathrm{L})_{2} \mathrm{Cl}_{2}\right]\left(\mathrm{L}=\right.$ bipy or phen), heteroleptic $\left[\mathbf{R u}(\mathbf{L})_{2}\left(\mathbf{L}^{\prime}\right)\right]$ $\left[\mathrm{PF}_{6}\right]_{2}$ (if $\mathrm{L}=$ bipy, $\mathrm{L}^{\prime}=$ phen; if $\mathrm{L}=$ phen, $\mathrm{L}^{\prime}=$ bipy) were obtained in $94-100 \%$ yield by using a similar procedure.

Microwave irradiation was also used for the synthesis of seven $\mathrm{Ru}(\mathrm{II})$ complexes to be employed in solar energy conversion and dye-sensitized solar cells (Scheme 66). ${ }^{177}$ All syntheses were performed using a CEM system in an open (atmospheric pressure) or closed vessel (pressurized conditions). Thus, $\left[\mathbf{R u}(\mathrm{DMSO})_{4} \mathbf{C l}_{2}\right] \mathbf{2 5 5}$ was synthesized in 3 min from a refluxing solution of $\mathrm{RuCl}_{3}$ in DMSO. This complex could then be transformed into $\mathrm{Na}_{4}\left[\mathrm{Ru}(\mathrm{dcb} p y)_{3}\right]$. 6 $\mathrm{H}_{2} \mathrm{O} 256$ (dcbpy = 4,4'-dicarboxy-2,2' -bipyridine 254) and $\left[\mathrm{Ru}(\mathrm{dcbpy})_{2}(\mathrm{NCS})_{2}\right] 257$ in $60 \%$ and $69 \%$ yields, respectively. Using the same equipment, $\left[\mathrm{Ru}(p \text {-cymene }) \mathrm{Cl}_{2}\right]_{2} 253$ was synthesized in $15 \mathrm{~min}$ in $65 \%$ yield from $\mathrm{RuCl}_{3}$ and $p$ mentha-1,4-diene. In the same publication, $\mathrm{RuCl}_{3}$ was also used to synthesize $\left[\mathrm{Ru}(\text { bipy })_{2} \mathrm{Cl}_{2}\right] \cdot\left(\mathrm{H}_{2} \mathrm{O}\right)_{4}$ by heating with 2,2'-bipyridine, $\mathrm{NaCl}$, sucrose, and L-ascorbic acid in an aqueous $\mathrm{HCl}$ solution. These reaction conditions provided one of the most difficult $\mathrm{Ru}(\mathrm{II})$ complexes, which possesses high quantum yield photoluminescence emission properties. [Ru$\left.(\text { deeb })_{3}\right]^{2+}$ complex 252 (deeb $=4,4^{\prime}$-diethylester-2,2' bipyridine 251) could indeed be produced in $18 \%$ yield in only $75 \mathrm{~min}$ of heating under microwave irradiaton. This contrasted greatly with the 7 days of conventional heating in solution required to isolate the complex in $15 \%$ yield. Furthermore, $\left[\mathbf{R u}(\text { bpy })_{2}(\mathbf{d c b p y})\right] \mathbf{C l}_{2} \cdot \mathbf{2} \mathbf{H}_{2} \mathbf{O}$ could be obtained in $93 \%$ yield by heating at $90{ }^{\circ} \mathrm{C}$ for $30 \mathrm{~min}$ in a solution of $\left[\mathbf{R u}(\text { bpy })_{2} \mathbf{C l}_{2}\right]$ and dcbpy $\mathbf{2 5 4}$ in a $\mathrm{AcOH} /$ water mixture. Interestingly, the majority of these complexes was recovered 
Scheme 67. Microwave Synthesis of Mixed Polypyridine Ruthenium Complexes

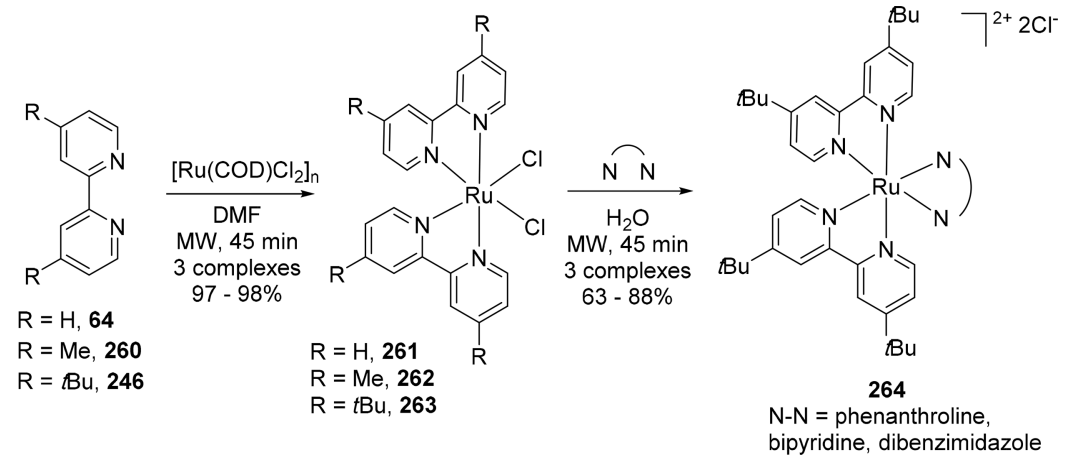

Scheme 68. Synthesis of $\left[\mathrm{Ru}(\mathrm{dcmb})_{3-n}(\mathrm{tbbpy})_{n}\right]\left[\mathrm{PF}_{6}\right]_{2}$ Using Microwave Irradiation

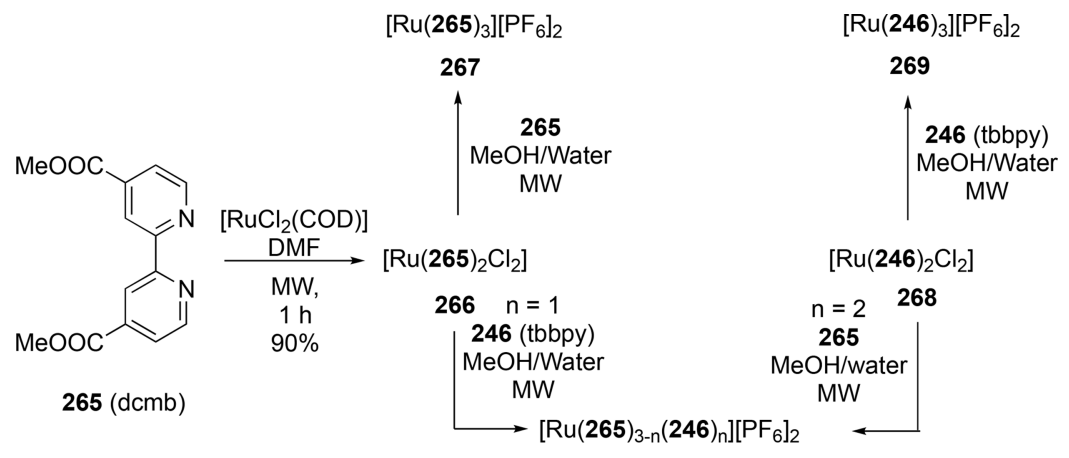

270

without column chromatography and was purified by simple work-ups.

Interestingly, microwave heating can assist the reactions of highly encumbered bipyridine ligands (3,3'-dimethylene-2,2' bibenzo[g]quinoline $\mathbf{2 5 8}$ and bisbenzo[2,3:9,8]-1,10-phenanthroline 259) to form their corresponding ruthenium complexes. Thus, the treatment of these bipyridines with $\left[\mathrm{RuCl}_{3}\right] \cdot\left[\mathrm{H}_{2} \mathrm{O}\right]_{3}$ or $\left[\mathrm{Ru}(\text { bipy })_{2} \mathrm{Cl}_{2}\right] \cdot\left[\mathrm{H}_{2} \mathbf{O}\right]_{2}$ in aqueous EtOH at reflux did not lead to the formation of the corresponding ruthenium complexes (Figure 7). However, running reactions in ethylene glycol for 15-30 min under microwave heating led to the formation of $\left[\mathbf{R u}(\mathbf{L})_{3}\right]\left[\mathbf{P F}_{6}\right]_{2}$ complexes in 15 and $19 \%$ yield. ${ }^{178}$ Better yields were obtained in the synthesis of $\left[\mathbf{R u}(\mathrm{L})\left(\text { bipy-d } \boldsymbol{d}_{8}\right)_{2}\right]\left[\mathrm{PF}_{6}\right]_{2}$ from cis- $\left[\mathrm{Ru}\left(\text { bipy- } \boldsymbol{d}_{8}\right)_{2} \mathbf{C l}_{2}\right] \cdot\left(\mathrm{H}_{2} \mathrm{O}\right)_{2}$ (41 and $44 \%$, respectively). In all cases, $\mathrm{NH}_{4} \mathrm{PF}_{6}$ was used during the workup procedure to obtain the desired counterion. Similarly, ruthenium complexes with annelated $2,2^{\prime}: 6^{\prime}, 2^{\prime \prime}$ terpyridine ligand ${ }^{179,180}$ and with enantiomerically pure chiral terpyridyl-type ligand $\mathbf{2 6 0}{ }^{181}$ were synthesized in a few minutes and in yields of up to $95 \%$. A range of homoleptic electron-withdrawing pyrrolidine-containing bipyridine and terpyridine ruthenium complexes have also been prepared using microwave-assisted heating. Complexes were obtained in high yields and very short reaction times $(3 \mathrm{~min})$, while classical thermal conditions failed. ${ }^{182}$

Bipyridine substitution of a COD ligand (COD = cycloocta1,5-diene) on the ruthenium complex $\left[\mathrm{Ru}(\mathrm{COD}) \mathrm{Cl}_{2}\right]_{n}$ was shown to be possible by microwave activation. The corresponding luminescent mixed polypyridine ruthenium complexes 261-263 were obtained in $45 \mathrm{~min}$ and in high yields (Scheme 67). ${ }^{183}$ Subsequent substitution of the chlorine atoms with different $\mathrm{N}, \mathrm{N}$-ligands (substituted phenanthroline-, bipyridine-, and dibenzimidazole-type ligands) was also possible by microwave heating in water. The use of microwaves considerably decreased the reaction time and provided the products in excellent yields and purities. Alternatively, $[\mathbf{R u}(\mathbf{R}$ bipy $)_{2} \mathrm{Cl}_{2}$ ] complexes 261-263 $(\mathrm{R}=\mathrm{H}, \mathrm{Me}$, tert-butyl) could be obtained in a few minutes and in quantitative yields by applying microwave heating to a solution of $\left[\mathrm{RuCl}_{3}\right] \cdot\left(\mathrm{H}_{2} \mathrm{O}\right)_{x}$, substituted bipy ligands, and NEM ( $N$-ethylmorpholine) in DMF. $^{184}$

The introduction of carboxylic groups on bipyridine ligands can be a valuable asset for dye-sensitized solar cell applications of ruthenium complexes. Thus, the ruthenium complexes $\left[\mathrm{RuCl}_{2}(\mathrm{dcmb})_{2}\right] 266\left(\mathrm{dcmb}=4,4^{\prime}\right.$-dimethoxycarbonyl-2,2' bipyridine, 265) and $\left[\mathbf{R u}(\mathbf{d c m b})_{3-n}(\mathbf{t b b p y})_{n}\right]\left(\mathbf{P F}_{6}\right)_{2}(n=0-$ 3) $\mathbf{2 7 0}$ could be synthesized with high efficiency by using a microwave-assisted synthetic procedure (Scheme 68). ${ }^{185}$ The positive effect of microwave irradiation was impressive as $\mathbf{2 6 6}$ could be isolated in $90 \%$ yield after $1 \mathrm{~h}$ heating under microwave irradiation, whereas the same complex was obtained in $78 \%$ yield after $50 \mathrm{~h}$ of conventional thermal heating. Interestingly, in 2006, Anderson et al. reported the microwaveassisted reaction of debpy (debpy $=4,4^{\prime}$-diethoxycarbonyl$2,2^{\prime}$-bipyridine) with $\mathrm{RuCl}_{3}$ which led to the formation of $\left[\mathbf{R u}(\text { bipy })_{3}\right]\left[\mathbf{P F}_{6}\right]_{2}$ through an unexpected decarboxylation of the ligand. ${ }^{186}$

Microwave irradiation of racemic cis-polypyridine and cispolyphenanthroline ruthenium complexes $\mathbf{2 7 1}$ in the presence of enantiopure methyl $p$-tolyl sulfoxide produced the corresponding ruthenium bis(diimine) sulfoxide complexes 272 or 273 with high diastereoselectivity $(73-76 \%$ de) and with a reduced reaction time compared to conventional heating (Scheme 69). ${ }^{187}$

Microwave irradiation of 2 equiv of tridentate $N, N, N$-dqp and dqp $\mathrm{NH}_{2}$ ligands (dqp = 2,6-di(quinolin-8-yl)pyridine 274, 
Scheme 69. Diastereoselective Formation of Polypyridine Ruthenium Complexes by Microwave Heating
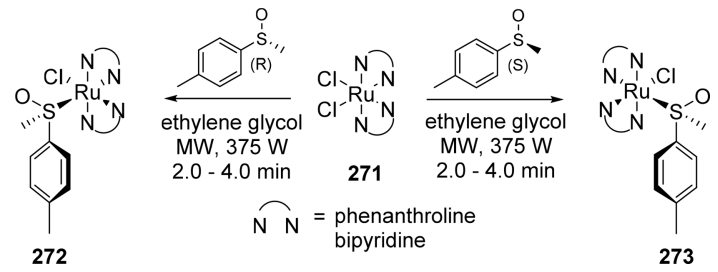

$\mathrm{dqpNH}_{2}=$ 2,6-di(quinolin-8-yl)-4-aminopyridine 275) with 1 equiv of $\left[\mathrm{Ru}(\mathrm{DMSO})_{4} \mathrm{Cl}_{2}\right] \mathbf{2 5 5}$ in ethylene glycol at $196{ }^{\circ} \mathrm{C}$ led to the formation of corresponding $\left[\mathrm{Ru}(\mathrm{dqp})_{2}\right]\left[\mathrm{PF}_{6}\right]_{2} \mathbf{2 7 8}$ and $\left[\mathbf{R u}\left(\mathbf{d q p N H}_{2}\right)_{2}\right]\left[\mathbf{P F}_{6}\right] \mathbf{2 7 9}$ in $87 \%$ and $22 \%$ yield, respectively (Scheme 70$){ }^{188}$ This procedure was unsuccessful

Scheme 70. Synthesis of Ruthenium Complexes with Tridentate Ligands

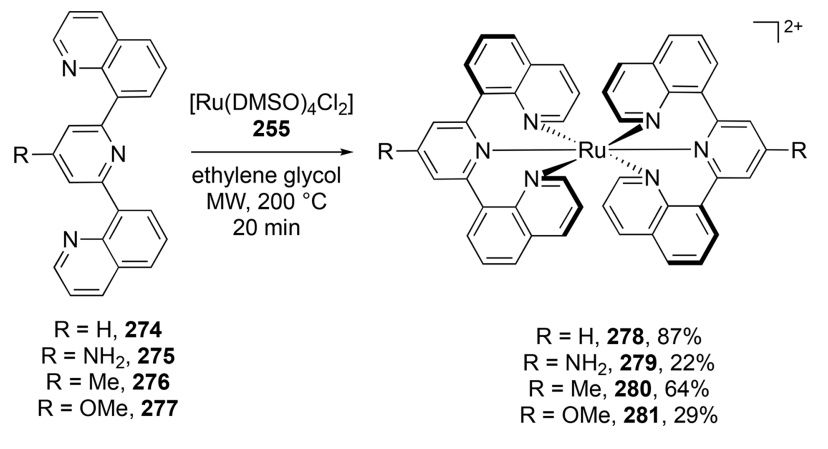

with dqp $\mathrm{CO}_{2} \mathrm{Et}$ (4-ethyl-carboxy-2,6-di(quinolin-8-yl)pyridine); only traces of product were detected. ${ }^{189}$ These complexes showed unprecedented ${ }^{3} \mathrm{MLCT}$ (metal to ligand charge transfer) lifetimes between 3 and $5.5 \mu \mathrm{s}$ in roomtemperature solution, which was a promising starting point for the construction of molecular arrays for applications in artificial photosynthesis. A similar synthesis using microwave irradiation was applied to form meridional ( $m e r-$ ) bistridentate ruthenium(II) complexes based on a dqp ligand containing inert functional groups. ${ }^{190}$ Heating dqpPhMe 276 or dqpOMe 277 with $\left[\mathrm{Ru}(\mathrm{DMSO})_{4} \mathrm{Cl}_{2}\right]$ in ethylene glycol at $200{ }^{\circ} \mathrm{C}$ using an Initiator single mode microwave cavity led to the formation of the mer-ruthenium complexes in 64 and $49 \%$ yield, respectively. Ligands with more reactive functional groups ( $\mathrm{R}$ $=-\mathrm{NH}_{2},-\mathrm{NO}_{2},-\mathrm{CO}_{2} \mathrm{Et}$, or $-\mathrm{Br}$ ) led to the corresponding complexes in lower yields or to the production of inseparable mixtures of complexes. Interestingly, after $5 \mathrm{~min}$ of irradiating dqp and $\left[\mathrm{Ru}(\mathrm{DMSO})_{4} \mathrm{Cl}_{2}\right]$ in an ethylene glycol solution at $180{ }^{\circ} \mathrm{C}$ instead of $200{ }^{\circ} \mathrm{C}$, a mixture of cis- and trans-fac$\left[\mathrm{Ru}(\mathrm{dqp})_{2}\right]^{2+} \mathbf{2 7 8}$ was obtained. Purification by column chromatography produced the cis isomer in $56 \%$ and the trans in $12 \%$ yield. Notably, prolonged microwave heating of both isomers resulted in the isolation of the mer-isomer in quantitative yields.

Complexes featuring polystyrene-functionalized terpyridines 284 with varying molecular weights have also been prepared using microwave irradiation (Scheme 71). ${ }^{191}$ Three $^{\text {PS }} \boldsymbol{x}^{-}[\mathbf{R u}]$ PEG $_{70}$ (PS = polystyrene; PEG = polyethylene glycol) block copolymers have been obtained with conversions of up to $73 \%$ using optimized parameters: $1 \mathrm{~h}$ at $90{ }^{\circ} \mathrm{C}$ in chloroform or in a $\mathrm{THF} / \mathrm{MeOH}$ mixture. While reduction of $\mathrm{Ru}(\mathrm{III})$ into $\mathrm{Ru}(\mathrm{II})$ requires harsh conditions under conventional heating, microwave irradiation enabled a decrease in reaction time and an increase in yield.

The use of microwave irradiation can also be valuable for the selective formation of organo-ruthenium species. During the conventional synthesis of $[\mathrm{Ru}(\mathrm{tctpy})(\mathrm{C}-\mathrm{N})(\mathrm{NCS})] 286$ (tctpy = tricarboxylterpyridine, $\mathrm{C}-\mathrm{N}=$ cyclometallating ligand such as 2-phenylpyridinato or 2-(4-(2-phenylethynyl)phenyl)pyridinato) starting from $[\mathrm{RuCl}(\mathrm{Me}-\mathrm{tctpy})(\mathrm{C}-\mathrm{N})] 285$, an inseparable stereoisomer of the desired complex was generated due to isomerization of the starting materials. ${ }^{192}$ Pure ruthenium complex 286 could be obtained selectively by inhibition of the undesired isomerization using microwave irradiation (Scheme 72).

Similarly, only one isomer of $\left[\mathbf{R u}(\mathbf{2 8 8})_{3}\right]\left[\mathbf{P F}_{6}\right]_{2}$ complexes 289 was obtained by the reaction of 4-alkoxycarbonylsubstituted unsymmetrical bipyridine ligands 288 with ruthenium(III) chloride in ethylene glycol under microwave irradiation (Scheme 73). ${ }^{193}$ This procedure was the first example of selective synthesis of the fac-isomer when using disconnected ligands. The authors could show that the position of the carboxyl group on the ligand played an important role for the stereochemistry of the complex since moving the ester from position $\mathrm{C}_{4}$ to $\mathrm{C}_{5}$ was detrimental to the fac/mer selectivity.

Microwave synthesis of polypyridyl ruthenium complexes was used to form a rare $\left[\mathbf{R u}_{2}(\mathbf{2 9 0})_{3}\right]^{4+}$ triple helicate 291 which was shown to interact with DNA. The complex could be separated efficiently into P- and M-enantiomers by DNA-based affinity chromatography. Initially, $\mathrm{RuCl}_{3}$ and the rigid quaterpyridine derivative $290\left(5,5^{\prime \prime}\right.$-dimethyl$2,2^{\prime}: 5^{\prime}, 3^{\prime \prime}: 6^{\prime \prime}, 2^{\prime \prime \prime}$-quaterpyridine) in a $2: 3$ ratio were heated in $\mathrm{EtOH}$ at reflux for 2 weeks, resulting in a complex mixture of products. When the reaction was performed under microwave irradiation (pressure vessel) at $225{ }^{\circ} \mathrm{C}$ in ethylene glycol for $4.5 \mathrm{~h}$, complex 291 was recovered in $36 \%$ yield (Scheme 74). ${ }^{194}$ Although $36 \%$ is a moderate yield, it is by far better than the $1 \%$ yield previously reported for the synthesis of a dinuclear ruthenium(II) helicate. ${ }^{195}$

Scheme 71. Synthesis of Heteroleptic Copolymer Complexes

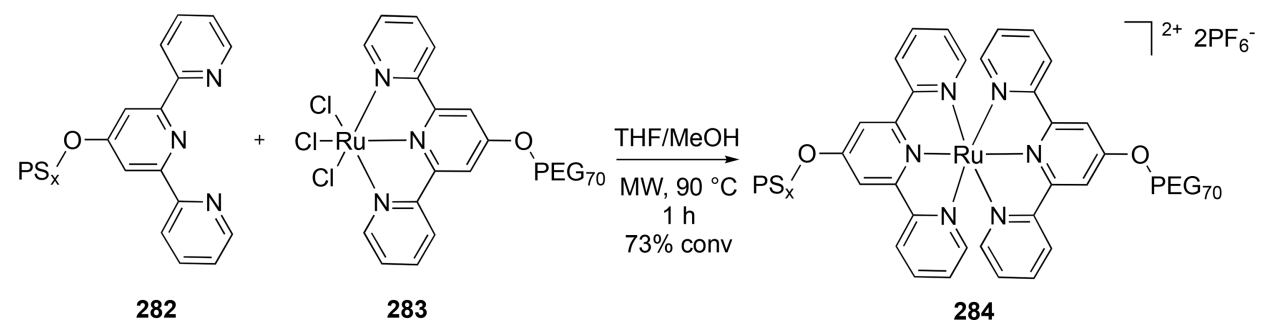


Scheme 72. Microwave Inhibition of Isomerization during the Synthesis of $[\mathrm{Ru}(\operatorname{tctpy})(\mathrm{C}-\mathrm{N})(\mathrm{NCS})]$ Complexes

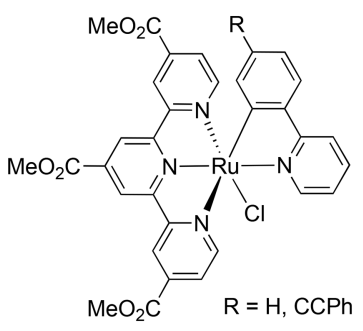

285

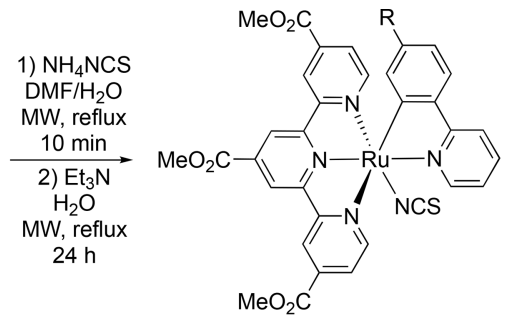

286

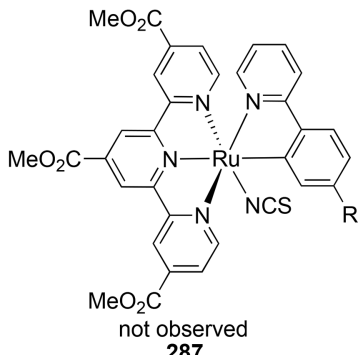

287
Scheme 73. Microwave Synthesis of fac-Isomer of Ruthenium Complexes

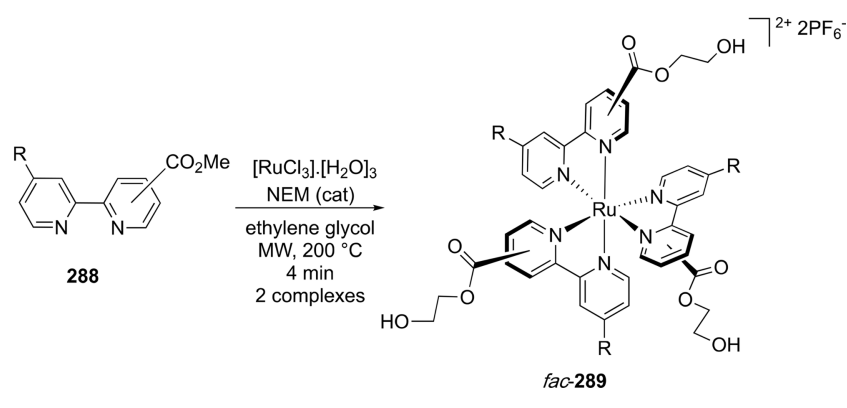

Scheme 74. Use of Microwave Irradiation for the Formation of the $\left[\mathrm{Ru}_{2} \mathrm{~L}_{3}\right]\left[\mathrm{PF}_{6}\right]_{4}$ Triple Helicate

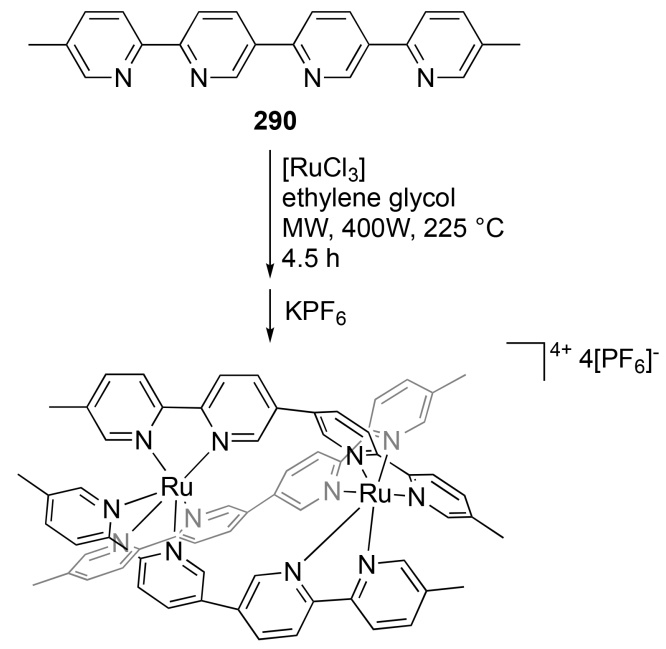

$291,36 \%$

In view of introducing a bridging group between two organometallic moieties, a Sonogashira cross-coupling reaction between bromoruthenium complex 292 and triisopropylsilylacetylene (TIPSA) was employed to yield the ethynylfunctionalized product $\left[\mathrm{Ru}(\text { tipsepd)(bipy) })_{2}\right]\left[\mathrm{PF}_{6}\right] 293$ (tipsepd $=(($ triisopropylsilyl $)$ ethynil $)$-2,4-pentanedionate ion $)$ (Scheme 75). ${ }^{196}$ When compared to classical conditions, microwave irradiation allowed for a reduction in the reaction time from 4-5 days to $1 \mathrm{~h}$, along with an increase in yield (from $40 \%$ to $62 \%$ ).

In the search for new ligands with similar or higher acidity than pyridine-based diamines, a series of homoleptic trischelated ruthenium(II)-arylazoimidazole complexes 295 were synthesized by microwave heating (Scheme 76). ${ }^{197}$ After (i) dissolution of $\left[\mathrm{RuCl}_{3}\right] \cdot\left[\mathrm{H}_{2} \mathbf{O}\right]_{3}$ in super dry $\mathrm{EtOH}$ and heating
Scheme 75. Sonogashira Coupling with Functionalized Bipyridine Complex under Microwave Heating

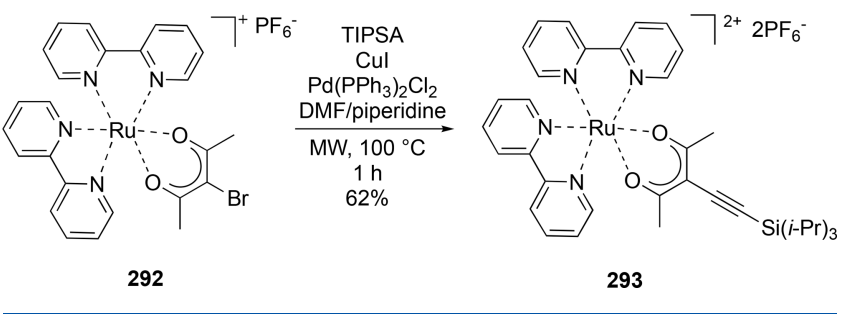

Scheme 76. Formation of Arylazoimidazoles Ruthenium Complexes under Microwave Heating

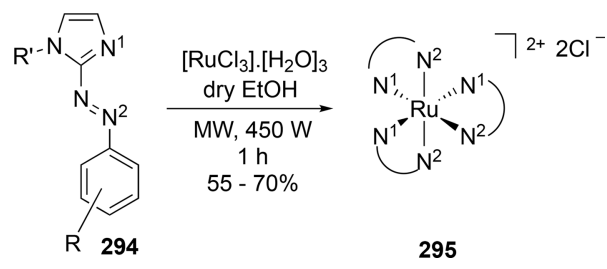

under MW irradiation for $3 \times 5 \mathrm{~min}$ and (ii) addition of the ligand 294 previously dissolved in $\mathrm{EtOH}$ and heating under MW irradiation for another $3 \times 5 \mathrm{~min}$, complexes 295 were recovered in moderate to good yields.

A highly efficient microwave-assisted synthesis of paddlewheel tetrakis(diaryltriazenido)diruthenium complexes 297, which possess interesting redox properties, was described in 2010 by the Jiménez-Aparicio group (Scheme 77). ${ }^{198}$ These

Scheme 77. First Microwave Synthesis of Multiple MetalMetal Bond Paddlewheel Compounds

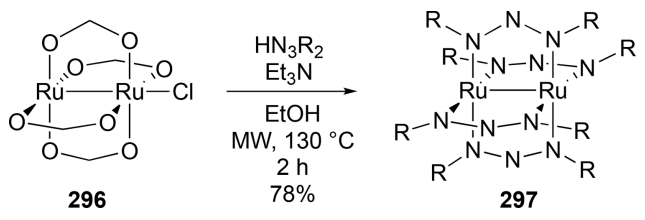

compounds were rarely described in the literature mainly because of the complexity of their synthesis, which requires five synthetic steps and up to 3 days to recover the complex in moderate yield. Using microwave irradiation enabled a reduction of the reaction time to $2 \mathrm{~h}$, with the yield increased to $78 \%$ ( $90 \%$ for a reaction time of $8 \mathrm{~h}$ ) as well as avoidance of toxic reagents and solvents. The same benefits were found for the microwave-assisted substitution of the acetate groups of $\left[\mathrm{Ru}_{2} \mathbf{C l}(\mathrm{OAc})_{4}\right]$ by three different types of $\mathrm{N}, \mathrm{N}$-donor ligands (1,3-diaryltriazenide, 1,3-diarylformamidinate, and guanidinate). Six corresponding complexes were isolated in high 
Scheme 78. Synthesis of Different Ruthenium Complexes Starting from $\left[\mathrm{RuCl}_{3}\right] \cdot\left(\mathrm{H}_{2} \mathrm{O}\right)_{n}$

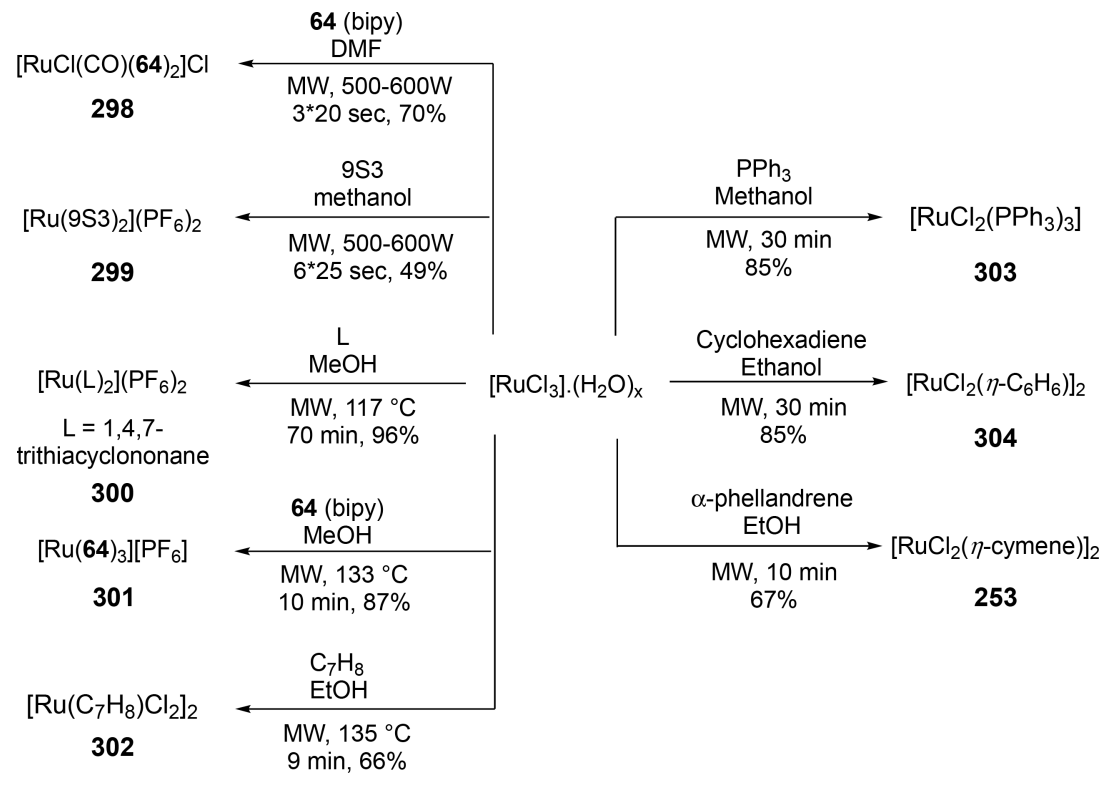

yields (90-98\%) with reaction times between $15 \mathrm{~min}$ and 8 h. ${ }^{199}$

As for rhenium complexes (see section 7.2), an early paper dealing with synthesis under microwave heating reported the synthesis of three different ruthenium complexes. ${ }^{125}$ By using the same equipment, the microwave irradiation of $\left[\mathrm{RuCl}_{3}\right]$. $\left(\mathrm{H}_{2} \mathrm{O}\right)_{n}$ and $\mathrm{PPh}_{3}$ in $\mathrm{MeOH}$ for $30 \mathrm{~min}$ provided $\left[\mathbf{R u C l}_{2}\left(\mathbf{P P h}_{3}\right)_{3}\right] \mathbf{3 0 3}$ in $85 \%$ yield (Scheme 78 ). Similarly, $\left[\operatorname{RuCl}_{2}\left(\eta-\mathrm{C}_{6} \mathbf{H}_{6}\right)\right]_{2} 304$ and $\left[\operatorname{RuCl}_{2}(\eta \text {-cymene })\right]_{2} 253$ were synthesized in 85 and $67 \%$ yield, respectively, by using EtOH as the solvent and cyclohexadiene or $\alpha$-phellandrene as ligand precursors that were oxidized during the process. In comparison with the literature, 3 to $4 \mathrm{~h}$ was necessary under conventional reflux conditions, while less than 1 min was required with microwave heating. Remarkably, $[\operatorname{RuCl}(\mathbf{C O})$ (bipy) $)_{2}$ Cl 298 was isolated in 1 week using conventional synthesis but was obtained in only $1 \mathrm{~min}(70 \%$ yield) when using microwave irradiation in an autoclave $(500-600 \mathrm{~W}) .^{91}$ $\left[\mathbf{R u}(\mathbf{9 S 3})_{2}\right]\left[\mathrm{PF}_{6}\right]_{2} 299$ (9S3 = 1,4,7-trithiacyclononane) was also synthesized in a few minutes in $49 \%$ yield, while it could not be obtained directly using conventional reflux conditions. Similarly, $\left[\mathbf{R u}(\text { bipy })_{3}\right]\left[\mathbf{P F}_{6}\right] 301$ and $\left[\mathrm{Ru}\left(\mathrm{C}_{7} \mathbf{H}_{8}\right) \mathrm{Cl}_{2}\right]_{2} 302$ $\left(\mathrm{C}_{7} \mathrm{H}_{8}=\right.$ cycloheptatriene $)$ were also synthesized in yields up to $87 \%$ and in short times (9 to $10 \mathrm{~min}$ ) via microwave activation. ${ }^{200}$ Notably, this was the first reported use of a pressure-controlled microwave oven for the synthesis of organometallic species.

In 2009, the Clarke group described the microwave synthesis of new chiral catalyst 306 for the enantioselective hydrogenation and transfer hydrogenation of bulky and poorly reactive ketones. $^{201}$ It was the first time that a ruthenium complex featuring a chiral tridentate $\mathrm{P}^{\wedge} \mathrm{N}^{\wedge} \mathrm{NH}_{2}$-type ligand was used in this enantioselective reaction. Ruthenium complex 306 was synthesized in $83 \%$ yield by heating the ligand and $\left[\mathrm{RuCl}_{2}(\mathrm{DMSO})_{4}\right]$ under microwave irradiation (Biotage Initiator Microwave) in THF for $15 \mathrm{~min}$ at $120{ }^{\circ} \mathrm{C}$ (Scheme 79).

In 2014, synthesis of $\left[\mathrm{Ru}_{2} \mathrm{Br}\left(\boldsymbol{\mu}-\mathrm{O}_{2} \mathrm{CC}_{6} \mathbf{H}_{4} \mathbf{M e}\right)_{4}\right] 308$ with the methyl group in either ortho, meta, or para position was described using three different methods: microwave irradiation

Scheme 79. Microwave Synthesis of a $\mathrm{P}^{\wedge} \mathrm{N}^{\wedge} \mathrm{NH}_{2}$ Ruthenium Complex
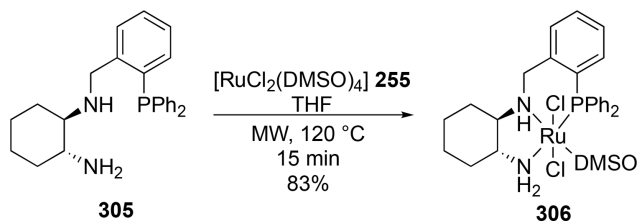

(using water as the solvent), solvothermal synthesis (using an autoclave and $\mathrm{MeOH}$ as the solvent), and conventional synthesis (at reflux in a $\mathrm{MeOH} / \mathrm{H}_{2} \mathrm{O}$ mixture). ${ }^{202}\left[\mathbf{R u}_{2} \mathbf{B r}\right.$ $\left.(\mathrm{OAc})_{4}\right] 307$ was used as the ruthenium source and $o-, m-$, or p-toluic acid as ligand precursors (Scheme 80). All of the

Scheme 80. Microwave Synthesis of New Bromidotetracarbocyclatodiruthenium(II,II) Complexes

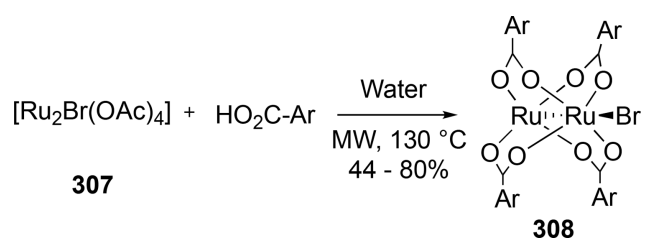

complexes adopted the typical paddlewheel structure (two ruthenium supported by four carboxylate ligands), and cationic $\left[\mathrm{Ru}_{2}\left(\mu-\mathrm{O}_{2} \mathrm{CC}_{6} \mathrm{H}_{4} \mathrm{Me}\right)_{4}\right]^{+}$units were bridged by bromide anions giving infinite zigzag chains. Interestingly, although the reaction yield with $m$-toluic acid was better via the microwave-assisted procedure, those using conventional heating were slightly better for $o$ - and $p$-toluic acid. The same study was carried out for the synthesis of $\left(\left[\mathrm{Ru}_{2} \mathrm{Cl}(9\right.\right.$ atc $\left.\left.\left.)_{4}\right] \cdot 2 \mathrm{H}_{2} \mathrm{O}\right]\right)_{n}(9$-atc $=9$-anthracenecarboxylic acid $){ }^{203}$ In this case, heating a solution of $\left[\mathrm{Ru}_{2} \mathbf{C l}(\mathbf{O A c})_{4}\right]$ and 9-atc in a mixture of $\mathrm{MeOH} /$ water at reflux for $4 \mathrm{~h}$ was more efficient than microwave irradiation as the expected complex product was recovered in $70 \%$ and $56 \%$, respectively.

Generally, synthesis of $\left[\mathrm{Ru}(p\right.$-cymene $\left.)(\text { azole })_{2} \mathrm{Cl}\right] \mathrm{Cl} 310$ 313 (azole $=1$-Me-imidazole, $1 \mathrm{H}$-pyrazole, $1 \mathrm{H}$-benzimidazole, 
Scheme 81. Microwave-Enhanced Synthesis of N-Heterocycle-Containing Ruthenium Species

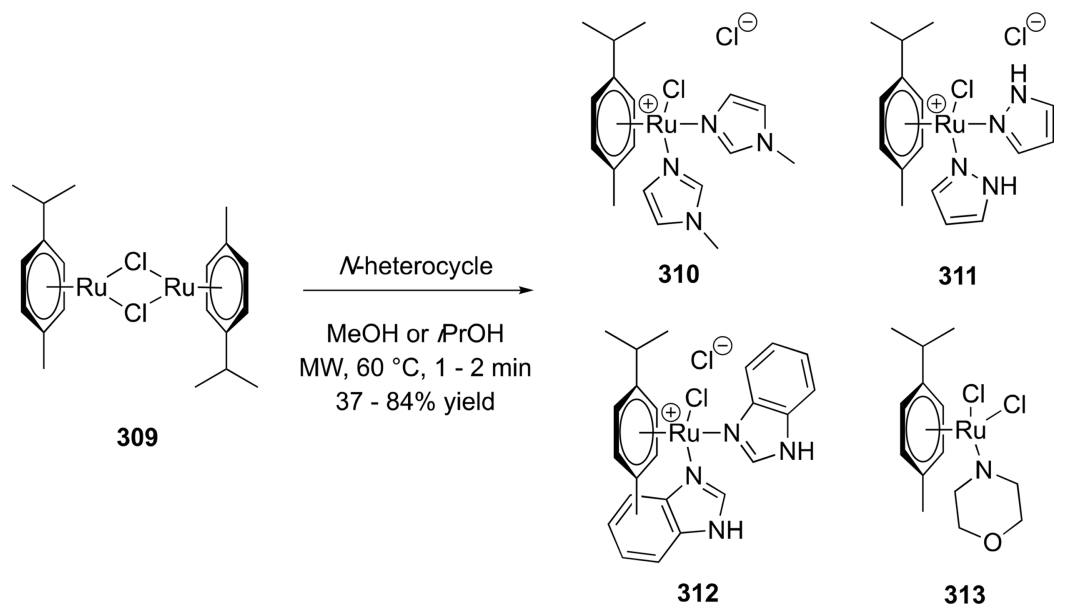

Scheme 82. Microwave Synthesis of $\left[\mathrm{Ru}\left(\mathrm{Cp}^{\wedge}\right)_{2} \mathrm{Cl}_{2}\right]_{2}$
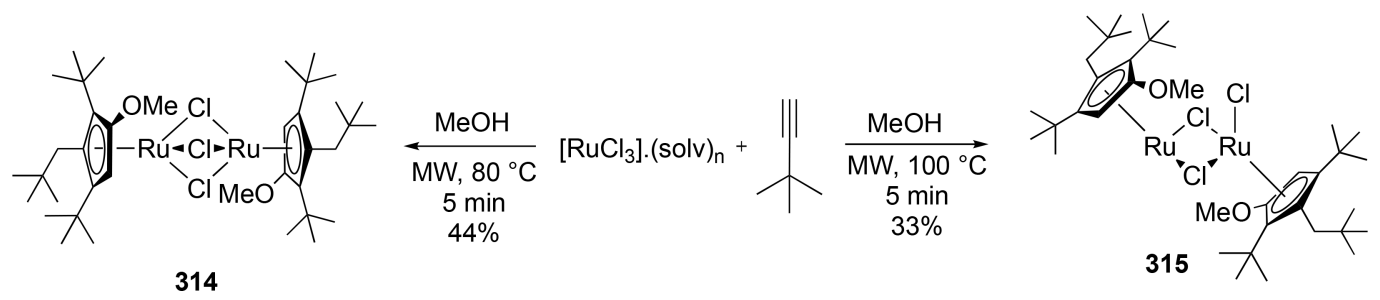

Scheme 83. Microwave Synthesis of a Tp Sandwich Compound

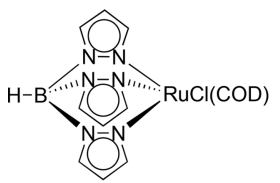

316

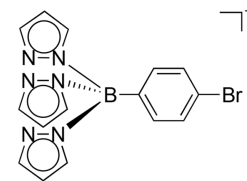

317

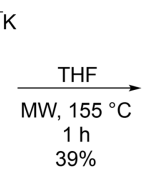

$39 \%$

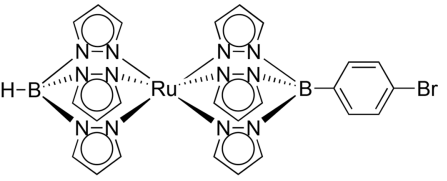

318 or morpholine) is performed by refluxing a solution of $\left[\mathrm{Ru}(\text { arene }) \mathrm{Cl}_{2}\right]_{2} 309$ for $7-18 \mathrm{~h}$ in the presence of the $\mathrm{N}$ containing heterocycle. The reaction time was, however, drastically decreased to only 1 or $2 \mathrm{~min}$ by heating the reaction mixture at $60{ }^{\circ} \mathrm{C}$ under microwave irradiation (Scheme 81) (CEM Explorer 12 Hybrid apparatus). ${ }^{204}$ The yields were higher than those obtained by a conventional synthesis $(37-84 \%$ vs $32-75 \%)$.

Dinuclear complexes $\left[\mathbf{R u C l}_{2}(p \text {-cymene })\right]_{2}$ and $\left[\mathbf{R u C l}_{2}(\text { cyclopentadienyl })\right]_{2}$ are important starting materials for the synthesis of other ruthenium complexes. Standard syntheses of these complexes were performed by heating the metal source and the ligand in an alcoholic solvent for several hours at reflux. In 1990, a microwave apparatus was used to develop a more efficient synthesis of $\left[\mathbf{R u C l}_{2}(p \text {-cymene })\right]_{2}$ (Scheme 78). ${ }^{125}$ In 2013, reaction conditions were further optimized to obtain the complex in only $30 \mathrm{~s}$ of microwave heating at $140{ }^{\circ} \mathrm{C}$ (by using a Biotage initiator 2.0). ${ }^{205}$ The complex was obtained in $86 \%$ yield, and only 2.5 equiv of the diene precursor was used instead of the large excess employed in the previously described methods. Similarly, $\left[\mathbf{R u C l}_{2}\left(\mathbf{C p}^{*}\right)\right]$ could be synthesized in $72 \%$ yield by microwave heating at 100 ${ }^{\circ} \mathrm{C}$ for 5 min while taking particular care to exclude air from the reaction mixture. Interestingly, $\left[\mathrm{RuCl}_{2}\left(\mathrm{Cp}^{\wedge}\right)\right]_{2} 315\left(\mathrm{Cp}^{\wedge}\right.$ = "Cp roof ligand", 2,4-di-tert-butyl-3-(2,2-dimethylpropyl)-1methoxycyclopentadienyl) could be formed in situ by a metal- mediated multicomponent reaction between tert-butylacetylene and $\mathrm{MeOH}$ (Scheme 82). Heating the reaction mixture at $100{ }^{\circ} \mathrm{C}$ for $5 \mathrm{~min}$ led to the formation of the desired complex, whereas decreasing the temperature to $80{ }^{\circ} \mathrm{C}$ induced the formation of the unexpected ruthenium complex 314, the formation of which could not be rationalized.

Hydrido-tris(pyrazolyl)borate ( $\mathrm{Tp}$ ) is an analogue of the widely used $\mathrm{Cp}$ ligand. The Metzler-Nolte group used it for the production of ruthenium complexes in 2011. Starting from a solution of $[\mathrm{RuTp}(\mathbf{C O D}) \mathbf{C l}] 316$ and $p-\mathrm{BrC}_{6} \mathrm{H}_{4} \mathrm{TpK} 317$ in THF, $\left[\mathbf{R u}\left(p-\mathbf{C}_{6} \mathbf{H}_{4} \mathbf{T} \mathbf{p}\right) \mathbf{T p}\right] \mathbf{3 1 8}$ was synthesized by heating the reaction mixture for $1 \mathrm{~h}$ at $155^{\circ} \mathrm{C}$ under microwave irradiation (Scheme 83). ${ }^{206}$ In classical conditions, the reaction mixture was heated for $12 \mathrm{~h}$ to obtain the complex in lower yield (19\%), probably because of starting material decomposition. Interestingly, 318 was then converted to $\left[\operatorname{RuTp}\left(p-\left(\mathrm{CO}_{2} \mathrm{H}\right)\right.\right.$ $\left.\mathrm{C}_{6} \mathrm{H}_{4} \mathrm{Tp}\right)$ ], which was coupled to the pentapeptide Enkephalin using solid-phase synthesis to provide $[\operatorname{RuTp}(p-(\mathrm{CO}-\mathrm{Tyr}-\mathrm{Gly}-$ Gly-Phe-Leu-OH)- $\left.\left.\mathrm{C}_{6} \mathrm{H}_{4} \mathrm{Tp}\right)\right]$ as the first example of a ruthenium $\mathrm{Tp}$ sandwich bioconjugate.

Fast ligand modification around the ruthenium center of $\left[\mathrm{RuCl}(\mathrm{Cp})\left(\mathrm{PPh}_{3}\right)_{2}\right] 319$ could be performed efficiently using microwave irradiation. Heating a mixture of dppm 62 and various thiolate ligands with 319 in diethylene glycol produced the corresponding $[\mathbf{R u}(\mathbf{C p})(\mathbf{d p p m}) \mathrm{SR}]$ complexes 320 in good yields (Scheme 84 ). ${ }^{207}$ Complexes were recovered after 
Scheme 84. Microwave Synthesis of Cyclopentadienyl Bisphosphine Ruthenium(II) Thiolate Complexes

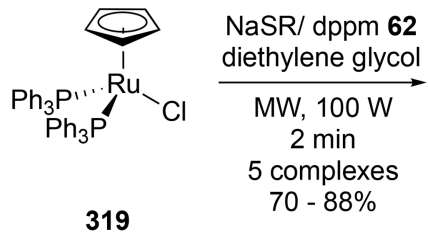

319

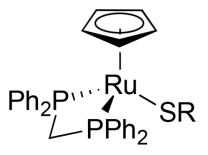

$\mathrm{R}=\mathrm{Ar}, \mathrm{Bn}$, ester, amino acid 320 only 2 min of heating with a Labwell Microwell 10 reactor operated at $100 \mathrm{~W}$, in $10 \mathrm{~min}$ while using a household microwave oven and in $2 \mathrm{~h}$ when using conventional heating conditions. Similar yields were obtained independently of the chosen method. Direct insertion of thiophenol into the coordination sphere of 319 proved difficult since numerous side products were formed. Such examples proved the need for a stabilizing bidentate dppm ligand on the metal. Interestingly, the use of an amino acid thiolate ligand resulted in the selective formation of a $\mathrm{Ru}-\mathrm{S}$ bond under microwave activation, while a mixture with a COO linkage was observed in classical synthesis.

Contrary to what was observed with $\mathrm{Cp}$ ligands, microwave heating (Biotage initiator) of ruthenium complexes featuring arene ligands in the presence of $\mathrm{L}-\mathrm{L}^{\prime}$ bidentate ligands ( $\mathrm{P}-$ $\mathrm{P}^{\prime}-, \mathrm{N}-\mathrm{N}^{\prime}-, \mathrm{P}-\mathrm{N}-$, and $\mathrm{P}-\mathrm{S}-$ chelate ligands) led to the displacement of the arene, resulting in complexes of the general formula $\left[(\right.$ arene $\left.) \mathbf{R u}(\mu-\mathbf{C l})_{3} \operatorname{RuCl}\left(L-L^{\prime}\right)\right] \quad 323$ (Scheme 85). ${ }^{208}$ Microwave irradiation of $\left[\mathbf{R u C l}_{2}(\text { arene })\right]_{2}$

Scheme 85. Microwave Synthesis of $[($ arene $) \operatorname{Ru}(\mu$ $\left.\mathrm{Cl})_{3} \mathrm{RuCl}(\mathrm{L}-\mathrm{L})\right]$ Complexes

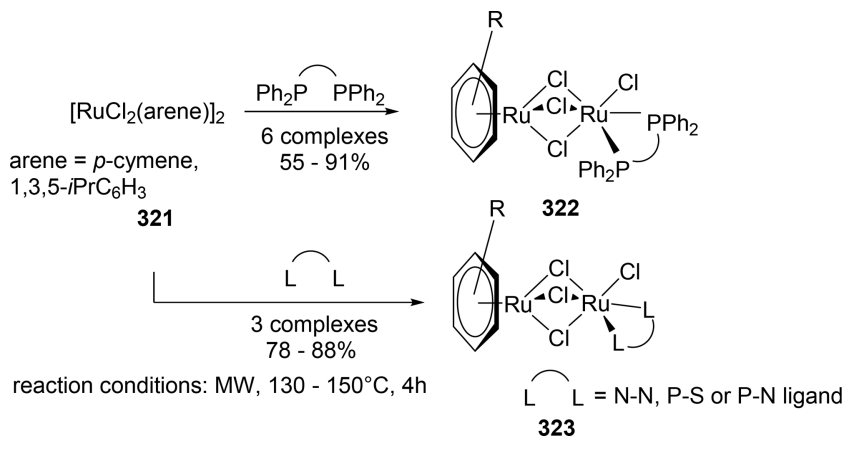

with diphosphine ligands in a THF solution at $130-150{ }^{\circ} \mathrm{C}$ for $4 \mathrm{~h}$ provided six dinuclear ruthenium complexes 322 in yields of $55-91 \%$, including $\left[\mathrm{Ru}(\mu-\mathrm{Cl})_{3} \operatorname{RuCl}(\mu-(S, S)\right.$-DIOP $\left.)\right]$ $(\mathrm{DIOP}=(4 S, 5 S)-2,2-($ dimethyl-1,3-dioxolane-4,5-diyl $)$ bis(methylene)bis(diphenylphosphine)). Under classical reflux conditions in THF, $\left[\operatorname{RuCl}_{2}(p \text {-cymene })_{2}(\boldsymbol{\mu}\right.$-(S,S)-DIOP $\left.)\right]$ was formed, but a change of solvent to 2-ethoxyethanol allowed the isolation of the desired dinuclear complex $\left[\mathbf{R u}(\mu-\mathbf{C l})_{3} \mathbf{R u C l}(\boldsymbol{\mu}\right.$ $(S, S)$-DIOP $)]$, albeit in lower yield and longer reaction time than under microwave irradiation. The use of $\mathrm{N}-\mathrm{N}^{\prime}, \mathrm{P}-\mathrm{N}$, and $\mathrm{P}-\mathrm{S}-$ chelates was also efficient, providing corresponding complexes 323 in $78-88 \%$ yields.

A microwave-assisted synthesis of Shvo-type complexes 325 was developed in 2014 by the Mazzoni group. ${ }^{209}$ Hydroxycyclopentadienylruthenium hydride dimer complexes, known as Shvo-type complexes, are precursors of useful catalysts. When solutions of $\left[\mathrm{Ru}_{3}(\mathbf{C O})_{12}\right]$ and functionalized tetraarylcyclopentadienones 324 were heated in $\mathrm{MeOH}$ at $110^{\circ} \mathrm{C}$ in a "high-throughput rotor" microwave oven, corresponding complexes 325 were produced in $23-75 \%$ yields (Scheme 86 ). The most striking advantage of this approach is the reduction of the reaction time, from $40 \mathrm{~h}$ under classical solution conditions to $50 \mathrm{~min}$ via microwave irradiation.

There are few examples of microwave synthesis of osmium complexes described in the literature. In 2006, the microwaveassisted synthesis of $\left[\mathrm{Os}_{2} \mathrm{Cl}_{3}\left(\mathbf{P E t}_{2} \mathbf{P h}\right)_{6}\right] \mathbf{C l} 328$ was reported (Scheme 87). ${ }^{210}$ This complex was used as a synthon for the synthesis of various osmium complexes and was crucial for the synthesis of dihydrogen osmium complexes. Microwave heating enabled the isolation of $\mathbf{3 2 8}$ in only $5 \mathrm{~min}$ under aerobic conditions, while previous methods required refluxing in aqueous EtOH for 1 week. Interestingly, this publication was the first to describe the structure of the complex thanks to single-crystal X-ray diffraction.

The classical synthesis of arene(osmium) halide complexes is well-documented, and all the reported procedures require long reaction times (up to $100 \mathrm{~h}$ ) as well as high temperatures to achieve completion. ${ }^{211}$ Microwave heating at $110{ }^{\circ} \mathrm{C}$ of $\mathrm{Na}_{2} \mathrm{OsCl}_{6}$ with 1,3-cyclohexadiene in $\mathrm{EtOH}$ allowed the formation of $\left[\operatorname{OsCl}_{2}\left(\boldsymbol{\eta}-\mathbf{C}_{6} \mathbf{H}_{6}\right)\right]_{2} 329$ in high yield (97\%) with a shorter reaction time (Scheme 88).

Similarly to ruthenium complexes (see Scheme 81), [Os $(p$ cymene $\left.) \mathbf{C l}(\text { azole })_{2}\right] \mathbf{C l}$ (azole $=1$-Me-imidazole, $1 \mathrm{H}$-pyrazole, or $1 \mathrm{H}$-benzimidazole) could be synthesized by heating a polar alcohol solution of $\left[\mathrm{Os}(p\right.$-cymene $\left.) \mathrm{Cl}_{2}\right]$ with an $\mathrm{N}$-containing heterocyclic ligand at $80{ }^{\circ} \mathrm{C}$ with microwave activation. ${ }^{204}$ Only 1 or $2 \mathrm{~min}$ of reaction was necessary for efficient formation of the osmium complexes (54-78\% yield). Using morpholine (morph) in the reaction mixture provided the $\left[\mathrm{Os}(\boldsymbol{p}\right.$-cymene $)($ morph $\left.) \mathrm{Cl}_{2}\right]$ complex.

\subsection{Ultrasonic Irradiation for Iron Complexes}

Two different 2-ferrocenylpyrazoles 330 were methylated using ultrasound chemistry (Scheme 89). ${ }^{167}$ A solution of ferrocenyl complex 330, sodium hydride, and methyl $p$ -

Scheme 86. Synthesis of Shvo-Type Ruthenium Complexes

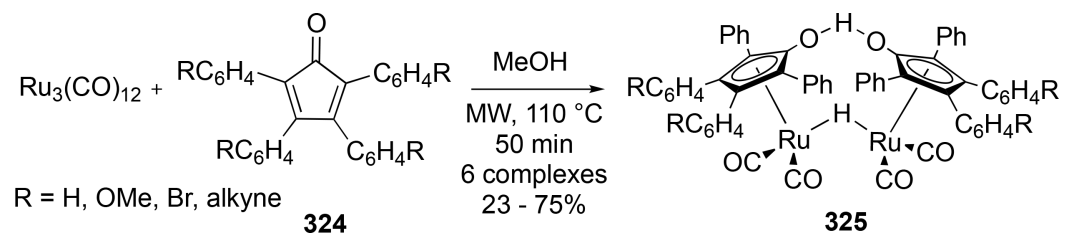


Scheme 87. Microwave Synthesis of a Useful Osmium Synthon

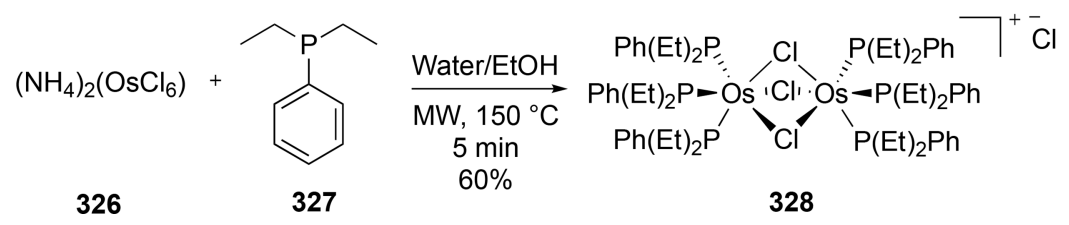

Scheme 88. Synthesis of $\left[\mathrm{OsCl}_{2}\left(\eta-\mathrm{C}_{6} \mathrm{H}_{6}\right)\right]_{2}$ Using Microwave Irradiation

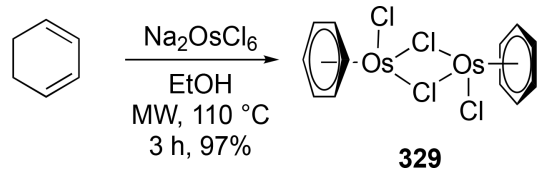

Scheme 89. Methylation of 2-Ferrocenylpyrazole under Ultrasonic Irradiation

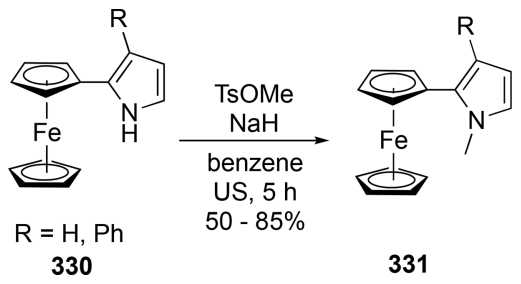

toluenesulfonate in benzene was sonicated for $5 \mathrm{~h}$ to produce the corresponding complexes 331 in 50 and $85 \%$ yield.

The Raghunathan group developed an expedient one-pot, four-component synthesis of ferrocene-grafted spiropyrrolidine heterocycles 336 that was assisted by ultrasonic treatment. ${ }^{212}$ A solution of the ferrocene-derived dipolarophile 335, ninhydrin 332, 1,2-phenylenediamine 333, and sarcosine 334 in $\mathrm{MeOH}$ was sonicated under refluxing conditions for 1 to $2 \mathrm{~h}$ to afford the desired complexes with high yield and high regioselectivity, as confirmed by X-ray diffraction (Scheme 90). The author suggested that the reaction proceeded via reaction of ninhydrin, 1,2-phenylenediamine, and sarcosine to form the azomethine ylide intermediate 338 , which then reacted with ferrocene derivative 335 via a $[3+2]$-cycloaddition. By reacting with 1-phenyl-3-ferrocenyl-3-prop-2-en-1-one derivatives, five similar complexes were formed in high yields. Under classical thermal conditions, 5 to $7 \mathrm{~h}$ was necessary to afford the desired ferrocene complexes 336 in lower yields.
The Penoni and Cravotto groups developed an ultrasoundassisted, one-pot, three-component reaction with ferrocenecarboxaldehyde 236 (Scheme 91). ${ }^{213}$ Allylindation and

Scheme 91. Ultrasound Allylation of

Ferrocenecarboxaldehyde

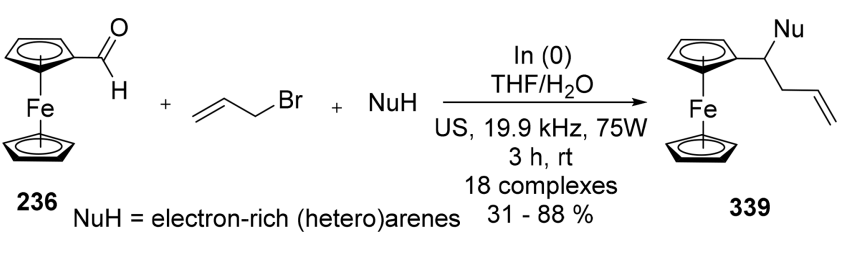

dehydrative alkylation of stabilized $\mathrm{C}$ - or $\mathrm{N}$-nucleophiles gave the corresponding functionalized ferrocenes 339 in good yields. Various ultrasonic baths were tested for the reaction, and the cup horns working at $19.9 \mathrm{kHz}(75 \mathrm{~W})$ were found to be the most efficient. When reacting with highly activated nucleophiles such as pyrrole and furan, the bisalkylated compound was the single product.

8.4. Syntheses of Iron and Ruthenium Complexes Using Photochemistry

In 2008, a short review written by Aranzaes and Astruc described the synthesis of various piano-stool organo-iron complexes $\left[\mathrm{FeCpL}^{1} \mathrm{~L}^{2} \mathrm{~L}^{3}\right]^{+}$341-343 $(\mathrm{L}=$ neutral two-electron ligand) by visible photolysis of $[\mathrm{FeCp}($ arene $)]\left[\mathrm{PF}_{6}\right]$ sandwich complexes 340 (Scheme 92). ${ }^{214}$ Arene displacement and nucleophilic substitution were performed under light irradiation and yielded the phosphino or acetonitrile complexes. When the nucleophilic substitution was attempted on $\left[\mathrm{FeCp}\left(\mathrm{C}_{6} \mathbf{M e}_{6}\right)\right][\mathrm{OH}]$ in water, the reaction led to the decomposition of the ferrocenyl complex. If all the reactions were first described with a $\mathrm{Cp}$ ring on the iron complex, the reaction conditions could also be used to form $\mathrm{Cp}^{*}$-containing iron derivatives.

\section{Scheme 90. Ultrasound Synthesis of Novel Ferrocene-Grafted Pyrrolidine Heterocycles}

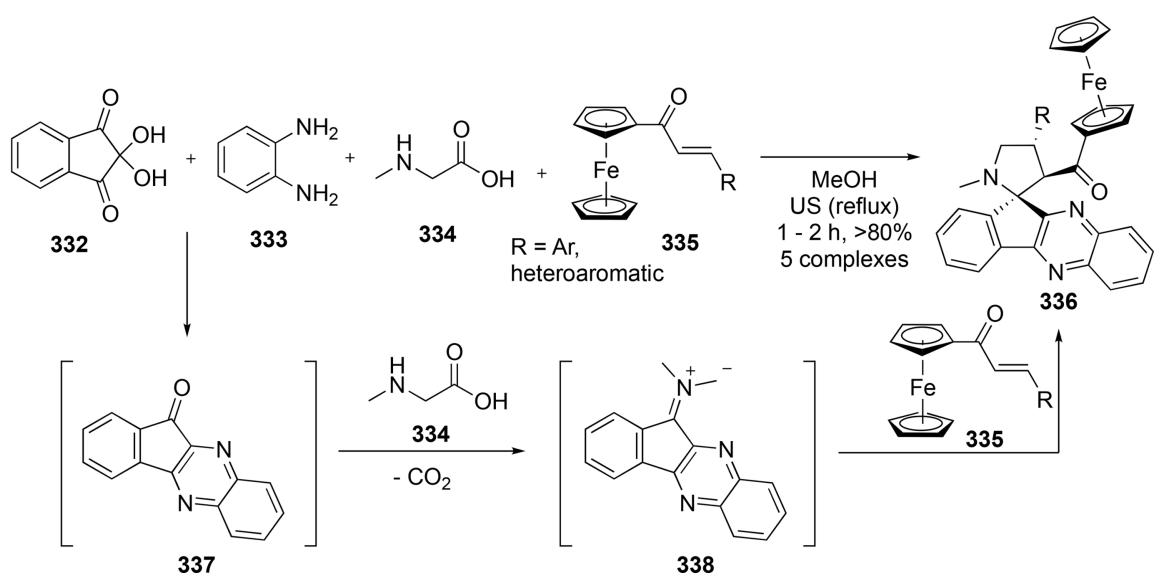


Scheme 92. Photochemical Access to Piano-Stool FeCp Analogue Complexes

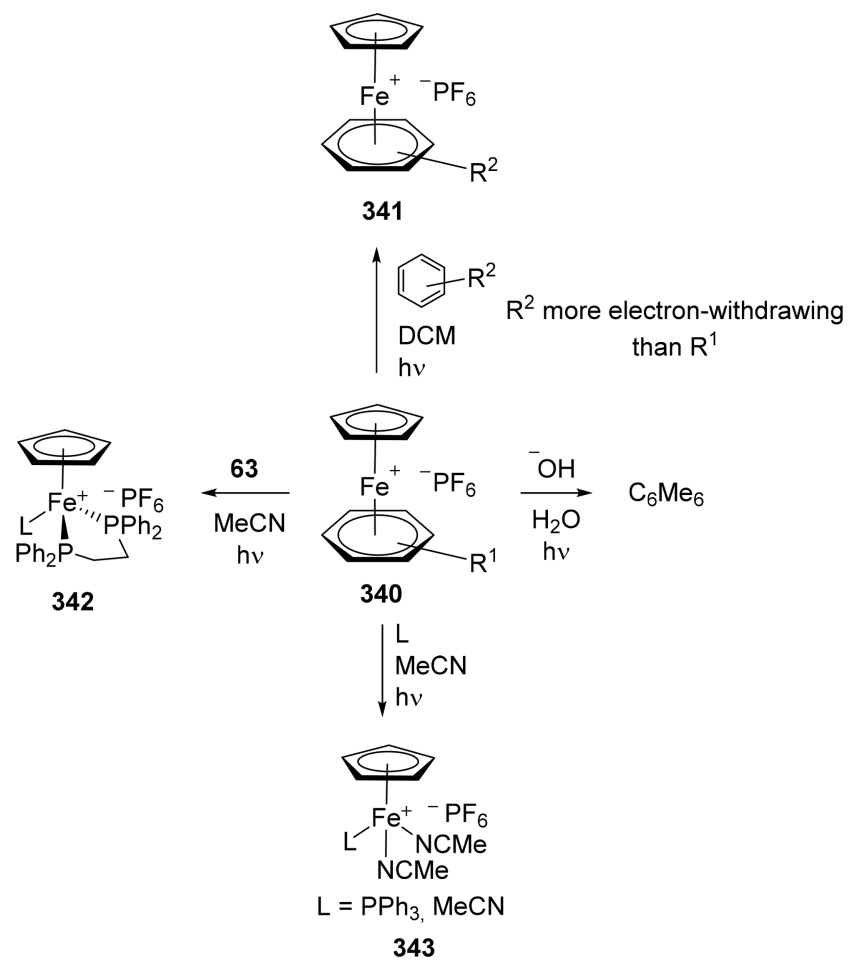

Interestingly, photolysis of $[\mathrm{FeCp}($ arene $)] \mathbf{P F}_{6}$ could be used to functionalize $\mathrm{Cp}$-iron complexes. In this context, the Astruc group described the photolysis of $\left[\mathrm{FeCp}\left(\eta^{6}\right.\right.$-toluene $\left.)\right]$ $\left[\mathrm{PF}_{6}\right] \mathbf{3 4 4}$ in 2009 as a general route to iron-alkynyl complexes 345 using terminal alkynes, through a decoordination of the toluene ligand (Scheme 93). ${ }^{215}$ The reaction was then extended to ferrocenylacetylene to yield bimetallic complexes 346-347 starting from 1,4-bis(ethynyl)benzene or ethynylferrocene.

Starting from ferrocenyl imines 348 and 349, ferrocenesubstituted 2-azetidinones 351 and 352 could be obtained predominantly as cis isomers in good yields after irradiation in the presence of alkoxychromium $(0)$-carbene complexes 350 (Scheme 94, reaction A). ${ }^{216}$ Contrastingly, irradiation of the ferrocenechromium $(0)$-carbene complex 353 with an imine under different reaction conditions was systematically unsuccessful, thus showing that this complex was photochemically inert (Scheme 94, reaction B). Alternative approaches to place a ferrocene moiety at the $\mathrm{C} 3$ position of the 2azetidinone ring failed. However, irradiation under $\mathrm{CO}$ pressure of aminochromium(0)-carbene complex 354 possessing a ferrocene group attached to its amino moiety gave azetidine complex 356 in low yield (23\%) (Scheme 94, reaction $\mathrm{C}$ ).

There are few reports of ruthenium complex synthesis via photochemistry. In 1989, Suen et al. subjected a series of ruthenium terpyridine complexes to photolysis and studied their ligand substitution. Photolysis (using a $275 \mathrm{~W}$ sunlamp) of $\left[\mathrm{Ru}(\text { terpy }) \mathrm{L}_{3}\right]^{2+} 357$ or $\left[\mathrm{Ru}(\text { terpy }) \mathrm{L}_{2} \mathrm{Cl}\right]^{+} 358(\mathrm{~L}=\mathrm{ACN}$, $\left.\mathrm{PPh}_{3}, \mathrm{Py}\right)$ in acetone or dichloromethane resulted in the efficient loss of the ligand and coordination of the solvent molecule (acetonitrile or propionitrile). When these nitriles were replaced by tetraethylammonium chloride, a chloride ligand was added to the metal center (359-360, Scheme 95). ${ }^{217}$ Column chromatography using alumina was used to purify the complexes, which were recovered in yields higher than $80 \%$. Surprising specificity of ligand loss was observed, in favor of the ligand in the axial position. This observation was explained by a photosubstitution theory based on the angular overlap model with the basic assumption defined by IUPAC as "the strength of a bond formed using atomic orbitals on two atoms is related to the magnitude of overlap of the two orbitals".

In 2009, Matsubara et al. described the irradiation of a solution of $\left[\mathrm{Ru}(\text { terpy)(bipy)(DMF) }]^{2+} 363\right.$ and triethylamine in THF using $436 \mathrm{~nm}$ monochromatic light for $2 \mathrm{~h}$ to generate the hydrido complex $[\mathbf{R u}(\text { terpy })(\text { bipy }) \mathbf{H}]^{+} 364$, which could be produced in quantitative yield (Scheme 96). ${ }^{218}$ After irradiation, the 1-benzyl-3-trifluoromethylpyridinium cation 365 was added to obtain the corresponding hydride reduction product 366. Investigations of the properties of $[\mathrm{Ru}($ terpy)(bipy)(DMF) $]^{2+} 363$ and $\left[\mathrm{Ru}\left(\text { terpy)(bipy) }\left(\mathrm{NEt}_{3}\right)\right]^{2+}\right.$ indicated that the formation of the hydrido ruthenium complex 364 proceeded via the excitation of the triethylaminoruthenium complex. In the absence of $\mathrm{NEt}_{3}$, irradiation of a solution containing complex 363 in THF resulted in a ligand exchange reaction to give the $\left[\mathbf{R u}(\text { terpy)(bipy)(THF) }]^{2+}\right.$ complex. Due to the low reformation of 363 and the large quantity of THF as compared to DMF, $[\mathbf{R u}($ terpy)(bipy)(THF) $]^{2+}$ was obtained in nearly quantitative yield.

Padhi and Tanaka demonstrated the photoredox behavior of complex $\left[\mathbf{R u}(\right.$ phbn $)\left(\right.$ bipy) $\left.{ }_{2}\right] \mathbf{P F}_{6} 368$ (phbn = 3-phenylbenzo$[\mathrm{b}][1,6]$ naphthyridine $).{ }^{219}$ Irradiation of the complex in the presence of 1 equiv of hydroquinone in a mixture of acetonitrile $/ \mathrm{Et}_{3} \mathrm{~N}$ produced the reduced $\left[\mathbf{R u}\left(\mathbf{p h b n H _ { 2 }}\right)\right.$ (bipy) $\left.)_{2}\right] \mathbf{P F}_{6} 369$ quickly and in good yield (Scheme 97, pathway A). Similarly, under irradiation, the reduction could be performed in acetonitrile with 1 equiv of $p$-chloranil, yielding 369 in $78 \%$ yield (Scheme 97, pathway B).

Scheme 93. Synthesis of Iron-Acetylide Complexes by Visible-Light Photolysis

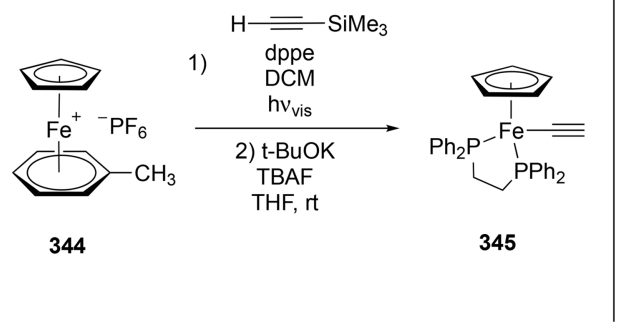


Scheme 94. Photochemical Synthesis of Ferrocene-Substituted 2-Azetidinone Complexes

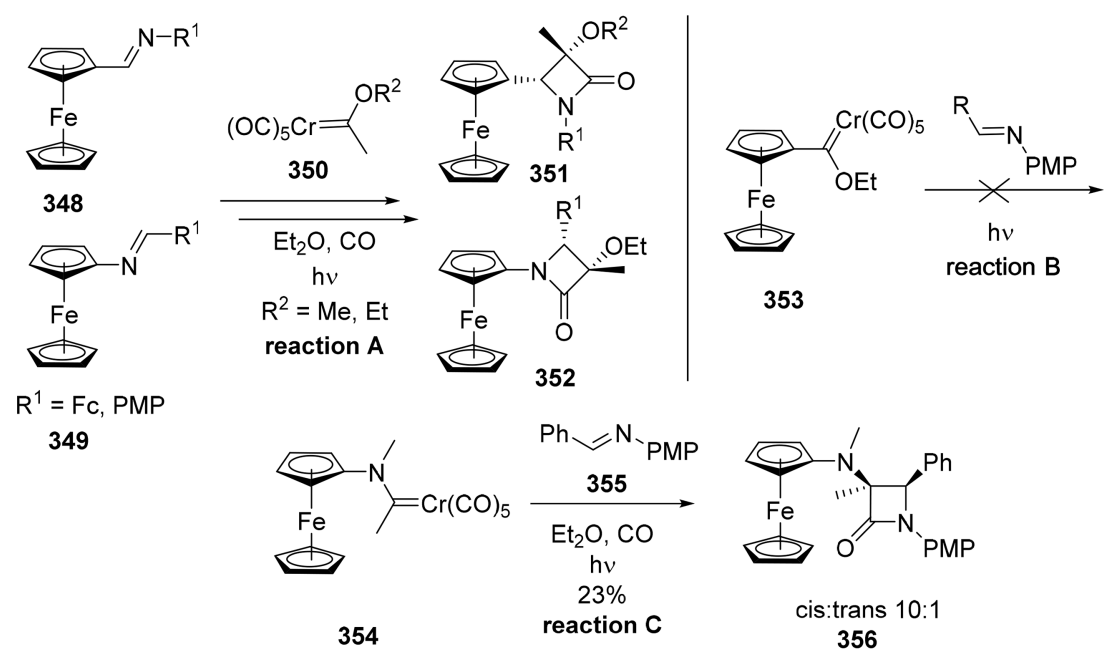

Scheme 95. Photochemical Ligand Substitution of $\left[\mathrm{Ru}(\text { terpy }) \mathrm{L}_{3}\right]^{2+}$ and $\left[\mathrm{Ru}(\operatorname{terpy}) \mathrm{L}_{2} \mathrm{Cl}\right]^{+}$

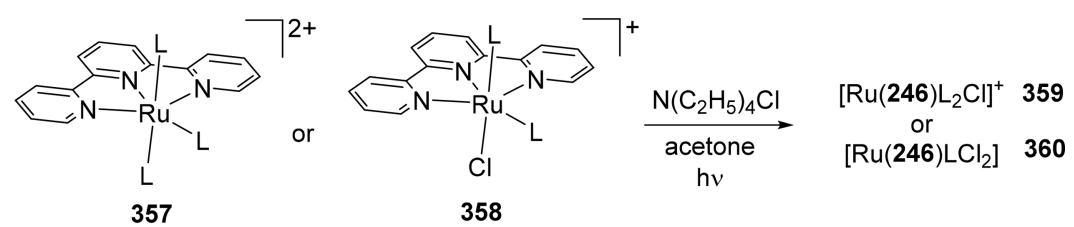

$\mathrm{L}=$ pyridine, $\mathrm{ACN}, \mathrm{PPh}_{3}$

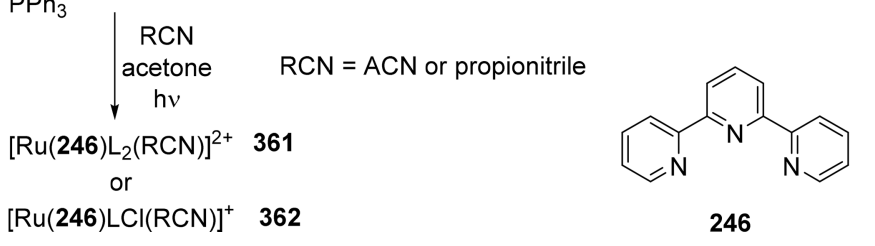

Scheme 96. Photochemical Formation of a Hydrido Ruthenium Complex by Irradiation of $[\mathrm{Ru}(\text { terpy })(\text { bipy })(\mathrm{DMF})]^{2+}$

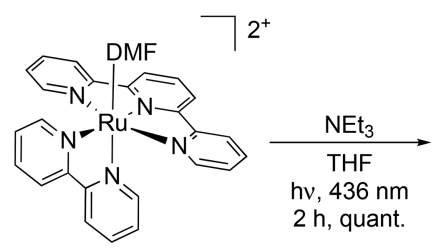

363

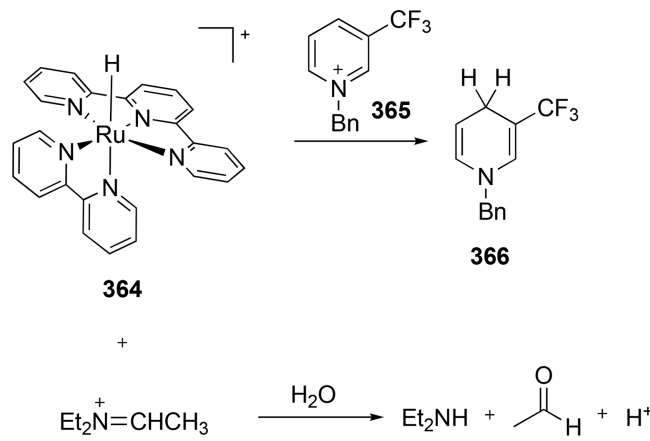

367
In 2007, Cooke proposed the photochemical preparation of various $\left[\mathbf{R u}(\mathrm{CO})_{4}\left(\boldsymbol{\eta}^{2}\right.\right.$-alkene) $]$ complexes (370) from $\left[\mathbf{R u}_{\mathbf{3}}(\mathbf{C O})_{\mathbf{1 2}}\right]$ (Scheme 98). ${ }^{220}$ The aim of this study was to demonstrate that under light excitation a metal-metal bond can be cleaved instead of the more classical metal-carbonyl bond breakage. The reaction could be performed under different conditions ranging from filtered high-intensity light to sunlight or conventional fluorescent lighting. Only a few minutes was necessary using a high-intensity light, while irradiation for days was required under fluorescent lighting of lower intensity. The methods were suitable for preparative- scale reactions (around $0.2 \mathrm{~g}$ ), and products were recovered in quantitative yield.

As for iron, photolysis of $[\mathrm{RuCp}(\text { benzene })]^{+} 371$ in acetonitrile at $313 \mathrm{~nm}$ led to the decoordination of the benzene and the quantitative formation of $\left[\operatorname{RuCp}(\mathrm{ACN})_{3}\right]^{+}$ 372, which could be isolated as a $\mathrm{PF}_{6}{ }^{-}$salt with a quantum yield of $0.4 .^{221}$ No photoreaction was observed when irradiating a solution of 371 in $\mathrm{CH}_{2} \mathrm{Cl}_{2}$ in the absence of ligands. The addition of phosphites $\left(\mathrm{P}\left(\mathrm{OCH}_{3}\right)_{3}\right.$ or $\left.\mathrm{P}(\mathrm{OEt})_{3}\right)$, however, yielded the corresponding $\left[\operatorname{RuCp}\left(\mathbf{P}(\mathbf{O R})_{3}\right)_{3}\right]^{+} 373$ complexes after photolysis (Scheme 99). Surprisingly, in the presence of $\mathrm{P}(\mathrm{OPh})_{3}$, only the starting materials were 
Scheme 97. Photoredox Reaction of the Ru Complex

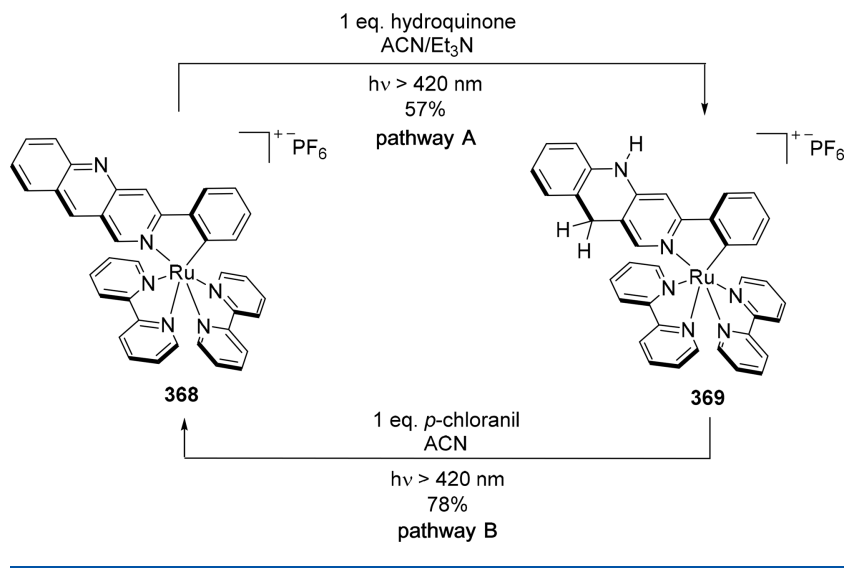

Scheme 98. Photochemical Transformation of $\left[\mathrm{Ru}_{3}(\mathrm{CO})_{10}\right]$

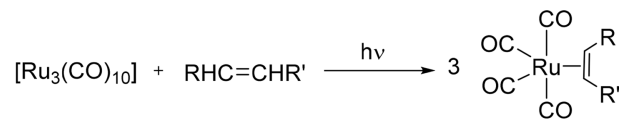

$$
\begin{aligned}
& \mathrm{RHC}=\mathrm{CHR}^{\prime} \text { : ethylene, methyl acrylate } \\
& \text { or dimethyl fumarate } \\
& 370
\end{aligned}
$$

recovered. This contrasted with results obtained for the photolysis of $[\mathrm{FeCp}(p$-xylene $)]$, which led to the efficient formation of $\left[\mathrm{FeCp}\left(\mathbf{P}(\mathbf{O P h})_{3}\right)_{3}\right]^{+}$. Notably, under thermal conditions, the trisacetonitrile complex 372 could be transformed into different phosphino- or arene-ruthenium complexes. The number of acetonitrile substitutions was controlled by temperature as room temperature promoted only one substitution, while refluxing conditions resulted in three acetonitrile substitutions.

\subsection{Continuous Flow Conditions to Prepare Iron and} Ruthenium Complexes

The Renaud and Whitby groups transferred their microwave procedures (Scheme 59) into a flow process with minor adjustments of reacting temperatures and solvents (Scheme 100). Using this method, mixed phosphorus-nitrogen ferrocene complexes $\mathbf{2 2 4}$ were obtained from [FeCp(naphthalene) $]\left[\mathrm{PF}_{6}\right] \mathbf{2 2 3}$ in slightly lower yields than via microwave irradiation. ${ }^{166}$

Cationic cyclopentadienyl ruthenium complexes $[\mathbf{R u C p}]^{+}$ are highly versatile catalysts due to their high $\pi$-acidic character, their capacity to hold reversible redox cycles, and their large range of oxidation states and coordination geometries. For these reasons the Jamison group investigated the preparation of $\left[\mathrm{RuCp}(\mathrm{ACN})_{3}\right] \mathbf{P F}_{6} 372$ from $\left[\mathrm{RuCp}\left(\eta^{6}-\right.\right.$ $\left.\left.\mathrm{C}_{6} \mathrm{H}_{6}\right)\right] \mathbf{P F}_{6} 371$ via continuous flow photochemistry. ${ }^{22}$ Synthesis was performed using homemade equipment featuring a coil wrapped around a condenser and a $450 \mathrm{~W}$ Hg lamp (Scheme 101). Under these conditions, the catalyst
Scheme 100. Continuous Flow Process for the Synthesis of Phosphino Iron Complexes

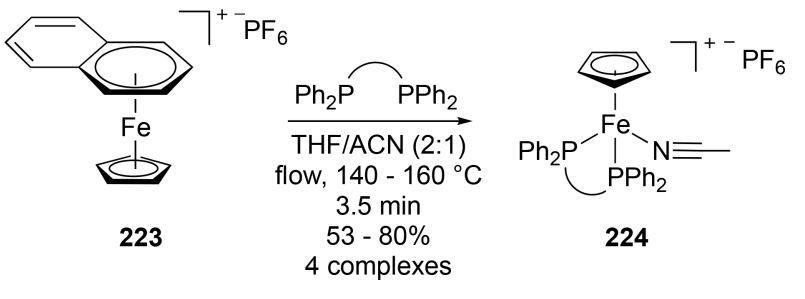

Scheme 101. Reactor for the Synthesis of

$\left[\mathrm{RuCp}(\mathrm{ACN})_{3}\right] \mathrm{PF}_{6}$

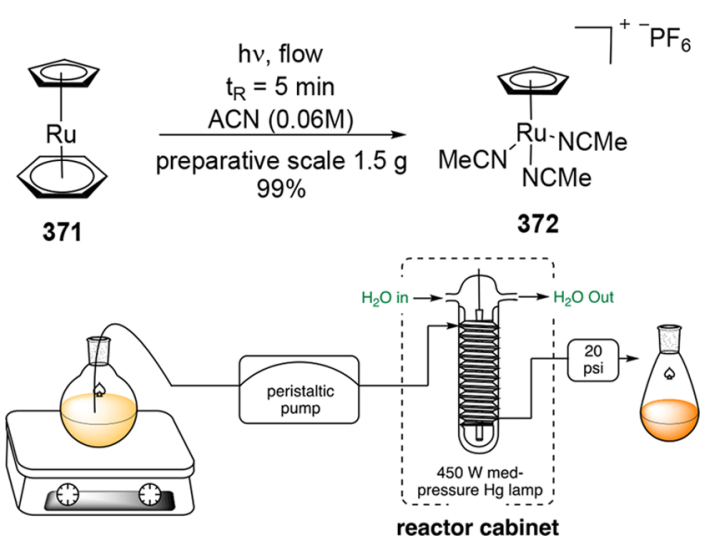

was formed in $>99 \%$ purity, and no additional purification was required. The ruthenium catalyst was produced with a throughput of $1.56 \mathrm{~g} / \mathrm{h}$ using a $5 \mathrm{~mL}$ reactor, which is 10 times higher than the reported batch process. Additionally, inter- and intramolecular ene-yne coupling reactions could be catalyzed by the species formed in situ during photolysis of $\mathbf{3 7 1}$ under continuous flow conditions. ${ }^{223}$ Interestingly, the catalyst could be quantitatively recovered at the end of the reaction.

\section{GROUP 9}

\subsection{Synthesis of Cobalt and Rhodium Complexes by Mechanochemistry}

Similarly to group 8 metal complexes, cobalt complexes exhibit promising activity against several human cancer cell lines, and several rhodium complexes exhibit oxygen-independent lightinduced anticancer activity. ${ }^{142}$ Cobalt complexes were also investigated for the inhibition of enzymes. ${ }^{6}$ As for iron(III) bisdicarbollyl complex 194, Borisov et al. described the mechanosynthesis of cobalt(III) bisdicarbollyl complex 375 in $1993 .{ }^{157}$ The reaction was performed in a stainless steel reactor that was charged with 15 steel balls ( $12 \mathrm{~mm}$ diameter), $\mathrm{CoCl}_{3}$, and $\mathrm{Tl}_{2} \mathrm{C}_{2} \mathrm{~B}_{9} \mathrm{H}_{11}$ 192. After $30 \mathrm{~min}$ of reaction at a frequency of $12 \mathrm{~Hz}$ and an amplitude of $11 \mathrm{~mm}$, the reaction

Scheme 99. Phototransformation of $\left[\mathrm{RuCp}\left(\eta^{6}\right.\right.$-benzene $\left.)\right] \mathrm{PF}_{6}$

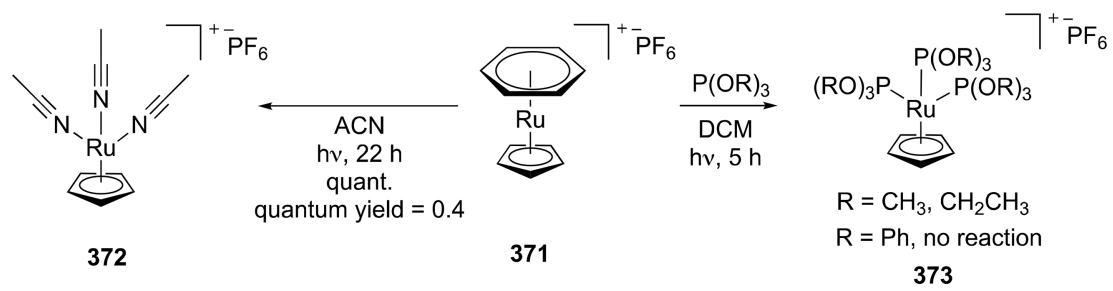


mixture was recovered and treated with an excess of $\mathrm{Me}_{4} \mathrm{NHSO}_{4}$ to isolate the desired cobalt complex 375 after filtration and recrystallization (Scheme 102). Importantly, the

Scheme 102. Mechanosynthesis of a Cobalt(III)Bisdicarbollyl Complex

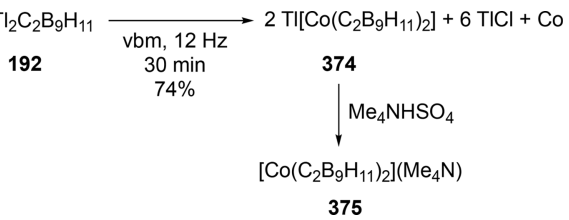

reaction could be performed at room temperature or at a maximum of $30-40{ }^{\circ} \mathrm{C}$ (if heating of the jar occurred during milling), whereas those described in solution had to be heated at $60{ }^{\circ} \mathrm{C}$.

Similarly to iron complexes, the zwitterionic $\left[\mathrm{Co}^{\mathrm{III}}\left(\eta^{5}\right.\right.$ $\left.\left.\mathrm{C}_{5} \mathrm{H}_{4} \mathrm{COOH}\right)\left(\eta^{5}-\mathrm{C}_{5} \mathrm{H}_{4} \mathrm{COO}\right)\right]$ complex reacted in the solid state under mechanical forces with numerous salts such as $\mathrm{KBr}$, $\mathrm{NH}_{4} \mathrm{PF}_{6}$, and $\mathrm{KPF}_{6}$ to produce the $\left[\mathrm{Co}{ }^{\mathrm{III}}\left(\boldsymbol{\eta}^{5}-\mathrm{C}_{5} \mathrm{H}_{4} \mathbf{C O O H}\right)-\right.$ $\left.\left(\boldsymbol{\eta}^{5}-\mathrm{C}_{5} \mathrm{H}_{4} \mathbf{C O O}\right)\right]_{2} \cdot \mathrm{C}^{+} \mathbf{A}^{-}\left(\mathrm{C}^{+}=\right.$cation, $\mathrm{K}^{+}, \mathrm{Rb}^{+}, \mathrm{Cs}^{+}$, and $\mathrm{NH}_{4}{ }^{+}$; $\mathrm{A}^{-}=$anion, $\mathrm{Cl}^{-}, \mathrm{Br}^{-}$, and $\mathrm{PF}_{6}^{-}$) complexes in quantitative yields. $^{224}$

In 1991, Ogawa et al. described the mechanochemical formation of $\left[\mathbf{C o}(\text { bipy) })_{3}\right] \mathbf{C l}_{2}$ complex $376 .{ }^{225}$ Dried cobalt(II) montmorillonites and bipy were ground in an agate mortar and pestle for a few minutes at room temperature under a dry air atmosphere (Scheme 103). Before grinding the solid mixture,

Scheme 103. Structure of Pyridine-Containing Cobalt Complexes

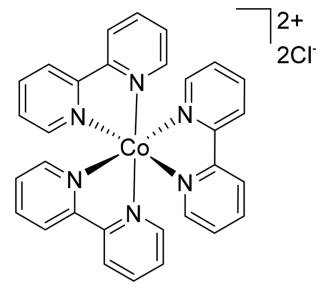

376

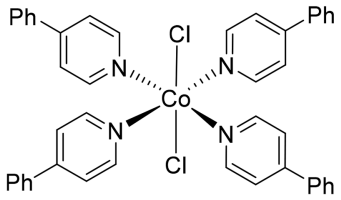

377
cobalt(II) montmorillonite was dried under two different conditions to vary the hydrated states of $\mathrm{Co}$ (II) interlayers (either at $120{ }^{\circ} \mathrm{C}$ for $2 \mathrm{~h}$ under vacuum or at $300{ }^{\circ} \mathrm{C}$ for $2 \mathrm{~h}$ under air). Whereas montmorillonite dried at $120^{\circ} \mathrm{C}$ led to the desired complex after grinding, when it was dried at $300{ }^{\circ} \mathrm{C}$ the expected complex was not produced.

Mechanochemical synthesis was also used to obtain the Werner complex $\left[\mathbf{C o C l}_{2}(4-\mathrm{PhPy})_{4}\right] \quad 377$ (4-PhPy $=4-$ phenylpyridine) by grinding the 4-PhPy ligand with $\mathrm{CoCl}_{2}$ (Scheme 103). ${ }^{226}$ Octahedral 0-D Werner complexes $\left[\mathrm{MX}_{2} \mathrm{~L}_{4}\right]$ are generally known for their ability to trap a variety of aromatic guests and have been employed in chromatography for the separation of aromatic compounds. ${ }^{227}$ Manual grinding of the ligand 4-PhPy with a mixture of $\mathrm{CoCl}_{2}$ and $\mathrm{NiCl}_{2}$ produced the solid solution $\left[\mathrm{Ni}_{0.5} \mathrm{Co}_{0.5} \mathrm{Cl}_{2}(4-\mathrm{PhPy})_{4}\right]$. Crystallization of a mixture of $\left[\mathrm{NiCl}_{2}(4-\mathrm{PhPy})_{4}\right]$ and $\left[\mathrm{CoCl}_{2}(4-\right.$ $\left.\mathrm{PhPy}_{4}\right] 377$ in $\mathrm{MeOH}$ did not result in the solid solution but a nonhomogeneous product (mixture of $\left[\mathbf{M C l}_{2}(4-\mathrm{PhPy})_{4}\right] ; \mathrm{M}$ $=\mathrm{Co}, \mathrm{Ni}$ ) composed of $\mathrm{MeOH}$ solvates of the $\mathrm{Ni}$ and $\mathrm{Co}$ complexes, showing that the solid solution can only be obtained by grinding.

Two mechanochemical methods were developed to prepare $\left[\mathrm{CoCl}_{2}(\mathrm{CEAP})_{2}\right] 381$ (CEAP $=4$ - $[N, N$-bis $(2$-cyanoethyl $)-$ amino]pyridine, 378). ${ }^{228} \mathrm{CEAP}$ and $\mathrm{CoCl}_{2} \cdot 6 \mathrm{H}_{2} \mathrm{O}$ (2:1 ratio) or $[\mathrm{CEAPH}]_{2}\left[\mathrm{CoCl}_{4}\right] 380$ and $\mathrm{KOH}$ were ground in an agate mortar and pestle for 15 min to produce the same complex 381. Notably, the hydrogen-bonded salt $\mathbf{3 8 0}$ that was used in the second method could also be synthesized mechanochemically by grinding CEAPH 379 and $\mathrm{CoCl}_{2} \cdot 6 \mathrm{H}_{2} \mathrm{O}$ for $15 \mathrm{~min}$ (Scheme 104). The products could be synthesized using classical solvothermal conditions, but longer reaction times were required to reach completion.

Similarly to the ruthenium complexes described above (Scheme 54), $\left[\mathbf{C o}(\mathbf{H b i i m})_{3}\right] \cdot 3 \mathbf{H}_{2} \mathbf{O}$ was synthesized in $84 \%$ yield by grinding with ammonium acetate and $\mathrm{H}_{2} \mathrm{O}_{2}$ in a mortar and pestle for $25 \mathrm{~min}$.

In 1997, the synthesis of dichlorobis(4-toluidine) cobalt(II) was reported by grinding $\mathrm{CoCl}_{2} \cdot 6 \mathrm{H}_{2} \mathrm{O}$ and 4-toluidine at $1: 2$ stoichiometric ratio at room temperature. The complex was obtained in $95 \%$ yield after 15 min of grinding. ${ }^{229}$ Similarly, the Liu group used various anilines, ground with $\mathrm{CoCl}_{2} \cdot 6 \mathrm{H}_{2} \mathrm{O}$, to form seven cobalt-amine complexes with the general formula $\left[\mathrm{CoCl}_{2}\left(\mathrm{H}_{2} \mathrm{O}\right)_{2}\left(\mathrm{ArNH}_{2}\right)_{2}\right]\left(\mathbf{3 8 2}\right.$, Scheme 105). ${ }^{230}$ The reactions were efficient with anilines containing an electrondonating group on the phenyl ring and led to the complexes quickly and in quantitative yields. On the contrary, when a moderate $\left(-\mathrm{COCH}_{3},-\mathrm{CO}_{2} \mathrm{C}_{2} \mathrm{H}_{5}\right)$ or strong $\left(-\mathrm{CN},-\mathrm{NO}_{2}\right)$ electron-withdrawing group was present on the aniline, the

\section{Scheme 104. Synthesis of Co-CEAP Complexes Using Mortar and Pestle}

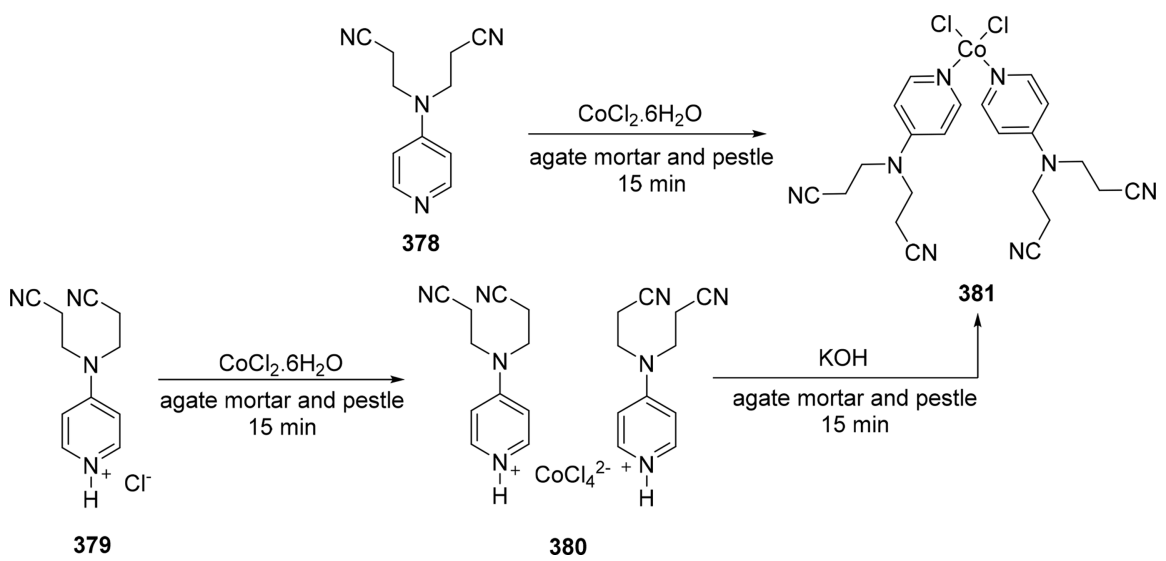


Scheme 105. Mechanochemical Synthesis of Cobalt-Amine Complexes

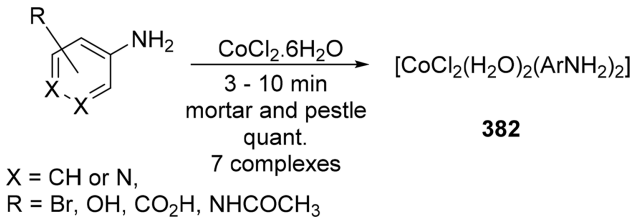

reactions were completely inhibited. Additionally, the use of ortho-substituted aromatic amines or the use of an extra aromatic ring on the aniline did not provide the desired complexes after mechanochemical treatment. Interestingly, the complexes were stable to air when stored at room temperature but were transformed back into the corresponding starting reactants when dissolved in a solvent.

Besides, cobalt complexes $\mathbf{3 8 4}$ were obtained by grinding diimines $\mathbf{3 8 3}$ with Co(II) sources using a Retsch MM400 mill at $30 \mathrm{~Hz}$ (Scheme 106). ${ }^{231}$ The reaction conditions were first

Scheme 106. Mechanochemical Synthesis of DiimineCobalt Complexes

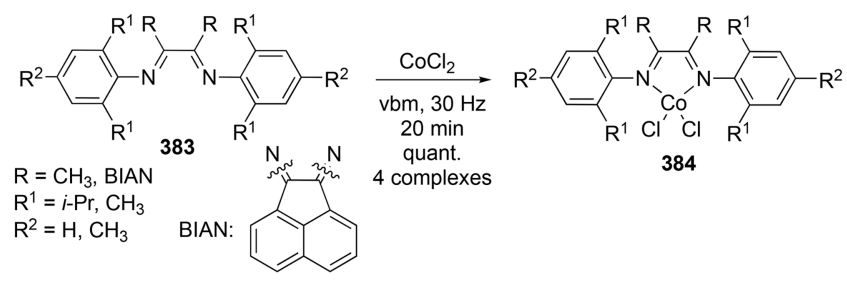

developed for the synthesis of $\mathrm{Ni}(\mathrm{II})$ complexes and then applied to the synthesis of four cobalt analogues. After only 20 min of grinding, the complexes were recovered in quantitative yields.

As for manganese(0) (Scheme 36), milling cobalt $(0)$ powder with an ortho-quinone $\mathbf{1 5 1}$ and pyridine led to the formation of mononuclear cobalt complex $\left[\mathrm{Co}(\mathrm{SQ})_{2}(\mathrm{Py})_{2}\right]$ 385 using solid-state metal oxidation and direct coordination of the in situ formed ligand to the metal (Scheme 107). ${ }^{124}$

Cobalt complex 387 featuring a Schiff base ligand derived from 1-[(2-biphenylamino)methylen]naphtalen-2(1H)-one was synthesized in quantitative yield by grinding ligand precursor 386 with $\left[\mathrm{Co}(\mathrm{OAc})_{2}\right] \cdot 4 \mathrm{H}_{2} \mathrm{O}$ and triethylamine followed by annealing at $160{ }^{\circ} \mathrm{C}$ (Scheme 108). ${ }^{232}$ Grinding was operated at $25 \mathrm{~Hz}$ for $50 \mathrm{~min}$ in a $10 \mathrm{~mL}$ stainless steel jar using a vibratory ball-mill Retsch MM200. Only a partial reaction was obtained by neat grinding. The course of the reaction differed remarkably and advantageously when using a liquid assistant. While the use of acetonitrile and $\mathrm{MeOH}$
Scheme 108. Mechanosynthesis of Schiff Base Cobalt Complexes

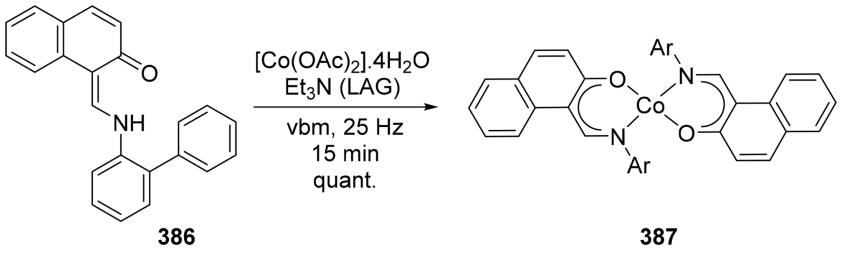

produced mixtures composed of solvates, trimethylamine led to the acetic solvate as the major product. Annealing at $160{ }^{\circ} \mathrm{C}$ enabled the removal of the acetic acid molecule to provide the expected cobalt complex. When synthesized in solution, complex 387 was isolated in only $62 \%$ yield.

Hernández and Bolm have developed the first mechanosynthesis of rhodium complexes. ${ }^{233}$ Indeed, $\left[\mathbf{R h C l}_{2} \mathbf{C p}^{*}\right]_{2}$ complex 388 was synthesized by grinding $\left[\mathbf{R h C l}_{3}\right]\left(\mathbf{H}_{2} \mathbf{O}\right)_{n}$ and $\mathrm{Cp} * \mathrm{H}$. Different ball mills (planetary or vibratory) and types of milling material (stainless steel, zirconium oxide, and tungsten carbide) were screened. The best results were obtained when using a planetary ball mill along with a tungsten carbide jar agitated at $800 \mathrm{rpm}$ for $3 \mathrm{~h}$, while $\mathrm{MeOH}$ was used as the liquid grinding assistant (Scheme 109).

Scheme 109. Mechanosynthesis of $\left[\mathrm{RhCl}_{2} \mathrm{Cp}^{*}\right]_{2}$

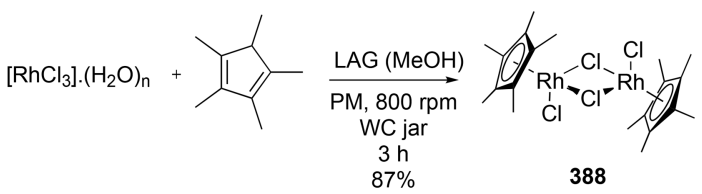

Complex 388 could also be synthesized in neat conditions or by using EtOH as an assistant but in lower yields. To confirm that the formation of the rhodium complex occurred during the milling process and not during the analysis or the workup procedure, a mixture of $\left[\mathbf{R h C l}_{3}\right] \cdot\left(\mathbf{H}_{2} \mathbf{O}\right)_{n}, \mathrm{Cp} * \mathrm{H}$, and $\mathrm{MeOH}$ was stirred at room temperature in $\mathrm{CDCl}_{3}$. After $6 \mathrm{~h}$ of agitation, no dimer was detected, demonstrating the efficiency of the milling process. Rhodium complex $\mathbf{3 8 8}$ was then used as a catalyst for the mechanochemical halogenation of 2phenylpyridine using NBS or NIS ( $\mathrm{N}$-bromosuccinimide and $\mathrm{N}$-iodosuccinimide). Under these conditions, reactions occurred without heating and in short reaction times (3-8 h).

The same group developed the synthesis of Wilkinson's catalyst $\left[\mathbf{R h C l}\left(\mathbf{P P h}_{3}\right)_{3}\right] \mathbf{3 8 9}$ in the ball mill in 2018 (Scheme $110) .{ }^{234}$ This approach was revealed to be highly interesting since ball milling under solvent-free conditions, or even with an alcoholic liquid grinding assistant, allowed the isolation of an elusive polymorphic orange form, which was only observed via

Scheme 107. Use of Cobalt Powder for the Synthesis of the $\left[\mathrm{Co}(\mathrm{SQ})_{2}(\mathrm{Py})_{2}\right]$ Complex

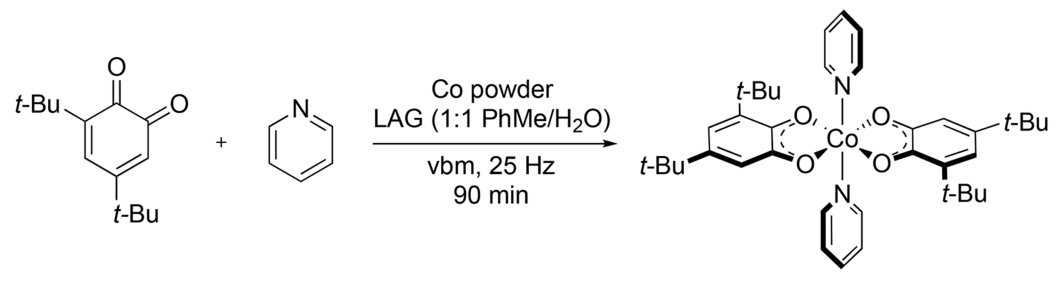


Scheme 110. Mechanochemical Isolation of the Orange Polymorph of Wilkinson's Catalyst

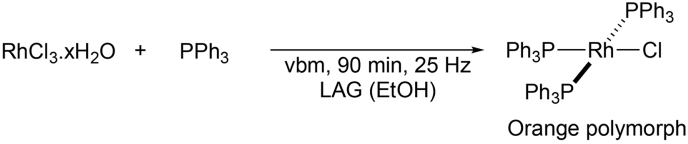

$$
\begin{aligned}
& 389
\end{aligned}
$$

classical solution synthesis when reduced quantities of solvent were used. The complex polymorphism was confirmed by PXRD and ${ }^{31} \mathrm{P}$ solid-state NMR. The two polymorphs of Wilkinson's catalyst were evaluated in the dehydrocoupling of amine borane and further hydrogenation of olefins. In the aformentioned reaction, the efficiency was dependent on the polymorph used, indicating the importance of isolating both polymorphs.

\subsection{Procedures Using Microwave Irradiation}

As for chromium complexes (see section 6.2), the Jain and Mishra groups reported the microwave-assisted synthesis of numerous cobalt(II) complexes with Schiff bases as ligands between 2010 and 2016. Syntheses were performed in an open glass vessel using a modified open microwave (model 2001 ETB) equipped with a rotating tray. The microwave delivered an $800 \mathrm{~W}$ energy output, while the irradiation frequency was fixed at $2450 \mathrm{MHz}$. To control the temperature, microwave reactions were performed using on/off cycles. $\left[\mathrm{Co}(84)_{2}\right]$. $\mathbf{2 H}_{2} \mathbf{O},{ }^{97}\left[\mathrm{Co}(\mathbf{8 3})\left(\mathbf{H}_{2} \mathbf{O}\right)\right] \mathbf{C l},{ }^{97}\left[\mathrm{Co}(\mathbf{8 8})\left(\mathbf{H}_{2} \mathbf{O}\right)_{2}\right],{ }^{98}[\mathbf{C o}-$ $\left.(\mathbf{8 1})_{2}\left(\mathbf{H}_{2} \mathbf{O}\right)_{2}\right],{ }^{99}\left[\mathbf{C o}(\mathbf{8 2})_{2}\left(\mathbf{H}_{2} \mathbf{O}\right)_{2}\right],{ }^{99}\left[\mathbf{C o}(\mathbf{8 5})_{2}\left(\mathbf{H}_{2} \mathbf{O}\right)_{2}\right] \cdot$ $\mathbf{H}_{2} \mathbf{O},{ }^{100}\left[\mathrm{Co}(86)_{2}\left(\mathbf{H}_{2} \mathbf{O}\right)_{2}\right],{ }^{100}\left[\mathrm{Co}(78)_{2}\left(\mathbf{H}_{2} \mathbf{O}\right)_{2}\right],{ }^{96}[\mathrm{Co}-$ $\left.(\mathbf{8 0})_{2}\left(\mathrm{H}_{2} \mathrm{O}\right)_{2}\right],{ }^{96}\left[\mathrm{Co}(76)_{2}\left(\mathrm{H}_{2} \mathbf{O}\right)_{2}\right] \cdot \mathrm{Cl}_{2},{ }^{235}[\mathrm{Co}-$ $\left.(77)_{2} \mathrm{Cl}_{2}\right] \cdot 2 \mathrm{H}_{2} \mathrm{O},{ }^{235}\left[\mathrm{Co}(74)\left(\mathrm{H}_{2} \mathrm{O}\right)_{3}\right],{ }^{95}$ and $[\mathrm{Co}(75) \mathrm{Cl}] \cdot$ $\mathbf{2 H}_{\mathbf{2}} \mathbf{O}^{95}$ were synthesized as follows. Ligand and $\mathrm{CoCl}_{2}$. $6 \mathrm{H}_{2} \mathrm{O}$ were ground together in a stoichiometric ratio (1:1 or 2:1) before being transferred to a reactor with $3-5 \mathrm{~mL}$ of dry EtOH and heated under microwave irradiation for 6-8 $\mathrm{min}$ (Table 6, entries 1 to 13, see Figure 1 for ligand structures).

Table 6. Microwave versus Conventional Heating for the Synthesis of Co-Schiff Base Complexes ${ }^{a}$

\begin{tabular}{|c|c|c|c|c|c|c|}
\hline \multirow[b]{2}{*}{ entry } & \multirow[b]{2}{*}{ cobalt complex } & \multicolumn{2}{|c|}{$\begin{array}{l}\text { microwave } \\
\text { synthesis }\end{array}$} & \multicolumn{2}{|c|}{$\begin{array}{c}\text { conventional } \\
\text { heating }\end{array}$} & \multirow[b]{2}{*}{ ref } \\
\hline & & time & $\begin{array}{c}\text { yield } \\
(\%)\end{array}$ & $\begin{array}{l}\text { time } \\
(\mathrm{h})\end{array}$ & $\begin{array}{c}\text { yield } \\
(\%)\end{array}$ & \\
\hline 1 & {$\left[\mathrm{Co}(84)_{2}\right] \cdot 2 \mathrm{H}_{2} \mathrm{O}$} & $7 \mathrm{~min}$ & 79 & 7 & 64 & 97 \\
\hline 2 & {$\left[\mathrm{Co}(83)\left(\mathrm{H}_{2} \mathrm{O}\right)\right] \mathrm{Cl}$} & $8 \mathrm{~min}$ & 78 & 7 & 64 & 97 \\
\hline 3 & {$\left[\mathrm{Co}(88)\left(\mathrm{H}_{2} \mathrm{O}\right)_{2}\right]$} & $8 \min$ & 84 & 8.5 & 64 & 98 \\
\hline 4 & {$\left[\mathrm{Co}(81)_{2}\left(\mathrm{H}_{2} \mathrm{O}\right)_{2}\right]$} & $7 \mathrm{~min}$ & 85 & 7 & 67 & 99 \\
\hline 5 & {$\left[\mathrm{Co}(82)_{2}\left(\mathrm{H}_{2} \mathrm{O}\right)_{2}\right]$} & $8 \mathrm{~min}$ & 82 & 7 & 68 & 99 \\
\hline 6 & {$\left[\mathrm{Co}(85)_{2}\left(\mathrm{H}_{2} \mathrm{O}\right)_{2}\right] \cdot \mathrm{H}_{2} \mathrm{O}$} & $8 \mathrm{~min}$ & 82 & 8 & 69 & 100 \\
\hline 7 & {$\left[\mathrm{Co}(86)_{2}\left(\mathrm{H}_{2} \mathrm{O}\right)_{2}\right]$} & $8 \mathrm{~min}$ & 81 & 8 & 66 & 100 \\
\hline 8 & {$\left[\mathrm{Co}(78)_{2}\left(\mathrm{H}_{2} \mathrm{O}\right)_{2}\right]$} & $8 \mathrm{~min}$ & 81 & 7 & 68 & 96 \\
\hline 9 & {$\left[\mathrm{Co}(80)_{2}\left(\mathrm{H}_{2} \mathrm{O}\right)_{2}\right]$} & $8 \mathrm{~min}$ & 82 & 6.5 & 62 & 96 \\
\hline 10 & {$\left[\mathrm{Co}(76)_{2}\left(\mathrm{H}_{2} \mathrm{O}\right)_{2}\right] \cdot \mathrm{Cl}_{2}$} & $7 \mathrm{~min}$ & 82 & 7 & 68 & 235 \\
\hline 11 & {$\left[\mathrm{Co}(77)_{2} \mathrm{Cl}_{2}\right] \cdot 2 \mathrm{H}_{2} \mathrm{O}$} & $8 \mathrm{~min}$ & 81 & 8 & 66 & 235 \\
\hline 12 & {$\left[\mathrm{Co}(74)\left(\mathrm{H}_{2} \mathrm{O}\right)_{3}\right]$} & $7 \mathrm{~min}$ & 81 & 7 & 68 & 95 \\
\hline 13 & {$[\mathrm{Co}(75) \mathrm{Cl}] \cdot 2 \mathrm{H}_{2} \mathrm{O}$} & $7 \mathrm{~min}$ & 79 & 8 & 65 & 95 \\
\hline 14 & {$[\mathrm{Co}(87)]$} & $1 \mathrm{~min}$ & 85 & 5 & 72 & 135 \\
\hline 15 & {$\left[\mathrm{Co}(79)_{2}\right]$} & $25 \mathrm{~s}$ & 95 & 5 & 83 & 133 \\
\hline 16 & {$[\mathrm{Co}(71)] \cdot \mathrm{H}_{2} \mathrm{O}$} & $9 \min$ & 85 & 8 & 70 & 134 \\
\hline
\end{tabular}

${ }^{a}$ See Figure 1 for the structure of ligands.
The corresponding products were recovered in high yields ranging from 78 to $85 \%$. Microwave irradiation outperformed classical conditions, allowing for a reduction in time from hours to only a few minutes and an increase in yields from 64$69 \%$ to $78-85 \%$.

The work of Jain and Mishra followed related work from the Meshram group. Indeed, Meshram et al. reported a similar microwave-assisted synthesis of $[\mathbf{C o}(\mathbf{8 7})]^{135}$ and $[\mathbf{C o -}$ $\left.(79)_{2}\right]^{133}$ in 2009 (Table 6, entries 14 and 15). These complexes were obtained after microwave irradiation of an ethanolic $\left[\mathrm{Co}(\mathrm{OAc})_{2}\right] \cdot\left(\mathrm{H}_{2} \mathrm{O}\right)_{n}$ solution and the corresponding ligand for less than a minute in a domestic oven, providing the products in excellent yields. A similar procedure was also described in 2014 for the synthesis of complex $[\mathbf{C o}(71)] \cdot \mathbf{H}_{2} \mathrm{O}$ (Table 6, entry 16). ${ }^{134}$ Only 9 min under microwave irradiation was necessary to obtain complete conversion (compared to $8 \mathrm{~h}$ in solution), producing the complex in $85 \%$ yield.

In 2016, a microwave-assisted synthesis of three macrocyclic Co(II) complexes 392 was described (Scheme 111). ${ }^{236}$

Scheme 111. Microwave Synthesis of the Macrocyclic Co(II) Complex 392
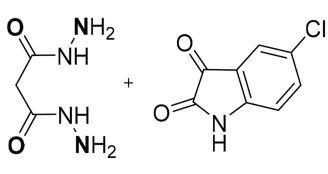

390

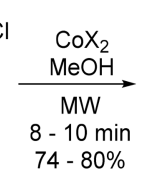

391

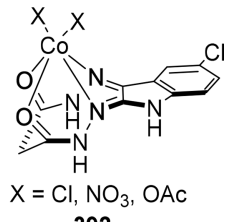

392
Malonic acid dihydrazide 390, 5-chloroisatin 391, and $\mathrm{CoX}_{2}$ $\left(\mathrm{X}=\mathrm{Cl}, \mathrm{NO}_{3}\right.$ or $\left.\mathrm{OAc}\right)$ were mixed in a grinder in a 1:1:1 ratio before being irradiated in a microwave synthesizer for 8-10 min. The corresponding products were obtained in yields between 74 and $80 \%$, higher in comparison to conventional reflux conditions.

Similarly to Schiff base complexes, cobalt complex 394 featuring a luminol derivative ligand could be obtained in $83 \%$ yield via microwave heating (Scheme 112). ${ }^{237}$ Notably, ligand 393 and the metal source were finely mixed using a mortar and pestle before being placed under microwave irradiation in the absence of a solvent.

Scheme 112. Microwave Synthesis of a Cobalt Complex with Luminol Derivative
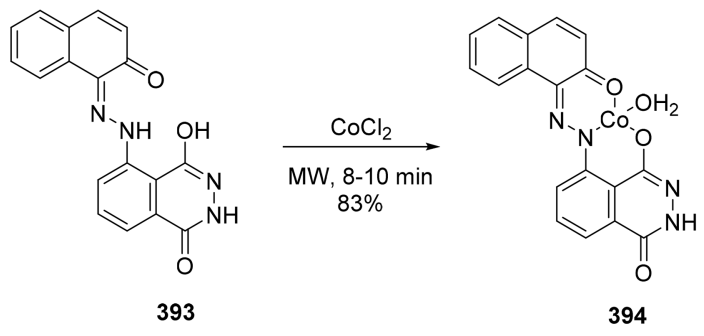

As for the synthesis of vanadium (see section 5.2) and chromium (see section 6.2) complexes, Alias et al. described the microwave synthesis of a mixed phenanthroline and 2acetamide benzothiazole cobalt complex 395 in 2015 (Scheme 113). ${ }^{83}$ The product was recovered in $95 \%$ yield after only 1 min of microwave heating. 
Scheme 113. Microwave-Assisted Synthesis of a Mixed Ligand Cobalt Complex

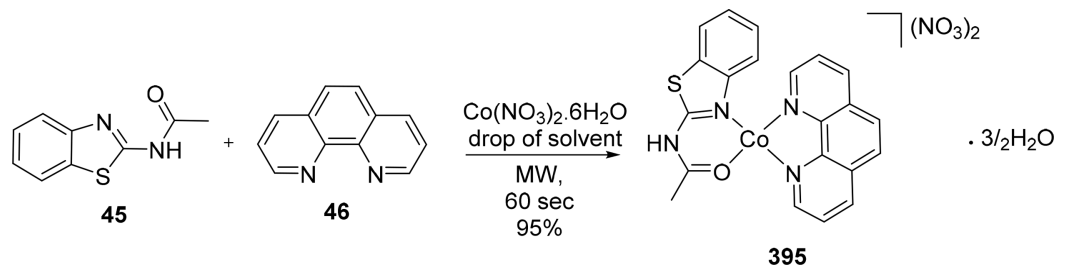

Scheme 114. Microwave-Assisted Cycloaddition for the Formation of Cobalt(III)-Tetrazolate Complexes

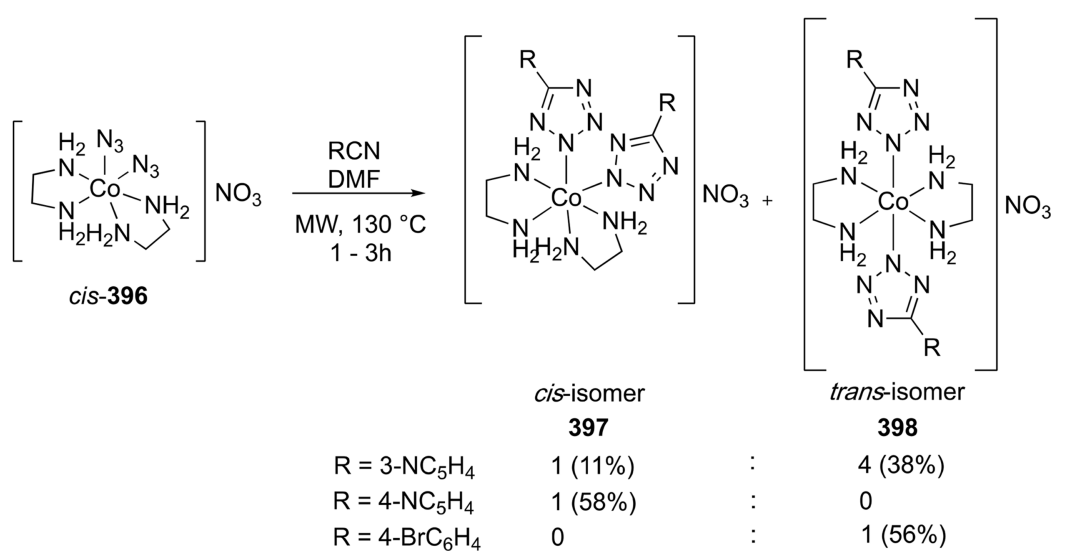

Microwave-assisted 1,3-dipolar cycloaddition reaction of cis$\left[\mathrm{Co}\left(\mathrm{N}_{3}\right)_{2}(\mathrm{en})_{2}\right] \mathrm{NO}_{3} 396$ (en = 1,2-ethylenediamine) with different organonitriles ( $\mathrm{R}-\mathrm{CN}$ ) produced the bistetrazolate $\left[\mathrm{Co}\left(\mathrm{N}_{4} \mathrm{CR}\right)_{2}(\mathrm{en})_{2}\right]\left(\mathrm{NO}_{3}\right) 397$ and 398 in $1-3 \mathrm{~h}$ at $130{ }^{\circ} \mathrm{C}$. $^{238}$ Interestingly, changing the nitrile $\left(\mathrm{R}=3-\mathrm{NC}_{5} \mathrm{H}_{4}, 4-\mathrm{NC}_{5} \mathrm{H}_{4}\right.$, and $4-\mathrm{BrC}_{6} \mathrm{H}_{4}$ ) gave different ratios of isomers (Scheme 114). Starting from trans- $\left[\mathrm{Co}\left(\mathrm{N}_{3}\right)_{2}(\mathrm{en})_{2}\right] \mathbf{C l O}_{4}$, cycloaddition reaction with the same organonitriles using microwave irradiation unexpectedly produced a mixture of both isomers with the cisisomer as the major product.

Taylor et al. described the microwave-assisted synthesis of planar chiral cobalt metallocenes (Scheme 115). ${ }^{239}$ Five-

Scheme 115. Microwave-Assisted Synthesis of Planar Chiral Cobalt Metallocenes

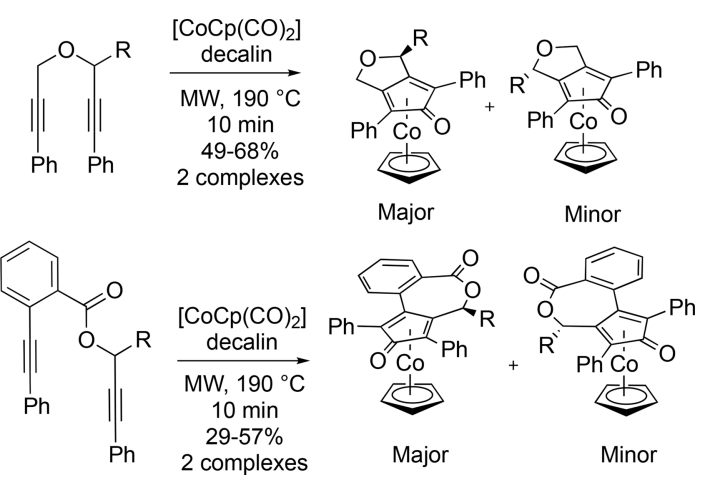

membered ring cyclopentadienone complexes 400 and 401 were obtained from ether-linked diynes 399. Similarly, esterlinked diynes $\mathbf{4 0 2}$ produced 7-membered ring cyclopentadienone complexes 403 and 404. Compounds 400 and 401 were isolated in similar yields, whether using classical reflux condition or microwave irradiation. Despite this, application of microwaves increased yields from $21-33 \%$ to $29-57 \%$ in the synthesis of $\mathbf{4 0 3}$ and $\mathbf{4 0 4}$ (Scheme 115). For all complexes, only $10 \mathrm{~min}$ was necessary to complete the reaction, whereas $18 \mathrm{~h}$ was required under classical reflux conditions. Both isomers could be separated by column chromatography or by recrystallization and were characterized by X-ray diffraction.

Harcourt et al. developed a similar approach using microwave irradiation to synthesize cyclopentadienyl-cobalt sandwich complexes $405-406$ by intermolecular cyclization of diarylacetylene (Scheme 116). ${ }^{240}$ In comparison with the

Scheme 116. Microwave-Assisted Synthesis of Cobalt Sandwich Complexes

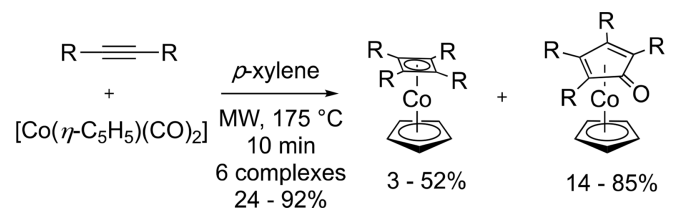

$$
\begin{aligned}
& \mathrm{R}=\text { phenyl, pyridine, napthyl, thienyl } \\
& 405
\end{aligned}
$$

traditional thermal approach, the method reduced the reaction times and increased the yields. In the case of bulky diarylacetylene compounds, the microwave approach gave access to complexes, which could not be obtained under classical heating conditions. Interestingly, a first one-pot/twostep synthesis was developed to form cobalt complexes. The synthesis started with a Sonogashira coupling to generate the diphenylacetylene in situ, followed by the cyclization-metalation to obtain the desired product. The proof of concept was established with one example, yet with lower yield compared to the step-by-step synthesis.

The Aubert and Gandon group described an improved protocol for the synthesis of sandwich cobalt complexes 409 in 2012. In contrast with preceding studies, microwave-assisted synthesis proved to be highly chemoselective and yielded pure complexes in almost all cases. ${ }^{241}$ The use of the nonclassical cobalt source 408 instead of $\left[\mathrm{CoCp}(\mathrm{CO})_{2}\right]$ or $[\mathrm{CoCp}$ - 
$\left.\left(\mathrm{C}_{2} \mathrm{H}_{4}\right)_{2}\right]$ was shown to inhibit the formation of other insertion products (cyclopentadienone or cyclohexadiene). The procedure was applied to the synthesis of numerous complexes starting from different alkynes: diarylacetylene (Scheme 117),

Scheme 117. Microwave Synthesis of $[\mathrm{CoCpCb}]$ Complexes
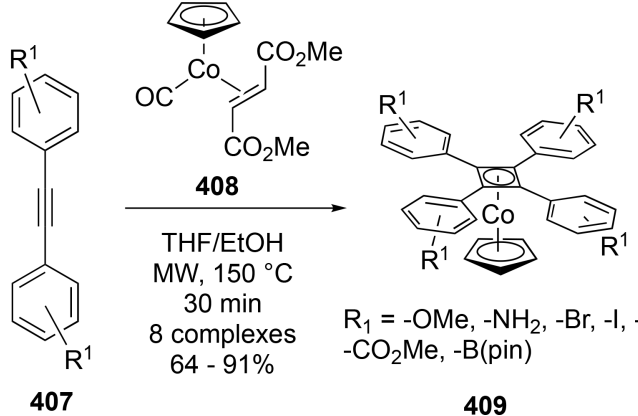

$\mathrm{R}_{1}=-\mathrm{OMe},-\mathrm{NH}_{2},-\mathrm{Br},-\mathrm{I},-\mathrm{C}_{4} \mathrm{H}_{9}$ $\mathrm{CO}_{2} \mathrm{Me},-\mathrm{B}$ (pin)

\section{9}

thienylalkynes, unsymmetrical diarylalkyne, but also diynes. No product was formed when diphenyl acetylene with ortho substituent was employed as a reactant. Due to the presence of functional groups on the diphenylacetylene, several modifications could be applied to the cobalt complexes, such as crosscoupling (when $\mathrm{R}^{1}=\mathrm{Br}$, I, or $\mathrm{B}($ pin $)$ ) or saponification and reduction into alcohol (when $\mathrm{R}^{1}=\mathrm{CO}_{2} \mathrm{Me}$ ).

Microwave-assisted heating also proved beneficial for the synthesis of rhodium complexes. For instance, $\left[\mathbf{R h}_{2}(\mathbf{p f m})_{4}\right]$ complex 411 (pfm = perfluorobutyramide, 410) was initially prepared by heating a solution of perfluorobutyramide and rhodium acetate in chlorobenzene at reflux for $60 \mathrm{~h}$ using a Soxhlet extraction apparatus. To develop an easier and faster method, the same reaction was performed using a Biotage Initiator operated at $205 \mathrm{~W}$ (Scheme 118). ${ }^{242}$ After $30 \mathrm{~min}$ of

Scheme 118. Microwave Preparation of Rhodium

Perfluorobutyramide

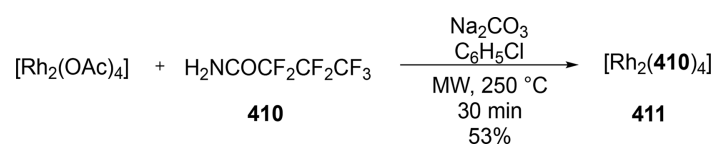

microwave-assisted heating at $250{ }^{\circ} \mathrm{C}, \mathbf{4 1 1}$ was isolated in $53 \%$ yield. It was then used as catalyst in the trichloroethoxysulfonyl, nosyl, and tosyl aziridination of olefins (nosyl = 4nitrobenzene-1-sulfonyl; tosyl = 4-methylbenzene-1-sulfonyl).

Classical syntheses of the cis- $\left[\mathrm{Rh}(\text { bipy })_{2} \mathrm{X}_{2}\right]^{+} 412(\mathrm{X}=\mathrm{Cl}$, $\mathrm{Br}, \mathrm{I})$ complex gave reasonable yields $(70-90 \%)$ but were limited by the tedious purification required to enable the photophysical study of these complexes. This is mainly due to the formation of $\left[\mathbf{R h}(\right.$ bipy $\left.) \mathbf{X}_{4}\right]$, trans- $\left[\mathbf{R h}(\text { bipy })_{2} \mathbf{X}_{2}\right]^{+}$, and $\left[\mathbf{R h}(\text { bipy })_{3}\right]^{3+}$ as these byproducts can be difficult to remove, in particular the tris complex, which is highly light-emissive. To develop improved protocols to produce cis-412, the Megehee group reported syntheses via conventional and microwave heating (Scheme 119). ${ }^{243}$ If yields were comparable employing both techniques, microwave reactions required shorter reaction times (1.25-4 min compared to $20-65 \mathrm{~min}$ ), yet the reaction temperature was more difficult to control, sometimes leading to the formation of the undesirable and insoluble $\mathrm{Rh}_{2} \mathrm{O}_{3}$. The complexes were easily recovered by precipitation using $\mathrm{KPF}_{6}$. Notably, the authors highlighted the fact that a complete
Scheme 119. Microwave-Assisted Synthesis of $\left[\mathrm{Rh}(\text { bipy })_{2} \mathrm{X}_{2}\right]^{+}$

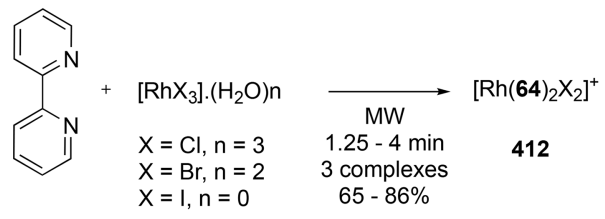

solubilization of the reactants before heating the reaction mixture was a key to obtain pure complexes.

Since the clinical success of platinum complexes for the development of novel antitumor agents, there has been an increasing demand for the development of metallodrugs for these applications. Due to the main disadvantages of these Ptcontaining drugs (low efficacy against widespread tumors, intrinsic and acquired resistance of neoplasms, and severe side effects accompanying the therapy), different metallodrugs have been developed. In this context, a new microwave synthesis of antitumor pentamethylcyclopentadienyl rhodium complexes of maltol (414) and allomaltol (415) was developed. Both complexes were recovered in $71 \%$ yield and after only a few seconds of heating (Scheme 120). ${ }^{244}$ Contrastingly, $18 \mathrm{~h}$ at

Scheme 120. Microwave-Assisted Synthesis of

Pentamethylcyclopentadienyl Rhodium Complexes Derived from Maltol and Allomaltol

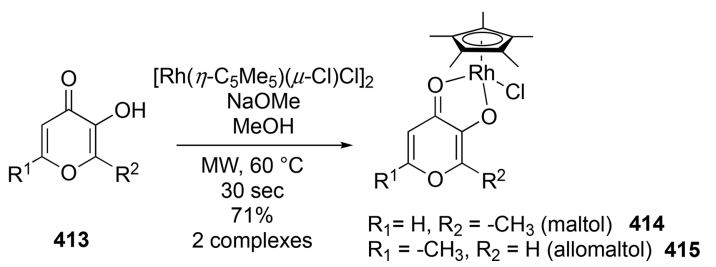

room temperature was necessary to synthesize 415 in $45 \%$ yield from allomaltol. Unfortunately, 414 and $\mathbf{4 1 5}$ were found to exhibit moderate to low cytotoxicity with $\mathrm{IC}_{50}$ (half maximal inhibitory concentration) values in the range of $100-300 \mu \mathrm{M}$.

As for rhenium (see section 7.2) and ruthenium (see Scheme 78$)$ complexes, $[\mathbf{R h C l}(\mathbf{C O D})]_{2}$ was produced using a modified commercially available microwave oven. ${ }^{125} \mathrm{RhCl}_{3}$. $x \mathrm{H}_{2} \mathrm{O}$ and cyclooctadiene in a $\mathrm{EtOH} /$ water (5:1) mixture were heated for $25 \mathrm{~min}$ to provide the complex in $87 \%$ yield. This reaction time could be reduced to only $1 \mathrm{~min}$ when the reaction was performed in an autoclave placed inside the microwave apparatus. Contrastingly, conventional refluxing conditions required $18 \mathrm{~h}$ of heating to produce the complex in good yield. Although the reaction time was considerably reduced using the microwave reflux procedure, the temperature was difficult to control. Two years later the same group published a slightly modified procedure to obtain $[\mathbf{R h C l}$ (COD) $]_{2}$ in $84 \%$ yield after $30 \mathrm{~s}$ at $140{ }^{\circ} \mathrm{C}$ under microwave irradiation. $^{200}$

The very first report on microwave-assisted synthesis of organometallic complexes was published in 1989 by Baghurst and Mingos. This publication described the synthesis of diolefin-rhodium(I) complexes by microwave-assisted heating of a mixture composed of an olefin and $\mathrm{RhCl}_{3} \cdot x \mathrm{H}_{2} \mathrm{O}$ in an alcoholic solvent $(\mathrm{MeOH}$ or $\mathrm{EtOH}) .{ }^{245}$ One minute of heating was sufficient to provide the rhodium complexes in more than $62-91 \%$ yield. Interestingly, the use of freshly distilled 
cyclopentadiene resulted in the sandwich cation $\left[\mathbf{R h}(\mathbf{C p})_{2}\right]^{+}$ instead of the chloro-bridged dimer $[\mathbf{R h}(\text { olefin }) \mathbf{C l}]_{2}$ obtained in other cases $\left(\mathrm{C}_{8} \mathrm{H}_{12}\right.$ or norbornadiene). The procedure was also applied to one example of the diolefin-iridium complex. $\left[\operatorname{Ir}\left(\mathbf{C}_{8} \mathbf{H}_{12}\right) \mathbf{C l}\right]_{2}$ was recovered in $72 \%$ yield after $45 \mathrm{~s}$ of heating instead of several hours in classical refluxing conditions. $^{245}$

As for $\left[\mathbf{R u C l}_{2} \text { (cyclopentadienyl) }\right]_{2}$ (see section 8.2 , Scheme 78 ), the Severin group reported a microwave-assisted synthesis of $\left[\mathrm{RhCl}_{2} \text { (cyclopentadienyl) }\right]_{2}$ and $\left[\mathrm{IrCl}_{2}\right.$ (cyclopentadienyl $)]_{2}$ (cyclopentadienyl $=$ tetramethylcyclopentadienyl and pentamethylcyclopentadienyl $\left.\mathrm{Cp}^{*}\right) .{ }^{205}$ Although conventional refluxing conditions required very long reaction times (21-48 $\mathrm{h}$ for $\left[\mathrm{RhCl}_{2} \mathbf{C p}^{*}\right]_{2}$ and $36-48 \mathrm{~h}$ for $\left.\left[\mathrm{IrCl}_{2} \mathbf{C p}^{*}\right]_{2}\right)$, heating a methanolic solution of $\left[\mathrm{RhCl}_{3}\right] \cdot\left(\mathrm{H}_{2} \mathrm{O}\right)_{n}$ or $\left[\mathrm{IrCl}_{3}\right]$. $\left(\mathrm{H}_{2} \mathrm{O}\right)_{n}$ with pentamethylcyclopentadiene under microwave irradiation led to the formation of the corresponding dimeric complexes in only $3 \mathrm{~min}$. Complexes were obtained in excellent yields ( $88 \%$ yield for the rhodium complex and $87 \%$ for the iridium complex). Tetramethylcyclopentadienyl complexes of rhodium and iridium have received less attention in the literature. Despite this, by applying the same procedure they could be obtained in good yields (79\% and 67\%, respectively).

As for ruthenium and osmium (see section 8.2) complexes, Schmidlehner et al. reported the synthesis of two different types of rhodium complexes by microwave irradiation in 2014 (Scheme 121). ${ }^{204}$ Heating a solution of $\left[\mathrm{Rh}\left(\mathrm{Cp}^{*}\right) \mathrm{Cl}_{2}\right]_{2}$ and

Scheme 121. Microwave Synthesis of Azole-Metal Complexes

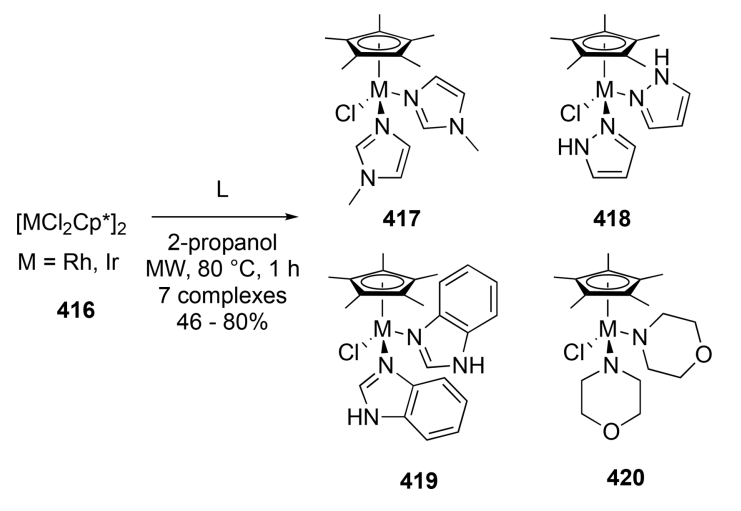

methylimidazole or pyrazole in 2-propanol at $80{ }^{\circ} \mathrm{C}$ provided the cationic $\left[\mathbf{R h}\left(\mathbf{C p}^{*}\right)(\text { azole })_{2} \mathbf{C l}\right] \mathbf{C l}$ complexes in only $1 \mathrm{~min}$ (417 and 418 ) in good yields ( $80 \%$ and $76 \%$, respectively). Similarly, heating the same starting materials for the same time with benzimidazole or morpholine at $80{ }^{\circ} \mathrm{C}$ resulted in the neutral $\left[\mathrm{Rh}\left(\mathrm{Cp}^{*}\right)(\right.$ azole $\left.) \mathrm{Cl}_{2}\right]$ complexes $(419$ and 420$)$ in lower yields (52\% and 70\%). Subsequently, the same operating procedure was applied to synthesize three iridium complexes with methylimidazole, pyrazole, and morpholine. In 1-2 min, products were recovered in moderate yields $(62 \%, 46 \%$, and $46 \%$, respectively).

In an interesting approach, rhodium and iridium complexes were prepared with iodide ion binding as models for an application in biomolecule labeling with ${ }^{211}$ At as an iodide replacement. ${ }^{246}$ Microwave-assisted synthesis was investigated due to the rapid conversion of the metal source to the product (compared with classical conditions which takes 1-1.5 h), which is necessary for a radiopharmaceutical study. Rhodium and iridium complexes (421 and 422) were synthesized in good yields ( $90 \%$ and $83 \%$, respectively) after 35 min under microwave irradiation (Scheme 122).

Scheme 122. Microwave Synthesis of Potential Precursors for Astatine Radiopharmaceuticals

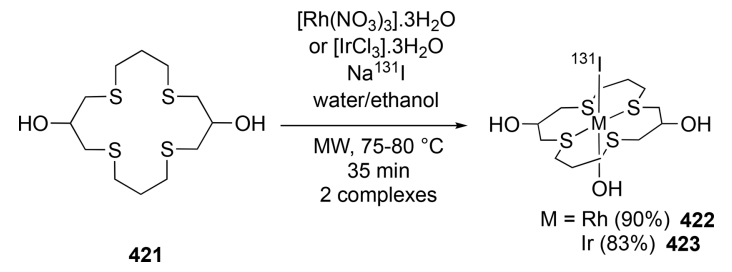

Furthermore, the $\left[\operatorname{Ir}\left(\mathrm{SC}\left(\mathrm{NH}_{2}\right)_{2}\right)_{2} \mathbf{C l}_{4}\right]\left[\operatorname{Ir}\left(\mathrm{SC}\left(\mathrm{NH}_{2}\right)_{2}\right)_{4} \mathrm{Cl}_{2}\right]$ complex was synthesized by reacting $\mathrm{K}_{3}\left[\mathrm{IrCl}_{6}\right]$ with thiocarbamide using a microwave oven at $800 \mathrm{~W}$ for 1 min. ${ }^{247}$ Although coordination compounds with thiourea or thiocarbamide are known in the literature, iridium complexes with thiocarbamide have not been adequately investigated. Under microwave irradiation, the iridium(III) chloride complex formed burgundy crystals. In contrast to using conventional heating, the synthesis led to a complex with the same composition but with a yellow color and a different crystallographic structure.

In 1990, Baghurst et al. reported the synthesis of two other iridium complexes via microwave irradiation. ${ }^{91}\left[\mathrm{IrCl}_{3}\right] \cdot\left(\mathbf{H}_{2} \mathbf{O}\right)_{n}$ and $\mathrm{PPh}_{3}$ were irradiated using a microwave oven (500-600 $\mathrm{W})$ in DMF for $45 \mathrm{~s}$ to give $\left[\operatorname{IrCl}(\mathbf{C O})\left(\mathbf{P P h}_{3}\right)_{2}\right]$ in $70 \%$ yield. The procedure was applied to classical reflux conditions and led to the formation of the same complex in $12 \mathrm{~h}$, which was, however, contaminated with $\left[\mathrm{IrCl}_{3}(\mathrm{CO})\left(\mathrm{PPh}_{3}\right)_{2}\right]$. To eliminate this oxidized byproduct, it was necessary to add zinc as a reducing agent to the reaction mixture. This addition was unnecessary when using microwave irradiation. In a similar method, $\left[\operatorname{IrCl}_{3}(\mathbf{9 S 3})\right]$ (9S3 = 1,4,7-trithiacyclononane) was synthesized by placing a solution of $\left[\mathrm{IrCl}_{3}\right] \cdot\left(\mathrm{H}_{2} \mathrm{O}\right)_{n}$ with $9 \mathrm{~S} 3$ in $\mathrm{MeOH}$ under microwave irradiation $(500-600 \mathrm{~W})$ for $16 \mathrm{~s}$. The desired product was isolated in $98 \%$ yield.

Syntheses of iridium polypyridine complexes have been described as being very long, implying laborious purifications while providing the complexes in very low yield. To improve the method, a microwave-assisted synthesis of photosensitive iridium(III) polypyridine complexes 424-425 was developed (Scheme 123, pathway A). ${ }^{248}$ Typically, iridium precursor and $2,2^{\prime}$-bipyridine ligand (in a $1: 3$ ratio) in ethylene glycol were

Scheme 123. Microwave-Assisted Synthesis of Iridium Polypyridine Complexes

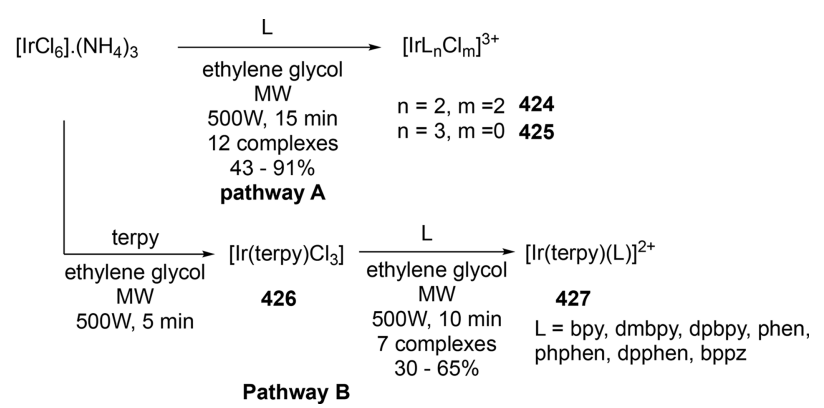


heated for $15 \mathrm{~min}$ at reflux using a microwave oven (RR-12AF, $500 \mathrm{~W}$, Mitsubisi Denki Co. Ltd., $2450 \mathrm{MHz}$ ). A saturated solution of $\mathrm{KPF}_{6}$ was added at the end to precipitate the desired product. Twelve iridium complexes with the general formula $\left[\operatorname{Ir}(\mathrm{L})_{2} \mathrm{Cl}_{2}\right]\left[\mathrm{PF}_{\mathbf{6}}\right](\mathbf{4 2 4})^{249}$ or $\left[\operatorname{Ir}(\mathrm{L})_{3}\right]\left[\mathrm{PF}_{6}\right]_{3}$ $(426)^{250}$ were recovered in $45-91 \%$ yield with different ligands (bipy, dmbpy $=4,4^{\prime}$-dimethyl-2,2' -bipyridine, dpbpy $=$ 4,4'-diphenyl-2,2'-bipyridine 260, phen, dpphen = 4,7diphenyl-1,10-phenanthroline, and bqn $=2,2^{\prime}$-biquinoline). Complexes exhibited an intense luminescence around 505$630 \mathrm{~nm}$ with various colors in acetonitrile which corresponded to a triplet $\pi-\pi^{*}$ emission. The same procedure was then applied to the synthesis of mixed-ligand Ir(III) complexes with both terpyridine and various bipyridine derivatives $[\operatorname{Ir}($ terpy)(L) $\mathrm{Cl}]^{2+} 427$ ( $\mathrm{L}=$ bpy, dmbpy, dpbpy, phen, phphen = 5phenyl-1,10-phenanthroline, dpphen, bppz = 2,3-bis (2pyridyl)pyrazine, and $\mathrm{Cl}_{2}$ ) (Scheme 123 , pathway $\mathrm{B}$ ). ${ }^{251}$ Complexes were prepared by a sequential procedure with a ligand replacement. An iridium source and terpyridine ligand (1:1 ratio) in ethylene glycol solution were refluxed in a microwave oven for $5 \mathrm{~min}$. Subsequently, the bipyridine ligand was added, and the resulting mixture was refluxed for an additional $10 \mathrm{~min}$. After addition of $\mathrm{KPF}_{6}$, the complexes were recovered by filtration with yields ranging from 30 to $65 \%$. Similarly, mixed-ligand complexes $[\operatorname{IrCl}(\mathbf{L})($ tterpy $)]\left(\mathbf{P F}_{6}\right)_{2}(\mathrm{~L}$ $=$ phen, dpphen, dmbpy, bpy, and tterpy $=4^{\prime}$-(4-tolyl)$2,2^{\prime}: 6^{\prime}, 2^{\prime \prime}$-terpyridine) were synthesized via microwave irradiation for $15 \mathrm{~min}$ and isolated in $18-63 \%$ yields. ${ }^{252}$

Microwave irradiation was also used to produce two facial tris-ortho-metalated iridium(III) complexes. Thus, fac-[Ir$\left.(\text { ppy })_{3}\right] 429$ and $f a c-\left[\operatorname{Ir}(\text { tpy })_{3}\right] 430$ (tpyH $=2$ - $(p$-tolyl $)-$ pyridine) were synthesized by reaction between $\left[\mathrm{IrCl}_{3}\right]$. $\left(\mathrm{H}_{2} \mathrm{O}\right)_{3}$ and a large excess of 2-arylpyridine 428 under microwave irradiation for $1-3 \mathrm{~min}$ (Scheme 124). ${ }^{253}$ Under

Scheme 124. Selective Microwave-Assisted Synthesis of facTris-ortho-Metalated Iridium(III) Complexes

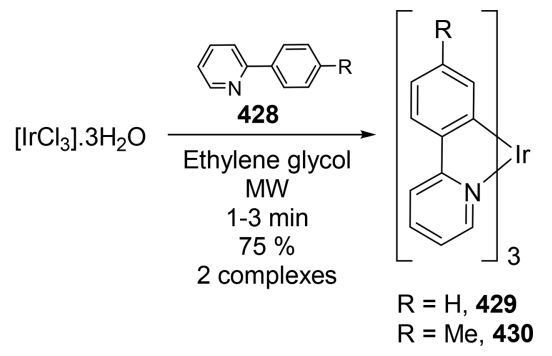

standard conditions, the reaction between iridium chloride and 428 at reflux formed the undesired dichloro-bridged dimer $\left[\mathrm{Ir}_{2} \mathrm{Cl}_{2}(\mathrm{Rpy})_{4}\right](\mathrm{R}=2$-phenyl or 2 - $(p$-tolyl $))$ as the major product. To avoid this, conditions were developed using a dehalogenating agent under reflux for several hours. Interestingly, microwave-assisted synthesis did not require the use of a dehalogenating agent, and products could be obtained quickly, efficiently, and selectively. Notably, the undesired complex could be obtained by decreasing the ligand excess while increasing the reaction time. The optimized procedure was applied to the synthesis of $\left[\operatorname{Ir}(\mathbf{p h q})_{3}\right](\mathrm{phq}=2$-phenyl-1quinoline), which was difficult to synthesize using the conventional method (after $10 \mathrm{~h}$, only trace amounts of product were detected). ${ }^{254}$ Using microwave irradiation, only 20 min was required to obtain the complex in low yield (10\%).
An electrophosphorescent device with $\left[\operatorname{Ir}(\mathbf{p h q})_{3}\right]$ as a dopant in the emitting layer was developed making use of the highly efficient orange emission of this iridium complex.

\subsection{Photochemical Synthesis of Iridium Complexes}

Light irradiation of a solution of $\left[\operatorname{IrCp}^{*}(\mathrm{CO})_{2}\right] 431$ in 2,2dimethylpropane (neopentane) at room temperature for $5 \mathrm{~h}$ led to the formation of the new hydridoneopentyliridium complex $432 .^{255}$ Due to the high instability of the resulting iridium product, the yield was estimated by reacting the complex with $\mathrm{CCl}_{4}$ until complete formation of the stable $\left[\operatorname{IrClCp} *(\mathrm{CO})\left(\mathrm{CH}_{2} \mathrm{CMe}_{3}\right)\right] 433$ (Scheme 125). Similarly,

Scheme 125. Photochemical Generation of an Iridium Complex

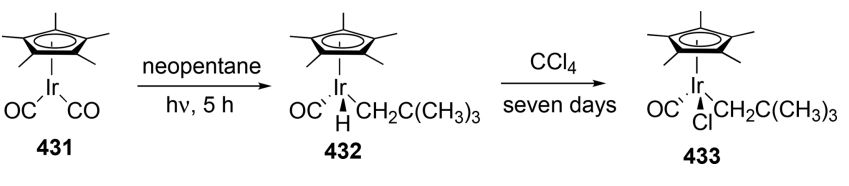

irradiation of 431 in cyclohexane, in cyclohexane- $d_{12}$, or in benzene produced cyclohexylhydrido derivatives $[\operatorname{Ir}(\mathbf{H})(\mathbf{C O})$ (L) $]\left(\mathrm{L}=\mathrm{C}_{6} \mathrm{H}_{11}, \mathrm{C}_{6} \mathrm{D}_{11}\right.$, or $\left.\mathrm{C}_{6} \mathrm{H}_{5}\right)$, which could easily be converted to the chloride derivatives $[\operatorname{IrCl}(\mathrm{CO})(\mathrm{L})]$ using $\mathrm{CCl}_{4}$.

There are few examples of diiridium(II) complexes containing no bridging ligands across the Ir-Ir bond in the literature. In this context, Mak et al. described the synthesis of $\left[\operatorname{Ir}\left(\mathrm{Cp}^{*}\right)(\boldsymbol{\mu} \text {-CO })\left(\mathrm{C}_{6} \mathrm{~F}_{4} \mathrm{CN}\right)\right]_{2}$ complexes 437 under visiblelight irradiation. A solution of $\left[\operatorname{IrCp} *(C O)_{2}\right] 431$ in $\mathrm{C}_{6} \mathrm{~F}_{5} \mathrm{CN}$ was irradiated with a tungsten lamp for $4 \mathrm{~h}$ leading to the formation of complexes 436 and 437 (Scheme 126, pathway A). ${ }^{256}$ The minor isomer 437 represented one of the few examples of such a species that did not contain a bridging ligand across the Ir-Ir bond. Notably, when placed under visible-light irradiation, the latter was transformed into the major isomer 436 which was obtained in $90 \%$ yield. Interestingly, in the presence of moisture, complex 435

Scheme 126. Photochemistry Reaction of $\left[\operatorname{IrCp} *(\mathrm{CO})_{2}\right]$ with $\mathrm{C}_{6} \mathrm{~F}_{5} \mathrm{CN}$

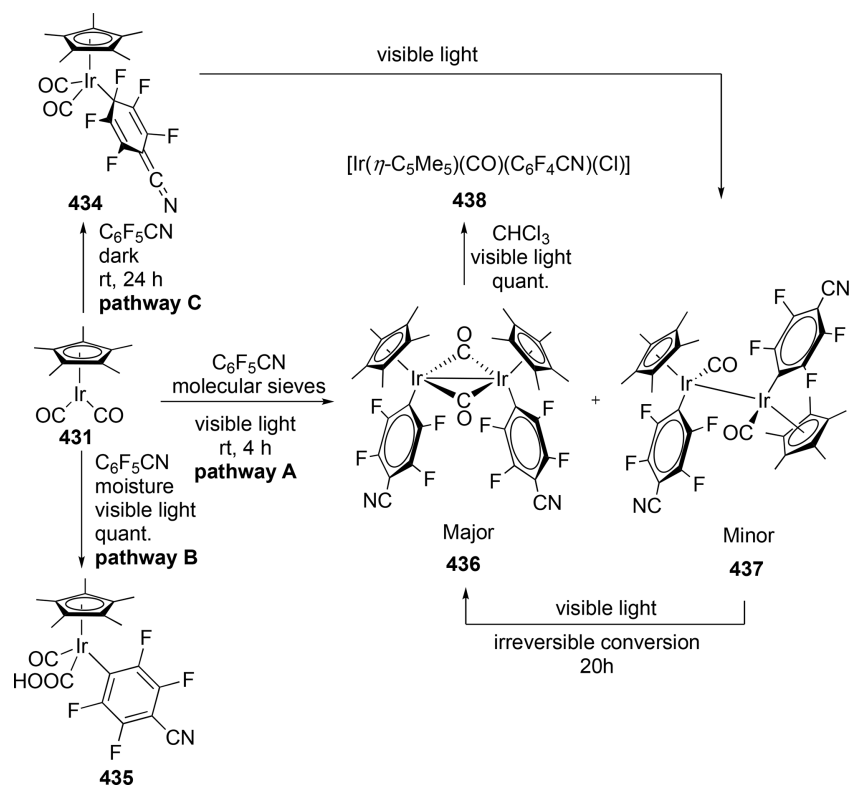


holding a formate ligand was obtained quantitatively (Scheme 126 , pathway $\mathrm{B})$. Under the same conditions, $\mathrm{C}_{6} \mathrm{~F}_{6}$ reacted under a UV lamp; however, no reaction was observed under visible-light irradiation. Additionally, in the presence of water, no analogous reaction corresponding to pathway $B$ was detected. Mechanistic investigations of reaction A were achieved by performing the reaction in the dark (Scheme 126 , pathway $\mathrm{C}$ ). These conditions resulted in $90 \%$ conversion to the iridium intermediate 434 after $24 \mathrm{~h}$ of stirring, which was then transformed into 437 under visible-light irradiation. Photochemically, $\left[\operatorname{Ir}\left(\mathrm{Cp}^{*}\right)(\boldsymbol{\mu} \text {-CO })\left(\mathrm{C}_{6} \mathrm{~F}_{4} \mathrm{CN}\right)\right]_{2} 436$ could be quantitatively transformed into $\left[\operatorname{Ir}\left(\mathbf{C p}^{*}\right)(\mathbf{C O})\left(\mathrm{C}_{6} \mathrm{~F}_{4} \mathrm{CN}\right) \mathrm{Cl}\right]$ complex 438 with no observation of the $\left[\operatorname{Ir}\left(\mathbf{C p}^{*}\right)(\mathbf{C O})\right.$ $\left.\left(\mathrm{C}_{6} \mathrm{~F}_{4} \mathrm{CN}\right)\left(\mathrm{CHCl}_{2}\right)\right]$ complex. The reaction failed in the absence of visible-light irradiation.

\section{GROUP 10}

Group 10 metal complexes are extensively studied for their catalytic properties as well as applications as bio-organometallic drugs. Palladium complexes are widely used in the laboratory or industrial scale; for example, in $\mathrm{C}-\mathrm{C}$ coupling reactions, they exhibit high catalytic properties. The introduction of different types of ligands, for example, NHC (N-heterocyclic carbene), enabled the optimization of the performances of corresponding palladium complexes. ${ }^{257}$ Since the discovery of cisplatin as an anticancer agent, platinum complexes have also been considerably studied due to their therapeutic effect. ${ }^{6,7,142}$ Similarly, nickel complexes have also been investigated for their biological properties. ${ }^{6}$

\subsection{Mechanochemical Approach}

Cyclopalladated complexes have been significantly studied not only for their wide application in organic synthesis and catalysis but also for their mesogenic, bioactive, and photoluminescent properties. Solid-state reactions of dicyclopalladated azobenzenes 439 and triphenylphosphine led to the formation of the cis-isomer-bridged complexes 440 (Scheme 127). ${ }^{258}$ It was expected that DMF present in the substrate as

Scheme 127. Mechanosynthesis of Azobenzene/

Triphenylphosphine Palladacycles
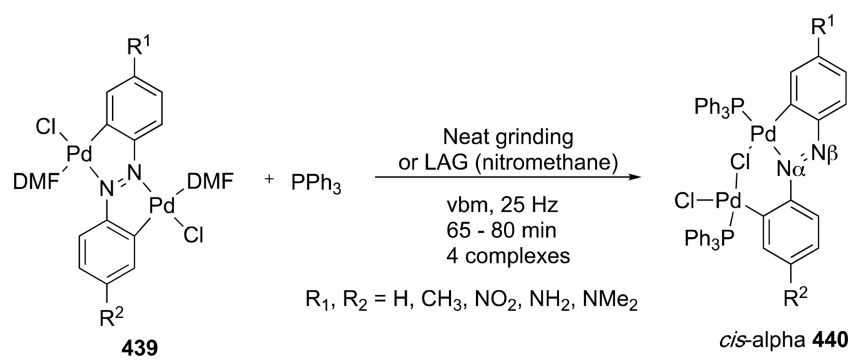

ligand could be simply displaced by the $\mathrm{PPh}_{3}$, whereas azobenzene would act as a double chelating C,N-donor. Surprisingly, in the aforementioned case, a structural rearrangement led to the formation of a bridged dipalladium complex in which azobenzene acted as a monodentate $\mathrm{C}$-donor and as a chelating C,N-donor. Notably, reactions could be performed under neat grinding, but addition of a small quantity of nitromethane shortened the reaction times.

To obtain similar cyclopalladated complexes, the Curić group reported the first mechanochemical transition-metalmediated activation of a strong phenyl $\mathrm{C}-\mathrm{H}$ bond in 2014 (Scheme 128). ${ }^{259}$ Reaction of azobenzene 441 with palladium acetate under mechanochemical grinding for $4.5 \mathrm{~h}$ led to the formation of the corresponding palladated complex 442 in $78 \%$ yield. Interestingly, in situ monitoring using solid-state Raman spectroscopy enabled both the characterization of reaction species and the direct optimization of the milling process. In this case, the first palladation, higher yielding, proved to be regioselective and much faster than the analogous reaction in solution (3 days, 39\%) (Scheme 128, step A). Interestingly, a second $\mathrm{C}-\mathrm{H}$ activation could be performed by addition of palladium acetate and grinding for $7.5 \mathrm{~h}$, yielding tetrapalladated species 443 ( $85 \%$, Scheme 128, step B). It is worth noting that the conventional solution procedures did not permit the formation of this complex to be observed.

Grinding the bisthiocarbamate ligand 444 with $\left[\mathrm{PdCl}_{2}(\mathbf{N C P h})_{2}\right]$ for $2 \mathrm{~min}$ in a vibratory ball mill led to the synthesis of the $\mathrm{Pd}^{\mathrm{II}}$-pincer complex 445 in $95 \%$ yield, through a $\mathrm{C}-\mathrm{H}$ bond activation (Scheme 129). ${ }^{260}$ Initially, the

Scheme 129. Mechanochemical Synthesis of $\mathbf{P d}^{\mathrm{II}}-$ Pincer Complex

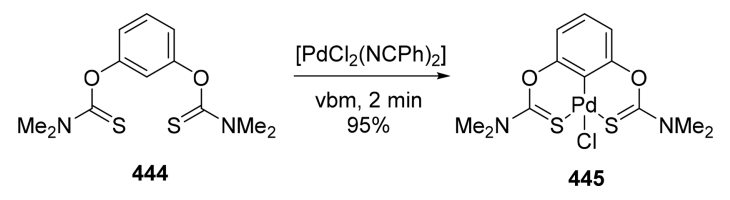

authors described the synthesis using a mortar and pestle, but a vibratory ball mill was required to perform the synthesis at the gram scale. Interestingly, formation of $\mathbf{4 4 5}$ could be observed by the formation of a paste resulting from benzonitrile release during the reaction.

In 2018, Kumar et al. described the synthesis of dimeric cyclobutenylpalladium chloride complexes 446-447 by mixing palladium(II) chloride and diphenylacetylene in a 1:2 ratio in the presence of an alcohol (Scheme 130). ${ }^{261}$ The same conditions, using only a catalytic amount of $\mathrm{PdCl}_{2}$, allowed the trimerization of diphenylacetylene into hexaphenylbenzene.

Scheme 128. Mechanosynthesis of Cyclopalladated Complexes
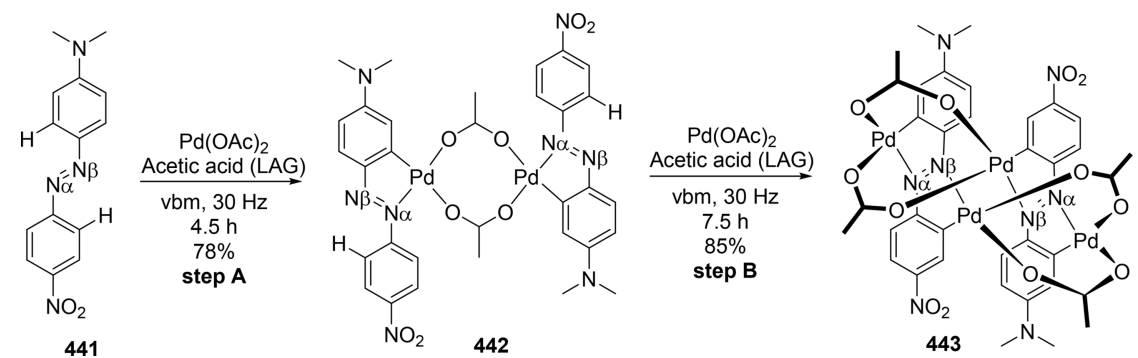
Scheme 130. Mechanosynthesis of Dimeric Cyclobutenylpalladium Chloride Complexes

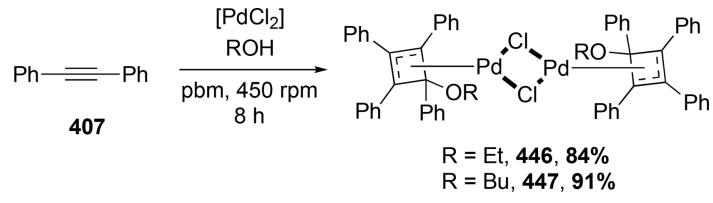

An original procedure for the synthesis of $N, N$-diaryl NHCpalladium complexes using a vibratory ball-mill Retsch MM200 was described in 2017. ${ }^{262}$ Starting from NHC-silver complexes 449, an allylpalladium(II) chloride dimer was added into a stainless steel jar and led to the formation of corresponding palladium complex $\mathbf{4 5 0}$ in $98 \%$ yield after $1.5 \mathrm{~h}$ of reaction (Scheme 131, reaction A). Starting directly from the imidazolium salt 448, a one-pot, two-step synthesis could be perfomed in the ball mill, involving metalation of the imidazolium followed by the transmetalation of the silver complex intermediate. Using this procedure, complex 450 was recovered after $1.5 \mathrm{~h}$ in $92 \%$ yield (Scheme 131, reaction B). In the same year, Adams et al. demonstrated the direct mechanosynthesis of $\left[\mathrm{Pd}(\mathrm{NHC})_{2} \mathrm{Cl}_{2}\right] \mathbf{4 5 2}$ using a mortar and pestle (Scheme 131, reaction $\mathrm{C}$ ). ${ }^{263}$ The two steps, intermediate formation of $[\mathbf{N H C}]_{2}\left[\mathbf{P d C l}_{4}\right]$ and further grinding with $\mathrm{KOH}$ to isolate 452 , were performed as a onepot reaction.

In 2015, the Orpen group demonstrated that PEPPSI-type complexes (PEPPSI $=$ pyridine-enhanced precatalyst preparation stabilization and initiation), which are well-known for their catalytic properties, could be mechanosynthesized following a two-step procedure. ${ }^{264}$ Using an agate mortar and pestle, grinding of an imidazolium chloride $(448,451$, and 453), pyridinium chloride, and the metallic salt $\mathrm{K}_{2} \mathrm{MCl}_{4}(\mathrm{M}=$ $\mathrm{Pd}, \mathrm{Pt}$ ) produced intermediate salt 454, which upon grinding with $\mathrm{KOH}$ produced the desired PEPPSI complexes 455 in quantitative yield (Scheme 132). Syntheses involving platinum salts required more time than with palladium salts. The first step could be followed using PXRD, but attempts to crystallize the intermediate salt resulted in the spontaneous deprotonation of the pyridinium counterions to form $\left[\mathrm{MCl}_{3}(\mathbf{P y})\right]^{-}[\mathbf{N H C H}]^{+}$. In comparison with a solvent-based strategy, PEPPSI complexes are formed by refluxing a mixture of $\mathrm{PdCl}_{2}, \mathrm{~K}_{2} \mathrm{CO}_{3}$, and the imidazolium salt in neat 3- chloropyridine for $16 \mathrm{~h}$, resulting in the products in excellent yields, but the excess of pyridine had to be distilled at the end of the procedure.

The fast coordination of imidazole (Him) 458 and pyrazole $(\mathrm{Hpz}) 459$ on platinum and palladium in the solid state was described by the same group (Scheme 133). ${ }^{265}$ Various strategies were investigated for the synthesis of discrete complexes with the general formula $\left[\mathrm{M}(\mathrm{L})_{2} \mathrm{Cl}_{2}\right] 456(\mathrm{M}=$ $\mathrm{Pd}, \mathrm{Pt} ; \mathrm{L}=\mathrm{Him}, \mathrm{Hpz})$, by grinding ligands with either $\mathrm{K}_{2} \mathrm{MCl}_{4}$ (Scheme 133, reaction, A) or with $\mathrm{MCl}_{2}$ or $\mathrm{M}(\mathrm{OAc})_{2}$ using a mortar and pestle (Scheme 133, reaction B). Using $\mathrm{MCl}_{2}$ and $\mathrm{M}(\mathrm{OAc})_{2}$ has the main advantage that they produce the desired complex without salts. Conversely, when $\mathrm{K}_{2} \mathrm{MCl}_{4}$ was used (reactions $\mathrm{A}$ and $\mathrm{E}$ ), $\mathrm{KCl}$ was formed as a byproduct but could be easily removed by washing the reaction mixture with water. Cis- $\left[\mathbf{M C l}_{\mathbf{2}}(\mathbf{H i m})_{2}\right](\mathrm{M}=\mathrm{Pd}, \mathbf{P t})$, trans- $\left[\mathbf{P d C l}_{\mathbf{2}}(\mathbf{H p z})_{2}\right]$, and cis- $\left[\mathbf{P t C l}_{2}(\mathbf{H p z})_{2}\right]$ were the only isomers recovered after mechanochemical treatment. Interestingly, cis- $\left[\mathbf{P d C l}_{2}(\mathbf{H i m})_{2}\right]$ could easily be transformed into the trans-isomer at high temperatures. Using a different strategy, salts with the general formula $[\mathrm{HL}]_{2}\left[\mathbf{M C l}_{4}\right]$ were synthesized using mechanochemistry with 458 and 459 as their hydrochloride salts (Scheme 133 , reactions $\mathrm{D}$ and $\mathrm{E}$ ). They were easily transformed into the desired discrete complexes when ground with a base (Scheme 133 , reaction $\mathrm{C}$ ). $\mathrm{Ag}_{2} \mathrm{O}$ was preferred to $\mathrm{KOH}$ or $\mathrm{K}_{2} \mathrm{CO}_{3}$ because it avoided the formation of $\mathrm{KCl}$ and the subsequent formation of $\mathrm{K}_{2} \mathrm{MCl}_{4}$. Notably, $\left[\mathrm{PdCl}_{2}(\mathrm{Him})_{2}\right]$ could not be synthesized by grinding $\left[\mathbf{H}_{2} \mathbf{I m}\right]_{2}\left[\mathbf{P d C l}_{4}\right]$ with a base; the reaction produced only a mixture of the unprecedented $\left[\mathbf{P d}(\mathbf{H i m})_{4}\right] \mathbf{C l}_{2}$ complex and $\mathrm{PdCl}_{2}$. Similarly, $\left[\mathbf{P d}(\mathbf{H p z})_{4}\right] \mathbf{C l}_{2}$ was recovered after mechanochemical treatment of $\mathrm{PdCl}_{2}$ with Hpz. In order to efficiently synthesize $\left[\mathbf{P d}\left(\mathrm{Hpz}_{2} \mathbf{C l}_{2}\right]\right.$, the palladium source was changed from $\mathrm{PdCl}_{2}$ or $\mathrm{K}_{2} \mathrm{PdCl}_{4}$ to $\mathrm{Pd}(\mathrm{OAc})_{2}$. It is worth noting that the reaction of $\mathrm{K}_{2} \mathrm{PtCl}_{4}$ and imidazole did not occur in solution but only in a ball mill to yield $\left[\mathrm{PtCl}_{2}(\mathrm{Him})_{2}\right]$.

In 2018, the Frišcic group developed a straightforward mechanochemical procedure for the oxidation of elementary palladium into soluble $\left[\mathrm{M}_{2} \mathbf{P d X} \mathbf{X}_{4}\right]$ salts $460\left(\mathrm{M}=\mathrm{K}, \mathrm{NH}_{4}\right)$ (Scheme 134). ${ }^{266}$ Subsequently, these compounds could be milled with a variety of ligands (dppf, triphenylphosphine, tricyclohexylphosphine and tris(pentafluorophenyl)phosphine $\left(\mathrm{ppfp}_{3}\right), \mathrm{ACN}$, and acac) to form the corresponding coordination complexes 461 and 462. Direct synthesis of

Scheme 131. Mechanosynthesis of NHC-palladium Complexes

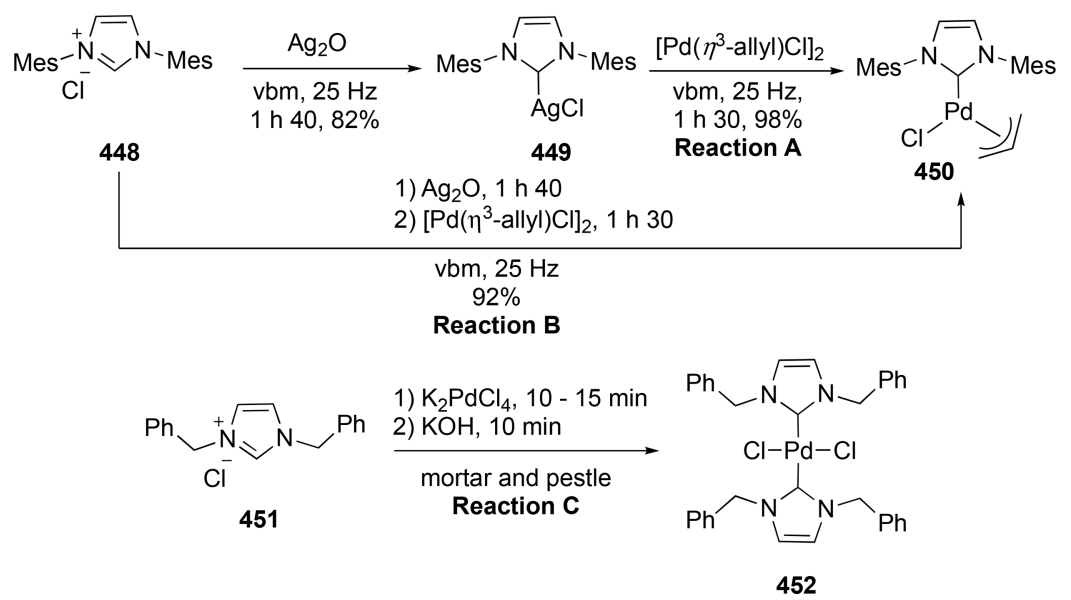


Scheme 132. Mechanosynthesis of PEPPSI-Type Complexes

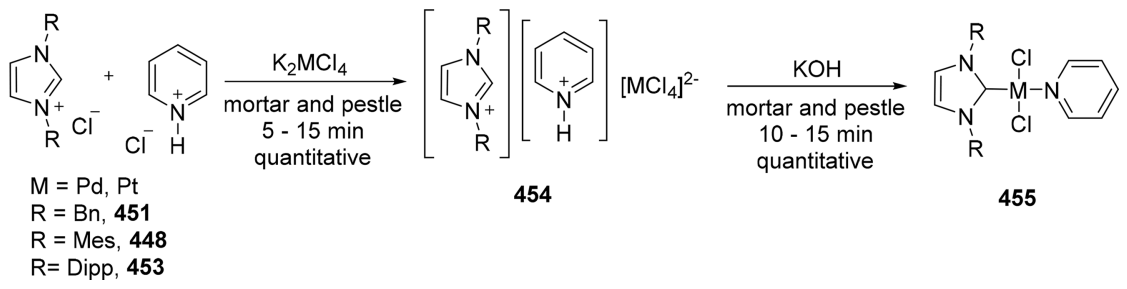

Scheme 133. Coordination of Platinum and Palladium with Pyrazole and Imidazole

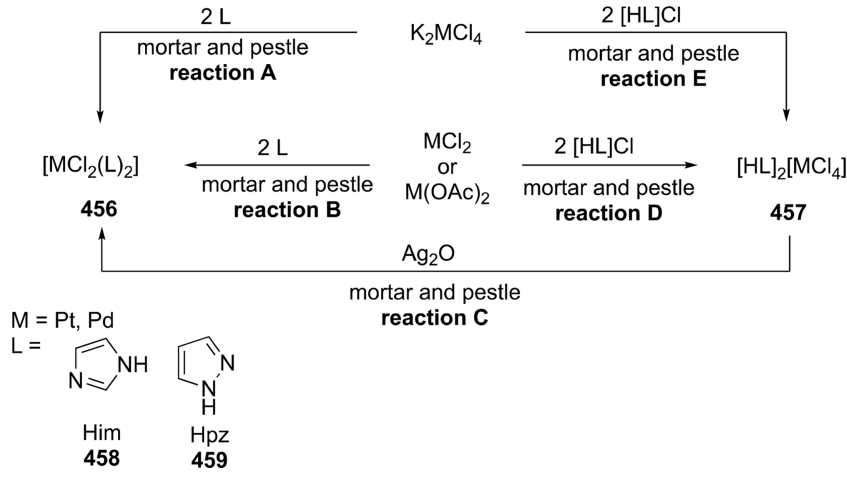

Scheme 134. Mechanochemical Oxidation of Pd Metal

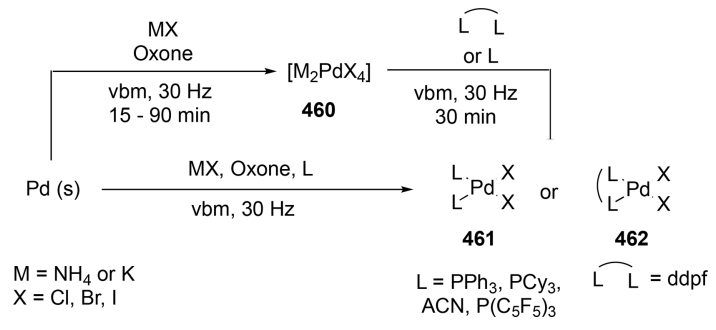

$\left[\mathrm{PdL}_{2} \mathrm{X}_{2}\right] 461$ was also described starting from palladium metal, the ligand, chloride salt, and the oxidant. Interestingly, this method avoids the use of aggressive acids, molten salts, or cyanides, which are usually used to perform the oxidation.

In 2002, Balema et al. described the first mechanosynthesis of platinum complexes. ${ }^{267}$ Following standard procedures, cisbis(triphenylphosphine)platinum(II) dichloride 463 is formed by reacting $\mathrm{PtCl}_{2}$ with $\mathrm{PPh}_{3}$ under melting conditions (292 ${ }^{\circ} \mathrm{C}$ ), however, with the concomitant formation of around $20 \%$ of the trans-isomer. Interestingly, ball milling (Spex-8000 mill) of $\mathrm{PtCl}_{2}$ with 2 equiv of $\mathrm{PPh}_{3}$ for $1 \mathrm{~h}$ at room temperature selectively produced cis- $\left[\mathbf{P t}\left(\mathbf{P P h}_{3}\right)_{2}\right] \mathbf{C l}_{2} \quad 463$ (98\% yield) without any side products (such as triphenylphosphine oxide or other phosphorus-containing species), as shown in solidstate ${ }^{31} \mathrm{P}$ MAS (magic-angle spinning) NMR analysis (Scheme 135, step A). The corresponding carbonate adduct 464 could be synthesized by milling with $\mathrm{K}_{2} \mathrm{CO}_{3}$ (70\% yield), while $\mathrm{Ag}_{2} \mathrm{CO}_{3}$ was the only metal carbonate effective for this transformation in solution. Despite this, around 20\% of triphenylphosphine oxide was detected in the milling process (Scheme 135, step B). The direct preparation of 464 was attempted from $\mathrm{PtCl}_{2}, \mathrm{PPh}_{3}$, and $\mathrm{K}_{2} \mathrm{CO}_{3}$. The desired complex could be detected; however, consumption of intermediate 463 remained incomplete after $14 \mathrm{~h}$.

In 2013, the mechanosynthesis of $\left[\mathrm{Ni}(\mathrm{OAc})_{2}(\mathrm{TMP})_{2}\right] 466$ (TMP = trimethoprim; (5-(3,4,5-trimethoxybenzyl)pyrimidine-2,4-diamine)) was described, with the purpose of developing a coordination complex derived from an active pharmaceutical ingredient (Scheme 136). ${ }^{268}$ Indeed, trimetho-

Scheme 136. Mechanosynthesis of $\left[\mathrm{Ni}(\mathrm{TMP})_{2}(\mathrm{OAc})_{2}\right]$

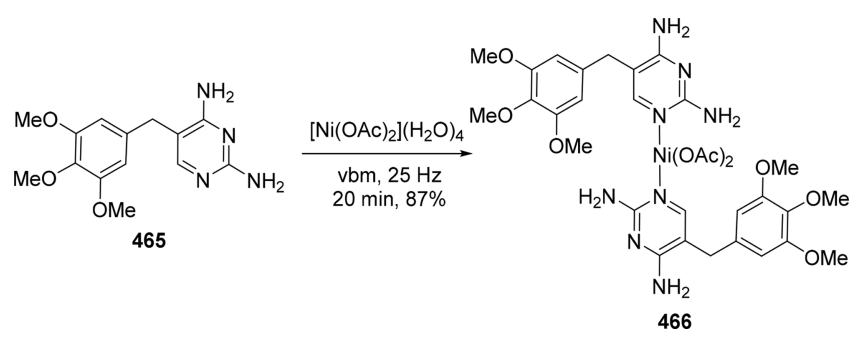

prim 465 is a bacteriostatic antibiotic used to treat urinary infections. TMP 465 and $\left[\mathrm{Ni}(\mathrm{OAc})_{2}\right] \cdot 4 \mathrm{H}_{2} \mathrm{O}$ were ground in equimolar amounts for $20 \mathrm{~min}$ in a vibratory ball mill operated at $25 \mathrm{~Hz}$ in a stainless steel jar, forming complex 466 in $87 \%$ yield. Mechanosynthesis was faster than solution-based conventional synthesis, which required $2 \mathrm{~h}$ in methanol at reflux and five more days at room temperature for recrystallization.

Nickel(II) coordination compounds with diimine ligands are generally known as Brookhart-type catalysts. Several procedures have been developed over time to synthesize this type of complex. Following a standard method, metalation is performed with $\left[\mathrm{NiBr}_{2}(\mathrm{DME})\right]$ (DME = 1,2-dimethoxyethane) in solution during $24 \mathrm{~h}$. The Duarte group has developed an efficient mechanochemical alternative using vibratory ball milling (Retsch MM400) (Scheme 137). ${ }^{239}$ Using $\left[\mathrm{NiBr}_{2}(\mathrm{DME})\right]$ as the metal source resulted in corresponding complex 467 in quantitative yield after only 20 min of milling. Interestingly, using anhydrous $\mathrm{NiBr}_{2}$ instead of $\left[\mathrm{NiBr}_{2}(\mathrm{DME})\right]$, the same product was obtained in quantitative yield after $30 \mathrm{~min}$ of grinding. The use of anhydrous $\mathrm{NiBr}_{2}$ in solution was already described using glacial acetic acid as solvent, but this approach was found less efficient $(67 \%$ yield $)$. Finally, preparation of $\left[\mathbf{N i}(\boldsymbol{\eta} \text {-Ind })_{2}\right]$ (Ind $=$

Scheme 135. First Mechanosynthesis of Platinum Complexes

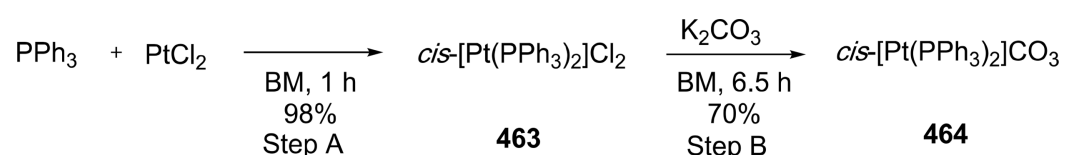


Scheme 137. Mechanochemical Synthesis of BrookhartType Nickel Complexes

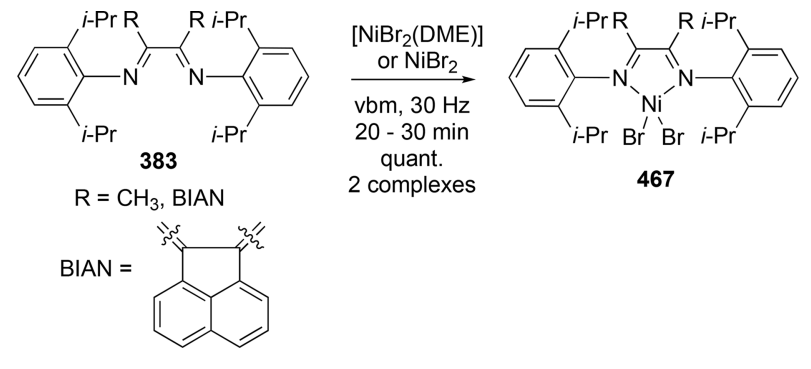

indene) was attempted, but the resulting red solid decomposed before any characterization could be performed, due to the supposed instability toward oxygen and moisture.

Thiosemicarbazone metal complexes have been extensively studied due to their interesting donor properties, their structural diversity, and their biological and catalytic properties. In 2012, three different techniques (ball mill, electrochemistry, and solution conditions) were used to form $\mathrm{Ni}$ (II) salicylaldehyde 4-methyl and 4-phenylthiosemicarbazonato complexes 469 and 470. ${ }^{269}$ Three different geometries could be obtained for the final complexes, either tetrahedral, square planar, or octahedral. In solution, the 4-methyl-substituted complex was recovered with a tetrahedral structure, while the 4-phenyl was obtained with a square planar structure. Additionally, products were obtained in low yields (respectively, 41 and $85 \%$ ) after $30 \mathrm{~min}$ of reaction. Electrochemical synthesis using a nickel anode in methanol for 1 day furnished the octahedral complex 469 with the 4-methyl derivative and the square planar one with the 4-phenyl counterpart, in 78 and $86 \%$ yield, respectively. When the reaction is performed using mechanochemistry, only the octahedral nickel complexes 469 and 470 were recovered quickly and quantitatively (Scheme 138). This study clearly demonstrates that the activation technique can affect the outcome of the reaction.

Scheme 138. Mechanosynthesis of Ni(II) Salicylaldehyde Thiosemicarbazonato Complexes
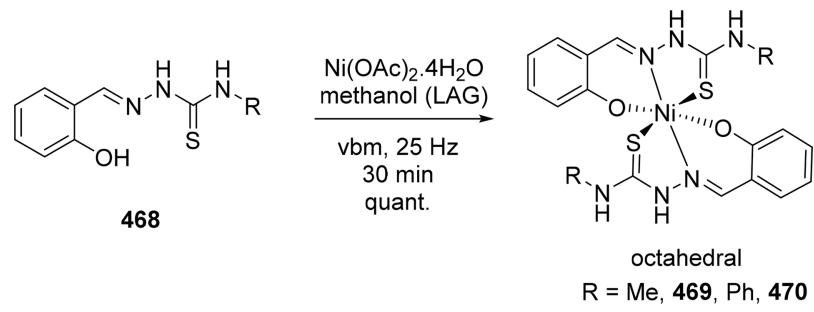

In 2014, the James group reported the mechanochemical synthesis of Salen-nickel complexes through the formation of a diimine and further reaction with nickel(II) acetate (Scheme 139). ${ }^{270}$ The final nickel complex 473 could be obtained in its pure form in $1 \mathrm{~h}$, however, as a different polymorph than classically obtained. Subsequently, the same group developed the scale-up of this reaction using twin-screw extrusion. ${ }^{271}$ The application of different activation techniques resulted in the isolation of a dimeric form of the expected complex (474).

As for ferrocene, nickelocene $\mathbf{4 7 5}$ could be synthesized by grinding $\mathrm{NiCl}_{2}$ and $\mathrm{TlCp}$ in a 1:1.8 ratio for $60 \mathrm{~min}$ using an original homemade vibratory ball mill (90\% yield, Scheme 140). ${ }^{146}$ Similarly, grinding together $\mathrm{NiCl}_{2} \cdot 6 \mathrm{H}_{2} \mathrm{O}$ with para- toluidine in a $1: 2$ stoichiometric ratio for 15 min provided dichlorobis(paratoluidine) nickel(II) in $95 \%$ yield. ${ }^{228}$ Using an analogous strategy, and as reported for cobalt complexes, $\left[\mathrm{NiCl}_{2}(4-\mathrm{PhPy})_{4}\right]$ could be mechanosynthesized directly and easily from 4-PhPy and $\mathrm{NiCl}_{2}$ and confirmed by PXRD analysis. $^{226}$

\subsection{Microwave Irradiation}

Between 2010 and 2016, the Jain group reported the microwave-assisted synthesis of numerous nickel(II) complexes with Schiff bases as ligands (Table 7, see Figure 1 for the structures of ligands). Syntheses were performed in an openglass vessel using a modified microwave open model 2001 ETB with a rotating tray. Microwave reactions were performed using on/off cycles to control the temperature. $\left[\mathrm{Ni}(84)_{2}\right] \cdot 2 \mathbf{H}_{2} \mathbf{O},{ }^{97}$ $\left[\mathrm{Ni}(\mathbf{8 3})\left(\mathbf{H}_{2} \mathbf{O}\right)_{3}\right] \mathbf{C l}^{97}[\mathbf{N i}(\mathbf{8 8})] \cdot 2 \mathbf{H}_{2} \mathbf{O}^{98}\left[\mathbf{N i}(\mathbf{8 1})_{2}\left(\mathbf{H}_{2} \mathbf{O}\right)_{2}\right]_{,}{ }^{99}$ $\left[\mathbf{N i}(\mathbf{8 2})_{2}\left(\mathbf{H}_{\mathbf{2}} \mathbf{O}\right)_{2}\right],{ }^{99}[\mathbf{N i}(\mathbf{8 5})] \cdot 3 \mathbf{H}_{\mathbf{2}} \mathbf{O},^{100}\left[\mathbf{N i}(\mathbf{8 6})_{2}\right] \cdot \mathbf{2} \mathbf{H}_{\mathbf{2}} \mathbf{O}{ }^{100}$ $\left[\mathrm{Ni}(74)\left(\mathrm{H}_{2} \mathrm{O}\right)_{3}\right],{ }^{95}[\mathrm{Ni}(\mathbf{7 5}) \mathbf{C l}] \cdot 3 \mathbf{H}_{2} \mathbf{O},{ }^{95}\left[\mathrm{Ni}(\mathbf{7 6})_{2} \mathrm{Cl}_{2}\right] \cdot$ $\mathbf{2} \mathbf{H}_{\mathbf{2}} \mathrm{O}{ }^{235}\left[\mathrm{Ni}(77)_{2} \mathrm{Cl}_{2}\right] \cdot \mathbf{2} \mathbf{H}_{\mathbf{2}} \mathbf{O},{ }^{235}\left[\mathrm{Ni}(\mathbf{7 8})_{2}\right] \cdot \mathbf{2} \mathbf{H}_{\mathbf{2}} \mathbf{O},{ }^{96}[\mathbf{N i}-$ $\left.(\mathbf{8 0})_{2}\left(\mathrm{H}_{2} \mathrm{O}\right)_{2}\right],{ }^{96}\left[\mathrm{Ni}(69)_{2}\left(\mathbf{H}_{2} \mathrm{O}\right)_{2}\right] \mathbf{C l}_{2},{ }^{272}$ and $\left[\mathrm{Ni}(70)_{2}\right]^{272}$ (Table 7, entries 2-16) were synthesized using a similar procedure. Before performing microwave irradiation, the appropriate ligand and $\mathrm{NiCl}_{2} \cdot 6 \mathrm{H}_{2} \mathrm{O}$ were ground in a stoichiometric ratio $(1: 1$ or $2: 1)$. After $6-8 \mathrm{~min}$ of irradiation in $3-5 \mathrm{~mL}$ of dry EtOH, complexes were obtained in high yields (81-86\%). Microwave activation outperformed classical conditions, reducing reaction times from hours to only a few minutes and increasing the yields from $60-70 \%$ to more than $80 \%$.

In the same way, the microwave-assisted synthesis of $[\mathrm{Ni}(7 \mathbf{1})] \cdot \mathrm{H}_{2} \mathbf{O}$ was described in 2014 . In only $5 \mathrm{~min}$, the complex was produced in $85 \%$ yield (Table 7 , entry 18). ${ }^{134}$ The $\left[\mathrm{Ni}(79)_{2}\right]$ complex could be obtained from the addition of nickel(II) acetate tetrahydrate to 79 in EtOH under irradiation at $600 \mathrm{~W}$ for $20 \mathrm{~s}$ (Table 7 , entry 17). ${ }^{133}$

The synthesis of macrocyclic $\mathrm{Ni}(\mathrm{II})$ complex $[\mathrm{Ni}(\mathbf{8 9})]$ under microwave irradiation was reported by Ali et al. (Table 7, entry 19). ${ }^{236}$ The reaction time was considerably reduced from hours to minutes, and the yield was increased (74-80\%) in comparison with the 55\% yield obtained under reflux. Similarly, [Ni(87)] synthesis was described in 2009 by an identical procedure using $\mathrm{H}_{2} \mathrm{~L}$ as ligand. The complex was recovered in $1 \mathrm{~min}$ and in $88 \%$ yield (Table 7 , entry 1). ${ }^{135}$ Recently, in 2017, microwave syntheses of $\left[\mathrm{Ni}(103)_{2}\right]$, $[\mathrm{Ni}(104) \mathrm{Cl}],[\mathrm{Ni}(105) \mathrm{Cl}]$, and $\left[\mathrm{Ni}(106)_{2}\right]$ were performed in a few minutes using DMF as the solvent (Table 7, entries $20-23)^{273}$

Alias et al. described the microwave synthesis of mixed phenanthroline and benzothiazole nickel complex 476 (Scheme 141), following the same procedure used for the synthesis of vanadium complex 47 (Scheme 11). After $20 \mathrm{~s}$ of microwave irradiation with a few drops of solvent, the desired $\mathrm{Ni}$ complex was obtained in $97 \%$ yield and was isostructural to the corresponding vanadium complex. ${ }^{83}$

The same methodology as for a ruthenium complex (Scheme 80) could be applied for $\left[\mathrm{Ni}_{2}(\mathbf{9} \text {-atc })_{4}\left(\mathbf{H}_{2} \mathbf{O}\right)(\mathbf{P y})_{4}\right] \cdot$ $2 \mathrm{H}_{2} \mathrm{O}$, synthesized by heating a solution of $\mathrm{NiCO}_{3} \cdot 2 \mathrm{Ni}(\mathrm{OH})_{2}$, 9-anthracenecarboxylic acid, and pyridine in an $\mathrm{EtOH} /$ water mixture via microwave irradiation. ${ }^{174}$ A two-step program was used consisting ofa $20 \mathrm{~min}$ heating ramp up to $150{ }^{\circ} \mathrm{C}$ followed by a $2 \mathrm{~h}$ isotherm at $150{ }^{\circ} \mathrm{C}$. After removal of the volatiles, the corresponding complex $\left[\mathrm{Ni}_{2}(\mathbf{9} \text {-atc })_{4}\left(\mathrm{H}_{2} \mathbf{O}\right)\right.$ $\left.(\mathrm{Py})_{4}\right] \cdot 2 \mathrm{H}_{2} \mathrm{O}$ was recovered in $56 \%$ yield. 
Scheme 139. Nickel-Salen Complexes Obtained through Ball Milling and Twin-Screw Extrusion

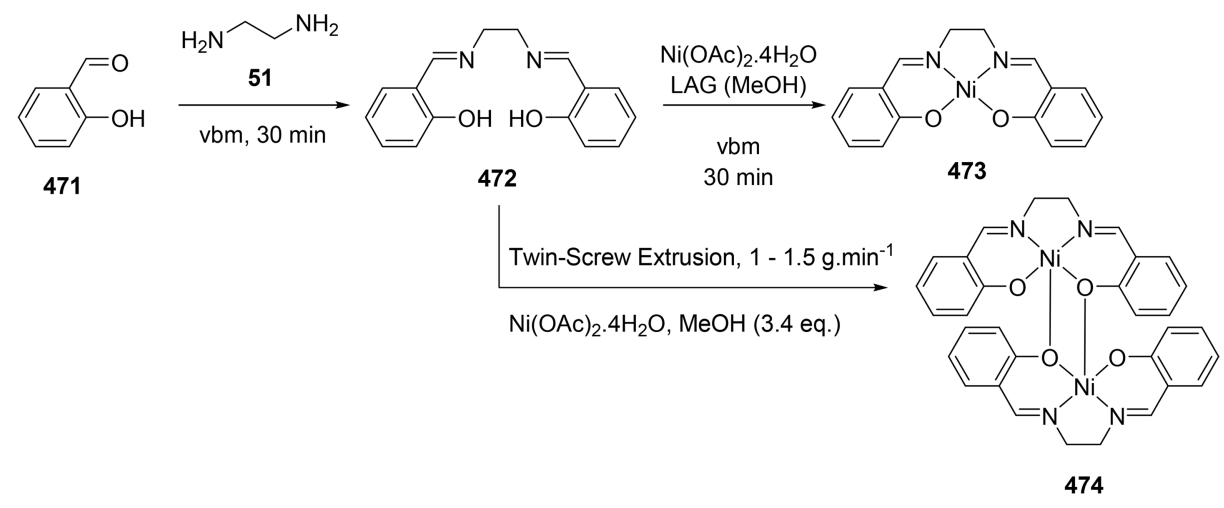

Scheme 140. Mechanosynthesis of Nickelocene

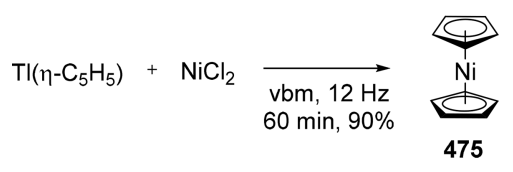

In the Group 9 section, luminol derivative 393 was used to coordinate cobalt (Scheme 112). Similarly, nickel(II) complex 477 could be prepared in less than $10 \mathrm{~min}$ and in $84 \%$ yield starting from $\mathrm{NiCl}_{2}$ (Scheme 142). Once again, the coordination of luminol derivative 393 on nickel induced a shortening of the fluorescence lifetime. ${ }^{237}$

The Zeise's salt $\left[\mathbf{K P t C l}_{3}\left(\mathbf{C}_{\mathbf{2}} \mathbf{H}_{4}\right)\right]$ was the first organometallic compound isolated in essentially pure form. The first syntheses of this complex required prolonged reaction times (7-14 days) and the use of high pressures. Since then, catalytic processes have been developed with tin chloride to yield the complex in only a few hours at atmospheric pressure but have led to difficulties in product isolation. To solve these issues, Shoemaker et al. described the microwave-assisted synthesis of $\left[\mathrm{KPtCl}_{3}\left(\mathrm{C}_{2} \mathrm{H}_{4}\right)\right]$ starting from a solution of $\mathrm{K}_{2} \mathrm{PtCl}_{4}$ and ethene ( $50 \mathrm{psi})$ in a mixture of water:EtOH:HCl. ${ }^{274}$ After only $15 \mathrm{~min}$, the product could be recovered in $89 \%$ yield after a simple filtration/evaporation workup.

Similarly, the microwave-assisted synthesis of cisplatin, the first FDA-approved $\mathrm{Pt}$ (II) drug for which anticancer activity is known worldwide, has also been described. ${ }^{275}$ Thus, cis$\left[\mathrm{Pt}\left(\mathrm{NH}_{3}\right)_{2} \mathrm{Cl}_{2}\right]$ was synthesized in more than $80-90 \%$ yield whereby an aqueous solution of $\mathrm{K}_{2} \mathrm{PtCl}_{4}, \mathrm{KCl}$, and $\mathrm{NH}_{4} \mathrm{OAc}$ in a $1: 2: 4$ ratio was heated at $100{ }^{\circ} \mathrm{C}$ under microwave irradiation for $15 \mathrm{~min}$ (Scheme 143). Notably, a simple recrystallization was sufficient to recover the pure complex. The entire procedure lasted $80 \mathrm{~min}$, thus opening the door to the

Table 7. Synthesis of Ni(II)-Schiff Base Complexes by Microwave Irradiation versus Conventional Heating ${ }^{a}$

\begin{tabular}{|c|c|c|c|c|c|c|}
\hline \multirow[b]{2}{*}{ entry } & \multirow[b]{2}{*}{ nickel complex } & \multicolumn{2}{|c|}{ microwave synthesis } & \multicolumn{2}{|c|}{ conventional heating } & \multirow[b]{2}{*}{ ref } \\
\hline & & time $(\min )$ & yield (\%) & time & yield (\%) & \\
\hline 1 & {$[\mathrm{Ni}(87)]$} & 1 & 88 & 5 & 79 & 135 \\
\hline 2 & {$\left[\mathrm{Ni}(84)_{2}\right] \cdot 2 \mathrm{H}_{2} \mathrm{O}$} & 7 & 80 & 7 & 67 & 97 \\
\hline 3 & {$\left[\mathrm{Ni}(83)\left(\mathrm{H}_{2} \mathrm{O}\right)_{3}\right] \mathrm{Cl}$} & 7 & 81 & 7 & 68 & 97 \\
\hline 4 & {$[\mathrm{Ni}(88)] \cdot 2 \mathrm{H}_{2} \mathrm{O}$} & 8 & 80 & 7 & 68 & 98 \\
\hline 5 & {$\left[\mathrm{Ni}(\mathbf{8 1})_{2}\left(\mathrm{H}_{2} \mathrm{O}\right)_{2}\right]$} & 7 & 83 & 6 & 65 & 99 \\
\hline 6 & {$\left[\mathrm{Ni}(82)_{2}\left(\mathrm{H}_{2} \mathrm{O}\right)_{2}\right]$} & 8 & 80 & 7 & 63 & 99 \\
\hline 7 & {$[\mathrm{Ni}(85)] \cdot 3 \mathrm{H}_{2} \mathrm{O}$} & 7 & 81 & 7 & 69 & 100 \\
\hline 8 & {$\left[\mathrm{Ni}(86)_{2}\right] \cdot 2 \mathrm{H}_{2} \mathrm{O}$} & 7 & 84 & 8 & 71 & 100 \\
\hline 9 & {$\left[\mathrm{Ni}(74)\left(\mathrm{H}_{2} \mathrm{O}\right)_{3}\right]$} & 8 & 84 & 8 & 66 & 95 \\
\hline 10 & {$[\mathrm{Ni}(75) \mathrm{Cl}] \cdot 3 \mathrm{H}_{2} \mathrm{O}$} & 7 & 84 & 7 & 66 & 95 \\
\hline 11 & {$\left[\mathrm{Ni}(76)_{2} \mathrm{Cl}_{2}\right] \cdot 2 \mathrm{H}_{2} \mathrm{O}$} & 7 & 84 & 7 & 69 & 235 \\
\hline 12 & {$\left[\mathrm{Ni}(77)_{2} \mathrm{Cl}_{2}\right] \cdot 2 \mathrm{H}_{2} \mathrm{O}$} & 7 & 84 & 8 & 69 & 235 \\
\hline 13 & {$\left[\mathrm{Ni}(78)_{2}\right] \cdot 2 \mathrm{H}_{2} \mathrm{O}$} & 8 & 83 & 7 & 70 & 96 \\
\hline 14 & {$\left[\mathrm{Ni}(80)_{2}\left(\mathrm{H}_{2} \mathrm{O}\right)_{2}\right]$} & 7 & 85 & 7 & 69 & 96 \\
\hline 15 & {$\left[\mathrm{Ni}(69)_{2}\left(\mathrm{H}_{2} \mathrm{O}\right)_{2}\right] \mathrm{Cl}_{2}$} & 9 & 80 & 8 & 67 & 272 \\
\hline 16 & {$\left[\mathrm{Ni}(70)_{2}\right]$} & 8 & 84 & 7 & 71 & 272 \\
\hline 17 & {$\left[\mathrm{Ni}(79)_{2}\right]$} & $20 \mathrm{~s}$ & 90 & 5 & 76 & 133 \\
\hline 18 & {$[\mathrm{Ni}(7 \mathbf{1})] \cdot \mathrm{H}_{2} \mathrm{O}$} & 5 & 85 & 8 & 61 & 134 \\
\hline 19 & {$[\mathrm{Ni}(89)]$} & $8-10$ & $74-80$ & hours & 55 & 236 \\
\hline 20 & {$\left[\mathrm{Ni}(103)_{2}\right]$} & 2 & 74 & 4.5 & 62 & 273 \\
\hline 21 & {$[\mathrm{Ni}(104) \mathrm{Cl}]$} & 1.5 & 77 & 2 & 62 & 273 \\
\hline 22 & {$[\mathrm{Ni}(105) \mathrm{Cl}]$} & 2.5 & 68 & 3 & 54 & 273 \\
\hline 23 & {$\left[\mathrm{Ni}(106)_{2}\right]$} & 2.1 & 73 & 3.5 & 65 & 273 \\
\hline
\end{tabular}

${ }^{a}$ See Figure 1 for the structure of ligands. 
Scheme 141. Microwave Synthesis of a Benzothiazole/Phenanthroline Mixed Nickel Complex

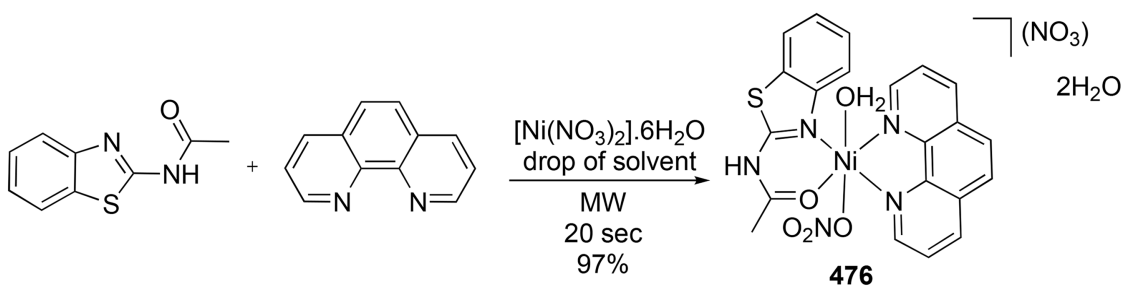

\section{Scheme 142. Microwave Synthesis of a Ni-Luminol} Complex

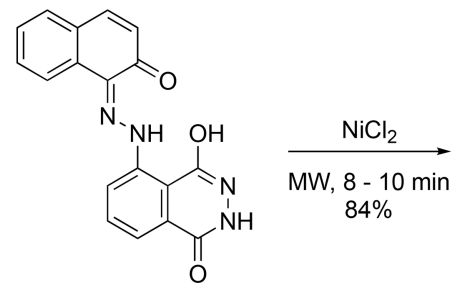

393

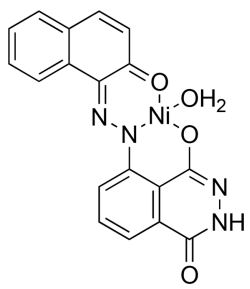

477
Scheme 143. Expedient Microwave-Assisted Formation of Cisplatin

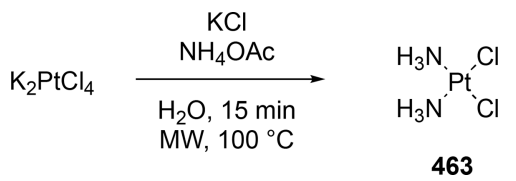

synthesis of the ${ }^{195 \mathrm{~m}} \mathrm{Pt}$ corresponding complex and the related biological studies. This result is a drastic improvement compared to classical solution conditions, which required 2$3 \mathrm{~h}$ from the beginning of the reaction to isolation of the complex. The conditions were optimized to avoid the formation of the trans-isomer and the Magnus green salt $\left(\left[\mathbf{P t}\left(\mathrm{NH}_{3}\right)_{3} \mathbf{C l}\right]\left[\mathbf{P t}\left(\mathrm{NH}_{3}\right) \mathbf{C l}_{3}\right]\right)$. Interestingly, higher temperatures led to the formation of the trans-isomer, and higher concentrations of $\mathrm{NH}_{4} \mathrm{OAc}$ salt led to the formation of the Magnus salt.

Carlsson and Eliasson described the one-pot microwave synthesis of mono- or diarylalkynyl platinum complexes 478 and 480 with phosphine or phosphite ligands. Reaction of $\mathrm{PtCl}_{2}$ with acetylide 479 and phosphorus ligand resulted in the monosubstituted complexes, whereas addition of $\mathrm{CuI}$ allowed the formation of the disubstituted complexes (Scheme 144). ${ }^{276}$ The microwave-assisted reaction was fast, and complexes were obtained in good yields. It is worth noting that this synthesis did not require prior preparation of the intermediate $\left[\mathbf{P t C l}_{2}\left(\mathbf{P R}_{3}\right)_{2}\right]$ complex, which was formed in situ during the microwave procedure.
In 2003, Januário-Charmier et al. described the $[2+3]$ cycloaddition of nitrone $\mathbf{4 8 1}$ with different platinum sources containing EtCN ligands, the anionic platinum(II) $\left[\mathbf{P P h}_{3}\left(\mathbf{C H}_{2} \mathbf{P h}\right)\right]\left[\mathbf{P t C l}_{3}(\mathbf{E t C N})\right] 482$ (Scheme 145, reaction A), the neutral platinum(II) $\left[\mathbf{P t C l}_{2}(\mathrm{EtCN})_{2}\right] 483$ (Scheme 145 , reaction B), or the neutral platinum(IV) $\left[\mathbf{P t C l}_{4}(\mathbf{E t C N})_{2}\right]$ 484 (Scheme 145, reaction C). ${ }^{277}$ Compared to in solution, the reactions were accelerated by microwave irradiation and led to the formation of fused bicyclic oxadiazoline ligands when starting from cyclic nitrones. Longer reaction times were necessary when using acyclic compound 485 (Scheme 145, reaction $\mathrm{D}$ ). Interestingly, reacting nitrone and $\mathrm{EtCN}$ under platinum-free conditions did not yield the corresponding cyclic adduct. Similarly, the cycloaddition was described with cis- and trans- $\left[\mathbf{P t C l}_{2}(\mathbf{P h C N})_{2}\right]$ and led to the selective formation of cisor trans- $\left[\mathbf{P t C l}_{\mathbf{2}}\right.$ (oxadiazoline $\left.)(\mathbf{P h C N})\right]$, respectively, using different acyclic nitrones (485). ${ }^{278}$ Notably, the resulting complexes featuring the oxadiazoline ligand could be used for further reactions thanks to the lability of benzonitrile. Interestingly, subsequent reaction with a second equivalent of nitrone resulted in symmetrical or unsymmetrical trans$\left[\mathrm{PtCl}_{2}\left(\right.\right.$ oxadiazoline $\left.^{1}\right)\left(\right.$ oxadiazoline $\left.\left.^{2}\right)\right]$. In contrast, the reaction with the cis-isomer failed. A one-pot, microwave-assisted reaction was used in the direct synthesis of trans$\left.\left[\mathrm{PtCl}_{2} \text { (oxadiazoline }\right)_{2}\right]$ in only $1 \mathrm{~h}$ starting from a solution of acyclic nitrones and trans- $\left[\mathbf{P t C l}_{2}\left(\mathbf{R C H}_{2} \mathbf{C N}\right)_{2}\right](\mathrm{R}=$ $\left.\mathrm{CO}_{2} \mathrm{Me}, \mathrm{Cl}\right)$ in $\mathrm{DCM}^{279}$

A similar strategy in which the platinum activates the electrophilicity of the nitrile ligand was used starting from ketoximes 487 instead of nitrones. Microwave irradiation of the reaction mixture led to the addition of the oxime onto the nitrile to synthesize iminoacyl-platinum complexes 489 and 490 (Scheme 146). ${ }^{280}$ Compared to classical solution conditions, reactions were accelerated by microwave irradiation, leading to higher isolated yields without decreasing the selectivity. Both trans and cis isomers were formed during the process, but they could be separated by column chromatography. Selective formation of the trans isomer was possible when a stronger electron-withdrawing group such as a methyl ester was used. Contrastingly, the chloride derivative resulted in a mixture of cis and trans isomers.

Scheme 144. Microwave Preparation of Mono- and Dialkynyl Phosphino Platinum Complexes

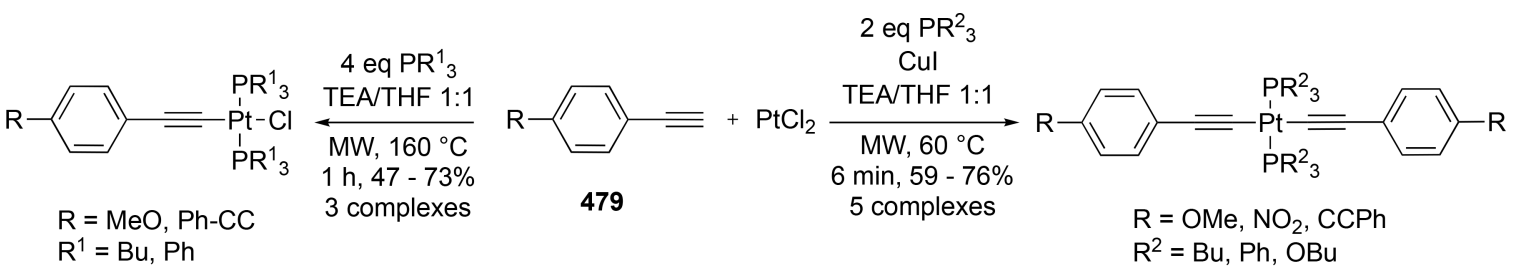

478 
Scheme 145. Microwave-Assisted [2+3] Cycloaddition of Nitrone to Platinum-Bound Organonitriles

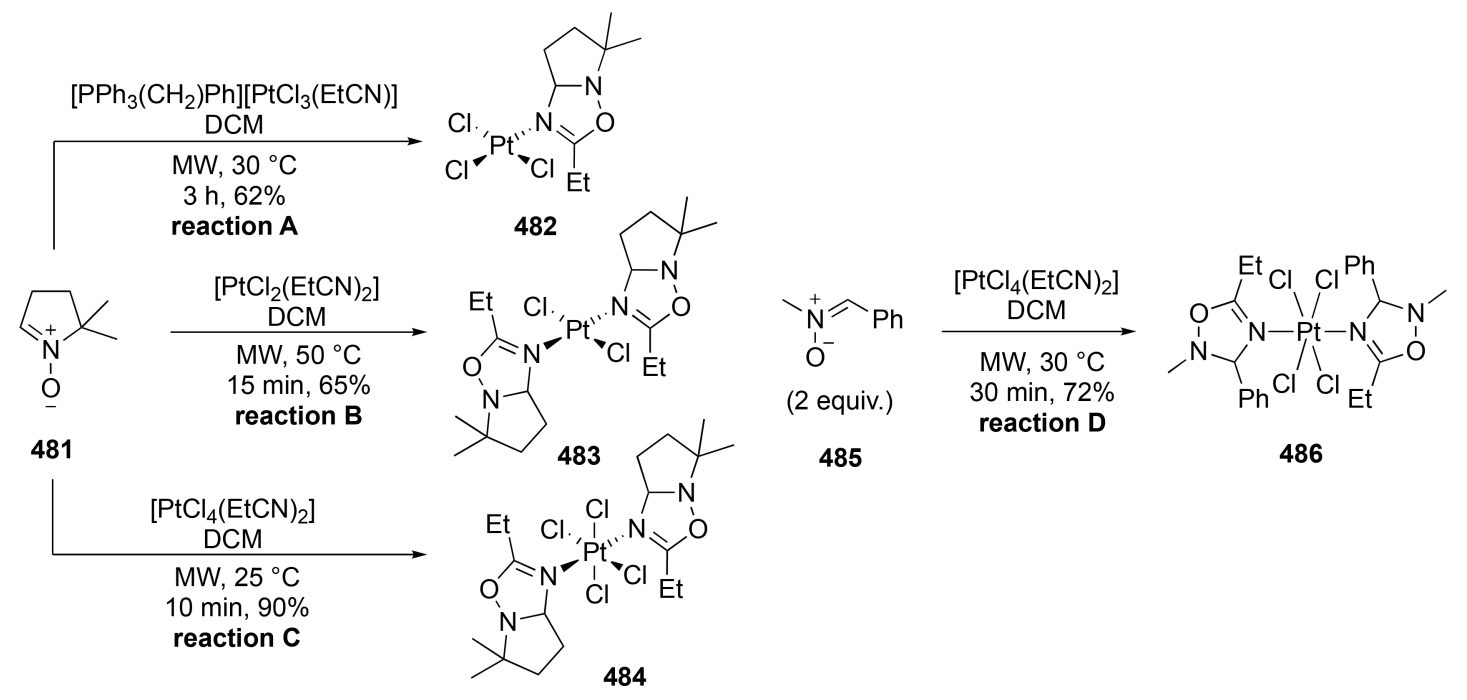

Scheme 146. Reaction of Ketoxime with Nitrile-Platinum Complexes under Microwave Irradiation

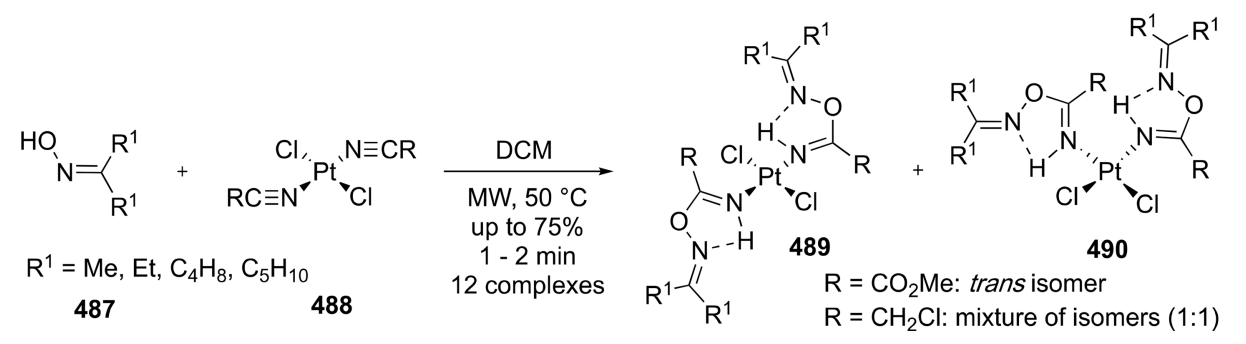

Similar cycloaddition was described for the formation of a tetrazole-containing platinum complex starting from 4fluorobenzonitrile 491 and a diazido-bis(triphenylphosphine) platinum complex (Scheme 147). ${ }^{281}$ The reaction conditions

Scheme 147. Microwave Synthesis of Tetrazolate-Platinum Complexes

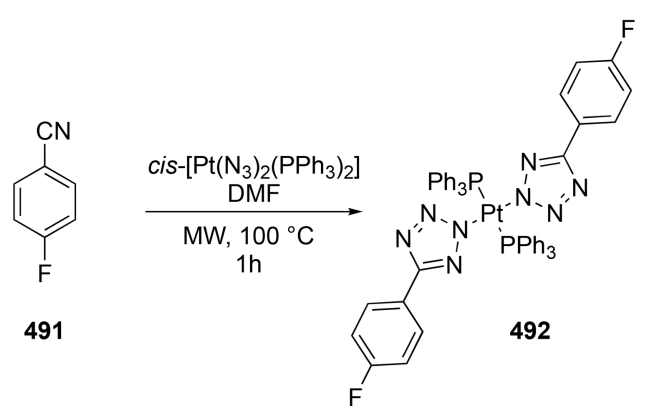

(MW, $100{ }^{\circ} \mathrm{C}, 1 \mathrm{~h}$ ) were then applied to the synthesis of trans$\left[\mathrm{Pt}\left(\mathrm{N}_{4} \mathrm{CR}\right)_{2}\left(\mathrm{PPh}_{3}\right)_{2}\right]$ complexes 492 starting from various nitriles $\left(\mathrm{RCN} ; \mathrm{R}=\mathrm{Me}, \mathrm{Et}, \mathrm{Pr}, \mathrm{Ph}\right.$, and $\left.\mathrm{C}_{6} \mathrm{H}_{4} \mathrm{Cl}\right){ }^{282}$ Interestingly, the reaction of cis- $\left[\mathbf{P t}\left(\mathbf{N}_{3}\right)_{2}\left(\mathbf{P P h}_{3}\right)_{2}\right]$ with propionitrile in solution produced the unexpected trans$\left[\mathbf{P t}(\mathrm{CN})(\right.$ 5-ethyltetrazolato $\left.)\left(\mathbf{P P h}_{3}\right)_{2}\right]$ complex resulting from an unsual $\mathrm{NC}-\mathrm{C}$ bond cleavage. This side product was completely undetected when the reaction was performed under microwave irradiation. Similarly, microwave irradiation of cis$\left[\operatorname{Pt}\left(\mathrm{N}_{3}\right)_{2}(2,2-\right.$ bipy $\left.)\right]$ with nitriles led to the synthesis of cis$\left[\mathbf{P t}\left(\mathrm{N}_{4} \mathrm{CR}\right)_{2}\left(\mathbf{2}, \mathbf{2}^{\prime}\right.\right.$-bipy $\left.)\right]$ complexes.
An interesting example of microwave-assisted reaction involved $[2+3]$ cycloaddition of azido-platinum complexes 493, featuring 1,3,5-triaza-7-phosphaadamantane (PTA) ligands, with organonitriles. This reaction yielded the corresponding tetrazolato complexes, which could liberate tetrazoles and the water-soluble cis- $\left[\mathbf{P t}(\mathrm{Cl})_{2}(\mathrm{PTA}-\mathrm{H})_{2}\right] \mathbf{C l}_{2}$ complex 495 in diluted $\mathrm{HCl}$ media (Scheme 148). ${ }^{283}$

Different pyridine and bipyridine complexes 496-497 were synthesized by microwave irradiation starting from a solution of platinum salt $\mathrm{K}_{2} \mathrm{PtCl}_{4}$ in an $\mathrm{EtOH} /$ water mixture (Scheme 149). ${ }^{284}$ Syntheses with 2-methylpyridine ligands failed probably due to the increased steric hindrance. A similar failure was observed upon microwave irradiation, with the 4,4'bis(2-morpholinoethoxy)-2,2'-bipyridine ligand. The desired complex could only be obtained by heating the reaction mixture at reflux for $24 \mathrm{~h}$ starting from $\left[\mathrm{PtCl}_{2}\right.$ (COD)]. $\mathrm{K}_{2} \mathrm{PtCl}_{4}$ was also used for the synthesis of $[\mathrm{PtCl}($ terpy $)] \mathbf{C l} \cdot$ $3 \mathrm{H}_{2} \mathrm{O} 498$ (terpy $=2,2^{\prime}, 2^{\prime \prime}$-terpyridine). Under classical reflux conditions, 498 was synthesized in $24-100 \mathrm{~h}$, whereas only 60 $\mathrm{s}$ was required under microwave irradiation, giving the desired complex in $47 \%$ yield. $^{91}$

Usually, cyclometalations of $\left[\mathrm{MX}_{3}\left(2,4^{\prime}\right.\right.$-bipy-Me $\left.)\right] 499(\mathrm{M}$ $=\mathrm{Pd}, \mathrm{Pt})$ give the corresponding $\left[\mathbf{M X}_{2}\left(\mathbf{2}, \mathbf{4}^{\prime}\right.\right.$-bipy-Me $\left.)\right]$ complexes (500) and require long reaction times to reach completion. In 1994, however, Castan et al. demonstrated that these reactions took only a few minutes under microwave irradiation (Scheme 150). ${ }^{285}$ Interestingly, palladium complexes were much more reactive than their platinum analogues, whereas chloride compounds were more reactive than their respective bromides. The reactions were also performed in the solid state by heating the sample at $140-200{ }^{\circ} \mathrm{C}$; however, 
Scheme 148. Microwave Synthesis of Water-Soluble Platinum Complexes
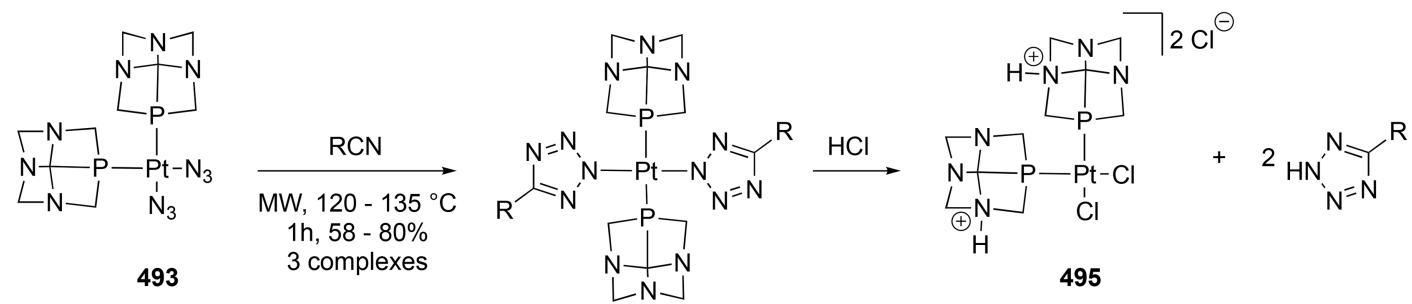

$\mathrm{R}=\mathrm{Ph}, 3$-pyridine, $4-\mathrm{ClC}_{6} \mathrm{H}_{4}$

494

Scheme 149. Microwave-Assisted Synthesis of PyridinePlatinum Complexes

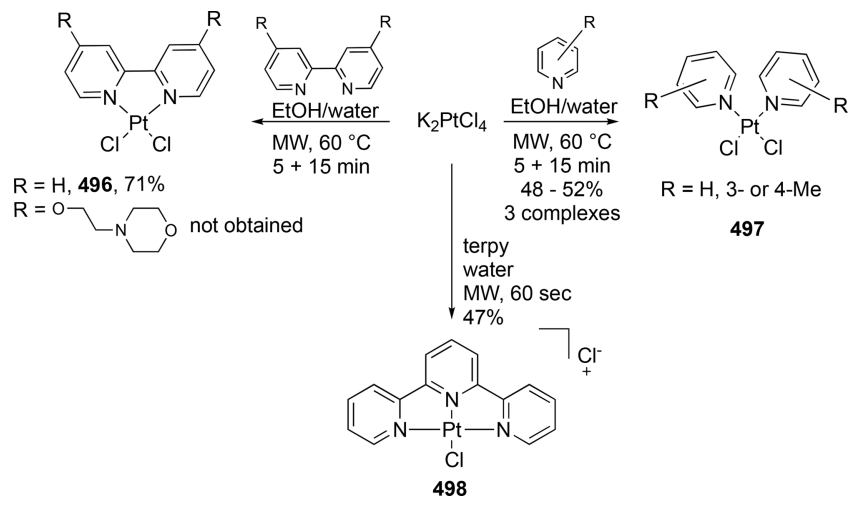

Scheme 150. Cyclometallation of $\left[\mathrm{MX}_{3}\left(2,4^{\prime}\right.\right.$-bipy-Me $\left.)\right]$
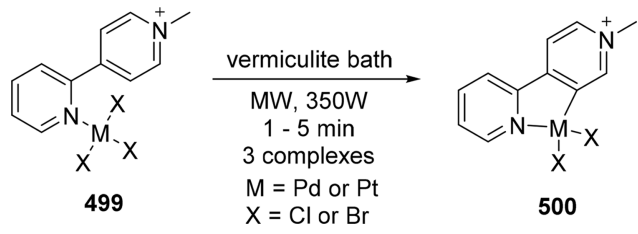

reactions using microwave irradiation were comparatively much faster ( $1 \mathrm{~h}$ instead of a few minutes).

Similarly, cyclometalated chloridoplatinum complexes 501504 containing benzo[ $h]$ quinolone, 4-methoxypyridine, 2phenylpyridine, and 2-(2'-thienyl)pyridine could be synthesized under microwave irradiation in only 1-6 min (Figure $8){ }^{286}$ Complexes were recovered in similar or lower yield

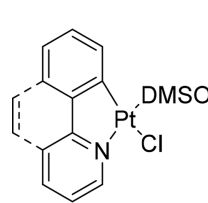

501

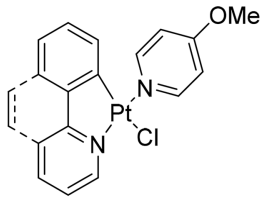

502

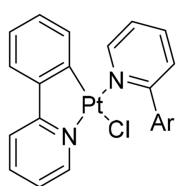

$\mathrm{Ar}=\mathrm{Ph}, \mathbf{5 0 3}$ $\mathrm{Ar}=$ thienyl, $\mathbf{5 0 4}$
Figure 8. Cyclometalated chloridoplatinum complexes prepared by microwave irradiation.

compared to using conventional heating. This could be explained by the rapid decomposition of the platinum salt to black $\mathrm{Pt}^{0}$, which was observed during synthesis at high temperatures.

The reaction of metal chlorides $\mathrm{MCl}_{2}(\mathrm{M}=\mathrm{Pd}, \mathrm{Pt})$ with Schiff base ligand $\mathbf{5 0 5}$ has been shown to form $\left[\mathbf{M}(\mathbf{5 0 5})_{2} \mathbf{C l}_{2}\right]$ or, in the presence of $\mathrm{NH}_{4} \mathrm{OH},\left[\mathrm{ML}_{2}\right]$ 506. Both types of complexes could be formed under classical reflux conditions in a few hours. When syntheses were performed under microwave irradiation, reaction times were reduced to 5-7 min (Scheme 151). ${ }^{287}$ Both complexes and ligands were screened for their

Scheme 151. Microwave Synthesis of Schiff Base Palladium and Platinum Complexes

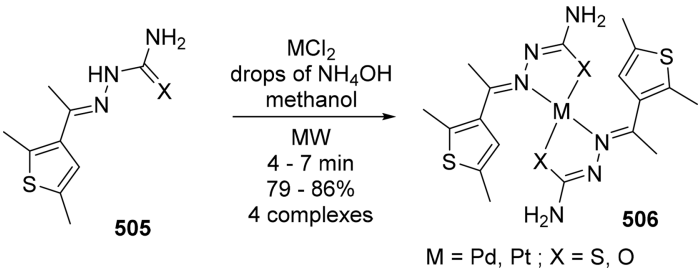

antimicrobial properties. Interestingly, the complexes showed better antimicrobial and antiamoebic activities than the ligand itself and also inhibited the growth of fungi and bacteria (Entamoeba histolytica).

Palladium complexes bearing NHC ligands could also benefit from microwave-assisted synthesis. ${ }^{28}$ Thus, PEPPSItype complexes $\left[\mathbf{P d C l}_{2}(\mathbf{N H C})(3\right.$-chloropyridine) $] \quad 507$ (Scheme 152, reaction A) and $[\mathbf{P d C l}(\mathbf{N H C})($ acac $)] 508$

Scheme 152. Microwave-Assisted Synthesis of NHCPalladium Complexes

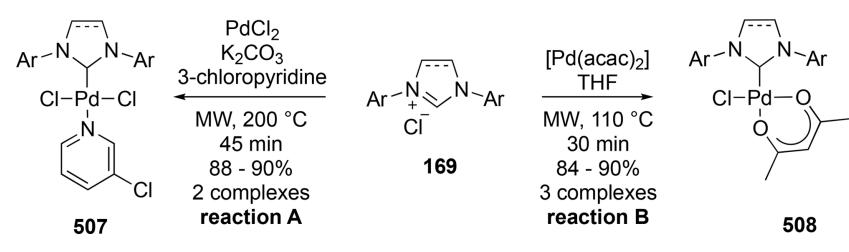

(Scheme 152, reaction B) were synthesized with a significant reduction in the reaction time compared to classical solution conditions (20-88 times faster). This protocol was then applied to the synthesis of $[\mathbf{P d C l}(\mathrm{IPr})(\mathrm{acac})]$ complexes (IPr $=1,2$-bis $(2,6$-diisopropylphenyl)imidazol-2-ylidene) on a 5 $\mathrm{mmol}$ scale $(2.76 \mathrm{~g})$. The complexes were obtained in only 30 $\min$.

A new catalyst for different cross-coupling reactions was synthesized under microwave irradiation by $\mathrm{Tu}$ et al. ${ }^{289}$ Interestingly, the complex was prepared from cheap and commercially available precursors, namely, imidazolium salt 509 and palladium acetate, in DMSO under irradiation at 160 ${ }^{\circ} \mathrm{C}$ for $25 \mathrm{~min}$ (Scheme 153). Despite this, complex $\mathbf{5 1 0}$ was recovered in moderate yield. Catalytic properties of the 
Scheme 153. Microwave Synthesis of a Pyridine-Bridged Bisbenzimidazolylidene CNC Pincer Palladium Complex

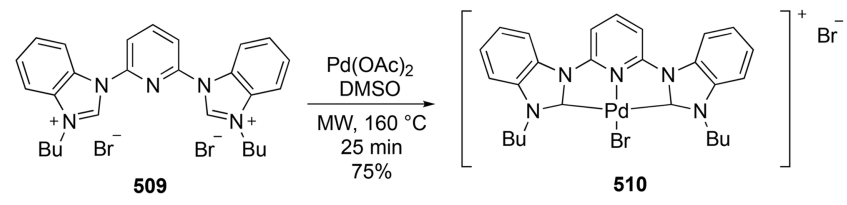

pyridine-bridged bisbenzimidazolylidene CNC pincer palladium complex $\mathbf{5 1 0}$ were demonstrated in Mizoroki-Heck and Suzuki-Miyaura cross-couplings at catalyst loadings as low as 0.1-1 ppm. Notably, the catalyst was thermally stable under air and moisture.

Similarly, an original prolinol-based chiral NCN pincer palladium complex $\mathbf{5 1 2}$ was quickly and efficiently synthesized under microwave irradiation, via an insertion of $\mathrm{Pd}^{0}$ into a $\mathrm{C}-$ $\mathrm{Br}$ bond (Scheme 154). ${ }^{290}$ Under standard conditions,

Scheme 154. Microwave Synthesis of a NCN Pincer Palladium Complex

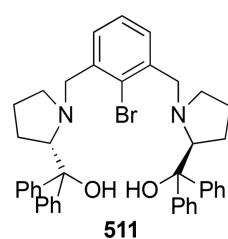

511
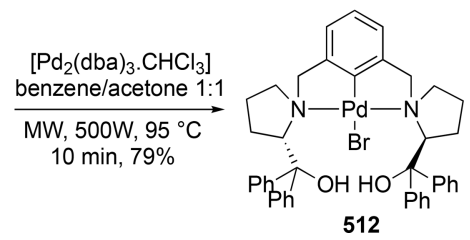

512 complex $\mathbf{5 1 2}$ was obtained in $44 \%$ yield after $16 \mathrm{~h}$ of thermal heating at $60{ }^{\circ} \mathrm{C}$. The resulting bromide derivative could be transformed by metathesis reaction to the corresponding $\mathrm{BF}_{4}$ and $\mathrm{PF}_{6}$ products. Both complexes were evaluated in an aldol condensation reaction between methyl isocyanoacetate and aromatic aldehyde, efficiently yielding cis-oxazolines with good regio- and stereoselectivity.

Microwave irradiation was also used to synthesize different porphyrin metal complexes (517). Complexes of $\mathrm{Ni}, \mathrm{Pd}$, and $\mathrm{Pt}$ were recovered easily within a few minutes and in yields higher than $70 \%$, if not quantitative (Scheme 155). ${ }^{291}$ Using

Scheme 155. Microwave-Assisted Synthesis of Metalloporphyrins

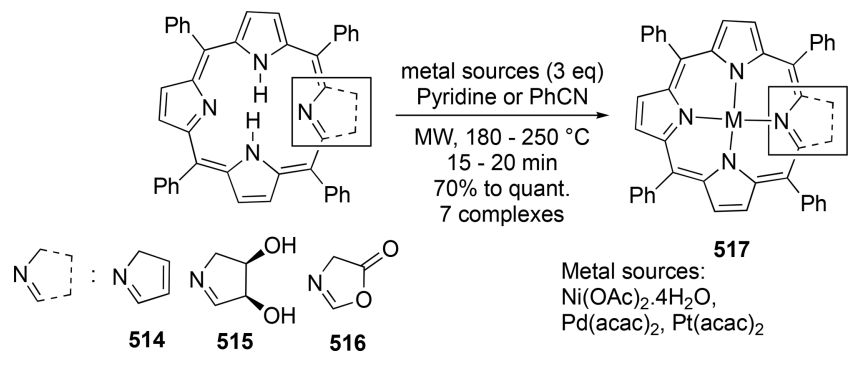

the diol-containing porphyrin ligand 515, various nickel and palladium byproducts arising from the diol oxidation were recovered. Despite this, the corresponding nickel complex was recovered in $70 \%$ yield after workup. Unfortunately, in the case of palladium, only 50\% conversion could be obtained. Notably, syntheses were also performed with platinum sources, but corresponding complexes were difficult to form. However, when reaction temperatures were increased to $250{ }^{\circ} \mathrm{C}$ and benzonitrile was used instead of pyridine as the solvent, the desired complexes were isolated in quantitative yields. Classical solution methods were much slower than the microwaveassisted method (reaction times up to days). Nevertheless, classical solvent-based conditions led to a more selective complex synthesis featuring the diol derivative with less nickel and palladium byproducts, thus demonstrating that the byproduct formation was accelerated under microwave irradiation.

\subsection{Ultrasounds for the Synthesis of a Nickel Complex}

There is one publication reporting the use of ultrasound for the synthesis of group 10 organometallic complexes. In this study, nickel acetate and KbpzB 518 (potassium bispyrazolyl borate) were reacted under ultrasound for $1 \mathrm{~h}$, leading to the formation of nanostructures of $\left[\mathrm{Ni}(\mathrm{bpzB})_{2}\right]_{2} 519$ in $66 \%$ yield (Scheme 156). ${ }^{292}$ The nanostructure was characterized by SEM

\section{Scheme 156. Ultrasound Synthesis of Nanostructured $\left[\mathrm{Ni}(\mathrm{bpzB})_{2}\right]_{2}$}
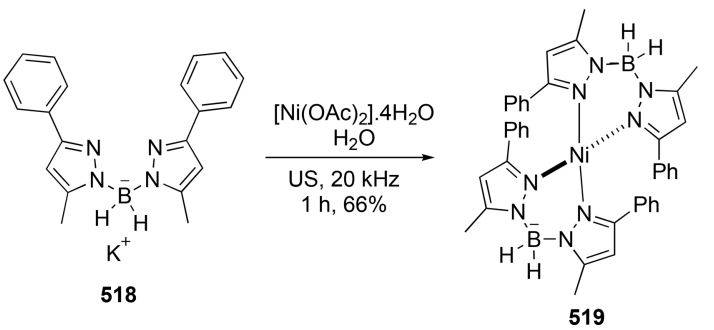

(scanning electron microscopy), PXRD, IR, and elemental analysis. Interestingly, without the use of ultrasound, 519 was no longer recovered as nanostructures but as single crystals. Analyses revealed that individual molecules interacted with each other by directional intramolecular interactions, creating 3D supramolecular frameworks.

\subsection{Continuous Flow Synthesis of Nickel Complexes}

To date, only one example of continuous flow chemistry applied to the synthesis of complexes involving a group 10 metal has been reported. New square-planar nickel(II)salphen complexes $\mathbf{5 2 1}$ were synthesized by Rakers et al. in 2018 using a continuous flow approach (Scheme 157). ${ }^{293}$

\section{Scheme 157. Continuous Flow Synthesis of Nickel Complexes}
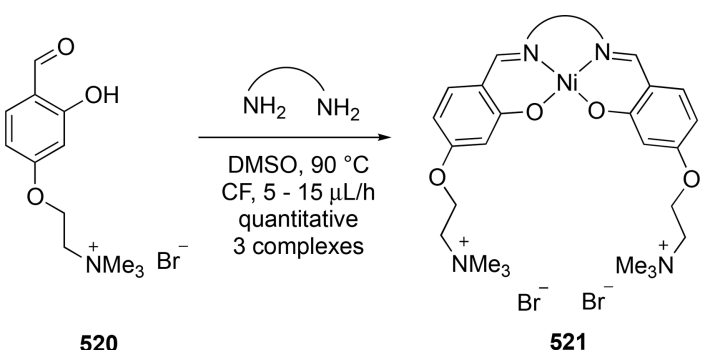

Complexes were obtained in two steps, synthesis of the ligand through condensation of a diamine (ethylene diamine, $o$ phenylene diamine, or 2,3-diaminonaphthalene) and the aldehyde $\mathbf{5 2 0}$ followed by coordination of the metal. A onepot methodology ultimately facilitated the isolation of the same complexes in quantitative yields, and no purification was required. Interestingly, two of these complexes have been 


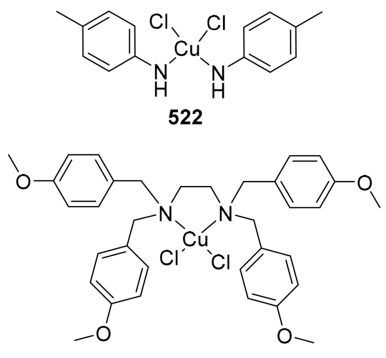

523

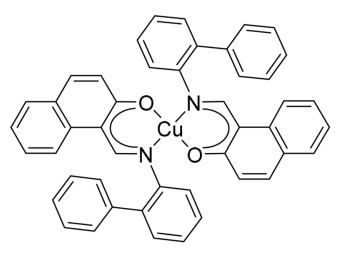

524

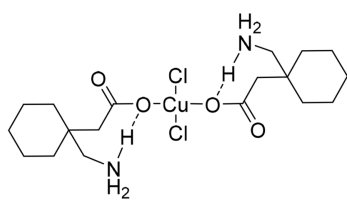

525

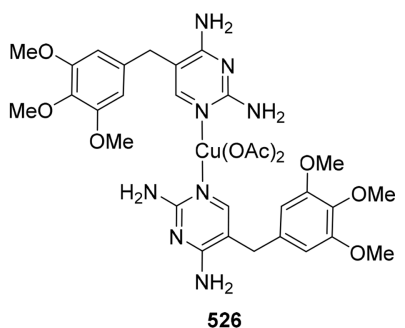

526

Figure 9. Copper complexes with $\mathrm{N}$-coordinated ligands.

found to be very good G-quadruplex DNA binders and displayed high selectivity for duplex DNA.

\section{GROUP 11}

Copper, silver, and gold complexes play an important role in organic as well as organometallic chemistry. ${ }^{294}$ Also known as coinage metals, their utility in catalysis is well documented, although there is still room for development of new reactivities. Their corresponding organometallic complex can also possess therapeutic properties, such as antifungal or antibacterial activities. Numerous groups are working on their syntheses using nonconventional methods.

\subsection{Mechanosynthesis and Coinage Metals}

In 2011, Sun and co-workers described the first dimerization of a copper metal complex $\left(\left[\mathrm{Cu}\left(\mathrm{NH}_{3}\right)_{3}(\mathrm{~L})\right] \cdot\left(\mathrm{H}_{2} \mathrm{O}\right)_{0.66}, \mathrm{H}_{2} \mathrm{~L}=\right.$ 2,2'-(1,2-phenylenebis(methylene))bis(sulfanediyl)dibenzoic acid) by mechanical activation. ${ }^{295}$ The synthesis was carried out in a steel vessel in a vibratory ball mill operated at $20 \mathrm{~Hz}$ for $30 \mathrm{~min}$. Dimerization was also performed under classical thermal activation for $15 \mathrm{~min}$ in an oven at $176^{\circ} \mathrm{C}$. The color of the complex changed from green to blue as the transformation involved a change in the coordination geometry of the copper ion. This color change indicated that the transformation may have potential application in sensing devices.

In 2009, the Bowmaker group grinded copper(I) thiocyanate and ethylenethiourea (etu) in stoichiometric quantities to form $\left[\mathrm{Cu}(\mathrm{SCN})(\text { etu })_{2}\right]$ after accelerated aging at $70{ }^{\circ} \mathrm{C}$ for 2 h. ${ }^{296}$ The reaction could be monitored $e x$ situ by IR thanks to the $\nu(\mathrm{CN})$ variations in the thiocyanate ligand. The addition of a few drops of water as a grinding assistant was necessary to obtain a complete reaction. In only $2 \mathrm{~h}$, the desired $\left[\mathrm{Cu}(\mathrm{SCN})(\mathrm{etu})_{2}\right]$ complex could be quantitatively isolated. Notably, when performed in solution, a poor conversion to a mixture of $\left[\mathrm{Cu}(\mathrm{SCN})(\text { etu })_{2}\right] /[\mathbf{C u}(\mathrm{SCN})($ etu $)]$ was obtained, thus demonstrating that mechanochemistry was more efficient and selective than solution conditions. The 1:1 adduct $[\mathrm{Cu}(\mathrm{SCN})($ etu $)]$ was prepared following the same method. Both reagents were mixed in a mortar and pestle and in stoichiometric amounts in the presence of water and heated at $70{ }^{\circ} \mathrm{C}$ overnight. Water was crucial for the reaction and likely helps diffusion and therefore the reagents to react. Interestingly, the formation of $[\mathbf{C u}(\mathbf{S C N})($ etu $)]$, starting from a $1: 1$ mixture of $\mathrm{CuSCN} / \mathrm{etu}$, undergoes rapid formation of
$\left[\mathrm{Cu}(\mathrm{SCN})(\mathrm{etu})_{2}\right]$, which slowly reacts further with remaining CuSCN.

The same group also described the mechanosynthesis of $\left[\mathrm{Cu}(\mathrm{SCN})\left(\mathrm{PCy}_{3}\right)_{x}\right](x=1,2),\left[\mathrm{Cu}(\mathrm{SCN})\left(\mathrm{PCy}_{3}\right)(\mathrm{py})\right]$, and $\left[\mathbf{C u}(\mathrm{SCN})\left(\mathbf{P}(\boldsymbol{o} \text {-tol })_{3}\right)(\mathrm{ACN})\right]{ }^{297}$ The complexes were obtained by grinding reagents (bulky tertiary phosphine, pyridine, and $\mathrm{CuSCN}$ ) in a mortar and pestle with acetonitrile as liquid additive. Under classical solution conditions care was taken to exclude air moisture, due to the possible oxidation of the phosphine, and syntheses were usually performed in a glovebox. Mechanochemical treatments enabled this problem to be entirely circumvented. The reaction was complete after only a few minutes, whereas several hours was necessary in solution. Obtained yields were excellent $(>95 \%)$ and better than those in solution $(<80 \%)$.

In a similar manner, grinding copper halides $\left(\mathrm{CuCl}_{2} \cdot 2 \mathrm{H}_{2} \mathrm{O}\right.$, $\mathrm{CuI}$, and $\left.\mathrm{CuBr}_{2}\right)$, pyridine, and iodine in a mortar and pestle in air provided $\left[\mathrm{CuX}_{2}(\mathbf{P y})_{4}\right] \cdot \mathbf{2 P y}\left(\mathrm{X}=\mathrm{I}, \mathrm{I}_{3}, \mathrm{IBr}, \mathrm{I}(\mathrm{NCS})\right)$ and $\left[\mathrm{H}_{2} \mathrm{O}(\mathrm{Py})_{4} \mathrm{CuClCu}(\mathrm{Py})_{4}\right]\left(\mathrm{I}_{3}\right)_{3} \cdot \mathrm{H}_{2} \mathrm{O}$ complexes. ${ }^{298}$ Pyridine was used as a reagent but also as a liquid additive. Pure copper(II)-iodo complexes were also prepared from copper(I) iodide in the presence of pyridine and iodine. This method was applied to prepare mixed iodo/(pseudo)halo analogues and perform a five-component redox synthesis in excellent yields $(>90 \%)$.

In 2008, the same authors used mechanochemistry to perform a ligand dissociation reaction. ${ }^{299}$ Ground in an agate mortar and pestle, $\left[\mathbf{C u}(\mathbf{S C N})\left(\mathbf{P P h}_{3}\right)(\mathbf{P y})_{2}\right]$ with acetonitrile led to the formation of $\left[\mathbf{C u}(\mathbf{S C N})\left(\mathbf{P P h}_{3}\right)(\mathbf{P y})\right]$ with loss of one pyridine within a few minutes. Interestingly, no reaction was observed without milling. In solution, while heating at 120 ${ }^{\circ} \mathrm{C}$, the reaction led to a mixture of the expected product and $\mathrm{Cu}(\mathrm{SCN})$ as side product. This example clearly shows that the application of grinding enables isolation of products otherwise inaccessible under standard solution conditions.

It is also possible to mechanosynthesize dichlorobis $(p$ toluidine) copper(II) 522 by grinding $\mathrm{CuCl}_{2} \cdot 2 \mathrm{H}_{2} \mathrm{O}$ ( 1 equiv) and $p$-toluidine ( 2 equiv) for five minutes. The analytically pure product was obtained in $96 \%$ yield (Figure 9). ${ }^{229}$ Using a similar strategy with a bidentate ligand, a mechanochemical formation of $\left[\left(\mathrm{CuCl}_{2}\right)\left(N, N, N^{\prime}, N^{\prime}\right.\right.$-tetra-p-methoxybenzylethylenediamine $)] \cdot 2\left(\mathbf{H}_{2} \mathbf{O}\right) \mathbf{5 2 3}$ was described in $2014 .^{300}$ The bidentate ligand was first protonated by $\mathrm{HCl}$ and then ground with $\mathrm{CuCl}_{2} \cdot \mathrm{H}_{2} \mathrm{O}$ for $1 \mathrm{~min}$ in a mortar and pestle to produce $\left[\mathrm{H}_{2} \mathbf{L}\right]^{2+}\left[\mathrm{CuCl}_{4}\right]^{2-}$ in quantitative yield. Subsequently, the reaction mixture was ground with $\mathrm{KOH}$ in a $1: 2$ ratio to 
Scheme 158. Mechanosynthesis of a Copper-Salen Complex

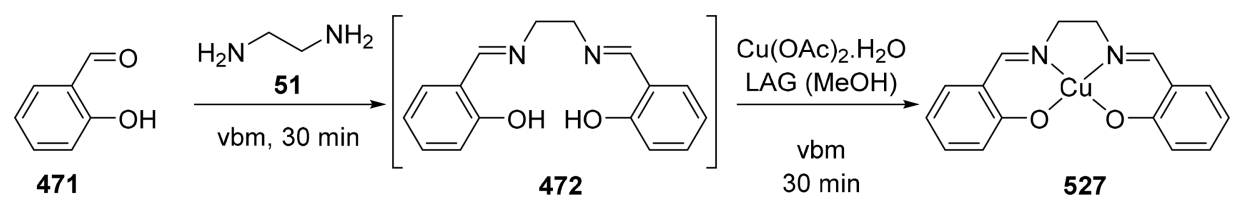

carry out the dehydrochlorination and obtain 523. Neat grinding was unsuccessful, but addition of a few drops of methanol led to complete conversion after only a few minutes of milling. Similarly, copper(II) complex 524 featuring 1-[(2biphenylamino)methylene]naphthalene-2 $(1 H)$-one ligands was synthesized by grinding the organic ligand (2 equiv) with $\mathrm{Cu}(\mathrm{OAc})_{2} \cdot \mathrm{H}_{2} \mathrm{O}$ ( 1 equiv) and triethylamine as a liquid additive. ${ }^{232}$ Grinding was performed in a $10 \mathrm{~mL}$ stainless steel jar using a vibratory ball-mill Retsch MM200 operated at 25 Hz. The copper complex was obtained after only $50 \mathrm{~min}$ in quantitative yield. Under reflux conditions $(\mathrm{MeOH} / \mathrm{ACN} 1: 1$, $2 \mathrm{~h}$ ) the desired product was obtained in $80 \%$ yield.

In 2008, the Braga group described the mechanosynthesis of $\left.\left[\mathbf{C u C l}_{2} \text { (gabapentin) }\right)_{2}\right] \mathbf{5 2 5}$ by manual grinding of solid amorphous gabapentin and $\mathrm{CuCl}_{2} \cdot 2 \mathrm{H}_{2} \mathrm{O}$ in an agate mortar and pestle. ${ }^{301}$ The desired complex was obtained in quantitative yield after only $5 \mathrm{~min}$ of grinding. The amino acid gabapentin is a neuroleptic drug, and the use of coordination complexes of an active pharmaceutical ingredient may open up new drug delivery routes. Following this concept, milling of $\mathrm{Cu}(\mathrm{OAc})_{2} \cdot \mathrm{H}_{2} \mathrm{O}$ and trimethoprim for $20 \mathrm{~min}$ in a stoichiometric quantity provided $\left[\mathrm{Cu}(\mathrm{OAc})_{2}(\mathrm{TMP})_{2}\right] 526$ in $80 \%$ yield. ${ }^{268}$ The reaction time was drastically reduced since 2 $\mathrm{h}$ was necessary under reflux.

Salen-type copper complex 527 could be obtained readily from the appropriate aldehyde and diamine using ball milling (Scheme 158). ${ }^{270}$ Intermediate formation of the ligand $\mathbf{4 7 2}$ required $30 \mathrm{~min}$ of milling, and copper acetate was added directly into the milling jar, with methanol as a grinding assistant, to quantitatively afford the desired copper-salen complex. Contrary to observations with nickel (Scheme 139), the final complex was analytically identical to that prepared using classical solution techniques.

In 2014, the Kato group described the mechanosynthesis of three different luminescent copper iodide complexes, [CuI$\left.\left(\mathrm{PPh}_{3}\right)_{2}(\mathrm{~L})\right] 528$ ( $\mathrm{L}=$ isoquinoline, 1,6-naphthyridine, and pyridine) (Scheme 159). ${ }^{302}$ These complexes were formed by grinding copper(I) iodide, triphenylphosphine, and various $\mathrm{N}$ heteroaromatic ligands $\mathbf{5 2 9}$ in an agate mortar and pestle for a few minutes. The products were obtained in yields between 63 and $85 \%$ without any impurities. Notably, the reactions were

Scheme 159. Mechanosynthesis of Luminescent Copper Complexes
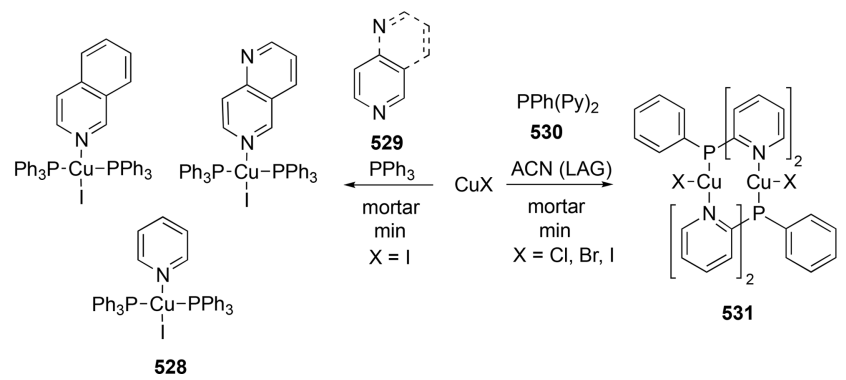

531 not complete within less than 3 equiv of the N-heteroaromatic ligand. In 2016, the same authors also described the mechanosynthesis of highly luminescent $\mathrm{Cu}(\mathrm{I})$ dinuclear complexes $\left.\left[\mathrm{Cu}_{2} \mathbf{X}_{2} \text { (dpypp }\right)_{2}\right] 531(\mathrm{X}=\mathrm{Cl}, \mathrm{Br}, \mathrm{I}$, dpypp = 2,2'-(phenylphosphinediyl)dipyridine 530), using a mortar and pestle with yields between $63 \%$ and $80 \% .{ }^{303}$ $\left[\mathbf{C u}_{2} \mathbf{I}_{2}(\mathbf{d p y p p})_{2}\right]$ could also be obtained with addition of a liquid assistant, starting from $\left[\mathrm{Cu}_{2}(\mathbf{A C N})_{2}(\mathrm{dpypp})_{2}\right]\left(\mathrm{BF}_{4}\right)_{2}$ and $\mathrm{KI}$ in equimolar quantities for $3 \mathrm{~min}$ (60\% yield). Among the different solvents tested as an assistant, acetonitrile and benzonitrile were the best choices mainly due to the high solubility of copper in both solvents.

In 2017, the Lamaty group reported the synthesis of $N, N$ diaryl NHC copper complexes $\mathbf{5 3 2}$ by transmetalation in a ball mill (Scheme 160). ${ }^{262}$ Starting from the corresponding NHC

\section{Scheme 160. Synthesis of NHC Copper Complexes by} Transmetalation or Direct Metalation

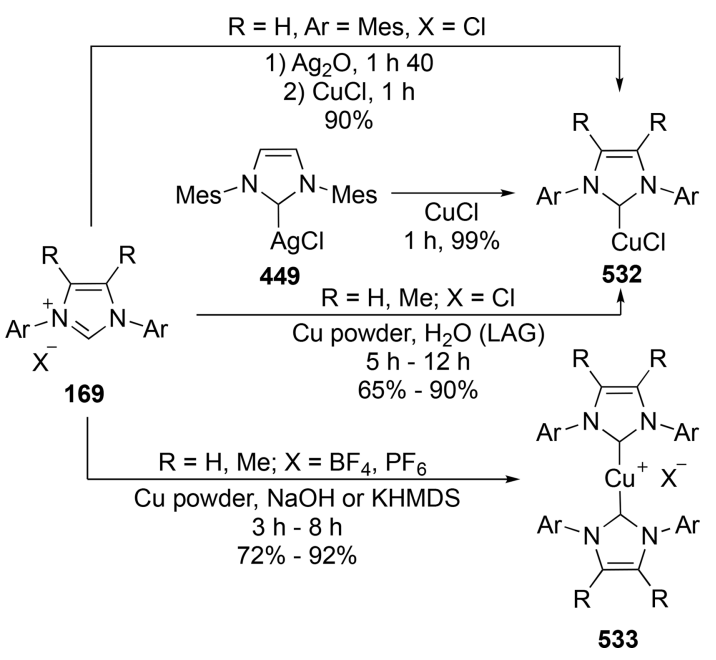

silver species 449, 532 was obtained in 99\% yield using vibratory ball-mill Retsch MM200 at $25 \mathrm{~Hz}$ in only $1 \mathrm{~h}$. A onepot, two-step synthesis, involving metalation of imidazolium salt 169 with $\mathrm{Ag}_{2} \mathrm{O}$ and transmetalation with $\mathrm{CuCl}$, allowed the isolation of the corresponding $[\mathrm{CuCl}(\mathrm{NHC})]$ complex in $90 \%$ yield after 160 min of milling. N,N-Diaryl NHC copper complexes could also be obtained via direct metalation of various imidazolium salts $\mathrm{NHC} \cdot \mathrm{HX}\left(\mathrm{X}=\mathrm{Cl}, \mathrm{BF}_{4}\right.$ and $\left.\mathrm{PF}_{6}\right)$ using copper powder in a planetary ball-mill Retsch PM100 at $450 \mathrm{rpm}$ (Scheme 160). ${ }^{304}$ Due to highly efficient mixing, the reaction was performed between gas (dioxygen from air), solid (copper(0) and imidazolium salt), and liquid (water) in a very short time and gave corresponding copper(I) complexes $\mathbf{5 3 2}$ and $\mathbf{5 3 3}$ in yields of up to $97 \%$. Importantly, the quantity of $\mathrm{O}_{2}$ could be reduced to nearly stoichiometric amounts, while keeping the same efficiency. Additionally, mechanosynthesis outperformed classical syntheses under reflux in terms of reaction time and yield, in particular for highly challenging 
Scheme 161. Different Silver Chloride Complexes with Ethylenethiourea
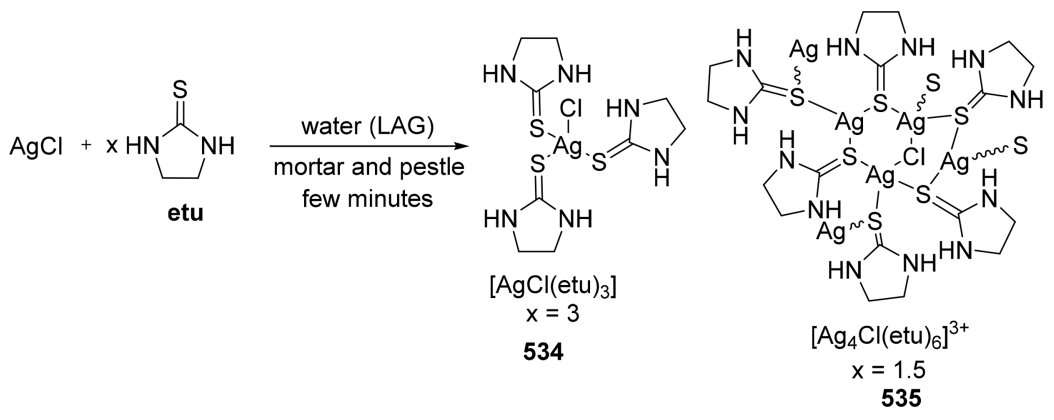

complexes such as homoleptic copper complexes $\mathbf{5 3 3}$ featuring noncoordinating anions.

Silver is one of the less expensive noble metals, and corresponding complexes are highly developed for different applications, such as catalysis, in particular for activation of alkynes. ${ }^{305}$ Numerous silver salts and complexes exhibited biological properties (antimicrobial, ${ }^{306}$ anticancer, ${ }^{307}$ or antiinflammatory $^{308}$ ).

Bowmaker et al. used mechanochemistry, and more specifically grinding using a mortar and pestle, to quickly evaluate the stability and formation of silver complexes featuring various thioureas $(\mathbf{x}) \mathbf{t u}$ as ligands (tu = thiourea, detu $=N, N^{\prime}$-diethylthiourea, and ettu $=N$-ethylthiourea) in highly concentrated mixtures. In all of these studies, liquidassisted grinding was necessary to obtain satisfactory reaction rates. Manual grinding of $\left[\mathrm{Ag}_{4} \mathrm{Cl}(\text { etu })_{6}\right] \mathbf{C l}_{3} \mathbf{5 3 5}$, as a $1: 1.5$ $\mathrm{AgCl} /$ etu complex, with 1.5 equiv of ethylenethiourea and a few drops of water provided $\left[\mathrm{AgCl}(\text { etu })_{3}\right] 534$ (Scheme 161). ${ }^{309}$ When lower amounts of etu were used, a mixture composed of starting materials and $\mathbf{5 3 4}$ was obtained, as witnessed by IR spectroscopy. This confirmed that [AgCl(etu) ${ }_{2}$ ] was unstable with respect to the other complexes. Since the complex $[\operatorname{AgI}($ etu $)]$ was found to be highly unstable in water, the authors subsequently investigated the reaction of $\left[\operatorname{AgI}(\text { etu })_{0.5}\right]$ with varying amounts of etu. IR analysis demonstrated that $\left[\mathrm{AgI}(\mathrm{etu})_{2}\right]$ could be formed exclusively and that $[\operatorname{AgI}(\mathbf{e t u})]$ and $\left[\operatorname{AgI}(\text { etu })_{3}\right]$ were too unstable to be observed. The same authors further extended the knowledge of the mechanochemistry of silver halides with thiourea. ${ }^{310}$ $[\mathbf{A g X}(\mathbf{t u})],\left[\mathbf{A g X}(\mathbf{t u})_{3}\right]$, and $\left[\mathbf{A g X}(\mathbf{t u})_{4}\right](\mathrm{X}=\mathrm{Cl}, \mathrm{Br}, \mathrm{I})$ could be obtained selectively using various silver:tu ratios. Use of mono- and disubstituted ureas led to the unique formation of $\left[\operatorname{AgX}(\text { ettu })_{3}\right]$ and $\left[\operatorname{AgX}(\text { detu })_{3}\right]$, respectively. This difference in behavior was assigned to the reduction of hydrogen bonding strength upon urea $\mathrm{N}$-substitution. A similar study was performed using silver(I) thiocyanate as the starting material. ${ }^{311}$ The mechanochemical method enabled the verification of the findings of the stoichiometric reaction of $\mathrm{AgSCN}$ with etu, that $[\mathrm{AgSCN}($ etu)] was not stable enough to be observed. Instead, a mixture of $\left[\mathrm{AgSCN}(\text { etu })_{2}\right] /[\mathrm{AgSCN}$ $\left.(\text { etu })_{0.5}\right]$ was obtained. Interestingly, $\left[\operatorname{AgSCN}(\text { etu })_{0.5}\right]$ was never observed before, even when performing the reactions in solution. When silver nitrate was used as a starting material, the solid adducts $\left[\mathrm{AgNO}_{3}(\mathbf{t u})_{x}\right]$ could be synthesized $(x=1,1.5$, $1.53,3) .{ }^{291}$ Importantly, the mechanochemical procedure allowed the suppression of the undesired side reactions, which occurred in solution in the case of $\left[\operatorname{AgNO}_{3}(\mathbf{t u})\right]$, to access new $\mathrm{Ag} / \mathrm{tu}$ stoichiometries (e.g., 15:23).

In 2011, the same group reported the mechanosynthesis of $\left[\mathbf{A g}\left(\mathbf{H C O}_{3}\right)\left(\mathbf{P P h}_{3}\right)_{2}\right]$ grinding $\mathrm{Ag}_{2} \mathrm{CO}_{3}, \mathrm{PPh}_{3}, \mathrm{CO}_{2}$, and $\mathrm{H}_{2} \mathrm{O}$ with ethanol as a liquid assistant with a mortar and pestle. ${ }^{312}$ Interestingly, the formation of the bicarbonate ligand came from atmospheric $\mathrm{CO}_{2}$ and $\mathrm{H}_{2} \mathrm{O}$. Similarly, $\left[\mathrm{Ag}\left(\mathrm{H}_{2}\right.\right.$ cit $)$ $\left.\left(\mathbf{P P h}_{3}\right)_{2}\right] \cdot \mathbf{E t O H}, \quad\left[\left(\mathbf{A g}\left(\mathbf{P P h}_{3}\right)_{2}\right)_{2}\left(\mathbf{H}_{2} \mathrm{cit}\right)\right]$, and $\left[\mathrm{Ag}\left(\mathrm{H}_{2} \mathrm{cit}\right)\right.$ $\left.\left(\mathbf{P P h}_{3}\right)_{3}\right] \quad\left(\mathbf{H}_{2} \mathbf{c i t}=\right.$ dihydrogencitrate $)$ were formed by mechanosynthesis using a one-pot two-step procedure, in which the liquid assistant was purposefully different in the two steps. ${ }^{313}$ The choice of assistant was according to the solubility of the reagents and products. First, silver oxide and citric acid were ground with a small quantity of water, followed by addition of triphenylphosphine and a small amount of ethanol to yield an off-white paste. The ratio of all reagents was varied as to obtain different complexes. This procedure enabled reaction times to be reduced to a minimum. The mechanosynthesis of trisilver(I) citrate was also reported by grinding silver(I) oxide and citric acid in a 3:2 ratio by water-assisted grinding.

Syntheses of different NHC silver complexes 536 and 537 were developed by the Lamaty group. N,N-Dialkyl NHC silver halide complexes were formed by mixing an imidazolium salt and silver oxide in a stainless-steel jar using a vibratory ball-mill Retsch MM200 at $25 \mathrm{~Hz}$ (Scheme 162, reaction A). ${ }^{314}$ Eleven

Scheme 162. Mechanosynthesis of NHC Silver Complexes

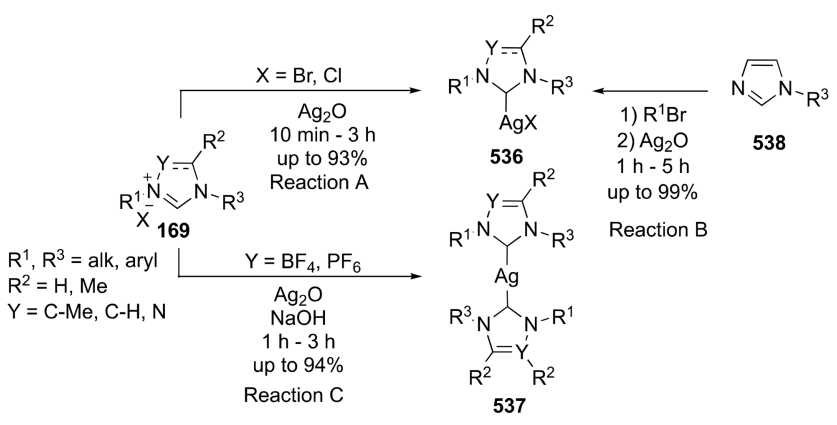

complexes were prepared in yields of up to $93 \%$ with milling times between 10 and 150 min featuring different alkyl groups on the ligand. Comparison with literature conditions showed that the ball-mill approach has a real positive effect in terms of reaction times and yields and gives access to unprecedented complexes. A one-pot, two-step approach from imidazole $\mathbf{5 3 8}$ enabled avoidance of imidazolium salt intermediate isolation, while keeping the same overall efficiency (Scheme 162, reaction $\mathrm{B}$ ). To generalize the method, authors described the synthesis of $N, N$-diaryl NHC silver halide complexes. ${ }^{262}$ Due to the lower reactivity of $N, N$-diaryl imidazolium salts, the milling frequency was increased to $30 \mathrm{~Hz}$; however, a short reaction time of 1.5 to $3 \mathrm{~h}$ was kept, even for the synthesis of 
Scheme 163. Synthesis and Transformation of NHC Gold Complexes

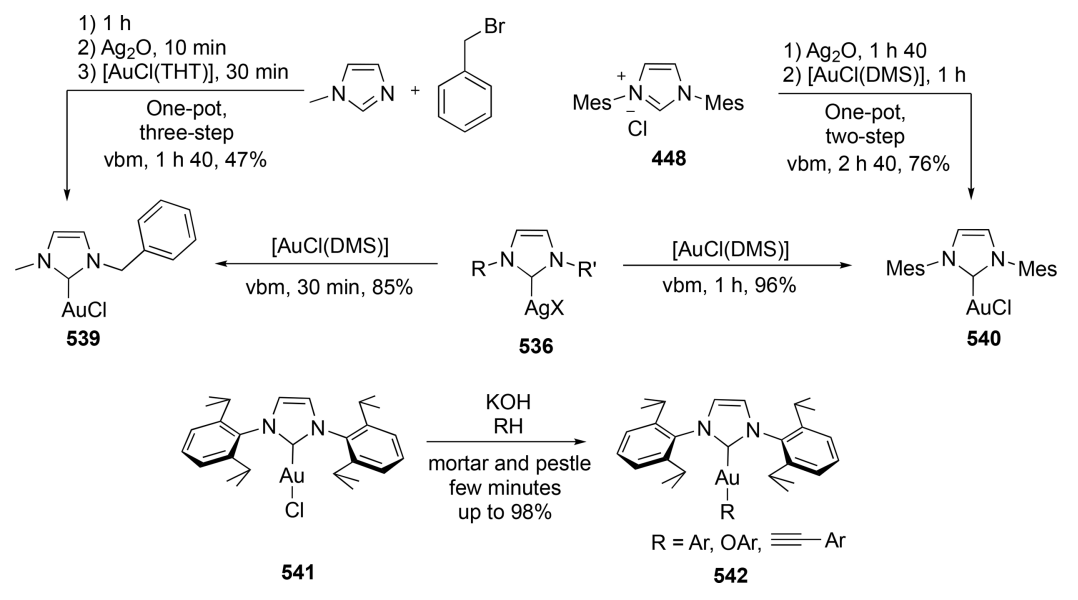

challenging silver complexes featuring sterically hindered ligands. As for copper complexes, this methodology facilitated access to homoleptic silver complexes, which were rarely described because of synthetic issues (Scheme 162, reaction C). ${ }^{315}$ Starting from imidazolium salts featuring a noncoordinating anion $\left(\mathrm{BF}_{4}, \mathrm{PF}_{6}\right)$, complexes 537 were prepared in yields of up to $94 \%$ and within a short time $(1-3 \mathrm{~h})$. Due to the relatively low reactivity of imidazolium tetrafluoroborate and hexafluorophosphate salts, sodium hydroxide was added into the jar to perform the metalation.

Apart from their use in catalysis, gold complexes are wellknown in pharmaceutical science for their applications as drugs to treat rheumatoid arthritis. Moreover, $\mathrm{Au}(\mathrm{I})$ phosphine complexes display a wide spectrum of anticancer activity in vivo. ${ }^{7}$ Interestingly, $\mathrm{Au}(\mathrm{III})$ is isoelectronic and isostructural of $\mathrm{Pt}(\mathrm{II})$. Therefore, $\mathrm{Au}$ (III) analogues of $\mathrm{Pt}(\mathrm{II})$ complexes were investigated for their biological properties. ${ }^{142}$

As for NHC palladium and copper complexes (Scheme 131 and Scheme 160), the transmetalation of $[\mathrm{AgCl}(\mathrm{NHC})]$ into $[\mathrm{AuCl}(\mathrm{NHC})]$ in a vibratory ball-mill Retsch MM200 operated at $25 \mathrm{~Hz}$ was found to be highly efficient (Scheme 163). ${ }^{314}$ Complex 539 was also obtained from $N$-methylimidazole in a one-pot, three-step procedure, which gave the desired product in $47 \%$ yield ( $78 \%$ average yield on each step) in only $100 \mathrm{~min}$ compared to hours in solution. The same procedure was used to form $\mathrm{N}, \mathrm{N}$-diaryl NHC gold complex 540 in high yield. ${ }^{262}$ In 2013, the Nolan group described the one-pot mechanotransformation of $[\mathrm{AuCl}(\mathrm{IPr})] \mathbf{5 4 1}$ into [Au(IPr)R $\mathbf{5 4 2}$ by grinding it with a mortar and pestle with an excess of $\mathrm{KOH}$. [Au(IPr) OH], a complex which was previously synthesized in solution, was formed intermediately. Ulterior grinding with an organic $\mathrm{R}-\mathrm{H}$ substrate in stoichiometric amounts produced complexes 542 (Scheme 163). ${ }^{316}$ The overall synthesis was complete within a few minutes in a mortar even for highly challenging substrates, whereas hours were necessary in solution (time is dependent on the $\mathrm{p} K_{\mathrm{a}}$ of the organic substrate). Surprisingly, more acidic phenols and carboxylic acids did not react in the solid-state synthesis, although they were the most reactive in solution.

In their publication on the mechanochemical oxidation of zerovalent metals, as well as for palladium oxidation (Scheme 134), the Frišcić group demonstrated the mechanochemical oxidation of metallic gold to form $\left[\mathrm{AuCl}\left(\mathrm{PPh}_{3}\right)\right] 544$ in a twostep procedure (Scheme 164). ${ }^{317}$ The use of ball milling enables the avoidance of strong acids or molten salts as oxidants.

Scheme 164. Mechanochemical Oxidation of Metallic Gold

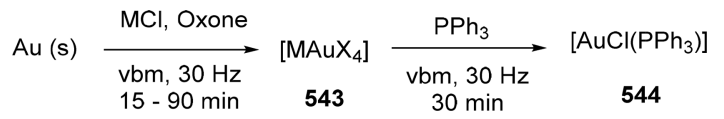

In a one-pot, two-step synthesis in a ball mill, dinuclear gold complexes 546 ( $\left.\left[\mathrm{Au}_{\mathbf{2}} \text { (dpephos) }\right)_{2}\right] \mathrm{X}_{2}, \mathrm{X}=\mathrm{NO}_{3}, \mathrm{CF}_{3} \mathrm{SO}_{3}, \mathrm{BF}_{4}$, $\mathrm{PF}_{6}$, and $\mathrm{SbF}_{6}$, dpephos = bis[(2-diphenylphosphino)phenyl $]$ ether) were formed in only $10 \mathrm{~min}$ of grinding (Scheme 165). ${ }^{318}$ Interestingly, the compounds display aurophilicity-

Scheme 165. Dichloromethane-Assisted Mechanochemical Synthesis of $\left[\mathrm{Au}_{2}(\mathrm{dpephos})_{2}\right] \mathrm{X}_{2}$
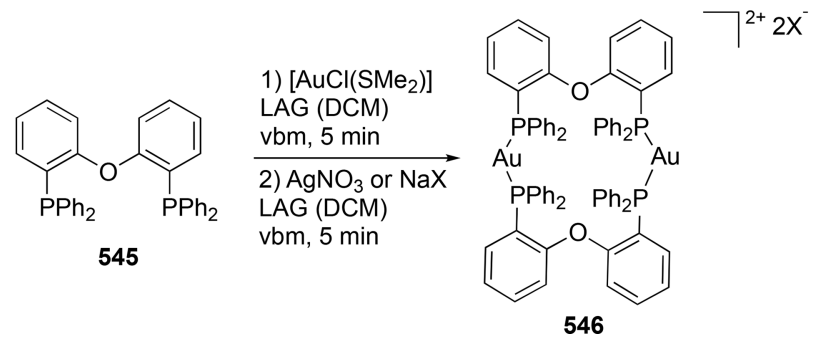

triggered multicolor emission. In the solid state, the emission wavelength of the compounds is dependent on the working temperature, the excitation wavelength, and pressure. Moreover, reversible mechanochromic luminescence was demonstrated upon exposure of the compounds to solvent vapors during grinding.

11.2. Microwave Synthesis of Group 11 Metal Complexes Copper(II) complexes featuring Schiff base ligands were intensely studied in recent years due to their biological properties as antimicrobial or antioxidizing agents. In 2012, Ramakanth et al. reported the synthesis of a $\left[\mathbf{C u}(79)_{2}\right]$ complex (Table 8, entry 1). ${ }^{133}$ Ligand 79 was added to copper(II) acetate monohydrate in ethanol, in a 2:1 ratio. The reaction mixture was then heated in a microwave oven at 600 $\mathrm{W}$ for $18 \mathrm{~s}$ to obtain the product in $92 \%$ yield. This represents a drastic time reduction since $5.5 \mathrm{~h}$ was necessary to obtain the same complex in $81 \%$ yield under conventional reflux conditions. 
Table 8. Microwave Synthesis of $\mathrm{Cu}(\mathrm{II})-$ Schiff Base Complexes versus Conventional Heating ${ }^{a}$

\begin{tabular}{|c|c|c|c|c|c|c|}
\hline \multirow[b]{2}{*}{ entry } & \multirow[b]{2}{*}{ copper complex } & \multicolumn{2}{|c|}{ microwave synthesis } & \multicolumn{2}{|c|}{ conventional heating } & \multirow[b]{2}{*}{ ref } \\
\hline & & time & yield (\%) & time $(\mathrm{h})$ & yield (\%) & \\
\hline 1 & {$\left[\mathrm{Cu}(79)_{2}\right]$} & $18 \mathrm{~s}$ & 92 & 5.5 & 81 & 133 \\
\hline 2 & {$\left[\mathrm{Cu}(74)\left(\mathrm{H}_{2} \mathrm{O}\right)\right]$} & $7 \mathrm{~min}$ & 85 & 7 & 62 & 95 \\
\hline 3 & {$[\mathrm{Cu}(75) \mathrm{Cl}] \cdot \mathrm{H}_{2} \mathrm{O}$} & $8 \mathrm{~min}$ & 83 & 7 & 66 & 95 \\
\hline 4 & {$\left[\mathrm{Cu}\left(76_{2} \mathrm{Cl}_{2}\right] \cdot 2 \mathrm{H}_{2} \mathrm{O}\right.$} & $6 \mathrm{~min}$ & 84 & 6 & 64 & 235 \\
\hline 5 & {$\left[\mathrm{Cu}(77)_{2} \mathrm{Cl}_{2}\right] \cdot 2 \mathrm{H}_{2} \mathrm{O}$} & $7 \mathrm{~min}$ & 82 & 7 & 68 & 235 \\
\hline 6 & {$[\mathrm{Cu}(88)] \cdot 2 \mathrm{H}_{2} \mathrm{O}$} & $6 \mathrm{~min}$ & 85 & 7 & 66 & 98 \\
\hline 7 & {$\left[\mathrm{Cu}(78)_{2}\right] \cdot 2 \mathrm{H}_{2} \mathrm{O}$} & $7 \mathrm{~min}$ & 82 & 7 & 65 & 96 \\
\hline 8 & {$\left[\mathrm{Cu}(80)_{2}\right]$} & $8 \min$ & 84 & 6 & 63 & 96 \\
\hline 9 & {$\left[\mathrm{Cu}(69)_{2}\left(\mathrm{H}_{2} \mathrm{O}\right)_{2}\right] \mathrm{Cl}_{2}$} & $8 \mathrm{~min}$ & 83 & 7 & 60 & 272 \\
\hline 10 & {$\left[\mathrm{Cu}(70)_{2}\right]$} & $7 \mathrm{~min}$ & 85 & 6 & 67 & 272 \\
\hline 11 & {$\left[\mathrm{Cu}(81)_{2}\right]$} & $7 \mathrm{~min}$ & 86 & 6 & 74 & 99 \\
\hline 12 & {$\left[\mathrm{Cu}(82)_{2}\right] \cdot \mathrm{H}_{2} \mathrm{O}$} & $7 \mathrm{~min}$ & 81 & 8 & 71 & 99 \\
\hline 13 & {$\left[\mathrm{Cu}(84)_{2}\right] \cdot 2 \mathrm{H}_{2} \mathrm{O}$} & $8 \mathrm{~min}$ & 82 & 7 & 69 & 97 \\
\hline 14 & {$\left[\mathrm{Cu}(83)\left(\mathrm{H}_{2} \mathrm{O}\right)\right] \mathrm{Cl}$} & $7 \mathrm{~min}$ & 84 & 7 & 68 & 97 \\
\hline 15 & {$\left[\mathrm{Cu}(85)_{2}\right] \cdot 2 \mathrm{H}_{2} \mathrm{O}$} & $8 \mathrm{~min}$ & 84 & 8 & 64 & 100 \\
\hline 16 & {$\left[\mathrm{Cu}(86)_{2}\left(\mathrm{H}_{2} \mathrm{O}\right)_{2}\right]$} & $7 \mathrm{~min}$ & 82 & 8 & 68 & 100 \\
\hline 17 & {$[\mathrm{Cu}(71)] \cdot \mathrm{H}_{2} \mathrm{O}$} & $8 \mathrm{~min}$ & 84 & 10 & 55 & 134 \\
\hline 18 & {$[\mathrm{Cu}(89)]$} & $8-10 \mathrm{~min}$ & $74-80$ & hours & 55 & 236 \\
\hline 19 & {$\left[\mathrm{Cu}(103)_{2}\right]$} & $1 \mathrm{~h}$ & 83 & 0.5 & 76 & 273 \\
\hline 20 & {$[\mathrm{Cu}(104) \mathrm{Cl}]$} & $1.5 \mathrm{~h}$ & 77 & 2 & 62 & 273 \\
\hline 21 & {$[\mathrm{Cu}(105) \mathrm{Cl}]$} & $2.5 \mathrm{~h}$ & 68 & 3 & 54 & 273 \\
\hline 22 & {$\left[\mathrm{Cu}(106)_{2}\right]$} & $2.1 \mathrm{~h}$ & 73 & 3.5 & 65 & 273 \\
\hline
\end{tabular}

Between 2012 and 2016, the Jain group reported the microwave synthesis of numerous copper(II) complexes with Schiff bases as ligands. Syntheses were performed in an open glass vessel using a modified microwave oven model $2001 \mathrm{ETB}$ with a rotating tray and a microwave energy output of $800 \mathrm{~W}$. Microwave reactions were performed using on/off cycling to control the temperature. $\left[\mathbf{C u}(\mathbf{7 4})\left(\mathbf{H}_{\mathbf{2}} \mathbf{O}\right)\right],{ }^{95}[\mathbf{C u}(\mathbf{7 5}) \mathbf{C l}]$. $\mathbf{2 H}_{\mathbf{2}} \mathbf{O},^{95}\left[\mathrm{Cu}(\mathbf{7 6})_{2} \mathbf{C l}_{\mathbf{2}}\right] \cdot \mathbf{2 H}_{\mathbf{2}} \mathbf{O},^{235}\left[\mathrm{Cu}(\mathbf{7 7})_{2} \mathrm{Cl}_{\mathbf{2}}\right] \cdot \mathbf{2} \mathbf{H}_{\mathbf{2}} \mathbf{O},{ }^{235}$ $[\mathbf{C u}(\mathbf{8 8})] \cdot \mathbf{2 H}_{\mathbf{2}} \mathbf{\mathrm { O }},{ }^{98}\left[\mathbf{C u}(\mathbf{7 8})_{2}\right] \cdot \mathbf{2 H}_{\mathbf{2}} \mathbf{O},{ }^{96}\left[\mathbf{C u}(\mathbf{8 0})_{2}\right],{ }^{96}[\mathbf{C u}-$ $\left.(69)_{2}\left(\mathrm{H}_{2} \mathrm{O}\right)_{2}\right] \mathrm{Cl}_{2},{ }^{272}\left[\mathrm{Cu}(70)_{2}\right],{ }^{272}\left[\mathrm{Cu}(\mathbf{8 1})_{2}\right],{ }^{99}\left[\mathrm{Cu}(\mathbf{8 2})_{2}\right] \cdot$ $\mathbf{2 H}_{2} \mathbf{O},{ }^{99}\left[\mathbf{C u}(\mathbf{8 4})_{2}\right] \cdot \mathbf{2 H}_{2} \mathbf{O},{ }^{97}\left[\mathbf{C u}(\mathbf{8 3})\left(\mathbf{H}_{2} \mathbf{O}\right)\right] \mathbf{C l},{ }^{97}[\mathbf{C u}-$ $\left.(\mathbf{8 5})_{2}\right] \cdot 2 \mathbf{H}_{2} \mathbf{O},^{100}$ and $\left[\mathbf{C u}(\mathbf{8 6})_{2}\left(\mathbf{H}_{2} \mathbf{O}\right)_{2}\right]^{100}$ (Table 8, entries 2 to 16 , see Figure 1 for ligand structures) were synthesized using a similar procedure. Before microwave irradiation, the ligand and $\left[\mathrm{CuCl}_{2}\right] \cdot \mathbf{2} \mathrm{H}_{2} \mathrm{O}$ were ground in a stoichiometric ratio of $1: 1$ or $2: 1$. After 6 to $8 \mathrm{~min}$ of irradiation in $3-5 \mathrm{~mL}$ of dry ethanol, the corresponding complexes were recovered in yields between 81 and $86 \%$. Microwave irradiation outperformed classical conditions, enabling the reaction time to be reduced from hours to only minutes and to increase the yield from $60-70 \%$ to more than $80 \%$.

In 2014 , the microwave synthesis of $[\mathbf{C u}(7 \mathbf{1})] \cdot \mathrm{H}_{2} \mathrm{O}$ was reported. ${ }^{134}$ Only $8 \mathrm{~min}$ of microwave irradiation was necessary to isolate the complex in $84 \%$ yield; in comparison, conventional heating required $10 \mathrm{~h}$ to obtain a moderate $55 \%$ yield (Table 8, entry 17). In DMF, $\left[\mathbf{C u}(\mathbf{1 0 3})_{2}\right],[\mathbf{C u}(\mathbf{1 0 4}) \mathbf{C l}]$, $[\mathbf{C u}(\mathbf{1 0 5}) \mathbf{C l}]$, and $\left[\mathbf{C u}(\mathbf{1 0 6})_{2}\right]$ were synthesized under microwave irradiation. The reaction times were slightly reduced, and the yields were improved compared to conventional syntheses. ${ }^{272}$

Once again (see cobalt and nickel luminol complexes, Scheme 112), Aswathy et al. showed that coordination of a luminol derivative onto copper was detrimental to the fluorescence lifetime (Scheme 166). Nevertheless, copper(II) complex 547 could be obtained in 5 min irradiation and in $80 \%$ yield starting from $\mathrm{CuCl}_{2}{ }^{237}$

Scheme 166. Microwave-Assisted Synthesis of a Copper Complex Featuring a Luminol Derivative Ligand
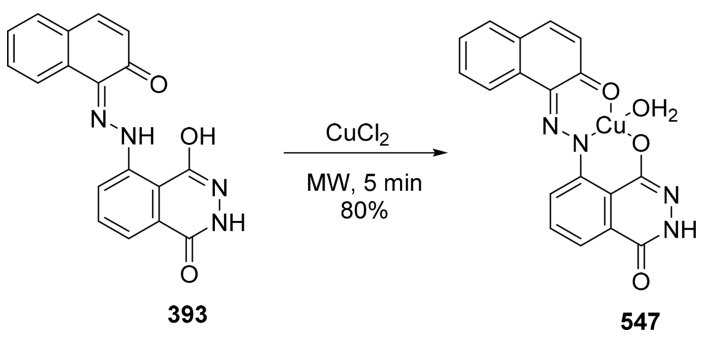

Macrocyclic copper(II) complexes $\mathbf{5 4 8}$ could also be obtained through microwave activation (Scheme 167 and

Scheme 167. Microwave Synthesis of Macrocyclic Cu(II) Complexes
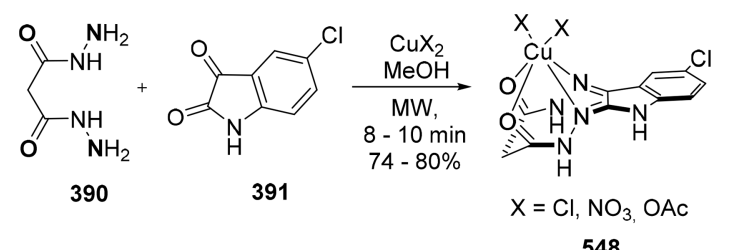

548

Table 8, entry 18). ${ }^{236}$ Malonic acid dihydrazide 390, 5chloroisatin 391, and $\mathrm{CuX}_{2}\left(\mathrm{X}=\mathrm{Cl}, \mathrm{NO}_{3}, \mathrm{OAc}\right)$ were mixed in a grinder in a 1:1:1 ratio. The Schiff base ligand was formed in situ. The reaction mixture was then irradiated in an UWave1000 Sineo microwave synthesizer for $8-10 \mathrm{~min}$ to obtain the corresponding copper complexes in yields between 74 and 
$80 \%$. The reaction time was considerably reduced from hours to minutes, and yields were increased, compared with the 55\% obtained under reflux.

The conventional synthesis of the $\mathrm{Cu}$ (II) phthalocyanine complex takes several hours under reflux; therefore, a more sustainable synthesis was developed using microwave irradiation (Scheme 168). ${ }^{319}$ Solvent-free synthesis was performed

Scheme 168. Synthesis of a Cu(II) Phthalocyanine Complex

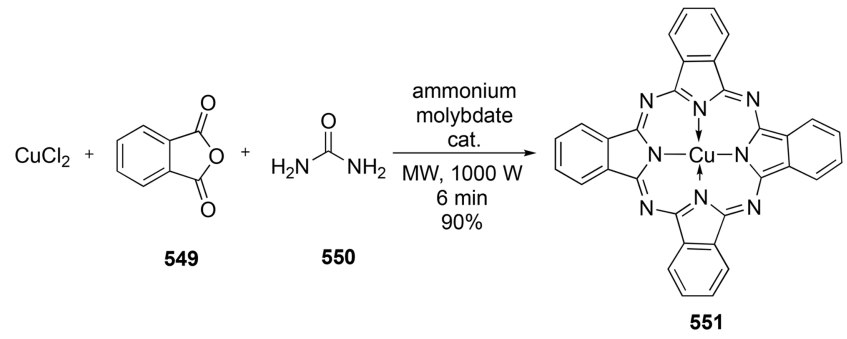

by first grinding anhydrous $\mathrm{CuCl}_{2}$, phthalic anhydride 549 in a 1:4 ratio, urea $\mathbf{5 5 0}$ in large excess, and ammonium molybdate in catalytic quantity with a mortar and pestle. The homogenized reaction mixture was then transferred to a reactor and placed in a laboratory microwave oven $(1000 \mathrm{~W})$ for 6 min ( 6 cycles of $1 \mathrm{~min}$ of heating with pauses of $5 \mathrm{~s}$ ) to isolate desired product $\mathbf{5 5 1}$ in $\mathbf{9 0 \%}$ yield.

Microwave irradiation also enabled the drastic acceleration of NHC copper, silver, and gold complex synthesis (Scheme 169). ${ }^{320}$ Using a CEM Discover unit at $110{ }^{\circ} \mathrm{C}$ with

Scheme 169. Microwave Formation of NHC Copper and Silver Complexes

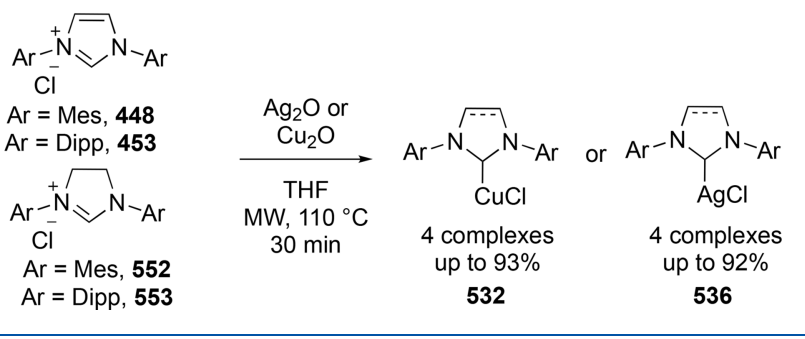

simultaneous cooling, imidazolium salts (IMes $\cdot \mathrm{HCl} 448=$ 1,3-bis(2,4,6-trimethylphenyl)- $1 \mathrm{H}$-imidazolium chloride, SIMes $\cdot \mathrm{HCl} \mathbf{5 5 2}=$ 1,3-bis(2,4,6-trimethylphenyl)- $1 \mathrm{H}$-imidazolinium chloride, $\mathrm{IPr} \cdot \mathrm{HCl} 453$ = 1,3-bis(2,6-diisopropylphenyl)$1 \mathrm{H}$-imidazolium chloride, and $\mathrm{SIPr} \cdot \mathrm{HCl} \mathbf{5 5 3}=1,3$-bis $(2,6$ diisopropylphenyl)- $1 H$-imidazolinium chloride) were reacted with copper(I) oxide for $30 \mathrm{~min}$ to produce the corresponding $[\mathbf{C u C l}(\mathrm{NHC})]$ complexes $\mathbf{5 3 2}$ in yields between 73 and $98 \%$. Importantly, reactions required $24 \mathrm{~h}$ when using classical refluxing conditions. Interestingly, yields were better for more hindered salts, 453 and 553. The synthesis was scaled up to $2 \mathrm{~g}$ of $[\mathrm{CuCl}(453)]$ in less than $1 \mathrm{~h}$ (93\% yield). The same reaction improvement was observed for the formation of four [AgCl(NHC)] complexes 536 from silver(I) oxide.

The synthesis of gold complexes was found to be more challenging since direct metalation was only efficient with IPr.

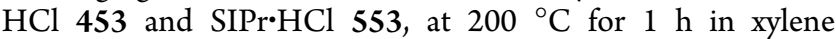
(Scheme 170, metalation). When this procedure was attempted for the synthesis of $[\mathrm{AuCl}(\mathrm{IMes})] \quad \mathbf{5 5 8}$ and $[\mathrm{AuCl}(\mathrm{SIMes})] 559$, a mixture of products was obtained. To avoid this, transmetalation of corresponding copper(I) complexes 556-557 was performed, producing the desired gold complexes in yields of up to $92 \%$ after $5 \mathrm{~min}$ at $65{ }^{\circ} \mathrm{C}$ (Scheme 170, transmetalation).

Transmetalation of gold complexes with arylboronic acids was also investigated using microwave activation since aryl gold complexes are highly valuable molecules in numerous applications (emissive materials, metallodrugs, mechanistic intermediates). ${ }^{321}$ Reacting [AuCl(JohnPhos)] 560 (JohnPhos $=\operatorname{di}($ tert-butyl $)\left(1,1^{\prime}\right.$-biphenyl-2-yl $)$-phosphine $)$ or $[\operatorname{AuCl}(t \mathrm{BuXPhos})] \mathbf{5 6 1}\left(t \mathrm{BuXPhos}=\right.$ di-tert-butyl $\left(2^{\prime}, 4^{\prime}, 6^{\prime}\right.$ triisopropylbiphenyl-2-yl)phosphine), $\mathrm{ArB}(\mathrm{OH})_{2}$, and $\mathrm{Cs}_{2} \mathrm{CO}_{3}$ in THF in a $1: 2: 2$ ratio at $70{ }^{\circ} \mathrm{C}$ for $30-90 \mathrm{~min}$ under microwave activation proved highly efficient (Scheme 171). In

\section{Scheme 171. Synthesis of Aryl Gold(I) Species by} Microwave Irradiation

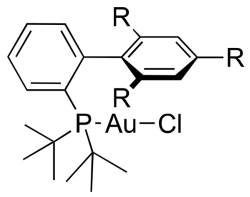

$\mathrm{R}=\mathrm{H}, \mathbf{5 6 0}$

$\mathrm{R}=\operatorname{Pr}, \mathbf{5 6 1}$

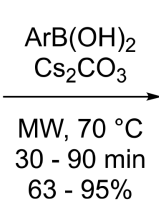

$63-95 \%$

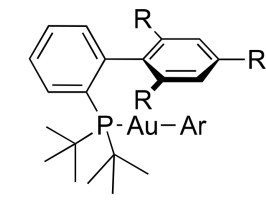

562 addition to improvements in terms of reaction time (up to $60 \mathrm{~h}$ under reflux), the use of microwaves enabled reduction in arene formation and working with electron-rich, electron-poor, or even ortho-substituted boronic acids. Notably, benzothiophene-2-boronic acid, which is prone to protodeboronation, could be transmetalated efficiently using this microwave methodology.

Gold complexes featuring nitrogen-containing ligands could also be obtained with enhanced results through microwave activation. An early paper reported the first microwave synthesis of the $\left[\mathbf{A u C l}(\right.$ terpy) $] \mathbf{C l}_{\mathbf{2}} \cdot \mathbf{3} \mathbf{H}_{\mathbf{2}} \mathbf{O}$ complex. ${ }^{91}$ Using a sealed Teflon reactor in a microwave oven with a power level of 500-600 W for $1 \mathrm{~min}$, the desired complex was synthesized, from $\mathrm{HAuCl}_{4}$ and terpy, in $37 \%$ yield. The formation of small quantities of the $[\mathrm{Au}(\text { terpy }) \mathbf{C l}]_{2}\left[\mathbf{A u C l}_{2}\right]_{3}\left[\mathbf{A u C l}_{4}\right]$ intermediate was also observed. Using conventional reflux conditions, the reaction required $24 \mathrm{~h}$ to obtain the desired complex.

Scheme 170. Microwave Syntheses of NHC Gold Complexes

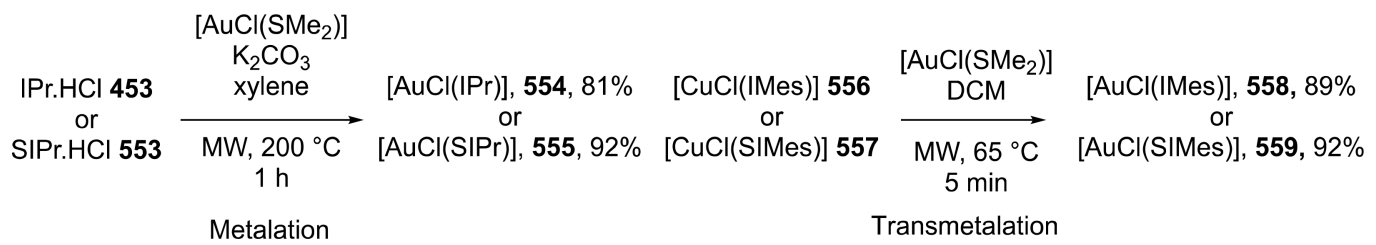


Scheme 172. Syntheses of Pyridine-Containing Gold Complexes
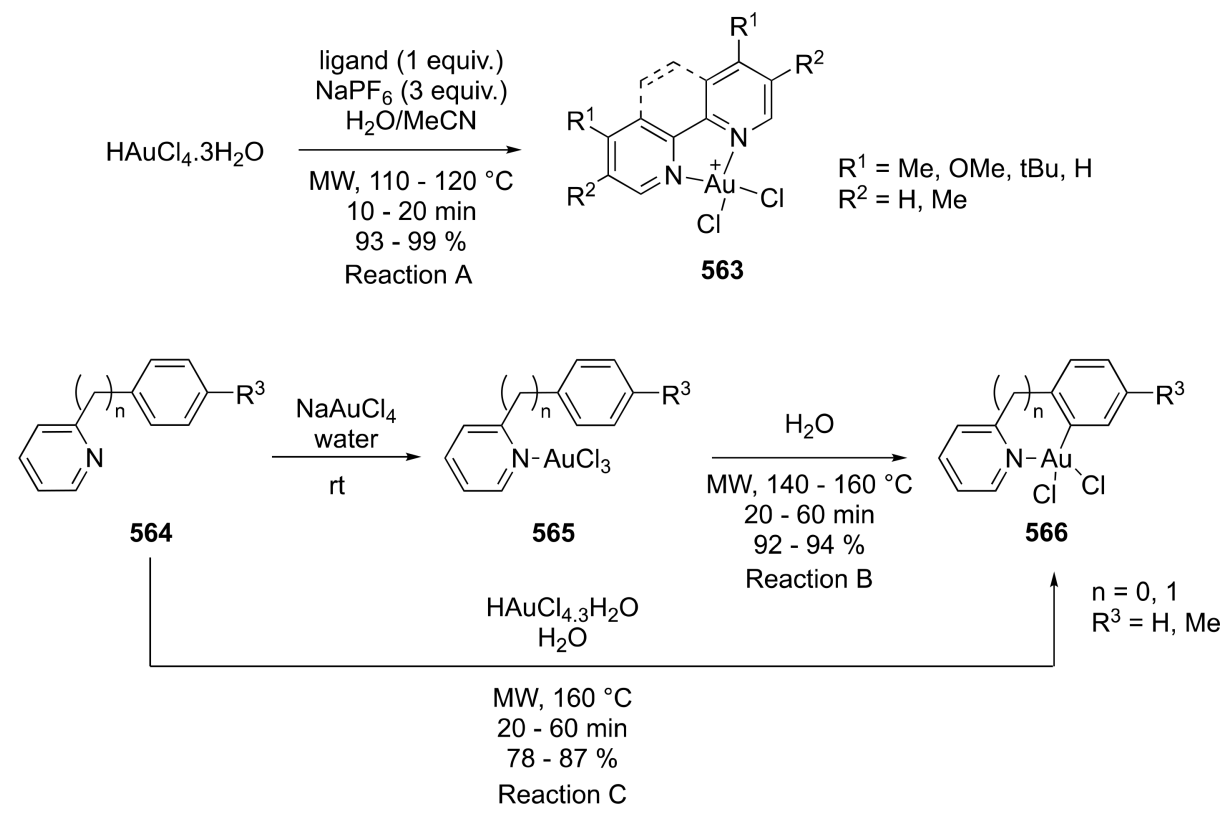

Coordination of bipyridine-type ligands starting from $\mathrm{HAuCl}_{4} \cdot 3 \mathrm{H}_{2} \mathrm{O}$ was also reported using microwave irradiation. ${ }^{322}$ Ligands, $\mathrm{HAuCl}_{4} \cdot 3 \mathrm{H}_{2} \mathrm{O}$, and $\mathrm{NaPF}_{6}$ in a $1: 1: 3$ ratio were heated at $120{ }^{\circ} \mathrm{C}$ in a sealed Teflon vessel using a Milestone MicroSYNTh oven to produce pure $\left[\mathrm{AuCl}_{\mathbf{2}}(\mathbf{N}-\right.$ $\mathbf{N})]\left[\mathrm{PF}_{6}\right] 563$ in $10-30 \mathrm{~min}$ and excellent yields (Scheme 172 , reaction A). In addition to a reduced reaction time compared to the classical synthesis, the microwave methodology allowed for an otherwise necessary recrystallization step to be avoided. Access to $\left[\mathrm{AuCl}_{2}(\mathrm{C}-\mathrm{N})\right]$ complexes $566(\mathrm{C}-$ $\mathrm{N}=$ cyclometalated 2-phenylpyridine 2-benzylpyridine-type ligands) was possible either from pyridine-ligated gold complex 565 through $\mathrm{C}-\mathrm{H}$ activation (Scheme 172 , reaction $\mathrm{B}$ ) or directly from the $\mathrm{C}-\mathrm{N}$ ligand 564 and $\mathrm{HAuCl}_{4} \cdot 3 \mathrm{H}_{2} \mathrm{O}$ in a onepot approach (Scheme 172, reaction C). Using both pathways, yields and reaction times were dramatically improved in comparison with refluxing conditions. The only limitation was the unsuccessful cyclometalation with 2-ethylpyridine.

11.3. Ultrasounds for the Synthesis of Copper and Silver Complexes

Sonochemical syntheses of copper and silver complexes are quite rare in the literature, and to the best of our knowledge, no example has been reported for gold. In 2015, the Morsali group described the ultrasonic synthesis of $[\mathrm{Cu}(567)]$. $(\mathrm{EtOH})_{0.5} \cdot\left(\mathrm{H}_{2} \mathrm{O}\right)_{1.5}$ and $\left[\mathrm{Cu}(568)_{2}\right]$ at $40 \mathrm{kHz}$ for $1 \mathrm{~h}$ (Figure 10). ${ }^{323}$ Interestingly, the same conditions under reflux were also efficient in yielding the expected complexes, but the powder obtained was not nanosized. Regarding silver, the only application of sonochemistry dealt with anion metathesis starting from $\left[\operatorname{Ag}(569)_{2}\right] \mathrm{Cl}$ to yield $\left[\operatorname{Ag}(569)_{2}\right] \mathrm{BF}_{4}$, a

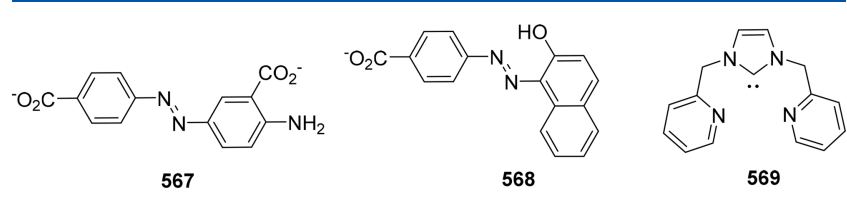

Figure 10. Ligands used for ultrasonic synthesis of copper and silver complexes. photoluminescent complex. ${ }^{324}$ Only 5 min was required to afford the classical homoleptic tetrafluoroborate complex (89\% yield) using an ultrasonic cleaner, which could be transformed, upon addition of $\mathrm{AgBF}_{4}$, into a trimetallic species featuring different luminescent properties.

\subsection{Continuous Flow for the Synthesis of NHC Copper} Complexes

As discussed above, continuous flow is still an emerging technology, particularly in relation to organometallic synthesis. To date, only copper complexes have been synthesized using this enabling technology. In 2013, the McQuade group described the first continuous synthesis of $[\mathrm{CuCl}(\mathrm{NHC})]$ complexes 532 by flowing a DCM/toluene solution of imidazolium salts 169 through a packed bed of solid $\mathrm{Cu}_{2} \mathrm{O}$ suspended in molecular sieves to prevent leaching of finegrained $\mathrm{Cu}_{2} \mathrm{O}$ and to remove water produced during the reaction. ${ }^{325}$ The use of solids in flow is generally avoided since they could cause pump or reactor clogging. Using a packed bed of $\mathrm{Cu}_{2} \mathrm{O}$ downstream of the pumps, however, allowed the formation of the desired complexes and increased the rate of complex synthesis due to the increasing interaction between solid $\mathrm{Cu}_{2} \mathrm{O}$ and the imidazolium salt. When imidazolium triflate and tetrafluoroborate were used instead of imidazolium chloride, the reaction did not afford the desired complexes. Since some imidazolium salts are not isolatable as their chloride salt, an ion exchange column was used upstream to form an intermediate of the chloride salt that would then react with $\mathrm{Cu}_{2} \mathrm{O}$ (Scheme 173). These examples show that using $\mathrm{Cu}_{2} \mathrm{O}$ for the synthesis of $\mathrm{NHC}-$ copper complexes is limited when considering halide copper(I) complex production. Despite this, the formation of complexes that were impossible to make in classical solution was enabled following this method. For example, 1,3-dicyclohexylimidazolidinium chloride reacted efficiently in flow, whereas the corresponding urea was obtained in batch conditions. To demonstrate that this flow methodology was scalable, $1 \mathrm{~g}$ of $[\mathrm{CuCl}(\mathrm{SIMes})]$ was produced in $16 \mathrm{~min}$.

In 2015, Chapman et al. reported the electrochemical continuous flow synthesis of NHC copper(I) complexes 532 
Scheme 173. Schematic Representation of the NHC Cu Synthesis Reactor in Continuous Flow Using $\mathrm{Cu}_{2} \mathrm{O}$

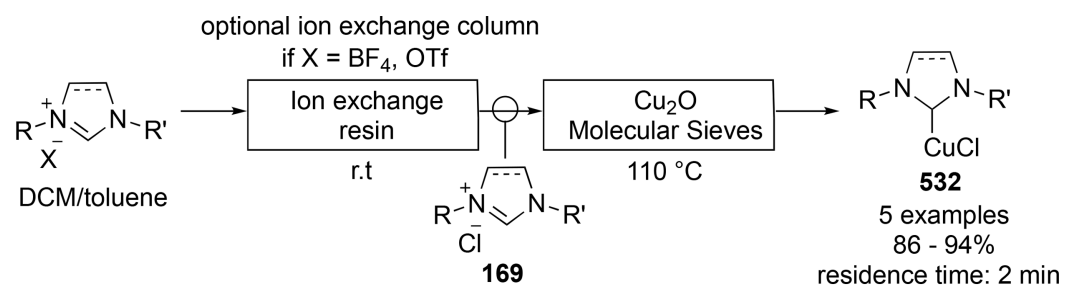

(Figure 11). ${ }^{326}$ During the electrochemical reaction, imidazolium ions were reduced at the cathode, and oxidation of the

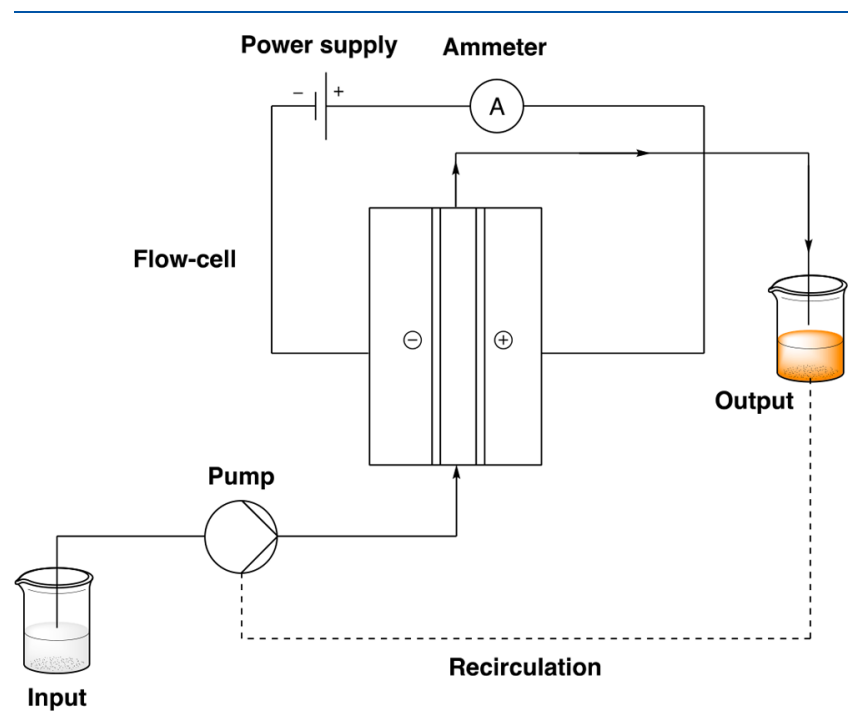

Figure 11. Schematic diagram of the electrochemical synthesis of NHC Cu complexes.

sacrificial copper anode liberates $\mathrm{Cu}^{+}$ions into the solution. The most efficient homemade reactor was prepared in the following manner: six square copper plates, alternating between the anode and cathode and separated by PTFE spacers, were assembled. This design allowed the optimization of all of the reactor parameters. Six copper complexes were synthesized in high yields (91-95\%) within a short time (7$30 \mathrm{~min}$ ). Time was increased to $300 \mathrm{~min}$ for the synthesis of one of these complexes in recirculation mode. The method was quite general since different counteranions $\left(\mathrm{Cl}, \mathrm{Br}, \mathrm{PF}_{6}\right)$, bulky and base-sensitive $\mathrm{N}$-substituents, as well as a macrocyclic imidazolium salts could be used.

\section{GROUP 12}

\subsection{Mechanosynthesis of Group 12 Metal Complexes}

The Friščic group described a one-pot associative synthesis of zinc complexes that combined solid-state metal oxidation and direct coordination of the ligand. Grinding $\mathrm{Zn}$ powder with 3,5-di-tert-butyl ortho-quinone 151 in the absence of solvent gave poor conversions. In contrast, liquid-assisted grinding of the same mixture in the presence of a 1:1 mixture of toluene and water resulted in the corresponding discrete complexes in 90 min of reaction (Scheme 174). ${ }^{124}$ Interestingly, grinding $\mathrm{Zn}$ powder with 1,4-bis(2-(pyridin-4-yl)vinyl)benzene $\mathbf{5 7 0}$ and ortho-quinone 151 led to the formation of two different species depending on the toluene:water proportion. In a 1:1 ratio, formation of the dinuclear $\left[\mathbf{Z n}_{2}(\mathbf{S Q})_{4}(\mathbf{5 7 0})\right]$ complex 576 was observed, whereas in a 4:1 ratio, coordination polymer
Scheme 174. Mechanochemical Reaction of ortho-Quinone with Zn Powder ${ }^{a}$

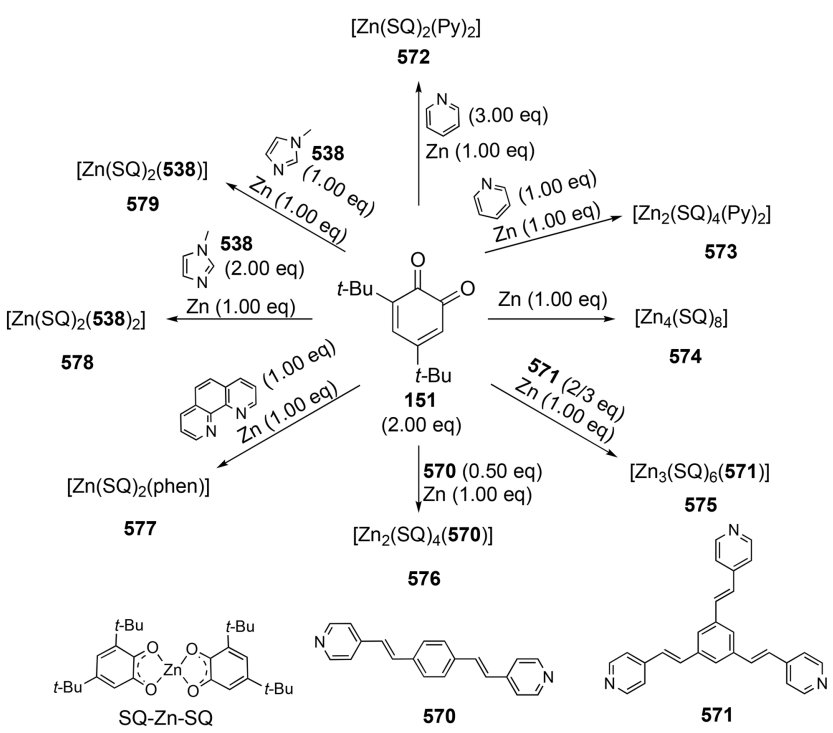

${ }^{a}$ Reaction conditions: vbm, $25 \mathrm{~Hz}\left(60 \mu \mathrm{L}, \mathrm{PhMe} / \mathrm{H}_{2} \mathrm{O}\right.$ 1:1 ratio), 90 $\min , \mathrm{NMI}=N$-methylimidazole.

$\left[\mathrm{Zn}(\mathrm{SQ})_{2}(\mathbf{5 7 0})\right]$ was obtained. Notably, the trinuclear zinc complex 575 was also formed using the tripodal 1,3,5-tris(2(pyridin-4-yl)vinyl)benzene ligand 571. XRD data revealed that trigonal complexes arrange into a loosely packed structure with solvent molecules occupying vacant cavities. Interestingly, all syntheses could also be performed using $\mathrm{ZnO}$ as a metal source starting from a 1:1 mixture of ortho-quinone 151 and the corresponding catechol. The conditions were identical to those with $\mathrm{Zn}$ powder.

The James group, in their report dealing with the formation of salen complexes of nickel (Scheme 139) and copper (Scheme 158), also described the isolation of zinc-salen complex 580. ${ }^{270}$ The mechanosynthesis could be performed either through a one-pot, two-step sequence or all at once by milling salicylaldehyde, ethylene diamine, and zinc oxide at 25 $\mathrm{Hz}$ for $1 \mathrm{~h}$ (Scheme 175). Interestingly, addition of methanol as a liquid assistant was necessary in the sequential approach but not in the multicomponent approach.

The same group demonstrated that the synthesis of $\mathrm{Zn}-$ salen complexes was possible using twin-screw extrusion. ${ }^{327}$ Starting from the appropriate aldehyde $\mathbf{5 8 1}$ and diamine $\mathbf{5 8 2}$ in the presence of $\operatorname{zinc}(\mathrm{II})$ acetate, the multicomponent reaction yielded corresponding complex $\mathbf{5 8 3}$ with full conversion (Scheme 176). It is important to note that the formation of the bisimine ligand could not be performed efficiently without the addition of the metal source and thus that a sequential synthesis of the complex was not possible. 
Scheme 175. Mechanosynthesis of a $\mathrm{Zn}-$ Salen Complex

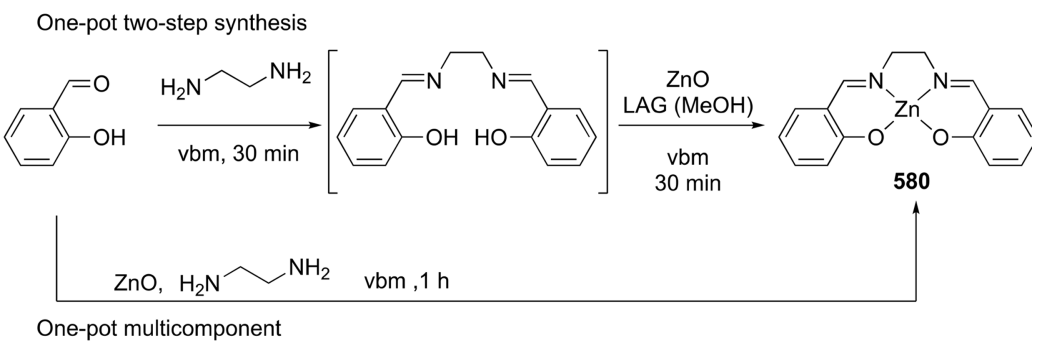

Scheme 176. Multicomponent Preparation of a $\mathrm{Zn}-$ Salen Complex

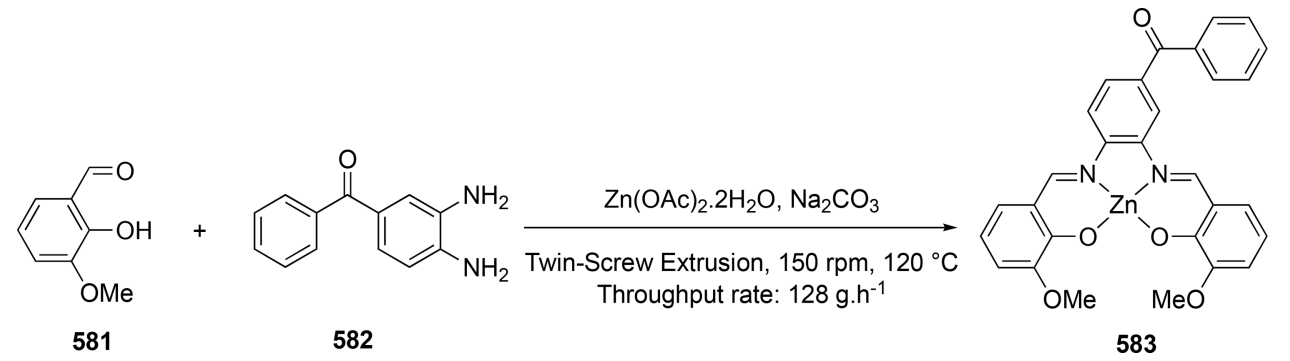

The use of twin-screw extrusion allowed a scale-up of the synthesis, reaching a throughput rate of $128 \mathrm{~g} \cdot \mathrm{h}^{-1}$.

As already described with a copper source (Figure 9), manual grinding of anhydrous gabapentin 584 with $\mathrm{ZnCl}_{2}$ for 5 min with an agate mortar and pestle gave access to $\left.\left[\mathrm{ZnCl}_{2} \text { (gabapentin) }\right)_{2}\right] \mathbf{5 8 5}$ in quantitative yield (Scheme 177 , reaction A). ${ }^{301}$ Similarly, $\left[\mathbf{M C l}_{2}(\mathbf{C E A P})_{2}\right] \mathbf{5 8 6}$ and the

\section{Scheme 177. Mechanosynthesis of Gabapentin and CEAP} Complexes

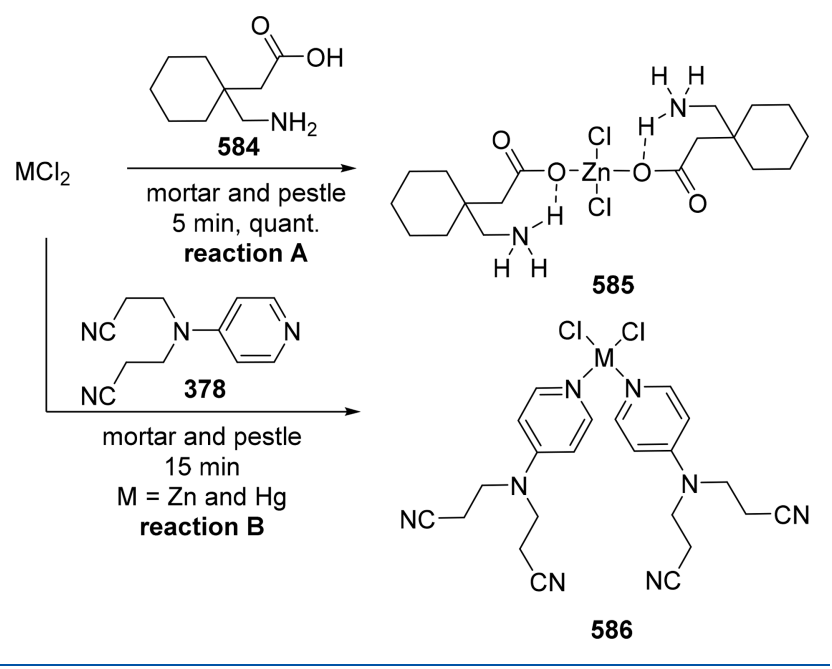

corresponding hydrogen-bonded salts $[\mathbf{C E A P H}]_{2}\left[\mathbf{M C l}_{4}\right](\mathrm{M}$ = zinc or mercury) were synthesized mechanochemically using CEAP 378 or CEAP. $\mathbf{H C l}$ as ligand source and $\mathrm{MCl}_{2}$ as metal source, respectively (Scheme 177 , reaction $\mathrm{B}){ }^{228}$ Interconversion of $\left[\mathbf{M C l}_{2}(\mathbf{C E A P})_{2}\right]$ and $[\mathrm{CEAPH}]_{2}\left[\mathbf{M C l}_{4}\right]$ was possible either through grinding in the presence of a base or through absorption of $\mathrm{HCl}$ vapors. In contrast to cobalt (Scheme 106) and zinc complexes, the mercury hydrogen-bonded salts could not be converted into the corresponding discrete $\left[\mathrm{HgCl}_{2}(\mathrm{CEAP})_{2}\right]$ complex by grinding with $\mathrm{KOH}$ in the solid state.
There is only one reported study on the synthesis of cadmium complexes using mechanochemistry. Grinding cadmium oxide with stoichiometric imidazole in an agate mortar provided the cadmium imidazolate complex in only 30 min. ${ }^{328}$ Similarly, only 20 min was necessary to prepare zinc or mercury imidazolates. Interestingly, zinc, cadmium, and mercury imidazolates prepared through grinding were found to have different polymorphic forms than the ones obtained by crystallization.

Crawford et al. recently used a batch mixer, previously used for the preparation of polymers, to optimize the reaction conditions for the synthesis of zinc complex 588 featuring 8hydroxyquinoline ligands (Scheme 178). ${ }^{329}$ Conditions

Scheme 178. Twin-Screw Extrusion of a Zinc Complex with OLED Applications

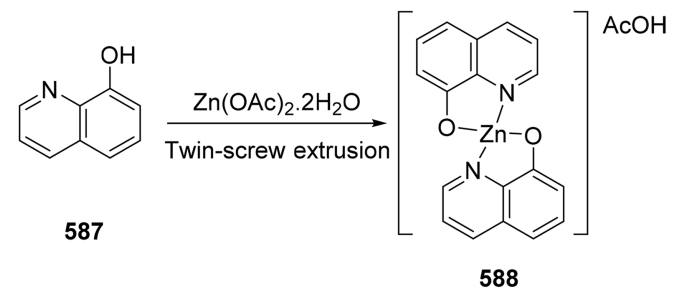

obtained were then directly transferred to twin-screw extrusion in order to scale-up the synthesis of the desired complex, which is of interest for OLED applications.

12.2. Microwave Irradiation for the Synthesis of Group 12 Metal Complexes

Microwave-assisted synthesis was used to form a series of cyanobipyridine-derived zinc(II) bis(thiolate) complexes 591. Two different procedures, with the addition of either an excess of bipyridine or a thioarene, were developed leading to different zinc complexes, 590 and 591, respectively (Scheme 179). ${ }^{330}$ Addition of a second bipyridyl unit provided the basic chromophoric framework $\mathbf{5 9 0}$ with the potential for modulating luminescent properties (Scheme 179, reaction 
Scheme 179. Synthesis of Zinc Complexes with Bipyridine Ligands under Microwave Irradiation

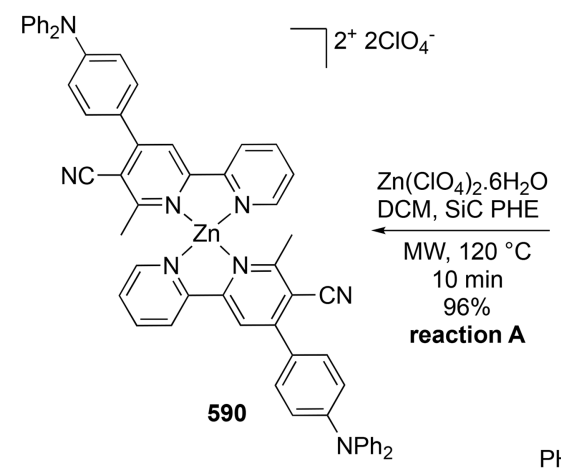

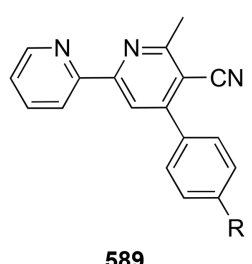

589

$\mathrm{PHE}=$ passive heating element
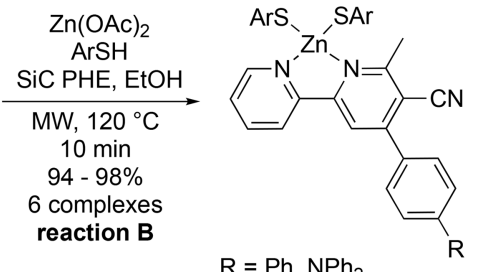

$\mathrm{R}=\mathrm{Ph}, \mathrm{NPh}_{2}$

$\mathrm{Ar}=$ napht, $\mathrm{Ph}, 4-\mathrm{MeOC}_{6} \mathrm{H}_{4}$

591

A). Under classical conditions, the desired complexes were obtained in a lower yield (44-56\%) and with higher time $(24$ h). Notably, the cyanobipyridine-derived zinc(II) bis(thiolate) complexes 591 displayed LLCT (ligand to ligand charge transfer) fluorescence in the solid state.

In order to optimize the synthesis of phthalocyanine-based zinc-containing liquid crystals, for which the main drawback is the rather long reaction time (from 22 to $88 \mathrm{~h}$ ), Akabane et al. have developed a microwave procedure, using ethylene glycol as the solvent, enabling the reaction time to be reduced to less than $1 \mathrm{~h}$ (Scheme 180). ${ }^{331}$ Five complexes $\mathbf{5 9 3}$ were synthesized in good yield (58-83\%) and characterized.

Scheme 180. Microwave Synthesis of Phthalocyanine Zinc Complexes
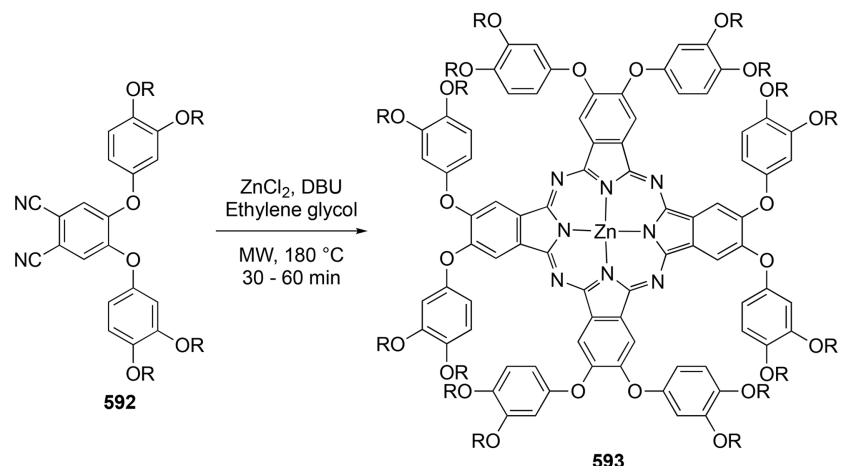

$\left[\mathrm{Zn}(79)_{2}\right]$ and $\left[\mathrm{Hg}(79)_{2}\right]$ complexes were formed by heating a solution of ligand 79 and $\mathrm{M}(\mathrm{OAc})_{2}(\mathrm{M}=\mathrm{Zn}$ and $\mathrm{Hg}$ ) in ethanol using microwave irradiation (Table 9, entries 1 and 2). ${ }^{133}$ Similarly, the synthesis of $[\mathbf{Z n}(87)]$ was described in 2009 using microwave irradiation starting from ligand 87 (Table 9, entry 3). ${ }^{135}$ Using $\mathrm{ZnCl}_{2}$ salt as the zinc source, $[\mathbf{Z n}(\mathbf{7 1})]^{134}$ and the macrocyclic $\left[\mathbf{Z n}(89)_{2}\right]^{236}$ were synthesized using microwave irradiation (Table 9, entries 4 and 5). The reaction mixture was first ground and then irradiated in a microwave oven in 3 or $4 \mathrm{~mL}$ of ethanol for a few minutes. All complexes were recovered after only a few seconds of heating and in higher yield than using conventional heating conditions.

A mixture of $\mathrm{Zn}\left(\mathrm{NO}_{3}\right)_{2} \cdot 4 \mathrm{H}_{2} \mathrm{O}$, 2-hydroxy-3-methoxybenzaldehyde, methylamine, and triethylamine in water was heated in a microwave oven (Table 9, entry 6) at $80{ }^{\circ} \mathrm{C}$ for $5 \mathrm{~min}$ (then rt for $50 \mathrm{~min})$ to produce $\left[\mathrm{Zn}_{7}(\mathrm{OH})_{6}(90)_{6}\right]\left(\mathrm{NO}_{3}\right)_{2}$ in $20 \%$ yield. ${ }^{136}$ Synthesis was also carried out in an autoclave, heating at $80{ }^{\circ} \mathrm{C}$ for $120 \mathrm{~h}$, and cooling to room temperature (period of $6 \mathrm{~h}$ ) to result in the desired complex in $15 \%$ yield. In contrast to $\left[\mathrm{Mn}_{7}(\mathrm{OH})_{6}(\mathbf{9 0})_{6}\right]\left[\mathrm{ClO}_{4}\right]_{2}$ (Table 5, entry 4), the zinc complex exhibited no fluorescent properties.

Four different Schiff base cadmium complexes were synthesized starting from a methanolic solution of chelates of carbazoles 93-96 derived from Schiff base ligands with $\mathrm{N}^{\wedge} \mathrm{X}$ $(\mathrm{X}=\mathrm{O}, \mathrm{S})$ link and $\mathrm{CdCl}_{2}$ (see Figure 1 for ligand structures). The reaction mixture was refluxed under microwave irradiation, and complexes were recovered in 5-6 min and in $89-92 \%$ yield (Table 9, entries $7-10) .{ }^{332}$ Interestingly, complexes were found more thermally stable than the corresponding ligands and inhibited the growth of microorganisms even at low concentrations.

Table 9. Schiff Base Used for the Synthesis of Zinc and Mercury Complexes ${ }^{a}$

\begin{tabular}{|c|c|c|c|c|c|c|}
\hline \multirow[b]{2}{*}{ entry } & \multirow[b]{2}{*}{ complex } & \multicolumn{2}{|c|}{ microwave synthesis } & \multicolumn{2}{|c|}{ conventional heating } & \multirow[b]{2}{*}{ ref } \\
\hline & & time $(\mathrm{min})$ & yield (\%) & time $(\mathrm{h})$ & yield (\%) & \\
\hline 1 & {$\left[\mathrm{Zn}(79)_{2}\right]$} & $15 \mathrm{~s}$ & 90 & 5 & 80 & 133 \\
\hline 2 & {$\left[\mathrm{Hg}(79)_{2}\right]$} & $25 \mathrm{~s}$ & 94 & 5 & 83 & 133 \\
\hline 3 & {$[\mathrm{Zn}(87)]$} & 1 & 93 & 5 & 84 & 135 \\
\hline 4 & {$[\mathrm{Zn}(71)] \cdot \mathrm{H}_{2} \mathrm{O}$} & 6 & 82 & 8 & 64 & 134 \\
\hline 5 & {$\left[\mathrm{Zn}(89)_{2}\right]$} & $8-10$ & $74-80$ & hours & 55 & 236 \\
\hline 6 & $\left.\left[\mathrm{Zn}_{7} \mathrm{OH}\right)_{6}(90)_{6}\right]\left(\mathrm{NO}_{3}\right)_{2}$ & 55 & 20 & 126 & 15 & 136 \\
\hline 7 & {$\left[\mathrm{Cd}(93)_{2} \mathrm{Cl}_{2}\right]$} & 6 & 92 & n.a. ${ }^{b}$ & n.a. ${ }^{b}$ & 332 \\
\hline 8 & {$\left[\mathrm{Cd}(94)_{2} \mathrm{Cl}_{2}\right]$} & 6 & 89 & n.a. ${ }^{b}$ & n.a. ${ }^{b}$ & 332 \\
\hline 9 & {$\left[\mathrm{Cd}(95)_{2} \mathrm{Cl}_{2}\right]$} & 5 & 90 & n.a. ${ }^{b}$ & n.a. ${ }^{b}$ & 332 \\
\hline 10 & {$\left[\mathrm{Cd}(96)_{2} \mathrm{Cl}_{2}\right]$} & 6 & 91 & n.a. ${ }^{b}$ & n.a. ${ }^{b}$ & 332 \\
\hline
\end{tabular}

${ }^{a}$ See Figure 1 for the structure of ligands. ${ }^{b}$ n.a.: not available. 
As for the synthesis of cobalt, nickel, and copper complexes featuring luminol derivatives as the ligand (Scheme 112), corresponding zinc(II) complex 594 was synthesized in less than $10 \mathrm{~min}$, in $81 \%$ yield starting from $\mathrm{ZnCl}_{2}$ (Scheme 181). The result in terms of fluorescence was found to be the same as with the other metals. The fluorescence lifetime was decreased compared to the free ligand. ${ }^{237}$

Scheme 181. Microwave-Assisted Synthesis of a ZincLuminol Derivative
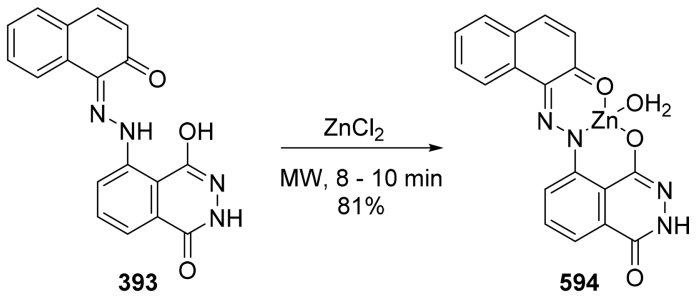

\subsection{Ultrasonic Activation and Mercury}

Two mercury(II) complexes 596 and 597 were synthesized from 4-mtrt ligand 595 (4-methyl-4H-1,2,4-triazole-3-thiol) and $\mathrm{HgX}_{2}(\mathrm{X}=\mathrm{Br}, \mathrm{Cl})$ under classical heating conditions or using an ultrasonic bath (Scheme 182). ${ }^{333^{\circ}}$ Sonochemical

\section{Scheme 182. Ultrasound Synthesis of Nanostructured} $\left[\mathrm{Hg}(4-\mathrm{mtrt}) \mathrm{X}_{2}\right]$ Complexes

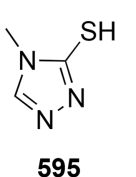

595

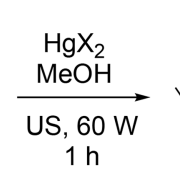

$\mathrm{h}$

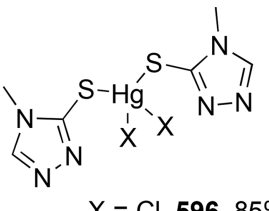

$\mathrm{X}=\mathrm{Cl}, \mathbf{5 9 6}, 85 \%$ $\mathrm{X}=\mathrm{Br}, 597,87 \%$

synthesis was carried out in order to obtain nanosized supramolecular materials, whereas synthesis in solution enabled crystals suitable for X-ray diffraction to be acquired. Comparison of PXRD patterns confirmed that both methods produced the same compounds; varying sizes of particles were, however, observed. SEM analysis showed that samples prepared by sonochemical methods were about 50-60 nm.

\section{MECHANOCHEMICAL ACCESS TO ALUMINUM AND INDIUM COMPLEXES}

Under solvent-free conditions, mechanochemical treatment of $\left[\mathrm{Al}(\mathrm{OAc})_{2}(\mathrm{OH})\right]$ and 8-hydroxyquinoline $\mathbf{5 8 7}$ led to the formation of $\mathrm{AlQ}_{3} \cdot \mathrm{AcOH} 598$ ( $\mathrm{Q}=8$-hydroxyquinolinate) within a few minutes (Scheme 183). ${ }^{334}$ Syntheses were developed in vibratory and planetary ball mills with scalability of up to $50 \mathrm{~g}$. Notably, $\mathbf{5 9 8}$ was easily transformed into $\mathrm{AlQ}_{3}$ by heating at $200{ }^{\circ} \mathrm{C}$ for $2 \mathrm{~h}$. Interestingly, another polymorph of 598 could be obtained via vapor diffusion of acetone into a solution of $\mathrm{AlQ}_{3}$ in acetic acid. The synthesis of $\mathbf{5 9 8}$ was recently described by using a batch mixer and a twin-screw extruder. ${ }^{329}$ The $\mathbf{A l Q}_{3}$ complex is well-known to exhibit photoluminescence and has important applications in OLED materials. Notably, samples obtained by solid-state synthesis exhibited identical photoluminescence as samples prepared by the conventional synthetic route.

Scheme 183. Ball-Mill Synthesis of $\mathrm{AlQ}_{3} \cdot \mathrm{AcOH}^{a}$

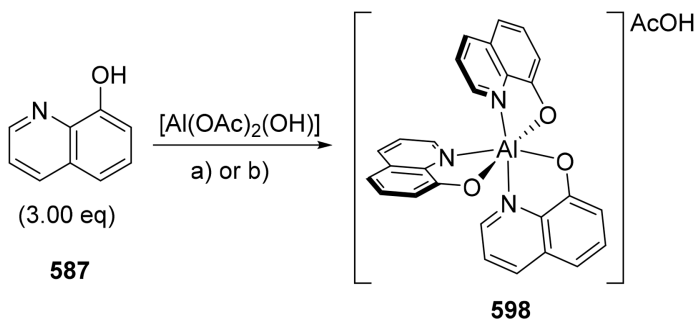

${ }^{a}$ Reaction conditions: (a) vbm, $25 \mathrm{~Hz}, 15 \mathrm{~min}, 94 \%$ (1.12 g), (b) pbm, $450 \mathrm{rpm}, 35 \mathrm{~min}, 78 \%$ (48.3 g).

Unsolvated tris(allyl)aluminum species had never been isolated under classical solution conditions, although adducts with $\mathrm{THF}, \mathrm{OPPh}_{3}$, and pyridine are known. In this context, Rightmire et al. described the mechanochemical synthesis of $\left[\mathrm{Al}\left(1,3-\left(\mathrm{SiMe}_{3}\right)_{2} \mathrm{C}_{3} \mathrm{H}_{3}\right)_{3}\right]$ starting from $\mathrm{AlX}_{3}(\mathrm{X}=\mathrm{Cl}, \mathrm{Br})$ and the allyl anion $\mathbf{5 9 9}$ (Scheme 184). ${ }^{80}$ Various milling processes were used; for example syntheses using a disperser mill led to the formation of around $150 \mathrm{mg}$ of $\left[\mathrm{Al}\left(1,3-\left(\mathrm{SiMe}_{3}\right)_{2} \mathrm{C}_{3} \mathrm{H}_{3}\right)_{3}\right]$ 600 in $15 \mathrm{~min}$ and in $80-85 \%$ yield. Using a planetary ball mill, $1.3 \mathrm{~g}$ of the complex was recovered in only $5 \mathrm{~min}$ with an excellent yield of $88 \%$. Interestingly, the tris(allyl)aluminum complex, featuring bulky ligands, did not react with sterically hindered triphenylmethanol but reacted instantaneously with benzophenone in solution to yield the tris(O-coordinated) aluminum species 601. Moreover, the nonsolvated aluminum species obtained through grinding was found to react faster than the THF-solvated one.

Indium complexes 604 and 605, featuring bisimine ligands 583, were prepared by mechanochemistry in 2016 by Wang et al. (Scheme 185). ${ }^{335}$ Alternatively, an efficient one-pot, twostep procedure starting from the corresponding diketone $\mathbf{6 0 2}$ and anilines 603 could be performed in a vibratory ball-mill MM400. The one-pot synthesis using 4-bromoaniline unfortunately led to the desired product in a mixture with other indium byproducts. Interestingly, the solid-state thermal reactions of neat bisimine with $\mathrm{InCl}_{3}$ at $180{ }^{\circ} \mathrm{C}$ for $2.5 \mathrm{~h}$ led to the decomposition of the starting materials without any reaction. With longer reaction times and lower yields, the same complexes could be obtained via classical solution conditions. The same protocol was later used for the synthesis of four more complexes featuring differently substituted bisimines. ${ }^{336}$

\section{GROUP 14}

\subsection{Mechanosynthesized Lead Complexes}

Due to their rapid progress in power conversion, perovskite photovoltaic devices have become alternative candidates for future power generation. In general, preparation methods of materials have an important influence on their properties. Numerous procedures have been developed including solvothermal syntheses and high-temperature solid-state reactions. Milling appropriate precursors using a mortar and pestle led to the formation of the perovskite with significant amounts of unreacted precursors. Using a vibratory ball mill for $30 \mathrm{~min}$, highly crystalline hydrid organic-inorganic perovskite $\mathrm{CH}_{3} \mathrm{NH}_{3} \mathrm{PbI}_{3}$ could be synthesized with full conversion starting from $\mathrm{CH}_{3} \mathrm{NH}_{3} \mathrm{I}$ and $\mathrm{PbI}_{2} \cdot{ }^{337}$ Particle analysis showed an exact stoichiometric composition and high thermal stability. In addition, the material exhibited superior performances 
Scheme 184. Mechanosynthesis of $\left[\mathrm{Al}\left(1,3-\left(\mathrm{SiMe}_{3}\right)_{2} \mathrm{C}_{3} \mathrm{H}_{3}\right)_{3}\right]$ Starting from Silylallyl anion ${ }^{a}$

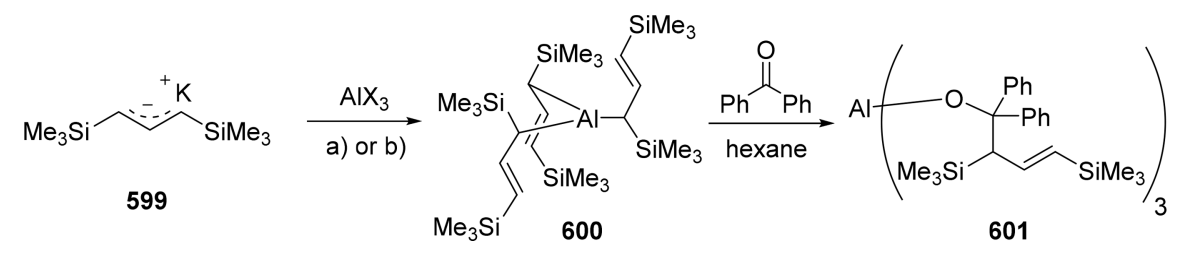

${ }^{a}$ Reaction conditions: (a) $\mathrm{X}=\mathrm{Cl}$ or $\mathrm{Br}$, disperser milling, $15 \mathrm{~min}, 80-85 \%$ (around $150 \mathrm{mg}$ scale), (b) $\mathrm{X}=\mathrm{Br}, \mathrm{pbm}, 600 \mathrm{rpm}, 5 \mathrm{~min}, 88 \%$ ( 1.3 gram scale).

Scheme 185. Mechanosynthesis of Indium Complexes

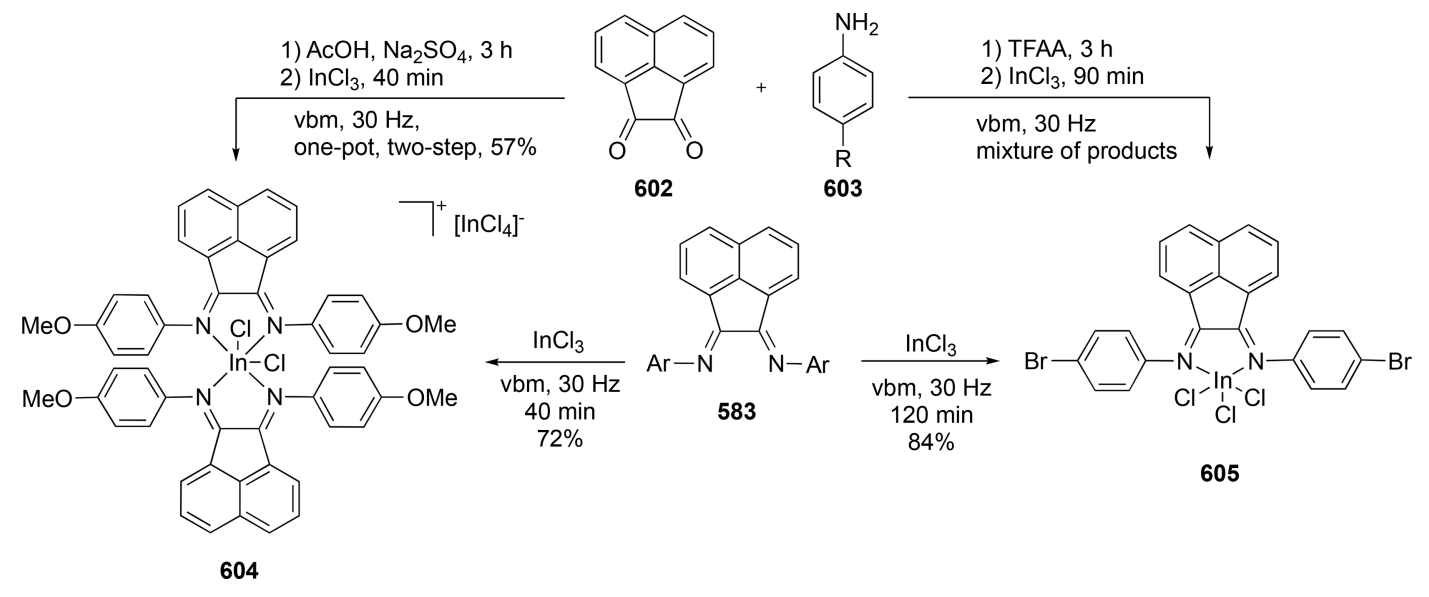

Scheme 186. Microwave-Assisted Synthesis of Tin Complexes of Hippuric Carboxylate

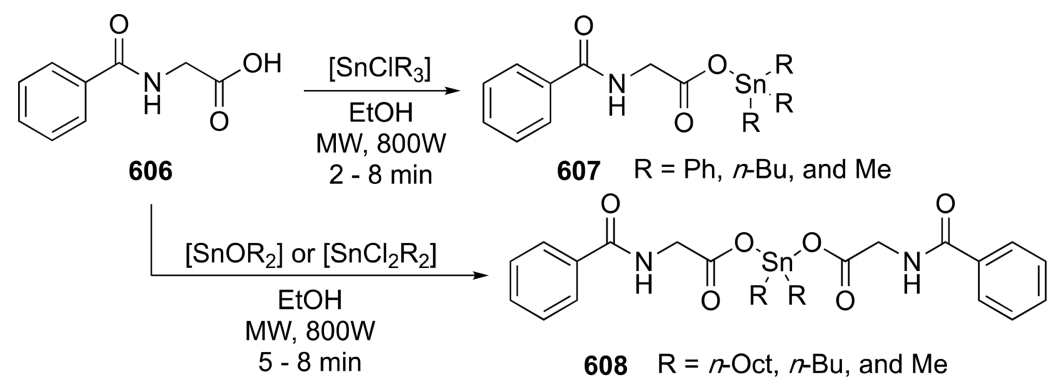

compared to perovskite prepared by a standard solution process.

\subsection{Microwave Syntheses of Tin and Germanium Compounds}

To evaluate their biological properties as anticancer and antiinflammatory agents, six different tin complexes featuring hippuric acid (HHA) 606 as the ligand were synthesized under microwave irradiation. ${ }^{338}$ Substitution of the chloride in various triorganotin(IV) chlorides $\mathrm{SnClR}_{3}$ resulted in the corresponding $\left[\mathbf{S n}(\mathbf{H A}) \mathbf{R}_{3}\right]$ complexes $607(\mathrm{R}=\mathrm{Ph}, n-\mathrm{Bu}$, and $\mathrm{Me})$. Alternatively, $\left[\mathrm{Sn}(\mathrm{HA})_{2} \mathbf{R}_{2}\right] 608(\mathrm{R}=n$-Oct, $n$-Bu, and $\mathrm{Me}$ ) could be obtained from dioorganotin chlorides or oxides (Scheme 186). As already described above for the synthesis of complexes featuring Schiff base ligands, the reaction mixture was ground with a mortar and pestle before irradiation. Comparison with the conventional thermal method demonstrated that the microwave irradiation method was more convenient because of a shorter reaction time (minutes vs 4 to $14 \mathrm{~h}$ under conventional conditions), and a lower quantity of solvent was used, although yields were comparable.
Starting from a solution of ligand 79 and $\mathrm{Sn}$ (II) acetate (at a stoichiometric ratio) in ethanol, $\left[\mathbf{S n}(79)_{2}\right]$ was synthesized via microwave irradiation in $35 \mathrm{~s}$ to obtain the product in $95 \%$ yield (Table 10 , entry 1 ). ${ }^{133}$ Similarly to cadmium complexes, four tin complexes were prepared from carbazole-derived Schiff base ligands (93-96) and $\mathrm{SnCl}_{2} \cdot 2 \mathrm{H}_{2} \mathrm{O}$. The corresponding complexes were recovered in only $5 \mathrm{~min}$ and in $89-96 \%$ yield (Table 10, entries 2-5). ${ }^{332}$

Microwave-assisted synthesis of eight organotin(IV) [Sn$\left.\left(\mathrm{CH}_{3}\right)_{2}(\mathrm{~L}) \mathrm{Cl}\right]$ and $\left[\mathrm{Sn}\left(\mathrm{CH}_{3}\right)_{2}(\mathrm{~L})_{2}\right]$ complexes was developed starting from the corresponding Schiff bases derived from dithiocarbazate (91 and 92), thiosemicarbazone (97 and 98), semicarbazone (99 and 100), or benzothiazoline (101 and 102) and $\mathrm{SnCl}_{2}\left(\mathrm{CH}_{3}\right)_{2}$ in solution in $\mathrm{MeOH} .{ }^{339,340}$ Equimolar and bimolar ratios of ligand/metal were used to obtain both families of complexes, $\left[\mathrm{Sn}\left(\mathrm{CH}_{3}\right)_{2}(\mathrm{~L}) \mathrm{Cl}\right]$ and $\left[\mathrm{Sn}\left(\mathrm{CH}_{3}\right)_{2}(\mathrm{~L})_{2}\right]$, respectively. In comparison with reflux, the use of microwave irradiation decreased reaction times from overnight to only a few minutes and increased the yield from $70-76 \%$ to $80-89 \%$ (Table 10, entries 6-21). All the tin complexes were tested and were found to be more active than their parent Schiff bases 
Table 10. Synthesis of Schiff-Base-Containing Tin Complexes $^{a}$

\begin{tabular}{|c|c|c|c|c|c|c|}
\hline \multirow[b]{2}{*}{ entry } & \multirow[b]{2}{*}{ tin complex } & \multicolumn{2}{|c|}{$\begin{array}{l}\text { microwave } \\
\text { procedure }\end{array}$} & \multicolumn{2}{|c|}{$\begin{array}{l}\text { classical } \\
\text { synthesis }\end{array}$} & \multirow[b]{2}{*}{ ref } \\
\hline & & $\begin{array}{l}\text { time } \\
(\mathrm{min})\end{array}$ & $\begin{array}{c}\text { yield } \\
(\%)\end{array}$ & $\begin{array}{l}\text { time } \\
(\mathrm{h})\end{array}$ & $\begin{array}{c}\text { yield } \\
(\%)\end{array}$ & \\
\hline 1 & {$\left[\operatorname{Sn}(79)_{2}\right]$} & 0.5 & 95 & 5 & & 133 \\
\hline 2 & {$\left[\mathrm{Sn}(93)_{2} \mathrm{Cl}_{2}\right]$} & 5 & 96 & n.d. ${ }^{a}$ & n.d. ${ }^{a}$ & 332 \\
\hline 3 & {$\left[\mathrm{Sn}(94)_{2} \mathrm{Cl}_{2}\right]$} & 5 & 96 & n.d. ${ }^{a}$ & n.d. ${ }^{a}$ & 332 \\
\hline 4 & {$\left[\mathrm{Sn}(95)_{2} \mathrm{Cl}_{2}\right]$} & 5 & 90 & n.d. ${ }^{a}$ & n.d. ${ }^{a}$ & 332 \\
\hline 5 & {$\left[\mathrm{Sn}(96)_{2} \mathrm{Cl}_{2}\right]$} & 5 & 89 & n.d. ${ }^{a}$ & n.d. ${ }^{a}$ & 332 \\
\hline 6 & {$\left[\mathrm{Sn}\left(\mathrm{CH}_{3}\right)_{2}(91) \mathrm{Cl}\right]$} & 8 & 84 & 15 & 76 & 339 \\
\hline 7 & {$\left[\mathrm{Sn}\left(\mathrm{CH}_{3}\right)_{2}(\mathbf{9 1})_{2}\right]$} & 6 & 82 & 16 & 73 & 339 \\
\hline 8 & {$\left[\mathrm{Sn}\left(\mathrm{CH}_{3}\right)_{2}(92) \mathrm{Cl}\right]$} & 5 & 87 & 14 & 71 & 339 \\
\hline 9 & {$\left[\mathrm{Sn}\left(\mathrm{CH}_{3}\right)_{2}(92)_{2}\right]$} & 5 & 86 & 13 & 73 & 339 \\
\hline 10 & {$\left[\mathrm{Sn}\left(\mathrm{CH}_{3}\right)_{2}(101) \mathrm{Cl}\right]$} & 4 & 85 & 16 & 72 & 339 \\
\hline 11 & {$\left[\mathrm{Sn}\left(\mathrm{CH}_{3}\right)_{2}(101)_{2}\right]$} & 6 & 87 & 13 & 73 & 339 \\
\hline 12 & {$\left[\mathrm{Sn}\left(\mathrm{CH}_{3}\right)_{2}(102) \mathrm{Cl}\right]$} & 6 & 85 & 14 & 69 & 340 \\
\hline 13 & {$\left[\mathrm{Sn}\left(\mathrm{CH}_{3}\right)_{2}(102)_{2}\right]$} & 4 & 84 & 17 & 70 & 340 \\
\hline 14 & {$\left[\mathrm{Sn}\left(\mathrm{CH}_{3}\right)_{2}(97) \mathrm{Cl}\right]$} & 3 & 82 & 15 & 75 & 340 \\
\hline 15 & {$\left[\mathrm{Sn}\left(\mathrm{CH}_{3}\right)_{2}(97)_{2}\right]$} & 6 & 86 & 15 & 72 & 340 \\
\hline 16 & {$\left[\mathrm{Sn}\left(\mathrm{CH}_{3}\right)_{2}(98) \mathrm{Cl}\right]$} & 5 & 89 & 14 & 70 & 340 \\
\hline 17 & {$\left[\mathrm{Sn}\left(\mathrm{CH}_{3}\right)_{2}(\mathbf{9 8})_{2}\right]$} & 5 & 87 & 13 & 73 & 340 \\
\hline 18 & {$\left[\mathrm{Sn}\left(\mathrm{CH}_{3}\right)_{2}(99) \mathrm{Cl}\right]$} & 4 & 85 & 16 & 71 & 340 \\
\hline 19 & {$\left[\mathrm{Sn}\left(\mathrm{CH}_{3}\right)_{2}(99)_{2}\right]$} & 6 & 88 & 13 & 69 & 340 \\
\hline 20 & {$\left[\mathrm{Sn}\left(\mathrm{CH}_{3}\right)_{2}(100) \mathrm{Cl}\right]$} & 6 & 80 & 14 & 74 & 340 \\
\hline 21 & {$\left[\mathrm{Sn}\left(\mathrm{CH}_{3}\right)_{2}(100)_{2}\right]$} & 4 & 84 & 15 & 70 & 340 \\
\hline
\end{tabular}

${ }^{a}$ See Figure 1 for the structure of ligands.

against several strains of bacteria (Bacillus subtilis(+), Staphylococcus aureus (+), Pseudomonas aeruginosa (-), and Salmoneall typhi $(-)$ ) and fungi (Penicillium crysogenes and Rhizoctonia phaseoli). Antifertility activity in male albino rats was also interesting, reducing the motility and the sperm count irreversibly.

Similarly, microwave irradiation was applied in the synthesis of germanium complexes $\mathbf{6 1 0}$ featuring ketimine ligands starting from a solution of $\left[\mathrm{GeClR}_{3}\right](\mathrm{R}=\mathrm{Me}$ and $\mathrm{Ph})$ and ketamine 609 in methanol (Scheme 187). ${ }^{341}$ Microwave

Scheme 187. Microwave-Assisted Synthesis of Schiff Base Germanium(IV) Complexes

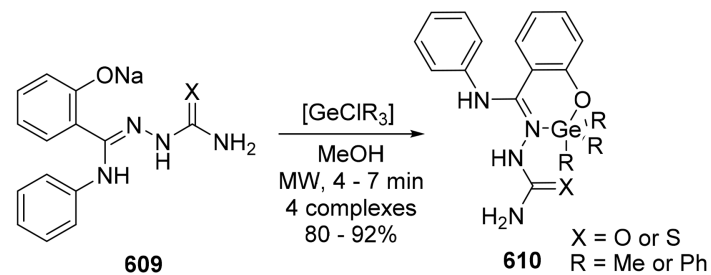

irradiation allowed the formation of the corresponding complexes in lower time and in higher yield (4-7 min, 80$92 \%)$ than under classical reflux conditions (12-15 h, 70$76 \%)$. These complexes showed moderate biological activity, however, higher than that displayed by the respective ligand, on selected fungi (Alternatria alternata, Aspergillus niger, Fusarium oxysporum, and Macrophomina phaseolina) and four bacteria (Staphylococcus aureus, Klebsiella aerogenes, Escherichia coli, and Pseudomonas cepacicola). Interestingly, the triphenylhydrazine carbothioamide germanium complex was highly effective as an insecticide with a $\mathrm{LC}_{50}$ (lethal concentration required to result in death of $50 \%$ of a test population) of 210 $\mathrm{mg} / \mathrm{L}$.

14.3. Ultrasonic Bath for the Synthesis of Lead Complexes

Two nanostructured lead(II) complexes of quinolone-2carboxylic acid $613^{342}$ and 2-methyl-8-hydroxyquinoline $611^{343}$ were prepared by the Davarani group using either electrochemistry or sonoelectrochemistry (Scheme 188). Nanosized samples were obtained thanks to a combination of electrochemistry and powerful ultrasonic radiation (20 $\mathrm{kHz}-10 \mathrm{MHz}$ ). At the cathode, the acidic proton in quinoline was reduced into $\mathrm{H}_{2}$, also yielding the anionic ligand, while oxidation of the $\mathrm{Pb}$ anode produced cationic $\mathrm{Pb}^{2+}$. It was proposed that the collapsing bubbles caused by ultrasound resulted in particles of high velocities that would push the nanoparticles to form nanospheres. Calcination of the resulting complex at $600{ }^{\circ} \mathrm{C}$ in air yielded $\mathrm{PbO}$ nanoparticles, which were found to be smaller than the $\mathrm{PbO}$ particles resulting from a calcination of the $\mathrm{Pb}$ (II) complex prepared through electrochemistry.

As already described above in the mechanochemical section, the $\mathrm{CH}_{3} \mathrm{NH}_{3} \mathrm{PbI}_{3}$ perovskite has become an alternative candidate for future power generation from sunlight. To synthesize this complex, two similar methods were developed at approximately the same time using ultrasound irradiation. In the first one, addition of a solution of $\mathrm{CH}_{3} \mathrm{NH}_{3} \mathrm{I}$ into a homogeneous dispersion of $\mathrm{PbI}_{2}$ in isopropanol (prepared using ultrasonic irradiation) resulted in the desired perovskite with a rod-like structure. ${ }^{344}$ In the second method, the addition of reagents was reversed, and the resulting reaction mixture was irradiated for $10-30 \mathrm{~min}$, yielding ultrafine polygonal nanoparticles $(10-40 \mathrm{~nm}) .^{345}$

\section{GROUP 15: SYNTHESES BY BALL MILLING}

Similarly to aluminum complexes (Scheme 184), grinding trihalides of $\mathrm{As}$ and $\mathrm{Sb}$ with allyl anion 599 led to the formation of tris(TMS-allyl)metal complexes $\mathbf{6 1 5}$ in only 5 $\min$ and in good yields (Scheme 189 , reaction A). ${ }^{346}$ Unfortunately, similar synthesis using bismuth(III) halide salt led to a dark gray powder, which consisted of bismuth metal and hexadiene as the only identifiable products. An alternative strategy was to mill tris(allyl)aluminum complex 600 with

\section{Scheme 188. Sonochemical Synthesis of Lead(II) Complexes of O-,N-Coordinating Ligand}

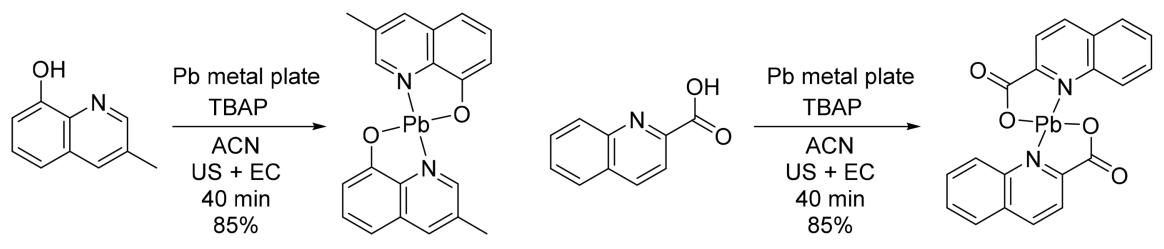


Scheme 189. Mechanochemical Synthesis of Tris(TMS-allyl)metal Complexes

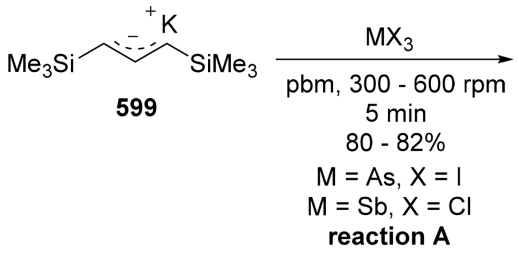

reaction $A$

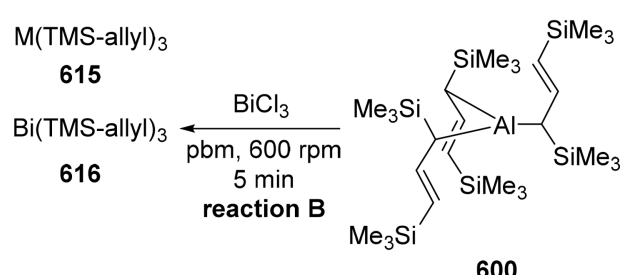

600
$\mathrm{BiCl}_{3}$ in a planetary ball mill for $5 \mathrm{~min}$. The desired tris(TMSallyl)Bi complex 616 was formed albeit as a mixture with unidentified byproducts (Scheme 189, reaction B). In addition, this bismuth complex was found to be light-sensitive and thermally unstable and had completely decomposed within 3 days at room temperature. Complexes were isolated as a mixture of diastereoisomers of $C_{1}$ and $C_{3}$ symmetry, with the ratio of $C_{1} / C_{3}$ dependent on the metal. Notably, using appropriate conditions (solvent and reagents), all complexes formed with longer reaction times $(2-72 \mathrm{~h}$ ) and in lower yields $(64-80 \%)$ in solution. The bismuth complex described above was not the first bismuth complex to be synthesized by ball milling. Indeed, the synthesis of various ortho-functionalized triarylbismuthanes by ball milling was reported in 2003 by the Suzuki group. ${ }^{347}$ In contrast to the conventional solution method, this ball-milling approach was simple and efficient and produced the complex in satisfactory yields.

\section{LANTHANIDE}

\subsection{Mechanochemical Approach}

Inspired by the original synthesis of $\left[\mathrm{Sm}\left(\mathrm{Cp}^{*}\right)_{3}\right]$, analogous lanthanide complexes were prepared from $\mathrm{Ln}^{3+}$ precursors (La, $\mathrm{Ce}, \mathrm{Pr}, \mathrm{Nd}$, and $\mathrm{Gd}$ ) in benzene or in toluene. Starting from heavy lanthanides, Woen et al. described the synthesis of the unknown $\left[\mathbf{L n}\left(\mathbf{C p}^{*}\right)_{3}\right](\operatorname{Ln}=\mathrm{Tb}, \mathrm{Dy}, \mathrm{Ho}$, and $\mathrm{Er})$ complexes 618. $^{348}$ Since steric bulk in $\left[\operatorname{Ln}\left(\mathbf{C p}^{*}\right)_{3}\right]$ with small metallic centers may result in arene (toluene, benzene) metalation, a solvent-free method was envisioned. Syntheses were performed in a disperser mill containing 40 stainless steel balls by reacting $\mathrm{KC}_{5} \mathrm{Me}_{5}$ with lanthanide $\left[\mathbf{L n}\left(\mathbf{C} \mathbf{p}^{*}\right)_{2}(\boldsymbol{\mu}-\mathbf{P h})_{2} \mathbf{B P h}_{2}\right]$ precursors 617, which contained a labile tetraphenylborate counteranion (Scheme 190). XRD analysis of the resulting complexes

Scheme 190. Syntheses of Lanthanide Complexes Using a Disperser Mill
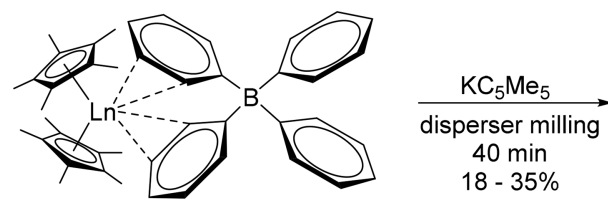

617

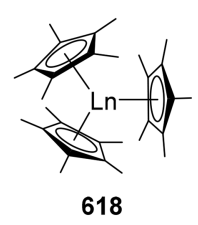

showed a tilt of a $\mathrm{Cp}^{*}$ ring away from the metal center. This tilt increases as the ionic radius of the metal decreases and was proposed as a metric to evaluate the ability of a $\left[\mathbf{L n}\left(\mathbf{C p}^{*}\right)_{3}\right]$ complex to achieve $\mathrm{C}-\mathrm{H}$ activation of arenes.

16.2. Microwave Syntheses

Kumari et al. described the solvent-free synthesis of different lanthanide complexes ( $\mathrm{Ln}=\mathrm{La}, \mathrm{Ce}, \mathrm{Pr}, \mathrm{Nd}, \mathrm{S}, \mathrm{Eu}$, and $\mathrm{Gd})$ featuring (2-( $N$-salicylideneamino)-3-carboxyethyl-4,5-dime-

thylthiophene $) \quad 619^{349}$ and ([(2-hydroxybenzaldehyde)-3isatin] bishydrazone) $\mathbf{6 2 0}^{350}$ Schiff base ligands under microwave irradiation. The ligand and metal were first ground with a mortar and pestle with ethanol, and the $\mathrm{pH}$ was adjusted to be between 6.0 and 6.5. The solvent was then evaporated, and the resulting reaction mixture was irradiated for $10-15 \mathrm{~min}$ to produce complexes $\mathbf{6 2 1}$ and $\mathbf{6 2 2}$ in $60-70 \%$ yield (Scheme 191). Lanthanide complexes exhibited greater in vitro antibacterial activity than their respective ligands against Escherichia coli, Staphylococcus aureus, Bacillus megaterium, and Vibrio cholera. Interestingly, the $\left[\mathrm{La}(619)_{2} \mathrm{Cl}_{3}\right]$ complex was subjected to transesterification in alcohol (methanol and $n$-propanol), although not through microwave activation, giving rise to two new complexes. Notably, antifungal activity was demonstrated to be dependent on the nature of the metal ion, the nature of the ligand, the coordinating sites, the geometry of the complex, the hydrophilicity, the lipophilicity, the presence of coligands, and also steric and pharmacokinetic factors.

$\left[\mathrm{Tb}(\text { acac })_{3}\right.$ (dmdpphen) 624 (dmdpphen $623=2,9$ dimethyl-4,7-diphenyl-1,10-phenanthrolinate), a complex exhibiting intense green luminescence, was synthesized under microwave irradiation. A solution of both ligands and terbium(III) nitrate in ethanol was heated under microwave irradiation for $12 \mathrm{~min}$ (Scheme 192). ${ }^{351}$ The resulting terbium complex 624, which demonstrated good thermal stability and high green luminescence, could be used in functional materials such as OLEDs.

In 2013, the Zhou group described the synthesis of two europium complexes featuring cinnamate ligands, $[\mathrm{Eu}(\mathrm{phen})$ $\left.\left(\mathrm{C}_{6} \mathrm{H}_{5} \mathrm{CH}=\mathrm{CHCOO}\right)_{3}\right] 625^{352}$ and $\left[\mathrm{Eu}(\right.$ bipy $)\left(\mathrm{C}_{6} \mathrm{H}_{5} \mathrm{CH}=\right.$ CHCOO $\left.)_{3}\right] 626$ (Scheme 193). ${ }^{353}$ Both ligands, bipyridine and cinnamic acid, were dissolved in EtOH and added to a solution of europium nitrate, and the $\mathrm{pH}$ of the resulting solution was adjusted to $6-7$ using a $6 \mathrm{M} \mathrm{NH}_{3}$ aqueous solution. Subsequently, the reaction mixture was irradiated for 20-24 $\mathrm{min}$ in a microwave oven. Both complexes exhibited high luminescence at $614 \mathrm{~nm}$ for $\mathbf{6 2 5}$ and at 592, 615, and 697 nm for 626.

\section{CONCLUSION}

The synthesis of organometallic complexes using enabling technologies has already attracted a lot of attention, and this field still has a bright future. Until recently, a special focus was placed on the synthesis of discrete metal complexes featuring palladium, copper, or iron as the metal center, mostly because of their catalytic properties. Few metals have been investigated until now, for example, titanium, osmium, or iridium. The aim of this review was to provide the chemistry community with an overview of the possibilities, opportunities, and benefits of using enabling technologies. Most importantly, by underlying understudied areas, it opens perspectives for the future of 
Scheme 191. Microwave-Assisted Synthesis of Lanthanide Complexes
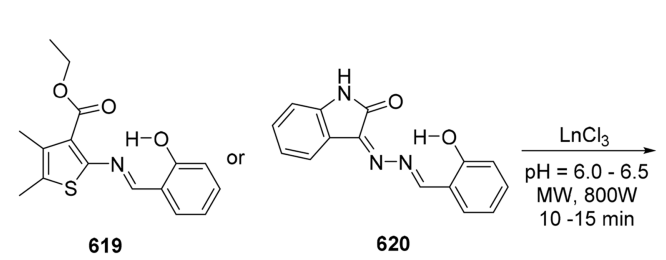

620
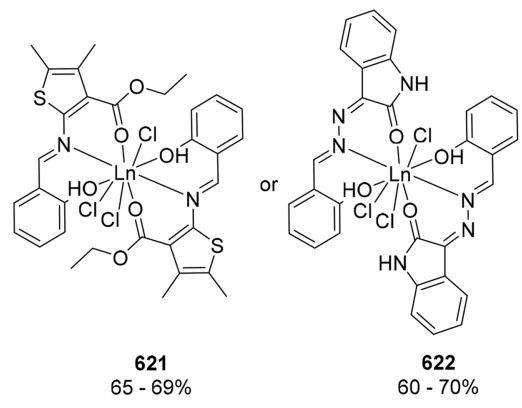

Scheme 192. Microwave-Assisted Synthesis of a Highly Luminescente Terbium(III) Complex

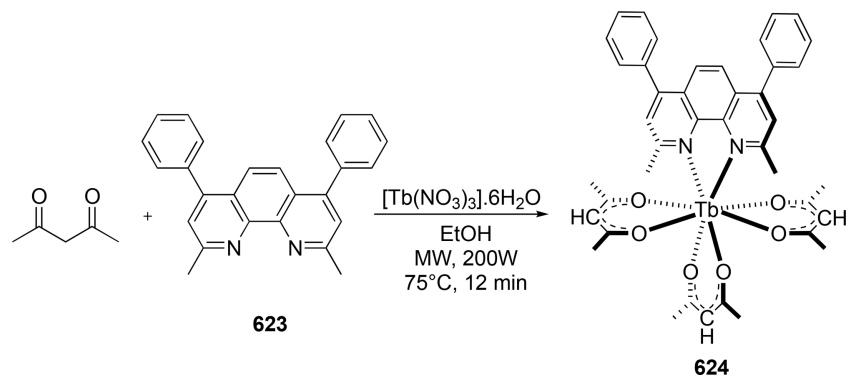

Scheme 193. Synthesis of Europium Complexes with Cinnamic Acid

$$
\begin{array}{cc}
\mathrm{Eu}\left(\mathrm{NO}_{3}\right)_{3} \cdot \mathrm{nH}_{2} \mathrm{O} \quad \begin{array}{l}
\text { acid cinamic }(3 \mathrm{eq}) \\
\text { bipy or phen }(1 \mathrm{eq})
\end{array} \\
\begin{array}{c}
\text { EtOH } \\
\mathrm{MW}, 600 \mathrm{~W} \\
20-24 \mathrm{~min}
\end{array}
\end{array}
$$

$\left[\mathrm{Eu}(\right.$ phen $\left.)(\mathrm{PhCHCHCOO})_{3}\right] 625$

[Eu(bipy) $\left.(\mathrm{PhCH} \text { CHCOO})_{3}\right] 626$

organometallic synthesis. It is clear that the habits of chemists, persistent for more than one century, in using a classical flask with a stirring plate need to be modified. It is now time to evolve to perform chemistry faster and more efficiently.

Microwave activation, mechanochemistry, ultrasonic activation, photochemistry, and continuous flow chemistry clearly bring new ways of thinking about chemistry and provide new opportunities to access compounds, which would not be reachable in any other way. Microwave equipment, mostly used to reduce reaction times while increasing pressure and temperature, is widely available in laboratories and could be used more systematically.

Mechanochemistry, mostly through ball milling, is becoming more and more prevalent. In this review, most of the examples were performed using a mortar and pestle, the simplest equipment available. This technology still has a bright future since only specialized research groups are currently equipped, and among them few are taking organometallic synthesis into account.

Ultrasound is less commonly used in organometallic synthesis. This is probably because this activation method is not widespread. Nevertheless, this approach is highly interesting in terms of understanding how cavitation can promote reactions.

Photochemistry has been used mostly for ligand exchange or modification, with a wide range of metals. This activation is becoming more and more established in organic chemistry, notably with photoredox catalysis, but its use in organometallic synthesis or modification is still underused. There is no doubt that, with the development of photochemistry in general, the applications in organometallic synthesis will increase.

Finally, continuous flow chemistry is highly interesting in terms of safety, scalability, and reactivity. However, it is still in its infancy for organometallic synthesis, with very rare examples of discrete metal complexes synthesized in a continuous flow process. Flow chemistry was initially expensive, which caused reluctance in researchers to use this technique; the equipment has, however, recently become much more affordable and modular. The benefits in performing flow chemistry should motivate organometallic chemists to turn their attention to this novel technology.

It is also important to add that the technologies presented herein mostly contribute to one of the most important topics of the 21st century, namely, respect for the environment. Reduction of reaction times, of the quantity of solvent used, and of the formation of side products and safer procedures are all qualities that fit the 12 principles of Green Chemistry developed in 1992 by Anastas and Warner. There is thus no doubt that enabling technologies will be part of the future of organometallic synthesis.

\section{AUTHOR INFORMATION}

\section{Corresponding Authors}

*E-mail: xavier.bantreil@umontpellier.fr.

*E-mail: thomas-xavier.metro@umontpellier.fr.

*E-mail: frederic.lamaty@umontpellier.fr.

ORCID $\odot$

Xavier Bantreil: 0000-0002-2676-6851

Thomas-Xavier Métro: 0000-0003-2280-3595

Jean Martinez: 0000-0002-4551-4254

Frédéric Lamaty: 0000-0003-2213-9276

Notes

The authors declare no competing financial interest.

\section{Biographies}

Audrey Beillard graduated with a degree in fine and industrial organic chemistry from the Ecole Nationale Supérieure d'Ingénieur de Caen (ENSICAEN) in 2014. The same year, she also obtained a Master's degree in organic synthesis at the University of Caen. She received her Ph.D. in biomolecular engineering from the University of Montpellier (Institute of Biomolecules Max Mousseron) in 2017, under the direction of Dr. Frédéric Lamaty, on the mechanochemical synthesis of NHC-metal complexes. In 2018, she joined the Commissariat à l'Energie Atomique et aux Energies Alternatives (CEA) of Marcoule in France as a postdoctoral researcher, where she worked on the synthesis of new extractants for liquid-liquid extraction. 
Xavier Bantreil graduated as a chemical engineer in 2004 from Ecole Nationale Supérieure de Chimie de Paris (ENSCP-ParisTech, France). He then received a Master's degree and later his Ph.D. in 2008 from Université Pierre et Marie Curie (UPMC - Paris VI) under the supervision of Prof. Giovanni Poli, working on palladiumcatalyzed reactions. During his doctorate, he spent two months in the group of Prof. Paul S. Pregosin at ETH Zürich working on the isolation of $\pi$-allyl palladium complexes. He then took up a postdoctoral position in the group of Profs. Steven P. Nolan and Catherine S. J. Cazin and spent three months at the ICIQ in Tarragona (Spain) and then 19 months at the University of St Andrews (UK), working on the synthesis of new catalysts for olefin metathesis. He then returned to France for a one-year postdoctoral position in Dr. Philippe Belmont's research group at Institut Curie (Paris, France) working on the silver-catalyzed synthesis of quinoline derivatives. In September 2011, he joined Dr. Frédéric Lamaty's group in Montpellier at the Institut des Biomolécules Max Mousseron (IBMM) as assistant professor. In 2017, he obtained his habilitation (HDR). His research topics are focused on catalysis, organometallic synthesis, and organic and green chemistry, with a special emphasis on heterocycles and organometallics and the use of alternative solvents and enabling technologies (microwaves, ball milling, continuous flow).

After having spent one year at Atofina Chemicals, USA, working on organic peroxide safety process research, Thomas-Xavier Métro graduated with both a Master's degree in general chemistry from the Ecole Supérieure de Chimie Organique et Minérale and a Master's degree in medicinal chemistry from the Université de Pharmacie de Montpellier in 2004. He completed his Ph.D. in organic chemistry in 2008 at the Universite Pierre et Marie Curie (Paris) under the supervision of Dr. Domingo Gomez Pardo and Prof. Janine Cossy, working on the rearrangement of amino alcohols and its application to the synthesis of $(S, S)$-reboxetine and SSR241586. After a first postdoc in process R\&D at Sanofi Chimie in Sisteron and a second postdoc in medicinal chemistry at Sanofi-Aventis R\&D in Montpellier, he joined the CNRS in 2011 as a Chargé de Recherche in the Green Chemistry and Enabling Technologies Team at the Max Mousseron Institute for Biomolecules (IBMM; Montpellier). Since then, his research activity has mainly been based on the development of solvent-free/solventless methodologies, aiming to provide the organic chemist with a set of solutions to reduce the environmental impact of chemical enterprise.

Jean Martinez received his Ph.D. degree in organic chemistry from the University of Montpellier at the Ecole Nationale Supérieure de Chimie de Montpellier under the direction of Prof. F. Winternitz. He completed his chemical education as a postdoctoral fellow with Dr. E. Bricas, Orsay, University of Paris Sud, and then with Prof. M. Bodanszky, Case Western Reserve University, Cleveland, OH. Back in France, he was recruited as a CNRS Research Associate and then as a Research Director in Montpellier. Pursuing investigations at the interface of chemistry and biology, he joined the University of Montpellier and was appointed Full Professor in both organic chemistry and medicinal chemistry. He became the head of a succession of research laboratories in Montpellier including the Laboratory of Chemistry and Pharmacology of Biologically Interesting Molecules, the Laboratory of Amino Acids, Peptides and Proteins. He founded the Institute of Biomolecules Max Mousseron in 2007 and acted as the director until December 2014. In 2015, he remained in the same institute, taking up the position of head of the Department of Amino Acids, Peptides and Proteins. His current research interests focus on peptide chemistry, stereoselective syntheses of biomolecules, design and production of original biomaterials, green chemistry, and the biology and pharmacology of neuropeptides of the gastrointestinal tract.

Frédéric Lamaty graduated as a chemical engineer in 1988 from the Ecole Supérieure de Chimie Industrielle de Lyon (ESCIL now ESCPE, France). In 1992, he received his Ph.D. degree from Purdue University (West Lafayette, IN, USA) under the supervision of Professor Ei-ichi Negishi in the field of Pd-catalyzed cyclizations. He then joined the group of Professor Marc Julia at the Ecole Normale Supérieure in Paris as a Rhône-Poulenc postdoctoral fellow to work on the synthesis of Vitamin A. In 1994, he obtained a permanent position at the Centre National de la Recherche Scientifique (CNRS) in Montpellier and is currently working as Directeur de Recherche at the Max Mousseron Institute for Biomolecules (IBMM). Since 2011, he has headed the Green Chemistry and Enabling Technologies team (www.greenchem.um2.fr) at IBMM. His research topics in the area of catalysis, organic, and green chemistry are devoted to the synthesis of amino acids, peptides, heterocycles, and organometallics, the use of alternative solvents (PEG, PEG-IL, glycerol, water), and enabling technologies (microwaves, ultrasound, ball milling, continuous flow) with an emphasis on mechanochemistry. In 2011 he was awarded the Prix des Techniques Innovantes pour l'Environnement ADEMEPollutec for his activity in peptide mechanosynthesis under solventfree conditions.

\section{ACKNOWLEDGMENTS}

The Université de Montpellier, Centre Nationale de la Recherche Scientifique (CNRS), French Ministry of Higher Education and Research (grant to A. B.), and Agence Nationale de la Recherche (grant no. ANR-16-CE07-0009-01 for X. B.) are acknowledged for funding.

\section{LIST OF ABBREVIATIONS}

$\begin{array}{ll}\text { \% de } & \text { percentage diastereomeric excess } \\ \text { 9-atc } & \text { 9-anthracenecarboxylic acid } \\ \text { acac } & \text { acetylacetonate } \\ \text { ACIM } & \text { 4-imino-2-pentanonate } \\ \text { ACN } & \text { acetonitrile } \\ \text { BIAN } & \text { bis(arylimino)acenaphthene } \\ \text { Bipy } & \text { bipyridine } \\ \text { Bppz } & \text { 2,3-bis(2-pyridyl)pyrazine } \\ \text { bqn } & 2,2^{\prime} \text {-biquinoline } \\ \text { CEAP } & 4 \text {-[N,N-bis(2-cyanoethyl)amino]pyridine } \\ \text { CF } & \text { continuous flow } \\ \text { COD } & \text { cycloocta-1,5-diene } \\ \text { Cp } & \text { cyclopentadienyl } \\ \text { Cp' } & \text { methylcyclopentadienyl } \\ \text { Cp* } & \text { pentamethylcyclopentadienyl } \\ \text { Cp^ } & \text { "Cp roof ligand” } 2,4 \text {-di-tert-butyl-3-(2,2-dimethyl- } \\ & \text { propyl)-1-methoxycyclopentadienyl } \\ \text { DBU } & 1,8 \text {-diazabicyclo[5.4.0]undec-7-ene } \\ \text { dcbpy } & 4,4^{\prime} \text {-dicarboxy-2,2'-bipyridine } \\ \text { DCM } & \text { dichloromethane } \\ \text { dcmb } & 4,4^{\prime} \text {-dimethoxycarbonyl-2,2'-bipyridine } \\ \text { debpy } & 4,4^{\prime} \text {-diethoxycarbonyl-2,2'-bipyridine } \\ \text { deeb } & 4,4^{\prime} \text {-diethylester-2,2'-bipyridine } \\ \text { detu } & \text { N,N'-diethylthiourea } \\ \text { diag- } & \text { diagonal } \\ \text { DIOP } & 2,3 \text {-O-isopropylidene-2,3-dihydroxy-1,4-bis- } \\ & \text { (diphenylphosphino)butane } \\ \text { dmbpy } & 4,4^{\prime} \text {-dimethyl-2,2'-bipyridine } \\ \text { dmdpphen } & 2,9 \text {-dimethyl-4,7-diphenyl-1,10-phenanthrolinate } \\ \text { DME } & 1,2 \text {-dimethoxyethane } \\ & \end{array}$




\begin{tabular}{|c|c|c|c|}
\hline DMF & $N, N$-dimethylformamide & phbn & 3-phenylbenzo $[\mathrm{b}][1,6]$ naphthyridine \\
\hline DMSO & dimethyl sulfoxide & PHE & passive heating element \\
\hline DNA & desoxyribonucleic acid & Phen & 1,10-phenanthroline \\
\hline dpbpy & $4,4^{\prime}$-diphenyl-2,2'-bipyridine & phphen & 5-phenyl-1,10-phenanthroline \\
\hline dpephos & bis [(2-diphenylphosphino)phenyl] ether & phq & 2-phenyl-1-quinoline \\
\hline DPM & 2,2,6,6-tetramethyl-3,5-heptadionato & pip & piperidine \\
\hline dppb & 1,4-bis(diphenylphosphino)butane & PMMA & poly(methyl methacrylate) \\
\hline dppe & 1,2-bis(diphenylphosphino)ethane & PMP & para-methoxyphenyl \\
\hline dppf & $1,1^{\prime}$-bis(diphenylphosphino)ferrocene & $\operatorname{ppfp}_{3}$ & tris(pentafluorophenyl)phosphine \\
\hline dpphen & 4,7-diphenyl-1,10-phenanthroline & PS & polystyrene \\
\hline dppm & bis(diphenylphosphino)methane & PTA & 1,3,5-triaza-7-phosphaadamantane \\
\hline dppp & 1,3-bis(diphenylphosphino)propane & PTFE & polytetrafluoroethylene \\
\hline dpypp & $2,2^{\prime}$-(phenylphosphinediyl)dipyridine & PXRD & powder X-ray diffraction \\
\hline dqp & 2,6-di(quinolin-8-yl)pyridine & Py & pyridine \\
\hline dqp- $\mathrm{NH}_{2}$ & 2,6-di(quinolin-8-yl)-4-aminopyridine & $\mathrm{pz}$ & pyrazole \\
\hline $\mathrm{dqpCO}_{2} \mathrm{Et}$ & 4-ethyl-carboxy-2,6-di(quinolin-8-yl)pyridine & pzpy & ethyl[3-(2-pyridyl)-1-pyrazolyl]acetate \\
\hline E. coli & Escherichia coli & Q & 8-hydroxyquinolinate \\
\hline en & 1,2-ethylenediamine & $\mathrm{saoH}_{2}$ & salicylaldoxime \\
\hline ER & estrogen receptor & SEM & scanning electron microscopy \\
\hline $\begin{array}{l}\text { ettu } \\
\text { etu }\end{array}$ & $\begin{array}{l}N \text {-ethylthiourea } \\
\text { ethylenethiourea }\end{array}$ & SIMes $\cdot \mathrm{HCl}$ & $\begin{array}{l}\text { 1,3-bis }(2,4,6 \text {-trimethylphenyl })-1 H \text {-imidazolinium } \\
\text { chloride }\end{array}$ \\
\hline $\begin{array}{l}\text { fac- } \\
\text { Fc }\end{array}$ & $\begin{array}{l}\text { facial } \\
\text { ferrocenyl }\end{array}$ & $\mathrm{SIPr} \cdot \mathrm{HCl}$ & $\begin{array}{l}\text { 1,3-bis }(2,6 \text {-diisopropylphenyl)- } 1 H \text {-imidazolinium } \\
\text { chloride }\end{array}$ \\
\hline $\mathrm{H}_{2}$ cit & dihydrogencitrate & SOD & superoxide dismutase \\
\hline $\mathrm{H}_{2} \mathrm{MSAP}$ & $\begin{array}{l}N \text {-3-methoxysalicylidene-2-amino-3-hydroxypyri- } \\
\text { dine }\end{array}$ & $\begin{array}{l}\text { SQ } \\
\text { tbbpy }\end{array}$ & $\begin{array}{l}\text { semiquinonate } \\
4,4^{\prime} \text {-di-tert-butyl-2,2'-bipyridine }\end{array}$ \\
\hline HHA & hippuric acid & $t$ BuXPhos & di-tert-butyl $\left(2^{\prime}, 4^{\prime}, 6^{\prime}\right.$-triisopropylbiphenyl-2-yl)- \\
\hline Him & imidazole & & phosphine \\
\hline $\mathrm{Hpz}$ & pyrazole & TCB & 1,2,4-trichlorobenzene \\
\hline $\mathrm{IC}_{50}$ & half maximal inhibitory concentration & tctpy & tricarboxylterpyridine \\
\hline $\mathrm{IEt}_{2} \mathrm{Me}_{2}$ & 1,3-diethyl-4,5-dimethylimidazol-2-ylidene & TEAB & tetraethylammonium bromide \\
\hline IMes $\cdot \mathrm{HCl}$ & $\begin{array}{l}\text { 1,3-bis }(2,4,6 \text {-trimethylphenyl }) \text { - } 1 H \text {-imidazolium } \\
\text { chloride }\end{array}$ & $\begin{array}{l}\text { terpy } \\
\text { THF }\end{array}$ & $\begin{array}{l}2,2^{\prime}, 2^{\prime \prime} \text {-terpyridine } \\
\text { tetrahydrofuran }\end{array}$ \\
\hline Ind & indene & TIPSA & triisopropylsilylacetylene \\
\hline $\mathrm{IPr}$ & 1,2-bis(2,6-diisopropylphenyl)imidazol-2-ylidene & tipsepd & ((triisopropylsilyl)ethynyl)-2,4-pentanedionate \\
\hline $\mathrm{IPr} \cdot \mathrm{HCl}$ & 1,3-bis(2,6-diisopropylphenyl)- $1 H$-imidazolium & & ion \\
\hline & chloride & TMEDA & $N, N, N^{\prime}, N^{\prime}$-tetramethylethylenediamine \\
\hline IR & infrared & TMP & trimethoprim (5-(3,4,5-trimethoxybenzyl)- \\
\hline JohnPhos & di (tert-butyl $)\left(1,1^{\prime}\right.$-biphenyl-2-yl)-phosphine & & pyrimidine-2,4-diamine) \\
\hline KbpzB & potassium bispyrazolyl borate & TMS & trimethylsilyl \\
\hline LAG & liquid-assisted grinding & tosyl & 4-methylbenzene-1-sulfonyl \\
\hline lat- & lateral & $\mathrm{Tp}$ & hydrido-tris(pyrazolyl)borate \\
\hline LED & light-emitting diode & TPBP & 1,3,5-tris(2-(pyridin-4-yl)vinyl)benzene \\
\hline LLCT & ligand to ligand charge transfer & tterpy & $4^{\prime}$-(4-tolyl)-2,2':6',2”-terpyridine \\
\hline MAS & magic-angle spinning & tu & thiourea \\
\hline mer- & meridional & US & ultrasound \\
\hline MLCT & metal to ligand charge transfer & UV & ultraviolet \\
\hline morph & morpholine & vbm & vibratory ball mill \\
\hline MTBE & methyl tert-butyl ether & W & Watt \\
\hline MW & microwave & & \\
\hline NBS & $N$-bromosuccinimide & \multicolumn{2}{|c|}{ REFERENCES } \\
\hline
\end{tabular}

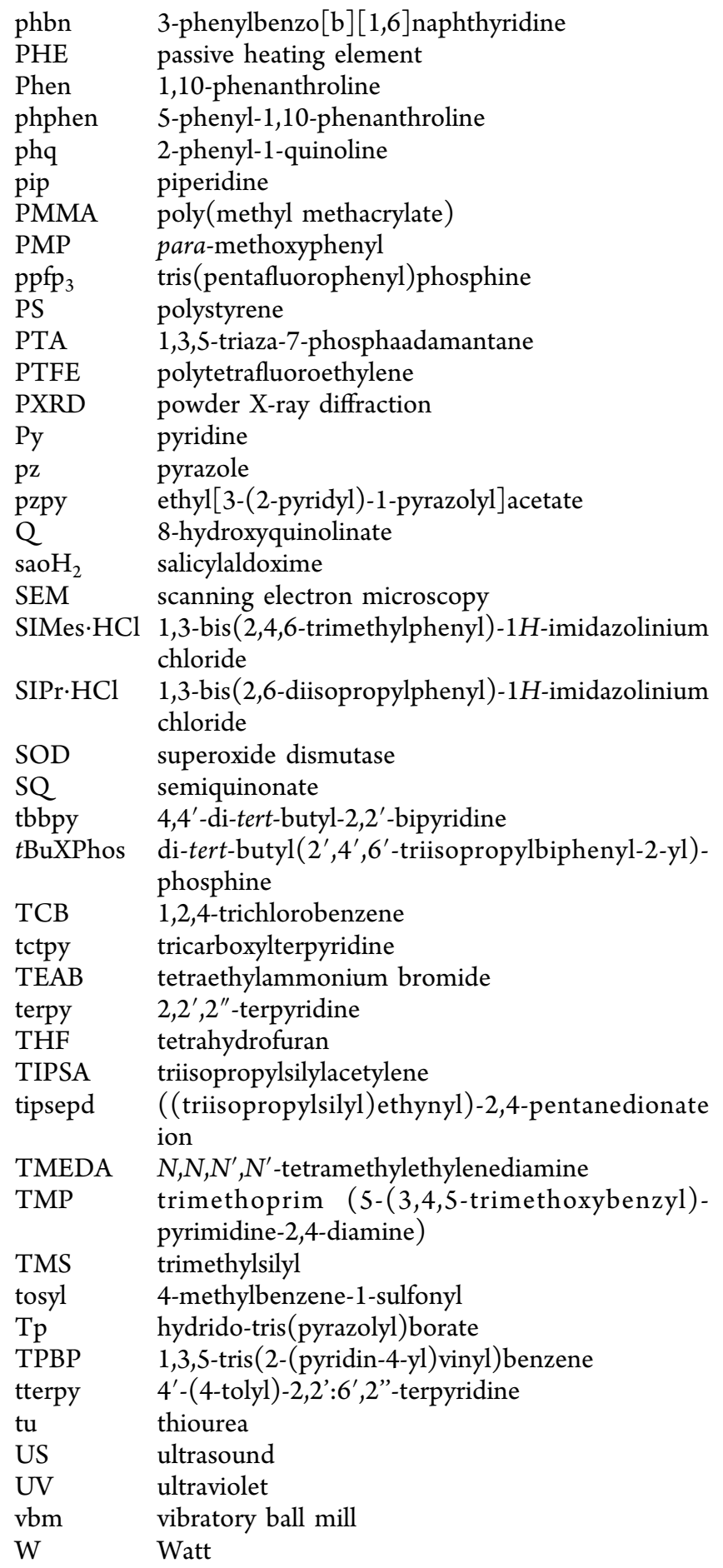

(1) Abe, T.; Miyazawa, A.; Kawanishi, Y.; Konno, H. MicrowaveAssisted Synthesis of Metal Complexes. Mini-Rev. Org. Chem. 2011, 8, 315-333.

(2) Rodríguez, A.; García-Vázquez, J. A. The Use Of Sacrificial Anodes For The Electrochemical Synthesis Of Metallic Complexes. Coord. Chem. Rev. 2015, 303, 42-85.

(3) Hoffmann, N. Photochemical Reactions as Key Steps in Organic Synthesis. Chem. Rev. 2008, 108, 1052-1103.

(4) Tan, D.; Friščić, T. Mechanochemistry for Organic Chemists: An Update. Eur. J. Org. Chem. 2018, 2018, 18-33.

(5) Ley, S. V.; Fitzpatrick, D. E.; Ingham, R. J.; Myers, R. M. Organic Synthesis: March Of The Machines. Angew. Chem., Int. Ed. 2015, 54, 3449-3464.

NHC N-heterocyclic carbene

NIS $\quad N$-iodosuccinimide

NMI N-methylimidazole

NMR nuclear magnetic resonance

nosyl 4-nitrobenzene-1-sulfonyl

OLED organic light-emitting diode

pbm planetary ball mill

PEG polyethylene glycol

PEPPSI pyridine-enhanced precatalyst preparation stabilization and initiation

pfm perfluorobutyramide 
(6) Bruijnincx, P. C. A.; Sadler, P. J. New Trends For Metal Complexes With Anticancer Activity. Curr. Opin. Chem. Biol. 2008, 12, 197-206.

(7) Zhang, C. X.; Lippard, S. J. New Metal Complexes As Potential Therapeutics. Curr. Opin. Chem. Biol. 2003, 7, 481-489.

(8) Meier-Menches, S. M.; Gerner, C.; Berger, W.; Hartinger, C. G.; Keppler, B. K. Structure-Activity Relationships For Ruthenium And Osmium Anticancer Agents - Towards Clinical Development. Chem. Soc. Rev. 2018, 47, 909-928.

(9) Chen, Z.-F.; Orvig, C.; Liang, H. Multi-Target Metal-Based Anticancer Agents. Curr. Top. Med. Chem. 2017, 17, 3131-3145.

(10) Patanjali, P.; Kumar, R.; Sourabh; Kumar, A.; Chaudhary, P.; Singh, R. Reviewing Gold(III) Complexes As Effective Biological Operators. Main Group Chem. 2018, 17, 35-52.

(11) Mital, M.; Ziora, Z. Biological Applications Of $\mathrm{Ru}(\mathrm{II})$ Polypyridyl Complexes. Coord. Chem. Rev. 2018, 375, 434-458.

(12) Hanif, M.; Hartinger, C. G. Anticancer Metallodrugs: Where Is The Next Cisplatin? Future Med. Chem. 2018, 10, 615-617.

(13) Popolin, C. P.; Cominetti, M. R. A Review of Ruthenium Complexes Activities on Breast Cancer Cells. Mini-Rev. Med. Chem. 2017, 17, 1435-1441.

(14) Leon, I. E.; Cadavid-Vargas, J. F.; Di Virgilio, A. L.; Etcheverry, S. B. Vanadium, Ruthenium and Copper Compounds: A New Class of Nonplatinum Metallodrugs with Anticancer Activity. Curr. Med. Chem. 2017, 24, 112-148.

(15) Ali, B.; Iqbal, M. A. Coordination Complexes of Manganese and Their Biomedical Applications. ChemistrySelect 2017, 2, 15861604.

(16) Jurowska, A.; Jurowski, K.; Szklarzewicz, J.; Buszewski, B.; Kalenik, T.; Piekoszewski, W. Molybdenum Metallopharmaceuticals Candidate Compounds - The "Renaissance" of Molybdenum Metallodrugs? Curr. Med. Chem. 2016, 23, 3322-3342.

(17) Bodio, E.; Le Gendre, P.; Denat, F.; Goze, C. Development Of Trackable Anticancer Agents Based On Metal Complexes. Adv. Inorg. Chem. 2016, 68, 253-299.

(18) James, S. L.; Adams, C. J.; Bolm, C.; Braga, D.; Collier, P.; Friščić, T.; Grepioni, F.; Harris, K. D. M.; Hyett, G.; Jones, W.; Krebs, A.; Mack, J.; Maini, L.; Orpen, A. G.; Parkin, I. P.; Shearouse, W. C.; Steed, J. W.; Waddell, D. C. Mechanochemistry: Opportunities For New And Cleaner Synthesis. Chem. Soc. Rev. 2012, 41, 413-447.

(19) Bowmaker, G. A. Solvent-Assisted Mechanochemistry. Chem. Commun. 2013, 49, 334-348.

(20) Hernández, J. G.; Bolm, C. Altering Product Selectivity by Mechanochemistry. J. Org. Chem. 2017, 82, 4007-4019.

(21) Métro, T.-X.; Bonnamour, J.; Reidon, T.; Duprez, A.; Sarpoulet, J.; Martinez, J.; Lamaty, F. Comprehensive Study of the OrganicSolvent-Free CDI-Mediated Acylation of Various Nucleophiles by Mechanochemistry. Chem. - Eur. J. 2015, 21, 12787-12796.

(22) Métro, T.-X.; Bonnamour, J.; Reidon, T.; Sarpoulet, J.; Martinez, J.; Lamaty, F. Mechanosynthesis Of Amides In The Total Absence Of Organic Solvent From Reaction To Product Recovery. Chem. Commun. 2012, 48, 11781-11783.

(23) Kaupp, G. Waste-Free Large-Scale Syntheses Without Auxiliaries For Sustainable Production Omitting Purifying Workup. CrystEngComm 2006, 8, 794-804.

(24) Kaupp, G.; Schmeyers, J.; Naimi-Jamal, M. R.; Zoz, H.; Ren, H. Reactive Milling With The Simoloyer ${ }^{\circledR}$ : Environmentally Benign Quantitative Reactions Without Solvents And Wastes. Chem. Eng. Sci. 2002, 57, 763-765.

(25) https://www.equipnet.com/mp_data/media/2008951251_ 185006 1.pdf (accessed May 27, 2018).

(26) Crawford, D. E. Extrusion - Back To The Future: Using An Established Technique To Reform Automated Chemical Synthesis. Beilstein J. Org. Chem. 2017, 13, 65-75.

(27) Loupy, A. Microwaves in Organic Synthesis. In Microwaves in Organic Synthesis; Wiley-VCH, 2006.

(28) Rathi, A. K.; Gawande, M. B.; Zboril, R.; Varma, R. S. Microwave-Assisted Synthesis - Catalytic Applications In Aqueous Media. Coord. Chem. Rev. 2015, 291, 68-94.
(29) de la Hoz, A.; Diaz-Ortiz, A.; Moreno, A. Activation Of Organic Reactions By Microwaves. Adv. Org. Synth. 2005, 1, 119-171.

(30) Loupy, A.; Perreux, L.; Liagre, M.; Burle, K.; Moneuse, M. Reactivity And Selectivity Under Microwaves In Organic Chemistry. Relation With Medium Effects And Reaction Mechanisms. Pure Appl. Chem. 2001, 73, 161-166.

(31) Loupy, A.; Petit, A.; Hamelin, J.; Texier-Boullet, F.; Jacquault, P.; Mathe, D. New Solvent-Free Organic Synthesis Using Focused Microwaves. Synthesis 1998, 1998, 1213-1234.

(32) Obermayer, D.; Gutmann, B.; Kappe, O. C. Microwave Chemistry in Silicon Carbide Reaction Vials: Separating Thermal from Nonthermal Effects. Angew. Chem., Int. Ed. 2009, 48, 83218324.

(33) de la Hoz, A.; Díaz-Ortiz, Á.; Moreno, A. Microwaves In Organic Synthesis. Thermal And Non-Thermal Microwave Effects. Chem. Soc. Rev. 2005, 34, 164-178.

(34) Gutmann, B.; Schwan, A. M.; Reichart, B.; Gspan, C.; Hofer, F.; Kappe, O. C. Activation and Deactivation of a Chemical Transformation by an Electromagnetic Field: Evidence for Specific Microwave Effects in the Formation of Grignard Reagents. Angew. Chem., Int. Ed. 2011, 50, 7636-7640.

(35) Suslick, K. S. Sonochemistry. Science 1990, 247, 1439-1445.

(36) Suslick, K. S.; Flannigan, D. J. Inside a Collapsing Bubble: Sonoluminescence and the Conditions During Cavitation. Annu. Rev. Phys. Chem. 2008, 59, 659-683.

(37) Flint, E. B.; Suslick, K. S. The Temperature of Cavitation. Science 1991, 253, 1397-1399.

(38) Grieser, F. Sonochemistry and the acoustic bubble; Elsevier: Amsterdam; Boston, 2015; p xvi, 282 pages.

(39) Mason, T. J. Practical sonochemistry: user's guide to applications in chemistry and chemical engineering; E. Horwood: New York, 1991; p 186.

(40) Mason, T. J.; Peters, D. Practical Sonochemistry: Power Ultrasound Uses and Applications; Elsevier Science: 2002.

(41) Mason, T. J. Ultrasound In Synthetic Organic Chemistry. Chem. Soc. Rev. 1997, 26, 443-451.

(42) Glusac, K. What Has Light Ever Done For Chemistry? Nat. Chem. 2016, 8, 734.

(43) Turro, N. J.; Ramamurthy, V.; Scaiano, J. C. Modern Molecular Photochemistry Of Organic Molecules; University Science Books: Sausalito, Calif., 2010; p xxxiii, 1084 p.

(44) Turro, N. J.; Ramamurthy, V.; Scaiano, J. C. Principles Of Molecular Photochemistry: An Introduction; University Science Books: Sausalito, Calif., 2009; p xxi, 495 p.

(45) Klán, P.; Wirz, J. Photochemistry Of Organic Compounds: From Concepts To Practice; Wiley: Chichester, West Sussex, U.K., 2009; p xviii, $563 \mathrm{p}$.

(46) Braun, A. M.; Maurette, M.-T. r. s.; Oliveros, E. Photochemical Technology; Wiley: Chichester, West Sussex, England; New York, 1991; p xx, 559 p.

(47) http://www.edisontechcenter.org/MercuryVaporLamps.html (accessed April 27, 2018).

(48) Gutmann, B.; Cantillo, D.; Kappe, C. O. Continuous-Flow Technology-A Tool for the Safe Manufacturing of Active Pharmaceutical Ingredients. Angew. Chem., Int. Ed. 2015, 54, 66886728.

(49) Damm, M.; Glasnov, T. N.; Kappe, C. O. Translating HighTemperature Microwave Chemistry to Scalable Continuous Flow Processes. Org. Process Res. Dev. 2010, 14, 215-224.

(50) Movsisyan, M.; Delbeke, E. I. P.; Berton, J. K. E. T.; Battilocchio, C.; Ley, S. V.; Stevens, C. V. Taming Hazardous Chemistry By Continuous Flow Technology. Chem. Soc. Rev. 2016, 45, 4892-4928.

(51) Fukuyama, T.; Chiba, H.; Kuroda, H.; Takigawa, T.; Kayano, A.; Tagami, K. Application of Continuous Flow for DIBAL-H Reduction and $n$-BuLi Mediated Coupling Reaction in the Synthesis of Eribulin Mesylate. Org. Process Res. Dev. 2016, 20, 503-509. 
(52) Amara, Z.; Streng, E. S.; Skilton, R. A.; Jin, J.; George, M. W.; Poliakoff, M. Automated Serendipity with Self-Optimizing Continuous-Flow Reactors. Eur. J. Org. Chem. 2015, 2015, 6141-6145.

(53) Hsieh, H.-W.; Coley, C. W.; Baumgartner, L. M.; Jensen, K. F.; Robinson, R. I. Photoredox Iridium-Nickel Dual-Catalyzed Decarboxylative Arylation Cross-Coupling: From Batch to Continuous Flow via Self-Optimizing Segmented Flow Reactor. Org. Process Res. Dev. 2018, 22, 542-550.

(54) Brzozowski, M.; O’Brien, M.; Ley, S. V.; Polyzos, A. Flow Chemistry: Intelligent Processing of Gas-Liquid Transformations Using a Tube-in-Tube Reactor. Acc. Chem. Res. 2015, 48, 349-362.

(55) Britton, J.; Jamison, T. F. The Assembly And Use Of Continuous Flow Systems For Chemical Synthesis. Nat. Protoc. 2017, $12,2423-2446$

(56) Skilton, R. A.; Bourne, R. A.; Amara, Z.; Horvath, R.; Jin, J.; Scully, M. J.; Streng, E.; Tang, S. L. Y.; Summers, P. A.; Wang, J.; Pérez, E.; Asfaw, N.; Aydos, G. L. P.; Dupont, J.; Comak, G.; George, M. W.; Poliakoff, M. Remote-Controlled Experiments With Cloud Chemistry. Nat. Chem. 2015, 7, 1-5.

(57) Dabirmanesh, Q.; Roberts, R. M. G. The Application Of Microwave Dielectric Heating To The Synthesis Of Arene-Metal Complexes. Synthesis of $\left[(\eta\right.$-arene $\left.)(\mathrm{CO})_{3} \mathrm{Mn}\right] \mathrm{PF}_{6}$ Complexes And $[(\eta$-arene $)(\eta$-cyclopentadienyl $) \mathrm{Fe}]\left[\mathrm{PF}_{6}\right]$ Complexes With Triphenylphosphine, Tert-Butylbenzenes And A Sterically Hindered Phenol As Arene Ligands. J. Organomet. Chem. 1997, 542, 99-103.

(58) Knochel, P.; Gavryushin, A.; Krasovskiy, A.; Leuser, H., 9.03 Magnesium. In Comprehensive Organometallic Chemistry III; Mingos, D. M. P., Crabtree, R. H., Eds.; Elsevier: Oxford, 2007; pp 31-79.

(59) Cravotto, G.; Procopio, A.; Oliverio, M.; Orio, L.; Carnaroglio, D. Simple Sonochemical Protocols For Fast And Reproducible Grignard Reactions. Green Chem. 2011, 13, 2806-2809.

(60) Harrowfield, J. M.; Hart, R. J.; Whitaker, C. R. Magnesium And Aromatics: Mechanically-Induced Grignard And Mcmurry Reactions. Aust. J. Chem. 2001, 54, 423-425.

(61) van de Kruijs, B. H. P.; Dressen, M. H. C. L.; Meuldijk, J.; Vekemans, J. A. J. M.; Hulshof, L. A. Microwave-Induced Electrostatic Etching: Generation Of Highly Reactive Magnesium For Application In Grignard Reagent Formation. Org. Biomol. Chem. 2010, 8, 16881694.

(62) Qian, Z.; Baxendale, I. R.; Ley, S. V. A Continuous Flow Process Using a Sequence of Microreactors with In-line IR Analysis for the Preparation of N,N-Diethyl-4-(3-fluorophenylpiperidin-4ylidenemethyl)benzamide as a Potent and Highly Selective $\delta$-Opioid Receptor Agonist. Chem. - Eur. J. 2010, 16, 12342-12348.

(63) Tricotet, T.; O'Shea, D. F. Automated Generation and Reactions of 3-Hydroxymethylindoles in Continuous-Flow Microreactors. Chem. - Eur. J. 2010, 16, 6678-6686.

(64) Deng, Q.; Shen, R.; Zhao, Z.; Yan, M.; Zhang, L. The Continuous Flow Synthesis Of 2,4,5-Trifluorobenzoic Acid Via Sequential Grignard Exchange And Carboxylation Reactions Using Microreactors. Chem. Eng. J. 2015, 262, 1168-1174.

(65) Brodmann, T.; Koos, P.; Metzger, A.; Knochel, P.; Ley, S. V. Continuous Preparation of Arylmagnesium Reagents in Flow with Inline IR Monitoring. Org. Process Res. Dev. 2012, 16, 1102-1113.

(66) Deng, Q.; Shen, R.; Ding, R.; Zhang, L. Generation of EthynylGrignard Reagent in a Falling Film Microreactor: An Expeditious Flow Synthesis of Propargylic Alcohols and Analogues. Adv. Synth. Catal. 2014, 356, 2931-2936.

(67) Goldbach, M.; Danieli, E.; Perlo, J.; Kaptein, B.; Litvinov, V. M.; Blümich, B.; Casanova, F.; Duchateau, A. L. L. Preparation Of Grignard Reagents From Magnesium Metal Under Continuous Flow Conditions And On-Line Monitoring By NMR Spectroscopy. Tetrahedron Lett. 2016, 57, 122-125.

(68) Kopach, M. E.; Cole, K. P.; Pollock, P. M.; Johnson, M. D.; Braden, T. M.; Webster, L. P.; McClary Groh, J.; McFarland, A. D.; Schafer, J. P.; Adler, J. J.; Rosemeyer, M. Flow Grignard and Lithiation: Screening Tools and Development of Continuous Processes for a Benzyl Alcohol Starting Material. Org. Process Res. Dev. 2016, 20, 1581-1592.
(69) Degennaro, L.; Maggiulli, D.; Carlucci, C.; Fanelli, F.; Romanazzi, G.; Luisi, R. A Direct And Sustainable Synthesis Of Tertiary Butyl Esters Enabled By Flow Microreactors. Chem. Commun. 2016, 52, 9554-9557.

(70) Degennaro, L.; Carlucci, C.; Angelis, S. D.; Luisi, R. Flow Technology For Organometallic-Mediated Synthesis. J. Flow Chem. 2016, 6, 136-166.

(71) Murray, P. R. D.; Browne, D. L.; Pastre, J. C.; Butters, C.; Guthrie, D.; Ley, S. V. Continuous Flow-Processing of Organometallic Reagents Using an Advanced Peristaltic Pumping System and the Telescoped Flow Synthesis of $(E / Z)$-Tamoxifen. Org. Process Res. Dev. 2013, 17, 1192-1208.

(72) Nagaki, A.; Ichinari, D.; Yoshida, J.-i. Reactions Of Organolithiums With Dialkyl Oxalates. A Flow Microreactor Approach To Synthesis Of Functionalized $\alpha$-Keto Esters. Chem. Commun. 2013, 49, 3242-3244.

(73) Nagaki, A.; Tokuoka, S.; Yoshida, J.-i. Flash Generation of $\alpha$ (Trifluoromethyl)Vinyllithium And Application To Continuous Flow Three-Component Synthesis Of A-Trifluoromethylamides. Chem. Commun. 2014, 50, 15079-15081.

(74) Nagaki, A.; Uesugi, Y.; Kim, H.; Yoshida, J.-i. Synthesis of Functionalized Aryl Fluorides Using Organolithium Reagents in Flow Microreactors. Chem. - Asian J. 2013, 8, 705-708.

(75) Kim, H.; Nagaki, A.; Yoshida, J.-i. A Flow-Microreactor Approach To Protecting-Group-Free Synthesis Using Organolithium Compounds. Nat. Commun. 2011, 2, 264-269.

(76) Degennaro, L.; Fanelli, F.; Giovine, A.; Luisi, R. External Trapping of Halomethyllithium Enabled by Flow Microreactors. Adv. Synth. Catal. 2015, 357, 21-27.

(77) Gupta, K. C.; Sutar, A. K. Catalytic Activities Of Schiff Base Transition Metal Complexes. Coord. Chem. Rev. 2008, 252, 14201450.

(78) Abu-Dief, A. M.; Mohamed, I. M. A. A Review On Versatile Applications Of Transition Metal Complexes Incorporating Schiff Bases. Beni-SUEF Univ. J. Appl. Sci. 2015, 4, 119-133.

(79) Makhaev, V. D.; Petrova, L. A.; Bravaya, N. M.; Faingol'd, E. E.; Mukhina, E. V.; Panin, A. N.; Gagieva, S. C.; Tuskaev, V. A.; Bulychev, B. M. Mechanochemical Synthesis Of Zirconium And Hafnium Phenoxyimine Complexes $\mathrm{L}_{2} \mathrm{MCl}_{2}(\mathrm{~L}=\mathrm{N}$-(3,5-di-tertbutylsalicylidene)-2,3,5,6-tetrafluoroanilinate anion) and Their Catalytic Properties In Ethylene Polymerization. Russ. Chem. Bull. 2014, 63, 1533-1538.

(80) Rightmire, N. R.; Hanusa, T. P.; Rheingold, A. L. Mechanochemical Synthesis of $\left[1,3-\left(\mathrm{SiMe}_{3}\right)_{2} \mathrm{C}_{3} \mathrm{H}_{3}\right]_{3}(\mathrm{Al}, \mathrm{Sc})$, a BaseFree Tris(allyl)aluminum Complex and Its Scandium Analogue. Organometallics 2014, 33, 5952-5955.

(81) Boyde, N. C.; Rightmire, N. R.; Bierschenk, E. J.; Steelman, G. W.; Hanusa, T. P.; Brennessel, W. W. Reaction Environment And Ligand Lability In Group $4 \mathrm{Cp}_{2} \mathrm{MXY}(\mathrm{X}, \mathrm{Y}=\mathrm{Cl}$, OtBu) complexes. Dalton Trans 2016, 45, 18635-18642.

(82) Makhaev, V. D.; Petrova, L. A. Mechanochemical Synthesis Of Vanadium(III) $\beta$-Diketonates. Russ. J. Gen. Chem. 2017, 87, 11051109.

(83) Alias, M. F.; Jaafer, F. S.; Sadiq, A. S. Synthesis Mixed Ligands Complexes Of Some Light Metals By Microwave Assisted Using 2Acetamide Benzothiazole And 1,10-Phenanthroline. Int. J. Pharm. 2015, 5, 451-458.

(84) Thaker, B. T.; Barvalia, R. S. Microwave Assisted Synthesis And Characterization Of Unsymmetrical Tetradentate Schiff Base Complexes Of VO(IV) And Moo(V). Spectrochim. Acta, Part A 2011, 84, 51-61.

(85) Merzougui, M.; Ouari, K.; Weiss, J. Ultrasound Assisted Synthesis, Characterization And Electrochemical Study Of A Tetradentate Oxovanadium Diazomethine Complex. J. Mol. Struct. 2016, 1120, 239-244.

(86) McGarrigle, E. M.; Gilheany, D. G. Chromium- and Manganese-Salen Promoted Epoxidation of Alkenes. Chem. Rev. 2005, 105, 1563-1602. 
(87) Borisov, A. P.; Petrova, L. A.; Karpova, T. P.; Makhaev, V. D. Solid-State Mechanochemical Synthesis Of Tris(4-Imino-2Pentanonato)Chromium, $\mathrm{Cr}\left[\mathrm{CH}_{3} \mathrm{C}(\mathrm{NH}) \mathrm{CHC}(\mathrm{O}) \mathrm{CH}_{3}\right]_{3}$. Russ. Chem. Bull. 1994, 43, 2107-2109.

(88) Uzarevic, K.; Rubcic, M.; Radic, M.; Puskaric, A.; Cindric, M. Mechanosensitive Metal-Ligand Bonds In The Design Of New Coordination Compounds. CrystEngComm 2011, 13, 4314-4323.

(89) Užarević, K.; Rubčić, M.; Bilovic, I.; Kokan, Z.; MatkovićCalogović, D.; Cindrić, M. Concomitant Conformational Polymorphism: Mechanochemical Reactivity and Phase Relationships in the (Methanol)cis-dioxo( $\mathrm{N}$-salicylidene-2-amino-3-hydroxypyridine)molybdenum(VI) Trimorph. Cryst. Growth Des. 2009, 9, 5327-5333.

(90) Cindric, M.; Pavlovic, G.; Katava, R.; Agustin, D. Towards A Global Greener Process: From Solvent-Less Synthesis Of Molybdenum(Vi) ONO Schiff Base Complexes To Catalyzed Olefin Epoxidation Under Organic-Solvent-Free Conditions. New J. Chem. 2017, 41, 594-602.

(91) Baghurst, D. R.; Cooper, S. R.; Greene, D. L.; Mingos, D. M. P.; Reynolds, S. M. Application Of Microwave Dielectric Loss Heating Effects For The Rapid And Convenient Synthesis Of Coordination Compounds. Polyhedron 1990, 9, 893-895.

(92) VanAtta, S. L.; Duclos, B. A.; Green, D. B. Microwave-Assisted Synthesis of Group 6 (Cr, Mo, W) Zerovalent Organometallic Carbonyl Compounds. Organometallics 2000, 19, 2397-2399.

(93) Ardon, M.; Hogarth, G.; Oscroft, D. T. W. Organometallic Chemistry In A Conventional Microwave Oven: The Facile Synthesis Of Group 6 Carbonyl Complexes. J. Organomet. Chem. 2004, 689, 2429-2435.

(94) Birdwhistell, K. R.; Schulz, B. E.; Dizon, P. M. Rapid Synthesis Of Group VI Carbonyl Complexes By Coupling Borohydride Catalysis And Microwave Heating. Inorg. Chem. Commun. 2012, 26, 69-71.

(95) Jain, R. K.; Mishra, A. P. Microwave Synthesis And Spectral, Thermal And Antimicrobial Activities Of Some Novel Transition Metal Complexes With Tridentate Schiff Base Ligands. J. Serb. Chem. Soc. 2012, 77, 1013-1029.

(96) Mishra, A. P.; Jain, R. K. Microwave Synthesis, Spectral, Thermal and Antimicrobial Activities of Some Transition Metal Complexes Involving 2-Amino-6-nitrobenzothiazole Moiety. Proc. Natl. Acad. Sci., India, Sect. A 2013, 83, 213-223.

(97) Jain, R. K.; Mishra, A. P. Microwave Synthesis, Spectral, Thermal, 3D Molecular Modeling Analysis And Antimicrobial Activities Of Some Transition Metal Complexes Of Schiff Bases Derived From 5-Bromosalicylaldehyde. J. Saudi Chem. Soc. 2016, 20, 127-137.

(98) Jain, R. K.; Mishra, A. P.; Mishra, D. K.; Gupta, S. K. Microwave Synthesis, Spectral, Thermal And Electrical Properties Of Some Metal Complexes Involving 5-Bromosalicylaldehyde. E-J. Chem. 2012, 9, 1721-1727.

(99) Mishra, A. P.; Jain, R. K. Conventional And Microwave Synthesis, Spectral, Thermal And Antimicrobial Studies Of Some Transition Metal Complexes Containing 2-Amino-5-Methylthiazole Moiety. J. Saudi Chem. Soc. 2014, 18, 814-824.

(100) Mishra, A. P.; Jain, R. K. Microwave Synthesis, Spectroscopic, Thermal And Biological Significance Of Some Transition Metal Complexes Containing Heterocyclic Ligands. Transition Met. Chem. 2010, 2, 51-61.

(101) Fahmi, N.; Shrivastava, S.; Meena, R.; Joshi, S. C.; Singh, R. V. Microwave Assisted Synthesis, Spectroscopic Characterization And Biological Aspects Of Some New Chromium(III) Complexes Derived From $\mathrm{N}^{\wedge} \mathrm{O}$ Donor Schiff Bases. New J. Chem. 2013, 37, 1445-1453. (102) Praveen Kumar, S.; Suresh, R.; Giribabu, K.; Manigandan, R.; Munusamy, S.; Muthamizh, S.; Narayanan, V. Synthesis And Characterization Of Chromium(III) Schiff Base Complexes: Antimicrobial Activity And Its Electrocatalytic Sensing Ability Of Catechol. Spectrochim. Acta, Part A 2015, 139, 431-441.

(103) Whittaker, A. G.; Mingos, D. M. P. Synthetic Reactions Using Metal Powders Under Microwave Irradiation. J. Chem. Soc., Dalton Trans. 2002, 3967-3970.
(104) Lee, Y. T.; Choi, S. Y.; Lee, S. I.; Chung, Y. K.; Kang, T. J. Microwave-Assisted Synthesis Of $\left(\mathrm{H}^{6}\right.$-Arene)Tricarbonylchromium Complexes. Tetrahedron Lett. 2006, 47, 6569-6572.

(105) Amarante, T.; Almeida Paz, F.; Gago, S.; Gonçalves, I.; Pillinger, M.; Rodrigues, A.; Abrantes, M. Microwave-Assisted Synthesis and Crystal Structure of Oxo(diperoxo)(4,4'-di-tert-butyl2,2'-bipyridine)-molybdenum(VI). Molecules 2009, 14, 3610-3620.

(106) Ardon, M.; Hayes, P. D.; Hogarth, G. Microwave-Assisted Reflux in Organometallic Chemistry: Synthesis and Structural Determination of Molybdenum Carbonyl Complexes. An Intermediate-Level Organometallic-Inorganic Experiment. J. Chem. Educ. 2002, 79, 1249-1251.

(107) Coelho, A.; Almeida Paz, F.; Klinowski, J.; Pillinger, M.; Gonçalves, I. Microwave Assisted Synthesis of Molybdenum and Tungsten Tetracarbonyl Complexes with a Pyrazolylpyridine Ligand. Crystal structure of cis-[Mo(CO) ${ }_{4}\{$ ethyl[3-(2-pyridyl)-1-pyrazolyl] acetate\}]. Molecules 2006, 11, 940-952.

(108) Barnard, T. M.; Leadbeater, N. E. Real-Time Monitoring Of Microwave-Promoted Organometallic Ligand-Substitution Reactions Using In Situ Raman Spectroscopy. Chem. Commun. 2006, 36153616.

(109) Artillo, A.; Sala, G. D.; De Santis, M.; Llordes, A.; Ricart, S.; Spinella, A. Preparation of organometallic uracil-analogue Fischer carbene complexes: Comparative study of conventional heating vs microwave irradiation. J. Organomet. Chem. 2007, 692, 1277-1284.

(110) Kostermans, G. B. M.; Bobeldijk, M.; Kwakman, P. J.; de Wolf, W. H.; Bickelhaupt, F. Photochemical Synthesis Of Arenetricarbonylchromium(0) Complexes: Scope And Limitations. J. Organomet. Chem. 1989, 363, 291-296.

(111) Bengali, A. A.; Charlton, S. B. Displacement of the Benzene Solvent Molecule from $\mathrm{Cr}(\mathrm{CO})_{5}$ (benzene) by Piperidine: A Laser Flash Photolysis Experiment. J. Chem. Educ. 2000, 77, 1348-1351.

(112) Deckelmann, K.; Werner, H. Photochemische Darstellung von Hexaalkylborazol-und Aromaten-chromtricarbonylen. Helv. Chim. Acta 1970, 53, 139-141.

(113) McNeese, T. J.; Ezbiansky, K. A. Photochemical Preparation and Reactivity of cis $\mathrm{Cr}(\mathrm{CO})_{4}\left(\mathrm{CH}_{3} \mathrm{CN}\right)_{2}$ : An Advanced Undergraduate Inorganic Laboratory Experiment. J. Chem. Educ. 1996, 73, $548-550$.

(114) King, J. L.; Molvinger, K.; Poliakoff, M. Organometallic Synthesis as a Continuous Process: The Synthesis and Isolation of $\mathrm{Cr}(\mathrm{CO})_{5}\left(\eta^{2}-\mathrm{C}_{3} \mathrm{H}_{6}\right)$ and $\left(\mathrm{C}_{5} \mathrm{R}_{5}\right) \mathrm{Mn}(\mathrm{CO})_{2}\left(\eta^{2}-\mathrm{C}_{3} \mathrm{H}_{6}\right)(\mathrm{R}=\mathrm{H}$ and $\mathrm{Me})$ from Superheated Liquid Propene. Organometallics 2000, 19, 50775082.

(115) Kocięcka, P.; Kochel, A.; Szymańska-Buzar, T. Photochemical Synthesis, Structure And Spectroscopic Properties Of [W$(\mathrm{CO})_{4}\left(\mathrm{C}_{5} \mathrm{H}_{10} \mathrm{~N}\right)_{2} \mathrm{CH}_{2}$ ]. Inorg. Chem. Commun. 2014, 45, 105-107.

(116) Cortés-Figueroa, J. E. An Experiment for the Inorganic Chemistry Laboratory: The Sunlight-Induced Photosynthesis of $\left(\eta^{2}\right.$ $\left.\mathrm{C}_{60}\right) \mathrm{M}(\mathrm{CO})_{5}$ Complexes $(\mathrm{M}=\mathrm{Mo}, \mathrm{W})$. J. Chem. Educ. 2003, 80, 799-800.

(117) Post, E. W. An Inorganic Laboratory Experiment Involving Photochemistry, Liquid Chromatography, And Infrared Spectroscopy. J. Chem. Educ. 1980, 57, 819-822.

(118) Manuta, D. M.; Lees, A. J. Preparation And Reaction Of 2,2'Bipyridinetetracarbonylmolybdenum(0): An Experiment In Organometallic Photochemistry. J. Chem. Educ. 1987, 64, 637-638.

(119) Sener, S.; Ercag, A. Photochemical Reactions Of Metal Carbonyls $\left[\mathrm{M}(\mathrm{CO})_{6}(\mathrm{M}=\mathrm{Cr}, \mathrm{Mo}, \mathrm{W}), \mathrm{Mn}(\mathrm{CO})_{3} \mathrm{Cp}\right]$ With 3[4Ethyl(Phenyl)Imino][1H-Indol-2-One] And 3[4-Butyl(Phenyl)Imino][1H-Indol-2-One]. Asian J. Chem. 2017, 29, 235-238.

(120) Lee, C. X.; Pedrick, E. A.; Leadbeater, N. E. Preparation of Arene Chromium Tricarbonyl Complexes Using Continuous-Flow Processing: $\left(\eta^{6}-\mathrm{C}_{6} \mathrm{H}_{5} \mathrm{CH}_{3}\right) \mathrm{Cr}(\mathrm{CO})_{3}$ as an Example. J. Flow Chem. 2012, 2, 115-117.

(121) Fernandez-Moreira, V.; Thorp-Greenwood, F. L.; Coogan, M. P. Application Of d6 Transition Metal Complexes In Fluorescence Cell Imaging. Chem. Commun. 2010, 46, 186-202. 
(122) Hernandez, J. G.; Macdonald, N. A. J.; Mottillo, C.; Butler, I. S.; Friščić, T. A Mechanochemical Strategy For Oxidative Addition: Remarkable Yields And Stereoselectivity In The Halogenation Of Organometallic $\operatorname{Re}(\mathrm{I})$ Complexes. Green Chem. 2014, 16, 1087-1092. (123) Hernandez, J. G.; Butler, I. S.; Friščić, T. Multi-Step And Multi-Component Organometallic Synthesis In One Pot Using Orthogonal Mechanochemical Reactions. Chem. Sci. 2014, 5, 35763582.

(124) Glavinovic, M.; Qi, F.; Katsenis, A. D.; Friščić, T.; Lumb, J.-P. Redox-Promoted Associative Assembly Of Metal-Organic Materials. Chem. Sci. 2016, 7, 707-712.

(125) Baghurst, D. R.; Mingos, D. M. P. Design And Application Of A Reflux Modification For The Synthesis Of Organometallic Compounds Using Microwave Dielectric Loss Heating Effects. J. Organomet. Chem. 1990, 384, C57-C60.

(126) Reed, C. R.; Feeney, C.; Merritt, M. A. Microwave Synthesis Of Dirhenium Paddlewheel Complexes. J. Coord. Chem. 2015, 68, 3449-3456.

(127) Causey, P. W.; Besanger, T. R.; Schaffer, P.; Valliant, J. F. Expedient Multi-Step Synthesis of Organometallic Complexes of Tc and Re in High Effective Specific Activity. A New Platform for the Production of Molecular Imaging and Therapy Agents. Inorg. Chem. 2008, 47, 8213-8221.

(128) Kunz, P. C.; Berghahn, M.; Brückmann, N. E.; Dickmeis, M.; Kettel, M.; Spingler, B. Functionalised Tris(pyrazolyl)methane Ligands and $\operatorname{Re}(\mathrm{CO})_{3}$ Complexes Thereof. Z. Anorg. Allg. Chem. 2009, 635, 471-478.

(129) Armstrong, A. F.; Valliant, J. F. Microwave-Assisted Synthesis of Tricarbonyl Rhenacarboranes: Steric and Electronic Effects on the $1,2 \rightarrow$ 1,7 Carborane Cage Isomerization. Inorg. Chem. 2007, 46, $2148-2158$.

(130) Causey, P. W.; Besanger, T. R.; Valliant, J. F. Synthesis and Screening of Mono- and Di-Aryl Technetium and Rhenium Metallocarboranes. A New Class of Probes for the Estrogen Receptor. J. Med. Chem. 2008, 51, 2833-2844.

(131) Bhojak, N.; Gudasaria, D. D.; Khiwani, N.; Jain, R. Microwave Assisted Synthesis Spectral and Antibacterial Investigations on Complexes of $\mathrm{Mn}$ (II) With Amide Containing Ligands. E-J. Chem. 2007, 4, 232-237.

(132) Xu, S. P.; Xu, G.; Pei, Y.; Zhu, H. L. Microwave Solid Phase Synthesis, Characterization, And Antimicrobial Activities Of One Mononuclear Manganese(II) Complex With 4-Chlorobenzoic Acid 4[3-(4-chlorophenyl)-3-hydroxyacryloyl]-3-hydroxyphenyl ester. Russ. J. Coord. Chem. 2014, 40, 63-67.

(133) Ali, P.; Ramakanth, P.; Meshram, J. Exploring Microwave Synthesis For Co-Ordination: Synthesis, Spectral Characterization And Comparative Study Of Transition Metal Complexes With Binuclear Core Derived From 4-amino-2,3-dimethyl-1-phenyl-3pyrazolin-5-one. J. Coord. Chem. 2010, 63, 323-329.

(134) Ahmed, M. F. A. Microwave Synthesis And Antibacterial Activity Of Some Heterocyclic Schiff's Base Metal Complexes Of First Transition Series. Res. J. Pharm., Biol. Chem. Sci. 2014, 5, 912-919.

(135) Pagadala, R.; Ali, P.; Meshram, J. S. Microwave Assisted Synthesis And Characterization Of $N, N^{\prime}$-Bis(Salicylaldehydo)Ethylenediimine Complexes of $\mathrm{Mn}(\mathrm{II}), \mathrm{Co}(\mathrm{II}), \mathrm{Ni}(\mathrm{II})$, and $\mathrm{Zn}(\mathrm{II})$. J. Coord. Chem. 2009, 62, 4009-4017.

(136) Zhang, S.-H.; Feng, C. Microwave-Assisted Synthesis, Crystal Structure And Fluorescence Of Novel Coordination Complexes With Schiff Base Ligands. J. Mol. Struct. 2010, 977, 62-66.

(137) Milios, C. J.; Vinslava, A.; Whittaker, A. G.; Parsons, S.; Wernsdorfer, W.; Christou, G.; Perlepes, S. P.; Brechin, E. K. Microwave-Assisted Synthesis of a Hexanuclear Mn(III) SingleMolecule Magnet. Inorg. Chem. 2006, 45, 5272-5274.

(138) Batool, M.; Martin, T. A.; Naser, M. A.; George, M. W.; Macgregor, S. A.; Mahon, M. F.; Whittlesey, M. K. Comparison Of The Photochemistry Of Organometallic N-Heterocyclic Carbene And Phosphine Complexes Of Manganese. Chem. Commun. 2011, 47, 11225-11227.
(139) Batool, M.; Martin, T. A.; Algarra, A. G.; George, M. W.; Macgregor, S. A.; Mahon, M. F.; Whittlesey, M. K. Photochemistry of $\mathrm{Cp}^{\prime} \mathrm{Mn}(\mathrm{CO})_{2}(\mathrm{NHC})\left(\mathrm{Cp}^{\prime}=\eta^{5}-\mathrm{C}_{5} \mathrm{H}_{4} \mathrm{Me}\right)$ Species: Synthesis, TimeResolved IR Spectroscopy, and DFT Calculations. Organometallics 2012, 31, 4971-4979.

(140) Zhuang, J. M.; Sutton, D. Photolysis of ( $\eta^{5}$-pentamethylcyclopentadienyl)rhenium tricarbonyl With Alkenes. Synthesis of $\left(\eta^{5}\right.$ $\left.\mathrm{C}_{5} \mathrm{Me}_{5}\right) \operatorname{Re}(\mathrm{CO})_{2}$ (alkene) Complexes And Carbon-Hydrogen Activation To Give Allyl Hydrido Derivatives. Organometallics 1991, 10, $1516-1527$

(141) Plietker, B., Ed. Iron Catalysis in Organic Chemistry Reactions and Applications; Wiley-VCH Verlag GmbH \& Co. KGaA: 2008; p 279.

(142) van Rijt, S. H.; Sadler, P. J. Current Applications And Future Potential For Bioinorganic Chemistry In The Development Of Anticancer Drugs. Drug Discovery Today 2009, 14, 1089-1097.

(143) Younus, H. A.; Su, W.; Ahmad, N.; Chen, S.; Verpoort, F. Ruthenium Pincer Complexes: Synthesis and Catalytic Applications. Adv. Synth. Catal. 2015, 357, 283-330.

(144) Colacino, E.; Martinez, J.; Lamaty, F. Preparation of NHCRuthenium Complexes And Their Catalytic Activity In Metathesis Reaction. Coord. Chem. Rev. 2007, 251, 726-764.

(145) Scholl, M.; Ding, S.; Lee, C. W.; Grubbs, R. H. Synthesis and Activity of a New Generation of Ruthenium-Based Olefin Metathesis Catalysts Coordinated with 1,3-Dimesityl-4,5-dihydroimidazol-2ylidene Ligands. Org. Lett. 1999, 1, 953-956.

(146) Makhaev, V. D.; Borisov, A. P.; Petrova, L. A. Solid-State Mechanochemical Synthesis Of Ferrocene. J. Organomet. Chem. 1999, 590, 222-226.

(147) Braga, D.; Maini, L.; Polito, M.; Mirolo, L.; Grepioni, F. Mechanochemical Assembly Of Hydrogen Bonded Organic-Organometallic Solid Compounds. Chem. Commun. 2002, 2960-2961.

(148) Braga, D.; Maini, L.; Polito, M.; Mirolo, L.; Grepioni, F. Assembly of Hybrid Organic-Organometallic Materials through Mechanochemical Acid-Base Reactions. Chem. - Eur. J. 2003, 9, 4362-4370.

(149) Braga, D.; Giaffreda, S. L.; Grepioni, F.; Palladino, G.; Polito, M. Mechanochemical Assembly Of Hybrid Organic-Organometallic Materials. Solid-Solid Reactions Of 1,1'-di-pyridyl-ferrocene With Organic Acids. New J. Chem. 2008, 32, 820-828.

(150) Braga, D.; Giaffreda, S. L.; Grepioni, F. Solid-State Preparation Of Hybrid Organometallic-Organic Macrocyclic Adducts With Long Chain Dicarboxylic Acids. Chem. Commun. 2006, 3877-3879.

(151) Braga, D.; D’Addari, D.; Polito, M.; Grepioni, F. Mechanically Induced Expeditious and Selective Preparation of Disubstituted Pyridine/Pyrimidine Ferrocenyl Complexes. Organometallics 2004, 23, 2810-2812.

(152) Imrie, C.; Nyamori, V. O.; Gerber, T. I. A. Solvent-Free Synthesis Of Ferrocenylimines. J. Organomet. Chem. 2004, 689, $1617-1622$

(153) Imrie, C.; Kleyi, P.; Nyamori, V. O.; Gerber, T. I. A.; Levendis, D. C.; Look, J. Further Solvent-Free Reactions Of Ferrocenylaldehydes: Synthesis Of 1,1'-Ferrocenyldiimines and Ferrocenylacrylonitriles. J. Organomet. Chem. 2007, 692, 3443-3453.

(154) Kleyi, P. E.; McCleland, C. W.; Gerber, T. I. A. Solvent-Free Synthesis Of Bisferrocenylimines And Their Rhodium(I) Complexes. Polyhedron 2010, 29, 1095-1101.

(155) Ombaka, L. M.; Ndungu, P. G.; Omondi, B.; Nyamori, V. O. Mechanochemical Synthesis And Spectroscopic Properties Of $1,1^{\prime}$ Ferrocenyldiacrylonitriles: The Effect Of Para-Substituents. J. Coord. Chem. 2014, 67, 1905-1922.

(156) Nieto, D.; Bruna, S.; Montero-Campillo, M. M.; Perles, J.; Gonzalez-Vadillo, A. M. a.; Mendez, J.; Mo, O.; Cuadrado, I. Mechanochemical And Silica Gel-Mediated Formation Of Highly Electron-Poor 1-Cyanocarbonylferrocene. Chem. Commun. 2013, 49, 9785-9787.

(157) Borisov, A. P.; Makhaev, V. D.; Usyatinskii, A. Y.; Bregadze, V. I. Solid-State Mechanochemical Synthesis Of cobalt(III), iron(III), 
and chromium(III) Bisdicarbollyl Complexes. Russ. Chem. Bull. 1993, 42, 1637-1639.

(158) Paneque, A.; Reguera, E.; Fernández-Bertrán, J.; Yee-Madeira, H. Mechanochemical Reactions Of Fluorides With Hemin. J. Fluorine Chem. 2002, 113, 1-5.

(159) Giri, C.; Sahoo, P. K.; Puttreddy, R.; Rissanen, K.; Mal, P. Solvent-Free Ball-Milling Subcomponent Synthesis of Metallosupramolecular Complexes. Chem. - Eur. J. 2015, 21, 6390-6393.

(160) Tan, Y.-H.; Yang, L.-F.; Cao, M.-L.; Wu, J.-J.; Ye, B.-H. Liquid-Assisted Solid-State Reaction: Assembly Of $(6,3)$ and $(10,3)$ Hydrogen-Bonded Networks Based On $\left[\mathrm{M}(\mathrm{Hbiim})_{3}\right]$ By Oxidation Of $\left[\mathrm{M}\left(\mathrm{H}_{2} \mathrm{biim}\right)_{3}\right]^{2+}$ Complexes In The Presence Of Acetate Anions. CrystEngComm 2011, 13, 4512-4518.

(161) Dabirmanesh, Q.; Roberts, R. M. G. The Synthesis Of Iron Sandwich Complexes By Microwave Dielectric Heating Using A Simple Solid $\mathrm{CO}_{2}$-Cooled Apparatus In An Unmodified Commercial Microwave Oven. J. Organomet. Chem. 1993, 460, C28-C29.

(162) Dabirmanesh, Q.; Fernando, S. I. S.; Roberts, R. M. G. Synthesis And Decomplexation Of ( $\eta$-arene) $(\eta$-cyclopentadienyl)Iron(II) Hexafluorophosphates Using Microwave Dielectric Heating. J. Chem. Soc., Perkin Trans. 1 1995, 1, 743-749.

(163) Roberts, R. M. G. Synthesis of $\left(\eta^{6}\right.$-arene $)\left(\eta^{5}\right.$-cyclopentadienyl) Iron (II) Complexes With Heteroatom And Carbonyl Substituents Part II, Amino substituents. J. Organomet. Chem. 2006, 691, 49264930.

(164) Roberts, R. M. G. Synthesis of $\left(\eta^{6}\right.$-arene $)\left(\eta^{5}\right.$-cyclopentadienyl) iron (II) complexes With Heteroatom And Carbonyl Substituents. Part I: Oxygen and carbonyl substituents. J. Organomet. Chem. 2006, 691, 2641-2647.

(165) Garringer, S. M.; Hesse, A. J.; Magers, J. R.; Pugh, K. R.; O'Reilly, S. A.; Wilson, A. M. Microwave Synthesis of Benchmark Organo-Iron Complexes. Organometallics 2009, 28, 6841-6844.

(166) Pagnoux-Ozherelyeva, A.; Bolien, D.; Gaillard, S.; Peudru, F.; Lohier, J.-F.; Whitby, R. J.; Renaud, J.-L. Microwave Irradiation And Flow Chemistry For A Straightforward Synthesis Of Piano-Stool Iron Complexes. J. Organomet. Chem. 2014, 774, 35-42.

(167) Puciova, M.; Ertl, P.; Toma, S. Synthesis Of FerrocenylSubstituted Heterocycles: The Beneficial Effect Of The Microwave Irradiation. Collect. Czech. Chem. Commun. 1994, 59, 175-85.

(168) Villemin, D.; Martin, B.; Puciova, M.; Toma, S. Dry Synthesis Under Microwave Irradiation: Synthesis Of Ferrocenylenones. J. Organomet. Chem. 1994, 484, 27-31.

(169) Pedotti, S.; Patti, A. Microwave-Assisted Synthesis Of 1,5Dioxo-3-Substituted [5]Ferrocenophanes. J. Organomet. Chem. 2008, 693, 1375-1381.

(170) Kulikov, V. N.; Nikulin, R. S.; Arkhipov, D. E.; Rodionov, A. N.; Babusenko, E. S.; Kovalenko, L. V.; Belousov, Y. A. Ferrocenecarboxylic Acid And Microwave-Assisted Synthesis Of Ferrocenoyl Hydrazones. Russ. Chem. Bull. 2017, 66, 537-544.

(171) Jankova, S.; Cisarova, I.; Uhlik, F.; Stepnicka, P.; Kotora, M. Synthesis And Characterisation Of Dewar Benzene-Ferrocene Conjugates. Dalton Trans 2009, 3137-3139.

(172) Greene, D. L.; Mingos, D. M. P. Application Of Microwave Dielectric Loss Heating Effects For The Rapid And Convenient Synthesis Of Ruthenium(II) Polypyridine Complexes. Transition Met. Chem. 1991, 16, 71-72.

(173) Matsumura-Inoue, T.; Tanabe, M.; Minami, T.; Ohashi, T. A Remarkably Rapid Synthesis of Ruthenium(II) Polypyridine Complexes by Microwave Irradiation. Chem. Lett. 1994, 23, 2443-2446.

(174) Xiao, X.; Sakamoto, J.; Tanabe, M.; Yamazaki, S.; Yamabe, S.; Matsumura-Inoue, T. Microwave Synthesis And Electrospectrochemical Study On Ruthenium(II) Polypyridine Complexes. J. Electroanal. Chem. 2002, 527, 33-40.

(175) Bolink, H. J.; Cappelli, L.; Coronado, E.; Grätzel, M.; Nazeeruddin, M. K. Efficient and Stable Solid-State Light-Emitting Electrochemical Cell Using Tris(4,7-diphenyl-1,10-phenanthroline)ruthenium(II) Hexafluorophosphate. J. Am. Chem. Soc. 2006, 128, 46-47.
(176) Luis, E. T.; Ball, G. E.; Gilbert, A.; Iranmanesh, H.; Newdick, C. W.; Beves, J. E. Efficient Microwave-Assisted Synthesis And Characterization Of Key Ruthenium(II) Polypyridyl Complexes $\left[\mathrm{Ru}(\mathrm{bpy})_{3}\right]\left(\mathrm{PF}_{6}\right)_{2}, \quad\left[\mathrm{Ru}(\text { phen })_{3}\right]\left(\mathrm{PF}_{6}\right)_{2}, \quad\left[\mathrm{Ru}(\text { bpy })_{2}(\right.$ phen $\left.)\right]\left(\mathrm{PF}_{6}\right)_{2}$ and $\left[\mathrm{Ru}(\mathrm{phen})_{2}(\mathrm{bpy})\right]\left(\mathrm{PF}_{6}\right)_{2}$. J. Coord. Chem. 2016, 69, 1686-1694.

(177) Sun, Y.; Machala, M. L.; Castellano, F. N. Controlled Microwave Synthesis Of $\mathrm{Ru}(\mathrm{II})$ Synthons And Chromophores Relevant To Solar Energy Conversion. Inorg. Chim. Acta 2010, 363, 283-287.

(178) Wu, F.; Thummel, R. P. Ru(II) Complexes Of Crowded Delocalized Diimine Ligands. Inorg. Chim. Acta 2002, 327, 26-30.

(179) Sasaki, I.; Daran, J. C.; Ait-Haddou, H.; Balavoine, G. G. A. New Annelated 2,2':6',2"'-Terpyridines And Their Ru(II) Complexes: Synthesis And Characterisation. Inorg. Chem. Commun. 1998, 1, 354357.

(180) Beves, J. E.; Constable, E. C.; Housecroft, C. E.; Neuburger, M.; Schaffner, S.; Zampese, J. A. 4'-Chloro-2,2':6' $2^{\prime \prime}$-terpyridine (L): Ethyl Sulfate Salts Of $\left[\mathrm{H}_{2} \mathrm{~L}\right]^{2+}$ And The Single Crystal Structures Of $\left[\mathrm{H}_{2} \mathrm{~L}\right]\left[\mathrm{EtOSO}_{3}\right] \mathrm{Cl} \cdot \mathrm{H}_{2} \mathrm{O}$ and $\left[\mathrm{ML}_{2}\right]\left[\mathrm{PF}_{6}\right]_{2}$ with $\mathrm{M}=\mathrm{Fe}$ and $\mathrm{Ru}$. Inorg. Chem. Commun. 2008, 11, 1006-1008.

(181) Ziegler, M.; Monney, V.; Stoeckli-Evans, H.; Von Zelewsky, A.; Sasaki, I.; Dupic, G.; Daran, J.-C.; Balavoine, G. Complexes Of New Chiral Terpyridyl Ligands. Synthesis And Characterization Of Their Ruthenium(II) And Rhodium(III) Complexes. J. Chem. Soc., Dalton Trans. 1999, 667-676.

(182) Martineau, D.; Beley, M.; Gros, P. C. Pyrrolidine-Containing Polypyridines: New Ligands for Improved Visible Light Absorption by Ruthenium Complexes. J. Org. Chem. 2006, 71, 566-571.

(183) Rau, S.; Schäfer, B.; Grüßing, A.; Schebesta, S.; Lamm, K.; Vieth, J.; Görls, H.; Walther, D.; Rudolph, M.; Grummt, U. W.; Birkner, E. Efficient Synthesis Of Ruthenium Complexes Of The Type (R-bpy) ${ }_{2} \mathrm{RuCl}_{2}$ and $\left[(\mathrm{R}-\mathrm{bpy})_{2} \mathrm{Ru}(\mathrm{L}-\mathrm{L})\right] \mathrm{Cl}_{2}$ By MicrowaveActivated Reactions (R: H, Me, tert-But) (L-L: Substituted Bibenzimidazoles, Bipyrimidine, And Phenanthroline). Inorg. Chim. Acta 2004, 357, 4496-4503.

(184) Martineau, D.; Beley, M.; Gros, P. C.; Cazzanti, S.; Caramori, S.; Bignozzi, C. A. Tuning of Ruthenium Complex Properties Using Pyrrole- and Pyrrolidine-Containing Polypyridine Ligands. Inorg. Chem. 2007, 46, 2272-2277.

(185) Schwalbe, M.; Schäfer, B.; Görls, H.; Rau, S.; Tschierlei, S.; Schmitt, M.; Popp, J.; Vaughan, G.; Henry, W.; Vos, J. G. Synthesis and Characterisation of Poly(bipyridine)ruthenium Complexes as Building Blocks for Heterosupramolecular Arrays. Eur. J. Inorg. Chem. 2008, 2008, 3310-3319.

(186) Patil, S.; Deally, A.; Hackenberg, F.; Kaps, L.; Müller-Bunz, H.; Schobert, R.; Tacke, M. Novel Benzyl- or 4-CyanobenzylSubstituted $\mathrm{N}$-Heterocyclic (Bromo)(carbene)silver(I) and (Carbene)(chloro)gold(I) Complexes: Synthesis and Preliminary Cytotoxicity Studies. Helv. Chim. Acta 2011, 94, 1551-1562.

(187) Pezet, F.; Daran, J.-C.; Sasaki, I.; Ait-Haddou, H.; Balavoine, G. G. A. Highly Diastereoselective Preparation of Ruthenium Bis(diimine) Sulfoxide Complexes: New Concept in the Preparation of Optically Active Octahedral Ruthenium Complexes. Organometallics 2000, 19, 4008-4015.

(188) Abrahamsson, M.; Jäger, M.; Österman, T.; Eriksson, L.; Persson, P.; Becker, H.-C.; Johansson, O.; Hammarström, L. A $3.0 \mu$ s Room Temperature Excited State Lifetime of a Bistridentate $\mathrm{Ru}(\mathrm{II})-$ Polypyridine Complex for Rod-like Molecular Arrays. J. Am. Chem. Soc. 2006, 128, 12616-12617.

(189) Abrahamsson, M.; Jäger, M.; Kumar, R. J.; Österman, T.; Persson, P.; Becker, H.-C.; Johansson, O.; Hammarström, L. Bistridentate Ruthenium(II) polypyridyl-Type Complexes with Microsecond ${ }^{3}$ MLCT State Lifetimes: Sensitizers for Rod-Like Molecular Arrays. J. Am. Chem. Soc. 2008, 130, 15533-15542.

(190) Jäger, M.; Kumar, R. J.; Görls, H.; Bergquist, J.; Johansson, O. Facile Synthesis of Bistridentate RuII Complexes Based on 2,6Di(quinolin-8-yl)pyridyl Ligands: Sensitizers with Microsecond ${ }^{3}$ MLCT Excited State Lifetimes. Inorg. Chem. 2009, 48, 3228-3238. 
(191) Ott, C.; Wouters, D.; Thijs, H. M. L.; Schubert, U. S. New Preparation and Purification Methods for Metallo-Supramolecular Block Copolymers. J. Inorg. Organomet. Polym. Mater. 2007, 17, 241249.

(192) Funaki, T.; Yanagida, M.; Onozawa-Komatsuzaki, N.; Kasuga, K.; Kawanishi, Y.; Kurashige, M.; Sayama, K.; Sugihara, H. Synthesis Of A New Class Of Cyclometallated Ruthenium(II) Complexes And Their Application In Dye-Sensitized Solar Cells. Inorg. Chem. Commun. 2009, 12, 842-845.

(193) Grabulosa, A.; Beley, M.; Gros, P. C. Remarkable Effect of 4Substituted 2,2'-Bipyridine Ligands on the Stereochemistry of Ruthenium(II) Complexes. Eur. J. Inorg. Chem. 2008, 2008, 17471751.

(194) Glasson, C. R. K.; Meehan, G. V.; Clegg, J. K.; Lindoy, L. F.; Smith, J. A.; Keene, F. R.; Motti, C. Microwave Synthesis of a Rare $\left[\mathrm{Ru}_{2} \mathrm{~L}_{3}\right]^{4+}$ Triple Helicate and Its Interaction with DNA. Chem. - Eur. J. 2008, 14, 10535-10538.

(195) Pascu, G. I.; Hotze, A. C. G.; Sanchez-Cano, C.; Kariuki, B. M.; Hannon, M. J. Dinuclear Ruthenium(II) Triple-Stranded Helicates: Luminescent Supramolecular Cylinders That Bind and Coil DNA and Exhibit Activity against Cancer Cell Lines. Angew. Chem., Int. Ed. 2007, 46, 4374-4378.

(196) Munery, S.; Jaud, J.; Bonvoisin, J. Synthesis And Characterization Of Bis(Bipyridine)Ruthenium(II) Complexes With Bromo Or Protected Ethynyl $\beta$-Diketonato Ligands. Inorg. Chem. Commun. 2008, 11, 975-977.

(197) Jasimuddin, S.; Byabartta, P.; Mostafa, G.; Lu, T. H.; Sinha, C. Synthesis, Spectral Studies, Crystal Structure And Redox Properties Of Homoleptic Tris-Chelated Ruthenium(II)-Arylazoimidazoles. Polyhedron 2004, 23, 727-733.

(198) Herrero, S.; Jimenez-Aparicio, R.; Perles, J.; Priego, J. L.; Urbanos, F. A. First Microwave Synthesis Of Multiple Metal-Metal Bond Paddlewheel Compounds. Green Chem. 2010, 12, 965-967.

(199) Herrero, S.; Jimenez-Aparicio, R.; Perles, J.; Priego, J. L.; Saguar, S.; Urbanos, F. A. Microwave Methods For The Synthesis Of Paddlewheel Diruthenium Compounds With N,N-Donor Ligands. Green Chem. 2011, 13, 1885-1890.

(200) Baghurst, D. R.; Mingos, D. M. P. A New Reaction Vessel For Accelerated Syntheses Using Microwave Dielectric Super-Heating Effects. J. Chem. Soc., Dalton Trans. 1992, 1151-1155.

(201) Díaz-Valenzuela, M. B.; Phillips, S. D.; France, M. B.; Gunn, M. E.; Clarke, M. L. Enantioselective Hydrogenation and Transfer Hydrogenation of Bulky Ketones Catalysed by a Ruthenium Complex of a Chiral Tridentate Ligand. Chem. - Eur. J. 2009, 15, 1227-1232.

(202) Delgado-Martínez, P.; Elvira-Bravo, A.; González-Prieto, R.; Priego, L. J.; Jimenez-Aparicio, R.; Torres, R. M. Synthesis of $\mathrm{Ru}_{2} \mathrm{Br}\left(\mu-\mathrm{O}_{2} \mathrm{CC}_{6} \mathrm{H}_{4}-\mathrm{R}\right)_{4}(\mathrm{R}=o-\mathrm{Me}, m-\mathrm{Me}, p-\mathrm{Me})$ Using Microwave Activation: Structural and Magnetic Properties. Inorganics 2014, 2, 524-536.

(203) Cortijo, M.; Delgado-Martínez, P.; González-Prieto, R.; Herrero, S.; Jiménez-Aparicio, R.; Perles, J.; Priego, J. L.; Torres, M. R. Microwave And Solvothermal Methods For The Synthesis Of Nickel And Ruthenium Complexes With 9-Anthracene Carboxylate Ligand. Inorg. Chim. Acta 2015, 424, 176-185.

(204) Schmidlehner, M.; Kuhn, P.-S.; Hackl, C. M.; Roller, A.; Kandioller, W.; Keppler, B. K. Microwave-Assisted Synthesis Of NHeterocycle-Based Organometallics. J. Organomet. Chem. 2014, 772773, 93-99.

(205) Tönnemann, J.; Risse, J.; Grote, Z.; Scopelliti, R.; Severin, K. Efficient and Rapid Synthesis of Chlorido-Bridged Half-Sandwich Complexes of Ruthenium, Rhodium, and Iridium by Microwave Heating. Eur. J. Inorg. Chem. 2013, 2013, 4558-4562.

(206) Zagermann, J.; Molon, M.; Metzler-Nolte, N. MicrowaveAssisted Synthesis Of The Tp Sandwich Compound $\mathrm{TpRu}(P-\mathrm{Br}-$ $\left.\mathrm{C}_{6} \mathrm{H}_{4} \mathrm{Tp}\right)$ And Application Of Its Benzoic Acid Derivative $\mathrm{TpRu}(P$ $\left.\left(\mathrm{CO}_{2} \mathrm{H}\right)-\mathrm{C}_{6} \mathrm{H}_{4} \mathrm{Tp}\right)$ In The Covalent Labelling Of Biomolecules. Dalton Trans 2011, 40, 1011-1015.

(207) Kuhnert, N.; Danks, T. N. Microwave Accelerated Synthesis Of Cyclopentadienyl Bis-Phosphine Ruthenium(II) Thiolato Com- plexes Using Focused Microwave Irradiation. J. Chem. Res. 2002, 2002, 66-68

(208) Albrecht, C.; Gauthier, S.; Wolf, J.; Scopelliti, R.; Severin, K. Microwave-Assisted Organometallic Syntheses: Formation of Dinuclear $\left[(\right.$ Arene $\left.) \mathrm{Ru}(\mu-\mathrm{Cl})_{3} \mathrm{RuCl}\left(\mathrm{L}-\mathrm{L}^{\prime}\right)\right]$ Complexes ( $\mathrm{L}-\mathrm{L}^{\prime}$ : Chelate Ligands with $P$-, $N$-, or $S$-Donor Atoms) by Displacement of Arene $\pi$ Ligands. Eur. J. Inorg. Chem. 2009, 2009, 1003-1010.

(209) Cesari, C.; Sambri, L.; Zacchini, S.; Zanotti, V.; Mazzoni, R. Microwave-Assisted Synthesis of Functionalized Shvo-Type Complexes. Organometallics 2014, 33, 2814-2819.

(210) Tardiff, B. J.; Decken, A.; Sean McGrady, G. MicrowaveAssisted Synthesis of $\left[\mathrm{Os}_{2} \mathrm{Cl}_{3}\left(\mathrm{PEt}_{2} \mathrm{Ph}\right)_{6}\right] \mathrm{Cl}$, Featuring The First Reported X-Ray Crystal Structure. Inorg. Chem. Commun. 2008, 11, 44-46.

(211) Romanov, A. S.; Muratov, D. V.; Kudinov, A. R. High-Yield Synthesis Of Osmium Complexes $\left[\left(\eta^{6}-\mathrm{C}_{6} \mathrm{H}_{6}\right) \mathrm{OsCl}_{2}\right]_{2}$, [CpOs $\left(\eta^{6}-\right.$ $\left.\left.\mathrm{C}_{6} \mathrm{H}_{6}\right)\right]^{+}$and $\mathrm{CpOs}\left(\eta^{5}\right.$-cyclohexadienyl). J. Organomet. Chem. 2013, 724, 177-179.

(212) Suresh Babu, A. R.; Gavaskar, D.; Raghunathan, R. An Expedient Ultrasonic Assisted One-Pot Four Component Synthesis Of Novel Ferrocene Grafted Pyrrolidine Heterocycles Via [3 + 2]Cycloaddition Of Azomethine Ylides. J. Organomet. Chem. 2013, 745-746, 409-416.

(213) Cappelletti, L.; Vaghi, L.; Rinaldi, L.; Rotolo, L.; Palmisano, G.; Cravotto, G.; Penoni, A. One-Pot Sonochemical Synthesis Of Ferrocenyl Derivatives Via A Three-Component Reaction In Aqueous Media. Ultrason. Sonochem. 2015, 27, 30-36.

(214) Aranzaes, J. R.; Astruc, D. Simple Synthesis Of Organo-Iron Complexes From Iron-Sandwich Raw Materials Using Visible Light. Inorg. Chim. Acta 2008, 361, 1-4.

(215) Ornelas, C.; Ruiz, J.; Astruc, D. Visible-Light Photolysis Of $\left[\mathrm{FeCp}\left(\eta^{6}\right.\right.$-toluene $\left.)\right]\left[\mathrm{PF}_{6}\right]$ As A Clean, Convenient And General Route To Iron-Vinylidene And Iron-Acetylide Complexes. J. Organomet. Chem. 2009, 694, 1219-1222.

(216) Sierra, M. A.; Mancheño, M. J.; Vicente, R.; Gómez-Gallego, M. Synthesis Of Ferrocene-Substituted 2-Azetidinones. J. Org. Chem. 2001, 66, 8920-8925.

(217) Suen, H. F.; Wilson, S. W.; Pomerantz, M.; Walsh, J. L. Photosubstitution Reactions Of Terpyridine Complexes Of Ruthenium(II). Inorg. Chem. 1989, 28, 786-791.

(218) Matsubara, Y.; Konno, H.; Kobayashi, A.; Ishitani, O. Quantitative Photochemical Formation of $[\mathrm{Ru}(\mathrm{tpy})(\mathrm{bpy}) \mathrm{H}]^{+}$. Inorg. Chem. 2009, 48, 10138-10145.

(219) Padhi, S. K.; Tanaka, K. Photo- and Electrochemical Redox Behavior of Cyclometalated $\mathrm{Ru}(\mathrm{II})$ Complexes Having a 3Phenylbenzo[b][1,6]naphthyridine Ligand. Inorg. Chem. 2011, 50, 10718-10723.

(220) Cooke, J.; Berry, D. E.; Fawkes, K. L. Photochemical Synthesis and Ligand Exchange Reactions of $\mathrm{Ru}(\mathrm{CO})_{4}\left(\eta^{2}\right.$-alkene $)$ Compounds. J. Chem. Educ. 2007, 84, 115-118.

(221) Gill, T. P.; Mann, K. R. Photochemical Properties Of The Cyclopentadienyl( $\eta^{6}$-benzene)ruthenium(II) cation. The Synthesis And Reactions Of A Synthetically Useful Intermediate: The Cyclopentadienyltris(acetonitrile)ruthenium(II) Cation. Organometallics 1982, 1, 485-488.

(222) Gutierrez, A. C.; Jamison, T. F. Scalable and Robust Synthesis of $\mathrm{CpRu}(\mathrm{MeCN})_{3} \mathrm{PF}_{6}$ via Continuous Flow Photochemistry. J. Flow Chem. 2012, 1, 24-27.

(223) Gutierrez, A. C.; Jamison, T. F. Continuous Photochemical Generation of Catalytically Active $[\mathrm{CpRu}]^{+}$Complexes from $\mathrm{CpRu}\left(\eta^{6}-\mathrm{C}_{6} \mathrm{H}_{6}\right) \mathrm{PF}_{6}$. Org. Lett. 2011, 13, 6414-6417.

(224) Braga, D.; Maini, L.; Polito, M.; Grepioni, F. Unexpected Solid-Solid Reaction Upon Preparation Of $\mathrm{KBr}$ Pellets And Its Exploitation In Supramolecular Cation Complexation. Chem. Commun. 2002, 2302-2303.

(225) Ogawa, M.; Hashizume, T.; Kuroda, K.; Kato, C. Intercalation Of 2,2'-Bipyridine And Complex Formation In The Interlayer Space Of Montmorillonite By Solid-Solid Reactions. Inorg. Chem. 1991, 30, 584-585. 
(226) Batisai, E.; Lusi, M.; Jacobs, T.; Barbour, L. J. A Mechanochemically Synthesised Solid Solution Enables Engineering Of The Sorption Properties Of A Werner Clathrate. Chem. Commun. 2012, 48, 12171-12173.

(227) Ofodile, S. E.; Smith, N. O. Liquid Chromatographic Fractionation Of Isotopic p-Xylenes With Tetrakis(4methylpyridine)nickel(II) thiocyanate. Anal. Chem. 1981, 53, 904905.

(228) Ni, J.; Pan, Y.-Z.; Wei, Z.-R.; Cong, Q.; Zhang, D.; Wang, Y.G.; Liu, X.-Y. The Structures, Solvent-Free Synthesis And Interconversions of The Complexes $\left[\mathrm{MCl}_{2}(\mathrm{CEAP})_{2}\right]$ And Their Hydrogen-Bonded Salts $[\mathrm{CEAPH}]_{2}\left[\mathrm{MCl}_{4}\right]\{\mathrm{M}=\mathrm{Zn}$, Co and $\mathrm{Hg}$, CEAP $=4$-[N,N-bis (2-cyanoethyl)amino $]$ pyridine $\}$. Inorg. Chem. Commun. 2013, 30, 17-20.

(229) Chen, T.; Liang, B.; Xin, X. Studies on Solid-Solid Reactions between 4-Methylbenzenamine and $\mathrm{CuCl}_{2} \cdot 2 \mathrm{H}_{2} \mathrm{O}, \mathrm{CoCl}_{2} \cdot 6 \mathrm{H}_{2} \mathrm{O}$, and $\mathrm{NiCl}_{2} \cdot 6 \mathrm{H}_{2} \mathrm{O}$. J. Solid State Chem. 1997, 132, 291-293.

(230) Tigineh, G. T.; Liu, L. K. Studies on Mechanochemistry: Solid Coordination Compounds from Primary Aromatic Amines and Cobalt(II) Chloride Hexahydrate. J. Chin. Chem. Soc. 2014, 61, $1180-1187$.

(231) Gomes, C. S. B.; Gomes, P. T.; Duarte, M. T. $\alpha$-Diimine Transition-Metal Complexes: Mechanochemistry - A New Synthetic Approach. J. Organomet. Chem. 2014, 760, 101-107.

(232) Cincic, D.; Kaitner, B. Schiff Base Derived From 2-Hydroxy-1Naphthaldehyde And Liquid-Assisted Mechanochemical Synthesis Of Its Isostructural $\mathrm{Cu}$ (II) and $\mathrm{Co}(\mathrm{II})$ Complexes. CrystEngComm 2011, 13, 4351-4357.

(233) Hernandez, J. G.; Bolm, C. $\left[\mathrm{Cp} * \mathrm{RhCl}_{2}\right]_{2}$ : Mechanosynthesis And Applications In C-H Bond Functionalisations Under Ball-Milling Conditions. Chem. Commun. 2015, 51, 12582-12584.

(234) Schumacher, C.; Crawford, D. E.; Raguž, B.; Glaum, R.; James, S. L.; Bolm, C.; Hernández, J. G. Mechanochemical Dehydrocoupling Of Dimethylamine Borane And Hydrogenation Reactions Using Wilkinson's Catalyst. Chem. Commun. 2018, 54, 8355-8358.

(235) Mishra, A. P.; Purwar, H.; Jain, R. K.; Gupta, S. K. Microwave Synthesis, Spectral, Thermal And Antimicrobial Studies Of Some $\mathrm{Co}(\mathrm{II}), \mathrm{Ni}(\mathrm{II})$ and $\mathrm{Cu}$ (II) Complexes Containing 2-Aminothiazole Moiety. E-J. Chem. 2012, 9, 1655-1666.

(236) Ali, S.; Singh, V. Microwave Assisted Synthesis, Spectroscopic Characterization And Biological Screening Of Macrocyclic Metal(II) Complexes Of Malonic Acid Dihydrazide And 5-Chloroisatin. Der Pharma Chem. 2016, 8, 208-214.

(237) Aswathy, R.; Mohanan, K. Microwave Assisted Synthesis, Characterisation and Fluorescence Studies of some Transition Metal Complexes with a Luminol Derivative. J. Fluoresc. 2017, 27, 11711181.

(238) Saha, M.; Nasani, R.; Das, M.; Mobin, S. M.; Pathak, B.; Mukhopadhyay, S. The Effect Of Remote Substitution On The Formation Of Preferential Isomers Of Cobalt(III)-Tetrazolate Complexes By Microwave Assisted Cycloaddition. Inorg. Chem. Front. 2014, 1, 599-610.

(239) Taylor, C. J.; Motevalli, M.; Richards, C. J. Synthesis of Planar Chiral Cobalt Metallocenes by Microwave-Assisted Diastereoselective Complexation. Organometallics 2006, 25, 2899-2902.

(240) Harcourt, E. M.; Yonis, S. R.; Lynch, D. E.; Hamilton, D. G. Microwave-Assisted Synthesis of Cyclopentadienyl-Cobalt Sandwich Complexes from Diaryl Acetylenes. Organometallics 2008, 27, 16531656.

(241) Bertrand, G.; Tortech, L.; Fichou, D.; Malacria, M.; Aubert, C.; Gandon, V. An Improved Protocol for the Synthesis of $\left[\left(\eta^{4}-\right.\right.$ $\left.\left.\mathrm{C}_{4} \mathrm{R}_{4}\right) \mathrm{Co}\left(\eta^{5}-\mathrm{C}_{5} \mathrm{H}_{5}\right)\right]$ Complexes. Organometallics 2012, 31, 126-132. (242) Keaney, G. F.; Wood, J. L. Rhodium Perfluorobutyramide $\left(\mathrm{Rh}_{2}(\mathrm{Pfm})_{4}\right):$ A Synthetically Useful Catalyst For Olefin Aziridinations. Tetrahedron Lett. 2005, 46, 4031-4034.

(243) Amarante, D.; Cherian, C.; Emmel, C.; Chen, H.-Y.; Dayal, S.; Koshy, M.; Megehee, E. G. Improved Synthetic Routes To Rhodium
Bipyridine Complexes: Comparison Of Microwave vs. Conventional Synthesis. Inorg. Chim. Acta 2005, 358, 2231-2238.

(244) Dömötör, O.; Aicher, S.; Schmidlehner, M.; Novak, M. S.; Roller, A.; Jakupec, M. A.; Kandioller, W.; Hartinger, C. G.; Keppler, B. K.; Enyedy, E. A. Antitumor Pentamethylcyclopentadienyl Rhodium Complexes Of Maltol And Allomaltol: Synthesis, Solution Speciation And Bioactivity. J. Inorg. Biochem. 2014, 134, 57-65.

(245) Baghurst, D. R.; Michael, D.; Mingos, P.; Watson, M. J. Application Of Microwave Dielectric Loss Heating Effects For The Rapid And Convenient Synthesis Of Organometallic Compounds. J. Organomet. Chem. 1989, 368, C43-C45.

(246) Pruszyński, M.; Bilewicz, A.; Zalutsky, M. R. Preparation of $\mathrm{Rh}\left[16 \mathrm{aneS}_{4} \text {-diol }\right]^{211}$ At and $\operatorname{Ir}\left[16 \mathrm{aneS}_{4} \text {-diol }\right]^{211}$ At Complexes as Potential Precursors for Astatine Radiopharmaceuticals. Part I: Synthesis. Bioconjugate Chem. 2008, 19, 958-965.

(247) Buslaeva, T. M.; Kravchenko, V. V.; Kopylova, E. V.; Kashiricheva, I. I.; Alekseeva, O. A.; Kazantsev, S. S. Synthesis And Structure Of Iridium Complex With Thiocarbamide. Crystallogr. Rep. 2008, 53, 775-780.

(248) Yoshikawa, N.; Masuda, Y.; Matsumura-Inoue, T. Rapid Synthesis of New Emitting Ir(III) Polypyridine Complexes. Chem. Lett. 2000, 29, 1206-1207.

(249) Yoshikawa, N.; Sakamoto, J.; Matsumura-Inoue, T.; Takashima, H.; Tsukahara, K.; Kanehisa, N.; Kai, Y. Electrochemical And Phosphorescent Properties Of New Ir(III) Complexes Coordinated By Various Bipyridine Derivatives. Anal. Sci. 2004, 20, $711-716$.

(250) Matsumura-Inoue, T.; Yamamoto, Y.; Yoshikawa, N.; Terashima, M.; Yoshida, Y.; Fujii, A.; Yoshino, K. Microwave Synthesis Of Novel Ir(III) Complexes And Their Application To An Electroluminescence Device. Opt. Mater. 2004, 27, 187-191.

(251) Yoshikawa, N.; Matsumura-Inoue, T. Electrochemical And Phosphorescent Properties Of New Mixed-Ligand Ir(III) Complexes Coordinated With Both Terpyridine And Various Bipyridine Derivatives. Anal. Sci. 2003, 19, 761-765.

(252) Yoshikawa, N.; Yamabe, S.; Kanehisa, N.; Kai, Y.; Takashima, H.; Tsukahara, K. Synthesis, Characterization, and DFT Investigation of $\operatorname{Ir}$ (III) Tolylterpyridine Complexes. Eur. J. Inorg. Chem. 2007, 2007, $1911-1919$

(253) Konno, H.; Sasaki, Y. Selective One-pot Synthesis of Facial Tris-ortho-metalated Iridium(III) Complexes Using Microwave Irradiation. Chem. Lett. 2003, 32, 252-253.

(254) Saito, K.; et al. Microwave Synthesis of Iridium(III) Complexes: Synthesis of Highly Efficient Orange Emitters in Organic Light-Emitting Devices. Jpn. J. Appl. Phys. 2004, 43, 2733-2734.

(255) Hoyano, J. K.; Graham, W. A. G. Oxidative Addition Of The Carbon-Hydrogen Bonds Of Neopentane And Cyclohexane To A Photochemically Generated Iridium(I) Complex. J. Am. Chem. Soc. 1982, 104, 3723-3725.

(256) Mak, K. H. G.; Chan, P. K.; Fan, W. Y.; Ganguly, R.; Leong, W. K. Photochemical Reaction of $\mathrm{Cp} * \operatorname{Ir}(\mathrm{CO})_{2}$ with $\mathrm{C}_{6} \mathrm{~F}_{5} \mathrm{X}(\mathrm{X}=\mathrm{CN}$, F): Formation of Diiridium(II) Complexes. Organometallics 2013, 32, $1053-1059$

(257) Kantchev, E. A. B.; O’Brien, C. J.; Organ, M. G. Palladium Complexes of $\mathrm{N}$-Heterocyclic Carbenes as Catalysts for CrossCoupling Reactions-A Synthetic Chemist's Perspective. Angew. Chem., Int. Ed. 2007, 46, 2768-2813.

(258) Cincic, D.; Juribasic, M.; Babic, D.; Molcanov, K.; Sket, P.; Plavec, J.; Curic, M. New Insight Into Solid-State Molecular Dynamics: Mechanochemical Synthesis Of Azobenzene/Triphenylphosphine Palladacycles. Chem. Commun. 2011, 47, 11543-11545.

(259) Juribasic, M.; Uzarevic, K.; Gracin, D.; Curic, M. Mechanochemical C-H Bond Activation: Rapid And Regioselective Double Cyclopalladation Monitored By In Situ Raman Spectroscopy. Chem. Commun. 2014, 50, 10287-10290.

(260) Aleksanyan, D. V.; Churusova, S. G.; Aysin, R. R.; Klemenkova, Z. S.; Nelyubina, Y. V.; Kozlov, V. A. The First Example Of Mechanochemical Synthesis Of Organometallic Pincer Complexes. Inorg. Chem. Commun. 2017, 76, 33-35. 
(261) Kumar, R.; Pandey, K.; Mondal, D.; Balakrishna, S. Reaction of $\mathrm{PdCl}_{2}$ with Diphenylacetylene Revisited: Effect of Solvents and Ball Milling on Cyclization and Crystal Structures of Dimeric Cyclobutenylpalladium Chloride Complexes. ChemistrySelect 2018, 3, $1242-1247$

(262) Beillard, A.; Bantreil, X.; Métro, T.-X.; Martinez, J.; Lamaty, F. Mechanochemistry for Facilitated Access To N,N-Diaryl NHC Metal Complexes. New J. Chem. 2017, 41, 1057-1063.

(263) Adams, C. J.; Lusi, M.; Mutambi, E. M.; Orpen, A. G. TwoStep Mechanochemical Synthesis of Carbene Complexes of Palladium(II) and Platinum(II). Cryst. Growth Des. 2017, 17, $3151-3155$.

(264) Adams, C. J.; Lusi, M.; Mutambi, E. M.; Guy Orpen, A. TwoStep Solid-State Synthesis Of PEPPSI-Type Compounds. Chem. Commun. 2015, 51, 9632-9635.

(265) Adams, C. J.; Haddow, M. F.; Hughes, R. J. I.; Kurawa, M. A.; Orpen, A. G. Coordination Chemistry Of Platinum And Palladium In The Solid-State: Synthesis Of Imidazole And Pyrazole Complexes. Dalton Trans 2010, 39, 3714-3724.

(266) Do, J.-L.; Tan, D.; Friščíc, T. Oxidative Mechanochemistry: Direct, Room-Temperature, Solvent-Free Conversion of Palladium and Gold Metals into Soluble Salts and Coordination Complexes. Angew. Chem., Int. Ed. 2018, 57, 2667-2671.

(267) Balema, V. P.; Wiench, J. W.; Pruski, M.; Pecharsky, V. K. Solvent-Free Mechanochemical Synthesis Of Two Pt Complexes: cis$\left(\mathrm{Ph}_{3} \mathrm{P}\right)_{2} \mathrm{PtCl}_{2}$ and cis- $\left(\mathrm{Ph}_{3} \mathrm{P}\right)_{2} \mathrm{PtCO}_{3}$. Chem. Commun. 2002, 16061607.

(268) Tella, A. C.; Eke, U. B.; Owalude, S. O. Solvent-Free Mechanochemical Synthesis And X-Ray Studies Of $\mathrm{Cu}$ (II) And $\mathrm{Ni}$ (II) Complexes Of 5-(3,4,5-Trimethoxybenzyl)Pyrimidine-2,4Diamine (Trimethoprim) In A Ball-Mill. J. Saudi Chem. Soc. 2016, 20, S376-S381.

(269) Cindric, M.; Uzelac, M.; Cincic, D.; Halasz, I.; Pavlovic, G.; Hrenar, T.; Curic, M.; Kovacevic, D. Three Routes To Nickel(II) Salicylaldehyde 4-Phenyl And 4-Methylthiosemicarbazonato Complexes: Mechanochemical, Electrochemical And Conventional Approach. CrystEngComm 2012, 14, 3039-3045.

(270) Ferguson, M.; Giri, N.; Huang, X.; Apperley, D.; James, S. L. One-Pot Two-Step Mechanochemical Synthesis: Ligand And Complex Preparation Without Isolating Intermediates. Green Chem. 2014, 16, 1374-1382.

(271) Crawford, D.; Casaban, J.; Haydon, R.; Giri, N.; McNally, T.; James, S. L. Synthesis By Extrusion: Continuous, Large-Scale Preparation Of MOFs Using Little Or No Solvent. Chem. Sci. 2015, 6, 1645-1649.

(272) Mishra, A. P.; Sharma, N.; Jain, R. K. Microwave Synthesis, Spectral, Thermal And Antimicrobial Studies Of Some Ni(II) And $\mathrm{Cu}(\mathrm{II})$ Schiff Base Complexes. Open J. Synth. Theory Appl. 2013, 2, $56-62$.

(273) Jabeen, M.; Mehmood, K.; Khan, M. A.; Nasrullah, M.; Maqbool, T.; Jabeen, F.; Afzal, M. Comparative Study Of Microwave Assisted And Conventional Synthesis Of Furfuraldehyde Based Hydrazone Derivatives And Their Metal Complexes With Biological Evaluation. Asian J. Chem. 2017, 29, 431-436.

(274) Shoemaker, K. A.; Leadbeater, N. E. A Fast And Easy Approach To The Synthesis Of Zeise's Salt Using Microwave Heating. Inorg. Chem. Commun. 2009, 12, 341-342.

(275) Petruzzella, E.; Chirosca, C. V.; Heidenga, C. S.; Hoeschele, J. D. Microwave-Assisted Synthesis Of The Anticancer Drug Cisplatin, cis- $\left[\mathrm{Pt}\left(\mathrm{NH}_{3}\right)_{2} \mathrm{Cl}_{2}\right]$. Dalton Trans 2015, 44, 3384-3392.

(276) Carlsson, M.; Eliasson, B. One-Pot Synthesis of trans Monoor Diarylalkynyl Substituted Platinum(II) Compounds with Tertiary Phosphine or Phosphite Ligands. Organometallics 2006, 25, 55005502 .

(277) Januário-Charmier, M. A.; Kukushkin, V. Y.; Pombeiro, A. J. L. Microwave-Assisted [2 + 3] Cycloaddition Of Nitrones To Platinum(II) And -(IV) Bound Organonitriles. Dalton Trans 2003, 25402543.
(278) Desai, B.; Danks, T. N.; Wagner, G. Ligand Discrimination In The Reaction Of Nitrones With $\left[\mathrm{PtCl}_{2}(\mathrm{PhCN})_{2}\right]$. Selective Formation Of Mono-Oxadiazoline And Mixed Bis-Oxadiazoline Complexes Under Thermal And Microwave Conditions. Dalton Trans 2004, 166-171.

(279) Lasri, J.; Januário-Charmier, M. A.; Haukka, M.; Pombeiro, A. J. L. Stereospecific Synthesis of Polysubstituted E-Olefins by Reaction of Acyclic Nitrones with Free and Platinum(II) Coordinated Organonitriles. J. Org. Chem. 2007, 72, 750-755.

(280) Lasri, J.; Januário-Charmier, M. A.; Guedes da Silva, M. F. C.; Pombeiro, A. J. L. Direct Synthesis Of (Imine)Platinum(II) Complexes By Iminoacylation Of Ketoximes With Activated Organonitrile Ligands. Dalton Trans 2006, 5062-5067.

(281) Mukhopadhyay, S.; Lasri, J.; Guedes da Silva, M. F. C.; Januário-Charmier, M. A.; Pombeiro, A. J. L. trans-Bis[5-(4fluorophenyl)tetrazolato] bis(triphenylphosphine)platinum(II). Acta Crystallogr., Sect. E: Struct. Rep. Online 2007, 63, m2656.

(282) Mukhopadhyay, S.; Lasri, J.; Januário-Charmier, M. A.; Guedes da Silva, M. F. C.; Pombeiro, A. J. L. Microwave Synthesis Of Mono- And Bis-Tetrazolato Complexes Via 1,3-Dipolar Cycloaddition Of Organonitriles With Platinum(Ii)-Bound Azides. Dalton Trans 2007, 5297-5304.

(283) Smolenski, P.; Mukhopadhyay, S.; Guedes da Silva, M. F. C.; Januário-Charmier, M. A.; Pombeiro, A. J. L. New Water-Soluble Azido- And Derived Tetrazolato-Platinum(II) Complexes With PTA. Easy Metal-Mediated Synthesis And Isolation Of 5-Substituted Tetrazoles. Dalton Trans 2008, 6546-6555.

(284) Gabano, E.; Gama, S.; Mendes, F.; Fregonese, F.; Paulo, A.; Ravera, M. Application Of Microwave-Assisted Heating To The Synthesis Of $\mathrm{Pt}(\mathrm{II})$ Complexes. Inorg. Chim. Acta 2015, 437, 16-19.

(285) Castan, P.; Labiad, B.; Villemin, D.; Wimmer, F. L.; Wimmer, S. Solid-State Cyclometallation Of The 1-Methyl-2,4'-Bipyridinium Complexes Of Palladium(II) And Platinum(II). J. Organomet. Chem. 1994, 479, 153-157.

(286) Godbert, N.; Pugliese, T.; Aiello, I.; Bellusci, A.; Crispini, A.; Ghedini, M. Efficient, Ultrafast, Microwave-Assisted Syntheses of Cycloplatinated Complexes. Eur. J. Inorg. Chem. 2007, 2007, 51055111.

(287) Sharma, K.; Singh, R.; Fahmi, N.; Singh, R. V. Microwave Assisted Synthesis, Characterization And Biological Evaluation Of Palladium And Platinum Complexes With Azomethines. Spectrochim. Acta, Part A 2010, 75, 422-427.

(288) Winkelmann, O. H.; Navarro, O. Microwave-Assisted Synthesis of $\mathrm{N}$-Heterocyclic Carbene- Palladium(II) Complexes. Adv. Synth. Catal. 2010, 352, 212-214.

(289) Tu, T.; Malineni, J.; Dötz, K. H. A Novel Pyridine-Bridged Bis-benzimidazolylidene Pincer Palladium Complex: Synthesis and Catalytic Properties. Adv. Synth. Catal. 2008, 350, 1791-1795.

(290) Gosiewska, S.; Martinez Herreras, S.; Lutz, M.; Spek, A. L.; Havenith, R. W. A.; van Klink, G. P. M.; van Koten, G.; Gebbink, R. J. M. K. Synthesis, Structure, and Catalytic Performance of Diastereopure Five-Coordinated NCN-Pincer Palladium(II) Complexes Bearing Bulky Amino Acid Substituents. Organometallics 2008, 27, 2549-2559.

(291) Dean, M. L.; Schmink, J. R.; Leadbeater, N. E.; Bruckner, C. Microwave-Promoted Insertion Of Group 10 Metals Into Free Base Porphyrins And Chlorins: Scope And Limitations. Dalton Trans 2008, $1341-1345$.

(292) Hanifehpour, Y.; Morsali, A.; Soltani, B.; Mirtamizdoust, B.; Joo, S. W. Ultrasound-Assisted Fabrication Of A Novel Nickel(II)Bis-Pyrazolyl Borate Two-Nuclear Discrete Nano-Structured Coordination Compound. Ultrason. Sonochem. 2017, 34, 519-524.

(293) Rakers, V.; Cadinu, P.; Edel, J. B.; Vilar, R. Development Of Microfluidic Platforms For The Synthesis Of Metal Complexes And Evaluation Of Their DNA Affinity Using Online FRET Melting Assays. Chem. Sci. 2018, 9, 3459-3469.

(294) Lipshutz, B. H.; Yamamoto, Y. Introduction: Coinage Metals in Organic Synthesis. Chem. Rev. 2008, 108, 2793-2795. 
(295) Sun, J.; Dai, F.; Yuan, W.; Bi, W.; Zhao, X.; Sun, W.; Sun, D. Dimerization of a Metal Complex through Thermally Induced SingleCrystal-to-Single-Crystal Transformation or Mechanochemical Reaction. Angew. Chem., Int. Ed. 2011, 50, 7061-7064.

(296) Bowmaker, G. A.; Hanna, J. V.; Skelton, B. W.; White, A. H. Solvent-Assisted Solid-State Synthesis: Separating The Chemical From The Mechanical In Mechanochemical Synthesis. Chem. Commun. 2009, 2168-2170.

(297) Bowmaker, G. A.; Hanna, J. V.; Hart, R. D.; Healy, P. C.; King, S. P.; Marchetti, F.; Pettinari, C.; Skelton, B. W.; Tabacaru, A.; White, A. H. Mechanochemical And Solution Synthesis, X-Ray Structure And IR And ${ }^{31} \mathrm{P}$ Solid State NMR Spectroscopic Studies Of Copper(I) Thiocyanate Adducts With Bulky Monodentate Tertiary Phosphine Ligands. Dalton Trans 2012, 41, 7513-7525.

(298) Bowmaker, G. A.; Di Nicola, C.; Pettinari, C.; Skelton, B. W.; Somers, N.; White, A. H. Mechanochemical Synthesis In Copper(II) Halide/Pyridine Systems: Single Crystal X-Ray Diffraction And IR Spectroscopic Studies. Dalton Trans 2011, 40, 5102-5115.

(299) Bowmaker, G. A.; Hanna, J. V.; Hart, R. D.; Skelton, B. W.; White, A. H. The Role Of Short-Range Diffusion In Solvent-Assisted Mechanochemical Synthesis Of Metal Complexes. Dalton Trans 2008, 5290-5292.

(300) Guo, F.; Shao, H.-d.; Yang, Q.; Famulari, A.; Marti-Rujas, J. Mechanochemical Dehydrochlorination And Chelation Reaction In The Solid State: From A Molecular Salt To A Coordination Complex. CrystEngComm 2014, 16, 969-973.

(301) Braga, D.; Grepioni, F.; Maini, L.; Brescello, R.; Cotarca, L. Simple And Quantitative Mechanochemical Preparation Of The First Zinc And Copper Complexes Of The Neuroleptic Drug Gabapentin. CrystEngComm 2008, 10, 469-471.

(302) Ohara, H.; Kobayashi, A.; Kato, M. Simple Manual Grinding Synthesis of Highly Luminescent Mononuclear $\mathrm{Cu}(\mathrm{I})$-Iodide Complexes. Chem. Lett. 2014, 43, 1324-1326.

(303) Kobayashi, A.; Hasegawa, T.; Yoshida, M.; Kato, M. Environmentally Friendly Mechanochemical Syntheses and Conversions of Highly Luminescent $\mathrm{Cu}(\mathrm{I})$ Dinuclear Complexes. Inorg. Chem. 2016, 55, 1978-1985.

(304) Beillard, A.; Métro, T.-X.; Bantreil, X.; Martinez, J.; Lamaty, F. $\mathrm{Cu}(0), \mathrm{O}_{2}$ And Mechanical Forces: A Saving Combination For Efficient Production Of Cu-NHC Complexes. Chem. Sci. 2017, 8, 1086-1089.

(305) Fang, G.; Bi, X. Silver-Catalysed Reactions Of Alkynes: Recent Advances. Chem. Soc. Rev. 2015, 44, 8124-8173.

(306) Roland, S.; Jolivalt, C.; Cresteil, T.; Eloy, L.; Bouhours, P.; Hequet, A.; Mansuy, V.; Vanucci, C.; Paris, J.-M. Investigation of a Series of Silver- $\mathrm{N}$-Heterocyclic Carbenes as Antibacterial Agents: Activity, Synergistic Effects, and Cytotoxicity. Chem. - Eur. J. 2011, 17, $1442-1446$.

(307) Eloy, L.; Jarrousse, A.-S.; Teyssot, M.-L.; Gautier, A.; Morel, L.; Jolivalt, C.; Cresteil, T.; Roland, S. Anticancer Activity of Silver$\mathrm{N}$-Heterocyclic Carbene Complexes: Caspase-Independent Induction of Apoptosis via Mitochondrial Apoptosis-Inducing Factor (AIF). ChemMedChem 2012, 7, 805-814.

(308) Iqbal, M. A.; Umar, M. I.; Haque, R. A.; Khadeer Ahamed, M. B.; Asmawi, M. Z. B.; Majid, A. M. S. A. Macrophage And Colon Tumor Cells As Targets For A Binuclear Silver(I) N-Heterocyclic Carbene Complex, An Anti-Inflammatory And Apoptosis Mediator. J. Inorg. Biochem. 2015, 146, 1-13.

(309) Bowmaker, G. A.; Chaichit, N.; Pakawatchai, C.; Skelton, B. W.; White, A. H. Solvent-Assisted Mechanochemical Synthesis Of Metal Complexes. Dalton Trans 2008, 2926-2928.

(310) Bowmaker, G. A.; Pakawatchai, C.; Saithong, S.; Skelton, B. W.; White, A. H. Structural And Spectroscopic Studies Of Some Adducts Of Silver(I) Halides With Thiourea And N-Ethyl Substituted Thioureas. Dalton Trans 2010, 39, 4391-4404.

(311) Bowmaker, G. A.; Pakawatchai, C.; Saithong, S.; Skelton, B. W.; White, A. H. 1:1 Complexes Of Silver(I) Thiocyanate With (Substituted) Thiourea Ligands. Dalton Trans 2009, 2588-2598.
(312) Bowmaker, G. A.; Effendy; Hanna, J. V.; Healy, P. C.; King, S. P.; Pettinari, C.; Skelton, B. W.; White, A. H. Solution And Mechanochemical Syntheses, And Spectroscopic And Structural Studies In The Silver(I) (Bi-)Carbonate: Triphenylphosphine System. Dalton Trans 2011, 40, 7210-7218.

(313) Bowmaker, G. A.; Hanna, J. V.; Skelton, B. W.; White, A. H. Mechanochemical And Solution Synthesis, And Crystal Structures And IR And Solid-State (CPMAS) NMR Spectroscopy Of Some Bis(Triphenylphosphine)Silver(I) Mono- And Di-Hydrogencitrate Systems. Dalton Trans 2012, 41, 5409-5417.

(314) Beillard, A.; Golliard, E.; Gillet, V.; Bantreil, X.; Métro, T.-X.; Martinez, J.; Lamaty, F. Expedient Mechanosynthesis of N,N-Dialkyl Imidazoliums and Silver(I)-Carbene Complexes in a Ball-Mill. Chem. - Eur. J. 2015, 21, 17614-17617.

(315) Beillard, A.; Bantreil, X.; Métro, T.-X.; Martinez, J.; Lamaty, F. Unraveling The Synthesis Of Homoleptic $\left[\mathrm{Ag}(\mathrm{N}, \mathrm{N} \text {-Diaryl-NHC })_{2}\right] \mathrm{Y}$ $\left(\mathrm{Y}=\mathrm{BF}_{4}, \mathrm{PF}_{6}\right)$ Complexes By Ball-Milling. Dalton Trans 2016, 45, $17859-17866$.

(316) Egbert, J. D.; Slawin, A. M. Z.; Nolan, S. P. Synthesis of NHeterocyclic Carbene Gold Complexes Using Solution-Phase and Solid-State Protocols. Organometallics 2013, 32, 2271-2274.

(317) Do, J. L.; Tan, D.; Friščić, T. Oxidative Mechanochemistry: Direct, Room-Temperature, Solvent-Free Conversion of Palladium and Gold Metals into Soluble Salts and Coordination Complexes. Angew. Chem., Int. Ed. 2018, 57, 2667-2671.

(318) Jobbágy, C.; Baranyai, P.; Marsi, G.; Rácz, B.; Li, L.; Naumov, P.; Deák, A. Novel Gold(I) Diphosphine-Based Dimers With Aurophilicity Triggered Multistimuli Light-Emitting Properties. J. Mater. Chem. C 2016, 4, 10253-10264.

(319) Sharma, R. K.; Sharma, C.; Sidhwani, I. T. Solventless and One-Pot Synthesis of $\mathrm{Cu}(\mathrm{II})$ Phthalocyanine Complex: A Green Chemistry Experiment. J. Chem. Educ. 2011, 88, 86-87.

(320) Landers, B.; Navarro, O. Microwave-Assisted Synthesis of ( $N$ Heterocyclic carbene) $\mathrm{MCl}$ Complexes of Group 11 Metals. Eur. J. Inorg. Chem. 2012, 2012, 2980-2982.

(321) Lenker, H. K.; Gray, T. G.; Stockland, R. A. Rapid Synthesis Of Arylgold Compounds Using Dielectric Heating. Dalton Trans 2012, 41, 13274-13276.

(322) Shaw, A. P.; Tilset, M.; Heyn, R. H.; Jakobsen, S. Microwave Methods For The Synthesis Of Gold(III) Complexes. J. Coord. Chem. 2011, 64, 38-47.

(323) Hojaghani, S.; Hosaini Sadr, M.; Morsali, A. Sonochemical Synthesis Of Two New Copper(II) Complexes With Azo Ligands Derived From Anthranilic Acid And $\beta$-Naphtol. Ultrason. Sonochem. 2015, 26, 305-311.

(324) Catalano, V. J.; Malwitz, M. A. Short Metal-Metal Separations in a Highly Luminescent Trimetallic $\mathrm{Ag}(\mathrm{I})$ Complex Stabilized by Bridging NHC Ligands. Inorg. Chem. 2003, 42, 54835485.

(325) Opalka, S. M.; Park, J. K.; Longstreet, A. R.; McQuade, D. T. Continuous Synthesis and Use of $N$-Heterocyclic Carbene Copper(I) Complexes from Insoluble $\mathrm{Cu}_{2} \mathrm{O}$. Org. Lett. 2013, 15, 996-999.

(326) Chapman, M. R.; Shafi, Y. M.; Kapur, N.; Nguyen, B. N.; Willans, C. E. Electrochemical Flow-Reactor For Expedient Synthesis Of Copper- $\mathrm{N}$-Heterocyclic Carbene Complexes. Chem. Commun. 2015, 51, 1282-1284.

(327) Crawford, D. E.; Miskimmin, C. K.; Cahir, J.; James, S. L. Continuous Multi-Step Synthesis By Extrusion - Telescoping Solvent-Free Reactions For Greater Efficiency. Chem. Commun. 2017, 53, 13067-13070.

(328) Fernández-Bertrán, J. F.; Hernández, M. P.; Reguera, E.; YeeMadeira, H.; Rodriguez, J.; Paneque, A.; Llopiz, J. C. Characterization Of Mechanochemically Synthesized Imidazolates $\mathrm{Of}^{+1}, \mathrm{Zn}^{+2}, \mathrm{Cd}^{+2}$, and $\mathrm{Hg}^{+2}$ : Solid State Reactivity Of $\mathrm{nd}^{10}$ Cations. J. Phys. Chem. Solids 2006, 67, 1612-1617.

(329) Crawford, D. E.; James, S. L.; McNally, T. Use of Batch Mixing To Investigate the Continuous Solvent-Free Mechanical Synthesis of OLED Materials by Twin-Screw Extrusion (TSE). ACS Sustainable Chem. Eng. 2018, 6, 193-201. 
(330) Bagley, M. C.; Lin, Z.; Pope, S. J. A. Microwave-Assisted Synthesis And Complexation Of Luminescent Cyanobipyridyl-Zinc(II) Bis(Thiolate) Complexes With Intrinsic And Ancillary Photophysical Tunability. Dalton Trans 2010, 39, 3163-3166.

(331) Akabane, T.; Ohta, K.; Takizawa, T.; Matsuse, T.; Kimura, M. Discotic Liquid Crystals Of Transition Metal Complexes, 54: Rapid Microwave-Assisted Synthesis And Homeotropic Alignment Of Phthalocyanine-Based Liquid Crystals. J. Porphyrins Phthalocyanines 2017, 21, 476-492.

(332) Yadav, M.; Mishra, N.; Sharma, N.; Chandra, S.; Kumar, D. Microwave Assisted Synthesis, Characterization And Biocidal Activities Of Some New Chelates Of Carbazole Derived Schiff Bases Of Cadmium And Tin Metals. Spectrochim. Acta, Part A 2014, 132, 733-742.

(333) Paqhaleh, D. M. S.; Hashemi, L.; Amani, V.; Morsali, A.; Aminjanov, A. A. Synthesis Of Two New Nano-Structured Mercury(II) Complexes With 4-Methyl-4H-1,2,4-Triazole-3-Thiol Ligand By Sonochemical Method. Inorg. Chim. Acta 2013, 407, 1-6.

(334) Ma, X.; Lim, G. K.; Harris, K. D. M.; Apperley, D. C.; Horton, P. N.; Hursthouse, M. B.; James, S. L. Efficient, Scalable, and Solventfree Mechanochemical Synthesis of the OLED Material $\mathrm{Alq}_{3}(\mathrm{q}=8$ Hydroxyquinolinate). Cryst. Growth Des. 2012, 12, 5869-5872.

(335) Wang, J.; Ganguly, R.; Yongxin, L.; Diaz, J.; Soo, H. S.; Garcia, F. A Multi-Step Solvent-Free Mechanochemical Route To Indium(III) Complexes. Dalton Trans 2016, 45, 7941-7946.

(336) Wang, J.; Ganguly, R.; Yongxin, L.; Díaz, J.; Soo, H. S.; García, F. Synthesis and the Optical and Electrochemical Properties of Indium(III) Bis(arylimino)acenaphthene Complexes. Inorg. Chem. 2017, 56, 7811-7820.

(337) Prochowicz, D.; Franckevicius, M.; Cieslak, A. M.; Zakeeruddin, S. M.; Gratzel, M.; Lewinski, J. Mechanosynthesis Of The Hybrid Perovskite $\mathrm{CH}_{3} \mathrm{NH}_{3} \mathrm{PbI}_{3}$ : Characterization And The Corresponding Solar Cell Efficiency. J. Mater. Chem. A 2015, 3, 20772-20777.

(338) Nath, M.; Vats, M.; Roy, P. Design And Microwave-Assisted Synthesis Of Tri- And Dialkyltin(IV) Hippurates, Characterization, In Vitro Anti-Cancer And In Vivo Anti-Inflammatory Activities. Med. Chem. Res. 2015, 24, 51-62.

(339) Singh, R. V.; Chaudhary, P.; Chauhan, S.; Swami, M. Microwave-Assisted Synthesis, Characterization And Biological Activities Of Organotin (IV) Complexes With Some Thio Schiff Bases. Spectrochim. Acta, Part A 2009, 72, 260-268.

(340) Singh, R. V.; Chaudhary, P.; Poonia, K.; Chauhan, S. Microwave-Assisted Synthesis, Characterization And Biological Screening Of Nitrogen-Sulphur And Nitrogen-Oxygen Donor Ligands And Their Organotin(IV) Complexes. Spectrochim. Acta, Part A 2008, 70, 587-594.

(341) Dawara, L.; Singh, D.; Singh, R. V. Antimicrobial And Pesticidal Activity Of Some Organogermanium(IV) Complexes Synthesized Under Microwave Irradiation. Main Group Met. Chem. 2011, 34, 69-75.

(342) Shahrjerdi, A.; Hosseiny Davarani, S. S.; Najafi, E.; Amini, M. M. Sonoelectrochemical Synthesis Of A New Nano Lead(II) Complex With Quinoline-2-Carboxylic Acid Ligand: A Precursor To Produce Pure Phase Nano-Sized Lead(II) Oxide. Ultrason. Sonochem. 2015, 22, 382-390.

(343) Shahrjerdi, A.; Davarani, S. S. H.; Amini, M. M.; Najafi, E.; Janghouri, M.; Mohajerani, E.; Dehpour, A. Sonoelectrochemical Synthesis Of A Nanoscale Complex Of Lead(II) And 2-Methyl-8Hydroxyquinoline: Spectroscopic, Photoluminescence, Thermal Analysis Studies And Its Application In An OLED. J. Mater. Sci. 2014, 49, 441-449.

(344) Kesari, Y.; Athawale, A. Ultrasound Assisted Bulk Synthesis Of $\mathrm{CH}_{3} \mathrm{NH}_{3} \mathrm{PbI}_{3}$ Perovskite At Room Temperature. Mater. Lett. 2015, $159,87-89$.

(345) Bhooshan Kumar, V.; Gouda, L.; Porat, Z. e.; Gedanken, A. Sonochemical Synthesis of $\mathrm{CH}_{3} \mathrm{NH}_{3} \mathrm{PbI}_{3}$ Perovskite Ultrafine Nanocrystal Sensitizers For Solar Energy Applications. Ultrason. Sonochem. 2016, 32, 54-59.
(346) Rightmire, N. R.; Bruns, D. L.; Hanusa, T. P.; Brennessel, W. W. Mechanochemical Influence on the Stereoselectivity of Halide Metathesis: Synthesis of Group 15 Tris(allyl) Complexes. Organometallics 2016, 35, 1698-1706.

(347) Urano, M.; Wada, S.; Suzuki, H. A Novel Dry Route To Ortho-Functionalized Triarylbismuthanes That Are Difficult To Access By Conventional Wet Routes. Chem. Commun. 2003, 12021203.

(348) Woen, D. H.; Kotyk, C. M.; Mueller, T. J.; Ziller, J. W.; Evans, W. J. Tris(pentamethylcyclopentadienyl) Complexes of Late Lanthanides Tb, Dy, Ho, and Er: Solution and Mechanochemical Syntheses and Structural Comparisons. Organometallics 2017, 36, $4558-4563$.

(349) Kumari, B. S.; Rijulal, G.; Mohanan, K. Microwave Assisted Synthesis, Spectroscopic, Thermal and Biological Studies of Some Lanthanide(III) Chloride Complexes with a Heterocyclic Schiff Base. Synth. React. Inorg., Met.-Org., Nano-Met. Chem. 2009, 39, 24-30.

(350) Mohanan, K.; Kumari, B. S.; Rijulal, G. Microwave Assisted Synthesis, Spectroscopic, Thermal, And Antifungal Studies Of Some Lanthanide(III) Complexes With A Heterocyclic Bishydrazone. J. Rare Earths 2008, 26, 16-21.

(351) Nakashima, K.; Masuda, Y.; Matsumura-Inoue, T.; Kakihana, M. Microwave-Assisted Synthesis Of (Tris-Acetylacetonato)(2,9Dimethyl-4,7-Diphenyl-1,10-Phenanthroline)Terbium(III) Complex With Outstanding High Green Luminescence. J. Lumin. 2009, 129, 243-245.

(352) Wang, X. J.; Zhou, X. C. Microwave Synthesis And Characterization Of Europium Complexes With Cinnamic Acid And 2,2'-Bipyridine. Adv. Mater. Res. 2013, 670, 106-109.

(353) Zhou, X. C.; Zhong, L. P.; Lin, J. Y. Microwave Synthesis And Characterization Of Europium Complexes With Cinnamic Acid And Phenanthroline. Russ. J. Coord. Chem. 2009, 35, 460-463. 